\title{
A Search for Higgs Bosons in Final States with Multiple Tau Leptons at the DØ Experiment
}

A thesis submitted to the University of Manchester for the degree of

Doctor of Philosophy

in the Faculty of Engineering and Physical Sciences

September 2012

Louise Suter

School of Physics and Astronomy 


\section{Contents}

$\begin{array}{lr}\text { Abstract } & 20\end{array}$

$\begin{array}{ll}\text { Declaration } & 21\end{array}$

$\begin{array}{lr}\text { Copyright } & 22\end{array}$

1 Introduction $\quad 25$

2 Theoretical Overview 28

2.1 The Standard Model . . . . . . . . . . . . . . . . . . . . . . . 28

2.1 .1 Leptons . . . . . . . . . . . . . . . . . . . 30

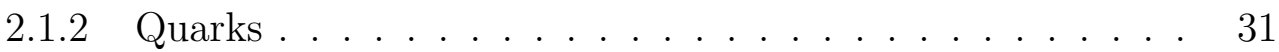

2.2 The mathematical formalism of the Standard Model . . . . . . . . 32

2.2.1 Spontaneous symmetry breaking and the Higgs boson . . . 33

2.2.2 Generating masses in the Standard Model . . . . . . . . . 35

2.3 Asymptotic freedom . . . . . . . . . . . . . . . . . 37

2.4 Motivation for beyond the Standard Model physics . . . . . . . . 38

2.5 The Supersymmetric Standard Model . . . . . . . . . . . . . . . . 39

2.5.1 NMSSM . . . . . . . . . . . . . . . . 39

2.6 Doubly charged Higgs bosons _. . . . . . . . . . . . . . . . . . 41

2.6.1 Left-Right symmetric models . . . . . . . . . . . . . . 42

2.6 .2 Little Higgs models . . . . . . . . . . . . . . . . . . . . . . 43 
2.7 Searches for Higgs bosons . . . . . . . . . . . . . . . . . 44

2.7.1 Standard Model Higgs bosons . . . . . . . . . . . . . 44

2.7.2 Doubly charged Higgs bosons . . . . . . . . . . . . 48

$3 \quad$ Experimental apparatus $\quad 53$

3.1 The Tevatron ......................... 53

3.1 .1 Proton Source . . . . . . . . . . . . . . . . . 54

3.1 .2 Linac . . . . . . . . . . . . . . . . . 54

3.1.3 Booster ........................... 55

3.1 .4 Main Injector . . . . . . . . . . . . . . . . . . . 55

3.1 .5 Anti-proton Source . . . . . . . . . . . . 56

3.1 .6 Recycler ..................... 56

3.1 .7 Tevatron Collider . . . . . . . . . . . . . 57

3.2 The $\mathrm{D} \varnothing$ detector . . . . . . . . . . . . . . . . . . 59

3.2.1 Central Tracking System . . . . . . . . . . . 61

3.2.2 Preshower Detectors . . . . . . . . . . . . . . . . . 64

3.2.3 Electromagnetic and Hadronic Calorimeters . . . . . . . 65

3.2.4 Muon System . . . . . . . . . . . . . . . . . . . 69

3.2.5 Luminosity Monitors . . . . . . . . . . . . . . . . 70

$\begin{array}{lll}4 & \text { Event reconstruction } & 71\end{array}$

4.1 Triggers ............................. 71

4.2 Detector component based reconstruction . . . . . . . . . . . . 73

4.2.1 Track reconstruction . . . . . . . . . . . . 74

4.2 .2 Vertex reconstruction . . . . . . . . . . . . . . 74

4.2.3 Calorimeter cell and cluster reconstruction . . . . . . . 75

4.3 Object reconstruction . . . . . . . . . . . . . . . . . 77

4.3 .1 Muon reconstruction . . . . . . . . . . . . . . 77 
4.3.2 Tau lepton reconstruction . . . . . . . . . . . . 81

4.3.3 Electron reconstruction . . . . . . . . . . . . 87

4.3 .4 Jet reconstruction . . . . . . . . . . . . . . . . . . . 90

4.3.5 Missing Transverse Energy . . . . . . . . . . . . . . . . . . 91

$\begin{array}{llr}5 & \text { Event simulation } & 93\end{array}$

5.1 Monte Carlo generators . . . . . . . . . . . . . . . 95

5.1 .1 PYтнIA ....................... 97

$5.1 .2 \quad$ AlPGEN . . . . . . . . . . . . . . . . . . . . . . 98

5.1 .3 Henwig $++\ldots \ldots \ldots$. . . . . . . . . . . . . . . 99

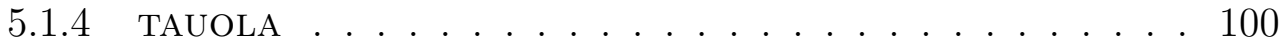

5.2 Detector simulation . . . . . . . . . . . . . 100

5.2 .1 D $\emptyset$ gstar . . . . . . . . . . . . . . . 100

5.2 .2 Zero bias overlay . . . . . . . . . . . . . . . 101

5.2.3 Trigger correction . . . . . . . . . . . . . . . . 102

5.2.4 Luminosity normalization . . . . . . . . . . . . . 102

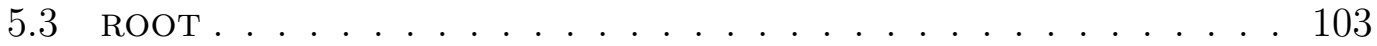

$6 \quad$ NMSSM in HERWIG $++\quad 104$

6.1 Implementation of the NMSSM . . . . . . . . . . . . . . . . . 104

6.2 Comparison to NMHDeCAY . . . . . . . . . . . . . . . . 107

6.3 Comparison of Herwig ++ at benchmark points . . . . . . . . . 109

6.4 The MSSM limit . . . . . . . . . . . . . . . . . . . 112

7 Analysis Methods $\quad 116$

7.1 Data samples . . . . . . . . . . . . . . . . . . 116

7.1 .1 Data skims . . . . . . . . . . . . 116

$7.1 .2 \quad$ Bad data . . . . . . . . . . . . . . . . . . 118

7.2 SingleMuonOR trigger . . . . . . . . . . . . . . . . 119 
7.3 Monte Carlo samples . . . . . . . . . . . . . . . . . . . . . 119

7.4 Monte Carlo correction factors . . . . . . . . . . . . . . . . 123

7.4.1 Beam position . . . . . . . . . . . . . 124

7.4.2 $W$ and $Z$ boson $p_{T}$ reweighting . . . . . . . . . . 124

7.4.3 Muon momentum resolution and scale . . . . . . . . 125

7.4.4 Muon ID and track reconstruction . . . . . . . . . . . 125

7.4.5 Tau lepton track identification . . . . . . . . . . . . . . 129

7.4.6 $N N_{\tau}$ efficiency . . . . . . . . . . . . . . 130

7.4.7 Tau lepton energy scale . . . . . . . . . . . . . 131

7.5 Selection requirements . . . . . . . . . . . . . . 133

7.5.1 Muon pre-selection . . . . . . . . . . . . . 134

7.5.2 Hadronically decaying tau lepton pre-selection . . . . . . . 135

7.6 Misreconstructed tau lepton events . . . . . . . . . . 136

$7.7 W+$ jets normalization $\ldots \ldots \ldots \ldots$

7.8 Instrumental background estimation . . . . . . . . . . . . . . 139

7.8.1 Instrumental background Method 1 . . . . . . . . . . . . 141

7.8.2 Instrumental background Method 2 . . . . . . . . . . 143

7.8.3 Application of instrumental background methods . . . . . 146

7.9 Multivariate Analysis Techniques . . . . . . . . . . . . . 147

7.9.1 Decision Trees . . . . . . . . . . . . . . . . . . . 148

7.9.2 Boosted Decision Trees . . . . . . . . . . . . . . . . 148

7.9 .3 MVA procedure . . . . . . . . . . . . . 150

7.10 Systematic Uncertainties . . . . . . . . . . . . . . . 153

7.10 .1 Cross section . . . . . . . . . . . . . . 153

7.10 .2 Luminosity . . . . . . . . . . . . . . . 154

7.10 .3 SingleMuonOR trigger . . . . . . . . . . . . . 154

7.10 .4 Muon Quality . . . . . . . . . . . . . . . . 154 
7.10 .5 Tau identification . . . . . . . . . . . . . . 155

7.11 Cross section limits . . . . . . . . . . . . . . 156

8 Doubly charged Higgs boson pair production 163

8.1 Data samples . . . . . . . . . . . . . . . . . 165

8.2 Monte Carlo samples . . . . . . . . . . . . . . . . . . 165

8.2.1 Background Monte Carlo samples . . . . . . . . . . . 165

8.2.2 Signal Monte Carlo samples . . . . . . . . . . . . . . . 166

8.3 Signal cross section normalization . . . . . . . . . . . 166

8.4 Selection requirements . . . . . . . . . . . . . . 167

8.4.1 Pre-selection requirements . . . . . . . . . . . . 167

8.4.2 Distributions at pre-selection . . . . . . . . . . 168

8.5 Instrumental background estimation . . . . . . . . . . . . . . . 172

8.5.1 Final selection requirements . . . . . . . . . . . . . 172

8.5.2 Distributions at final selection . . . . . . . . . . . . 173

8.6 Signal sample comparison . . . . . . . . . . . . . . . . . . . . 174

8.7 Results . . . . . . . . . . . . . . . . . . 174

8.7.1 Benchmark points for limiting setting . . . . . . . . . . 174

8.7.2 Summary of the $H^{++} H^{--} \rightarrow 4 \mu$ analysis . . . . . . . . . . 179

8.7.3 Final discriminants . . . . . . . . . . . . . . . 179

8.8 Systematic uncertainties . . . . . . . . . . . . . . . . . . 181

8.9 Cross section limits . . . . . . . . . . . . . . 185

9 SM Higgs bosons in the $\tau \tau \mu+X$ final states 194

9.1 Data samples . . . . . . . . . . . . . . . . . 195

9.2 Monte Carlo samples . . . . . . . . . . . . . . . . . . . . . . . 196

9.2.1 Background Monte Carlo samples . . . . . . . . . . . . . . 196

9.2.2 Signal Monte Carlo samples . . . . . . . . . . . . . . . . 196 
9.3 Inclusive trigger approach . . . . . . . . . . . . . . . . . 197

$9.4 \mathrm{~W}+$ jets normalization . . . . . . . . . . . . . 202

9.5 Instrumental background estimation . . . . . . . . . . . . . . 202

9.6 Selection requirements . . . . . . . . . . . . . . . 204

9.6.1 Pre-selection requirements . . . . . . . . . . 205

9.6.2 Muon and electron trilepton veto . . . . . . . . . 205

9.6.3 Distributions at pre-selection . . . . . . . . . 206

9.6.4 Final selection requirements . . . . . . . . . . 206

9.6.5 Distributions at final selection . . . . . . . . . . . 211

9.7 Signal sensitivity . . . . . . . . . . . . . . . . . . . 212

9.8 Multivariate analysis . . . . . . . . . . . . . . . 217

9.8 .1 BDT Pass $1 \ldots \ldots \ldots \ldots . \ldots \ldots$

9.8.2 BDT Pass 1 selection requirement . . . . . . . . . . 221

9.8 .3 BDT Pass $2 \ldots \ldots \ldots \ldots \ldots . \ldots \ldots . \ldots \ldots$

9.9 Systematic uncertainties . . . . . . . . . . . . . 226

9.10 Cross section limits . . . . . . . . . . . . . . . . 230

$\begin{array}{ll}10 \text { Conclusion } & 234\end{array}$

$\begin{array}{ll}\text { A BDT outputs } & 237\end{array}$

B The NMSSM Feynman Rules $\quad 242$

B.1 The Higgs boson-fermion vertices . . . . . . . . . . . . . . . . 242

B.2 Higgs boson-gauge bosons vertices . . . . . . . . . . . . . . . 243

B.2.1 Double Higgs boson-gauge boson vertices . . . . . . . . . 243

B.2.2 Higgs boson-gauginos vertices . . . . . . . . . . . . 244

B.2.3 Triple Higgs boson vertices . . . . . . . . . . . . . . 245

B.2.4 Scalar fermion-Higgs boson vertices . . . . . . . . . 246 
References

Total word count: 51,151 


\section{List of Tables}

4.1 The muon requirements for the different values of Nseg. . . . . . . 79

4.2 The decays of tau leptons which correspond to the three tau leptons

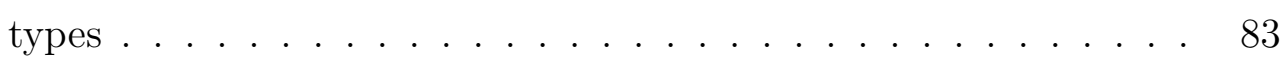

4.3 Quality requirements for the electrons quality definitions . . . . 90

6.1 NMHDECAY input parameters, used for the comparison of NMHDECAY to Herwig $++\ldots \ldots$. . . . . . . . . . . . . 109

6.2 Comparison of the partial widths of the heaviest Higgs boson, $h_{3}$, as calculated by NMHDECAY and Herwig $++\ldots$. . . . . . . . 110

6.3 Benchmark parameters for the five points used for validation of NMSSM . . . . . . . . . . . . . . . . . . 112

6.4 Comparison of the lightest Higgs boson decay widths, at the five benchmark points . . . . . . . . . . . . . . . 113

6.5 NMHDECAY input parameters to calculate the MSSM and NMSSM spectrum files, for comparison in the MSSM limit. . . . . . . . . . 114

6.6 Comparison of MSSM and the NMSSM in the MSSM limit, as calculated by HeRwiG $++\ldots$. . . . . . . . . . . . . . . . 115

7.1 The integrated luminosity recorded for the different Run periods

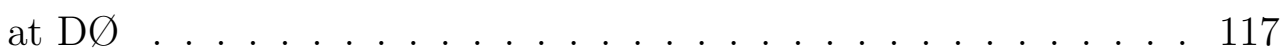

7.2 List of the single muon triggers f . . . . . . . . . . . . . . 119

7.3 The generated Monte Carlo samples for $Z$ boson processes . . . . 122 
7.4 The generated Monte Carlo samples for $W+$ jets, top anti-top quark, and diboson processes . . . . . . . . . . . . . . . . . 123

7.5 The k-factors for ALPGEN samples . . . . . . . . . . . . . . . . 124

7.6 Muon and tau lepton quality definition efficiencies per data epoch 130

7.7 The observed data compared to predicted MC events for the misreconstructed tau lepton study . . . . . . . . . . . . . . . . 138

7.8 The determined values of the $W+$ jets reweighting factor, $R_{W} \ldots 139$

7.9 The standard TMVA training parameters. . . . . . . . . . . 152

8.1 The signal MC samples generated for the $H^{ \pm \pm}$boson analysis . . 167

8.2 LO and NLO cross sections for the pair production of left handed and right handed $H^{ \pm \pm}$bosons . . . . . . . . . . . . . . . . . . . 168

8.3 Efficiency of the final selection requirements for the $H^{ \pm \pm}$boson analysis . . . . . . . . . . . . . . . . . . 174

8.4 The observed data and predicted background events for the $H^{ \pm \pm}$ boson analysis for both pre- and final selection . . . . . . . . . 178

8.5 Summary of the five benchmark points for the limit setting of the $H^{ \pm \pm}$boson analysis . . . . . . . . . . . . . . . . . . 179

8.6 Observed data and predicted background events for the $H^{ \pm \pm}$boson analysis for the four final discriminant channels . . . . . . . . . . 182

8.7 The determined systematic uncertainties for the $H^{ \pm \pm}$boson search 184

8.8 Expected and observed cross section limits for $\mathcal{B}\left(H^{ \pm \pm} \rightarrow \tau \tau\right)+$ $\mathcal{B}\left(H^{ \pm \pm} \rightarrow \mu \mu\right)=1$, per $H_{\mathrm{L}}^{ \pm \pm}$mass . . . . . . . . . . . . 189

8.9 Expected and observed cross section limits for $\mathcal{B}\left(H^{ \pm \pm} \rightarrow \tau \tau\right)+\mathcal{B}\left(H^{ \pm \pm} \rightarrow\right.$ $\mu \mu)=1$, per $H_{\mathrm{R}}^{ \pm \pm}$mass $\ldots \ldots \ldots \ldots \ldots$

8.10 Expected and observed cross section limits for $\mathcal{B}\left(H^{ \pm \pm} \rightarrow \mu \tau\right)=1 \quad 191$

8.11 Expected and observed cross sections for $H^{ \pm \pm}$with $\mathcal{B}\left(H^{++} \rightarrow \tau \tau\right)$ $=\mathcal{B}\left(H^{++} \rightarrow \mu \mu\right)=\mathcal{B}\left(H^{++} \rightarrow \mu \tau\right)=\frac{1}{3} \ldots \ldots \ldots . \ldots . \ldots 192$ 
8.12 Summary of expected and observed exclusion limits for the five benchmark points of the $H^{ \pm \pm}$boson analysis . . . . . . . . . . 193

9.1 MC simulation and data predictions for the SingleMuonOR and inclusive triggers . . . . . . . . . . . . . . . . 200

9.2 The multijet contribution for instrumental background Method 1 and 2 for the SM Higgs boson analysis . . . . . . . . . . 203

9.3 Prediction at pre-selection for the simulated MC, multijet backgrounds, data and the predicted signal for a associated production of a SM Higgs boson with $M_{H}=125 \mathrm{GeV} \ldots \ldots . . . . . . .210$

9.4 Prediction from backgrounds compared to data at both pre-selection and final selection, given per final selection requirement, for the SM Higgs boson analysis . . . . . . . . . . . . . . . . 213

9.5 Prediction from backgrounds compared to data at final selection, for all tau lepton types combined and separated into tau lepton types, for the SM Higgs boson analysis . . . . . . . . . . . . 213

9.6 The optimal signal and background rejection value for the BDT Pass 1, given per mass point . . . . . . . . . . . . . . . . 222

9.7 Prediction from MC compared to the observation in data after the selection requirement on the BDT Pass $1 \ldots$. . . . . . . . . 224

9.8 The $N N_{\tau}$ systematics per data epoch, for the $N N_{2012}$ and the selection requirements used, for the three tau lepton types . . . . 229

9.9 Systematic uncertainties in \% on the signal and background contributions, for the SM Higgs analysis . . . . . . . . . . . . . 230

9.10 Expected and observed cross section limits per SM Higgs boson mass, for BDT Pass $1 \ldots \ldots \ldots$. . . . . . . . . . . 231 
9.11 Expected and observed cross section limits as a ratio of the predicted SM Higgs cross section, per Higgs boson mass, for BDT Pass $2 \ldots \ldots \ldots \ldots \ldots \ldots \ldots$

9.12 Expected and observed cross section limits as a ratio of the predicted SM Higgs cross section, per Higgs boson mass, for the combined sample . . . . . . . . . . . . . . . . . . . 233 


\section{List of Figures}

2.1 The fermions and bosons in the Standard Model . . . . . . . . . . 29

2.2 A representation of the Higgs boson potential . . . . . . . . . . . 34

2.3 The predicted decays of the SM Higgs boson as a function of its

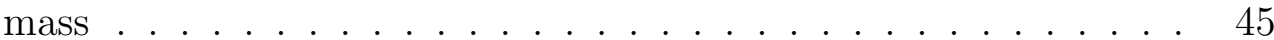

2.4 The SM Higgs boson production mechanisms at the LHC and Teva-

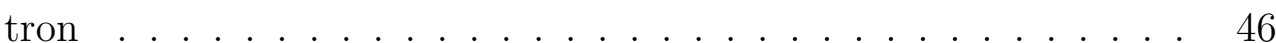

2.5 The Tevatron Higgs boson exclusion limits . . . . . . . . . . . . . 47

2.6 The best fit signal strength for the Tevatron Higgs boson searches, compared to SM predictions . . . . . . . . . . . . . . . . . . 48

2.7 The best fit signal strength for the LHC Higgs boson searches, compared to SM predictions . . . . . . . . . . . . . . . . . . 49

2.8 The LEP and Tevatron mass limits on the doubly charged Higgs boson ........................... 50

3.1 The Tevatron accelerator chain . . . . . . . . . . . . . 54

3.2 A typical store at $\mathrm{D} \varnothing \ldots \ldots \ldots \ldots$

3.3 Diagrammatic representation of the $\mathrm{D} \varnothing$ detector . . . . . . . . . . 60

3.4 A schematic of how particles will interact in the D $\varnothing$ detector . . . 61

3.5 The D $\varnothing$ inner tracking system . . . . . . . . . . . . . . . 63

3.6 Diagrammatic representation the SMT . . . . . . . . . . . . . 64 
3.7 Diagrammatic representation of both the solenoidal and toroidal magnetic fields, within the $\mathrm{D} \varnothing$ detector . . . . . . . . . . 65

3.8 The position of both the solenoidal and toroidal magnets, within the $\mathrm{D} \varnothing$ detector . . . . . . . . . . . . . . . 66

3.9 The layout of the $\mathrm{CC}$ and EC regions of the EM and hadronic calorimeters ..................... 67

3.10 A diagram of a calorimeter cell . . . . . . . . . . . . . 68

3.11 The layout of the cells within the calorimeters . . . . . . . . . . . 69

3.12 Diagrammatic representation of the PDT's and the scintillation counters of the muon system . . . . . . . . . . . . . 70

4.1 Diagrammatic representation of the triggering system . . . . . . . 72

4.2 The percentage of the decay of the tau leptons into hadrons and

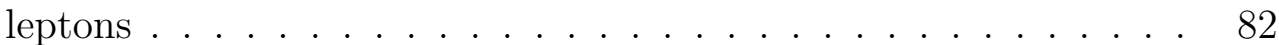

4.3 A comparison of the shape differences of the $N N_{2010}$ and $N N_{2012}$ $N N_{\tau} \mathrm{s} \ldots \ldots \ldots \ldots \ldots$

4.4 The efficiency compared to rejection of the $N N_{2012}$ compared to the $N N_{2010} \quad \ldots \ldots \ldots \ldots \ldots \ldots \ldots \ldots$

5.1 An example histogram, showing the data compared to the predicted contributions from different SM processes . . . . . . . . . . 94

5.2 An illustration of a MC simulated hard scatter process . . . . . . 96

5.3 An illustration of multiple interactions within the detector . . . . 97

7.1 Data and MC simulation comparison with and without the muon oversmearing . . . . . . . . . . . . . . . 126

7.2 The muon reconstruction efficiencies for the "Medium" quality re-

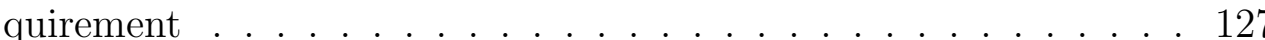


7.3 The muon reconstruction efficiencies the MediumNseg3 quality requirement . . . . . . . . . . . . . . . . . . . . 128

7.4 The track reconstruction efficiency for data and MC simulations . 129

7.5 The tau lepton $N N_{\tau}$ efficiency corrections and associated systematic uncertainty for $N N_{2010} \ldots \ldots \ldots \ldots$

7.6 The tau lepton $N N_{\tau}$ efficiency corrections and associated systematic uncertainty for $N N_{2012} \quad \ldots \ldots \ldots \ldots$

7.7 The ratio of $E_{T}^{c a l}$ to the $p_{T}^{\text {trk }}$ for determining the tau lepton energy scale correction . . . . . . . . . . . . . . . . . 134

7.8 Data distributions compared to the MC simulations for the misreconstructed tau leptons study . . . . . . . . . . . . . 137

7.9 Data distributions compared to $\mathrm{MC}$ simulations for the $W$-rich selection before and after the $W$-reweighting applied . . . . . . . . 140

7.10 Diagrammatic representation of instrumental background Method 1.142

7.11 The sum of the electric charge of the leptons at preselection . . . 144

7.12 Diagrammatic representation of instrumental background Method 2.145

7.13 A diagrammatic representation of the structure of a decision tree. 148

7.14 An example output of the BDT training . . . . . . . . . . 151

7.15 Efficiency distribution of the signal and background rejection as determined by TMVA . . . . . . . . . . . . . . . . . 152

7.16 LLR distribution as a function of the probability density . . . . . 161

7.17 The LLR distribution as a function of a model parameter . . . . . 162

8.1 A Feynman diagram showing the pair production of a $\mathrm{H}^{++} \mathrm{H}^{--}$ pair with $\mathcal{B}\left(H^{ \pm \pm} \rightarrow \mu \mu\right)=1 \ldots \ldots$. . . . . . . . . 164

8.2 The distribution of data and predicted backgrounds at pre-selection compared to the pair production of a $H_{\mathrm{L}}^{ \pm \pm}$boson, for the $p_{T}, \Delta R$ and $N$ of the leptons, and the $M_{T}$ distribution . . . . . . . 170 
8.3 The distribution of data and predicted backgrounds at pre-selection compared to the pair production of a $H_{\mathrm{L}}^{ \pm \pm}$boson, for the $\eta, \phi$ and $M$ of the leptons and the $E_{\mathrm{T}}$ distribution . . . . . . . . . . 17

8.4 The distribution of data and predicted background at final selection compared to the pair production of a $H_{\mathrm{L}}^{ \pm \pm}$boson, for the $p_{T}$, $\Delta R$ and $N$ of the leptons, and the $M_{T}$ distribution $\ldots \ldots . . .175$

8.5 The distribution of data and predicted background at final selection compared to the pair production of a $H_{\mathrm{L}}^{ \pm \pm}$boson, for the $\eta$, $\phi$ and $M$ of the leptons and the $\not_{\mathrm{T}}$ distribution . . . . . . 176

8.6 Comparison of left and right handed $H^{ \pm \pm}$bosons signal samples . 177

8.7 The discriminating variable for the $H^{++} H^{--} \rightarrow 4 \mu$ analysis. . . . 180

8.8 The discriminating variables for $H^{ \pm \pm}$analysis . . . . . . . . . . . 183

8.9 Exclusion regions of $H^{ \pm \pm}$mass for $\mathcal{B}\left(H^{ \pm \pm} \rightarrow \tau \tau\right)+\mathcal{B}\left(H^{ \pm \pm} \rightarrow \mu \mu\right)$ $=1 \ldots \ldots \ldots \ldots \ldots \ldots \ldots \ldots$

8.10 Cross section limits and LLR distributions for a left handed $H^{ \pm \pm}$ boson for, $\mathcal{B}\left(H^{ \pm \pm} \rightarrow \tau \tau\right)=1, \mathcal{B}\left(H^{ \pm \pm} \rightarrow \tau \tau\right)=\mathcal{B}\left(H^{ \pm \pm} \rightarrow \mu \mu\right)=$ 0.5 , and $\mathcal{B}\left(H^{ \pm \pm} \rightarrow \mu \mu\right)=1 \ldots \ldots \ldots \ldots$

8.11 Cross section limits and LLR distributions for a right handed $H^{ \pm \pm}$ boson for, $\mathcal{B}\left(H^{ \pm \pm} \rightarrow \tau \tau\right)=1, \mathcal{B}\left(H^{ \pm \pm} \rightarrow \tau \tau\right)=\mathcal{B}\left(H^{ \pm \pm} \rightarrow \mu \mu\right)=$ 0.5 , and $\mathcal{B}\left(H^{ \pm \pm} \rightarrow \mu \mu\right)=1 \ldots \ldots \ldots$. . . . . . . . . 188

8.12 Cross section limits and LLR distributions for a left and right handed for $\mathcal{B}\left(H^{ \pm \pm} \rightarrow \mu \tau\right)=1$ lepton pairs . . . . . . . . . . . . 191

8.13 Cross section limits for benchmark point (5) with $\mathcal{B}\left(H^{++} \rightarrow \tau \tau\right)$ $=\mathcal{B}\left(H^{++} \rightarrow \mu \mu\right)=\mathcal{B}\left(H^{++} \rightarrow \mu \tau\right)=\frac{1}{3} \ldots \ldots . \ldots . . \ldots 192$

9.1 A Feynman diagram showing the associated production of a SM Higgs boson, with a $W$ or $Z$ boson, where the Higgs boson decays into tau leptons . . . . . . . . . . . . . . . . . 195 
9.2 The product of the cross section and branching ratio in pb for the signal MC samples produced for the SM Higgs boson analysis . . 198

9.3 Data and generated MC distributions using the SingleMuonOr trigger and inclusive trigger f . . . . . . . . . . . . . . 199

9.4 The ratio of inclusive triggered events to SingleMuonOR triggered events. . . . . . . . . . . . . . . . . . 201

9.5 The distribution of data and predicted backgrounds at pre-selection compared to the associated production of a SM Higgs boson, for the $p_{T}, \Delta R$ and $N$ of the leptons and the $M_{T}$ distribution . . . 207

9.6 The distribution of data and predicted backgrounds at pre-selection compared to the associated production of a SM Higgs boson, for the $\eta, \phi$ and $M$ of the leptons and the $E_{\mathrm{T}}$ distribution $\ldots \ldots 208$

9.7 The distribution of observed data to predicted background at preselection compared to the associated production of a SM Higgs boson, shown per tau lepton type . . . . . . . . . . . 209

9.8 The distribution of data and predicted background at final selection compared to the associated production of a SM Higgs boson, for the $p_{T}, \Delta R$ and $N$ of the leptons, and the $M_{T}$ distribution . . 214

9.9 The distribution of data and predicted background at final selection compared to the associated production of a SM Higgs boson, for the $\eta, \phi$ and $M$ of the leptons and the $E_{\mathrm{T}}$ distribution . . . 215

9.10 The number of signal events selected at final selection from the different Higgs boson production modes at the Tevatron, as a function of the SM Higgs boson mass . . . . . . . . . . . . . . . . . . 216

9.11 The distributions of the selected signal events at final selection for WH Higgs boson signal . . . . . . . . . . . . . . . . . . . . . 216 
9.12 The distribution of data and predicted background at final selection compared to the associated production of a SM Higgs boson, for the $M\left(\mu, \tau_{1}\right)$ and the $p_{T}(\mathrm{H})$ distributions $\ldots \ldots \ldots$. . . . . 218

9.13 The signal versus background distributions for the nine variables used to train the BDT Pass $1 \ldots \ldots$. . . . . . . . . 220

9.14 Correlation matrices for both signal and background training sam-

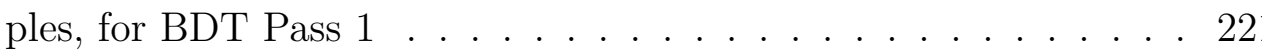

9.15 The signal versus background overtraining distribution for the BDT

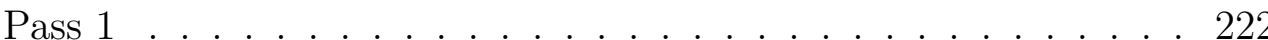

9.16 The BDT Pass 1 discriminant distribution, for a $125 \mathrm{GeV}$ Higgs

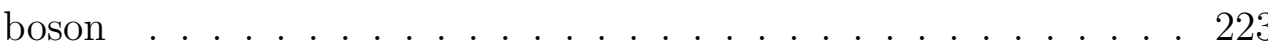

9.17 Comparison of the data with the predicted MC and data driven backgrounds for the input distributions for the BDT Pass 2, for $M_{H}=125 \mathrm{GeV}$ SM Higgs boson . . . . . . . . . . . . . 225

9.18 The separation between signal and background distributions for the four variables used to train the BDT Pass 2, for a $125 \mathrm{GeV}$ Higgs boson . . . . . . . . . . . . . . . . . 226

9.19 Correlation Matrices for both signal and background samples for the BDT Pass $2 \ldots \ldots \ldots \ldots \ldots$. . . . . . . . . . . . . 227

9.20 The overtraining distribution for the BDT Pass 2, for a SM Higgs boson mass of $125 \mathrm{GeV}$. . . . . . . . . . . . . . . . . . 227

9.21 The BDT Pass 2 final discriminant distribution for a $125 \mathrm{GeV}$ Higgs boson . . . . . . . . . . . . . . . . . . . . . . 228

9.22 The ratio of the expected and observed cross section limits to the SM Higgs boson cross section, as a function of the Higgs boson mass 232

A.1 The BDT Pass 1 discriminant distribution, 100 to $140 \mathrm{GeV}$. . . . 238

A.2 The BDT Pass 1 discriminant distribution, 145 to $200 \mathrm{GeV}$. . . . 239 
A.3 The BDT Pass 2 final discriminant distribution, 100 to $140 \mathrm{GeV}$. 240

A.4 The BDT Pass 2 final discriminant distribution, 145 to $200 \mathrm{GeV}$. 241 


\section{Abstract}

Name of the University: The University of Manchester

Candidate Name: Louise Suter

Degree Title: Doctor of Philosophy in the Faculty of Engineering and Physical Sciences

Thesis Title: A Search for Higgs Bosons in Final States with Multiple Tau Leptons at the DØ Experiment

Date: September 2012

Two searches for the production of Higgs bosons decaying into $\tau$ and $\mu$ leptons, using data collected with the $\mathrm{D} \varnothing$ detector at the Fermilab Tevatron $p \bar{p}$ collider, are presented. A search for the pair production of doubly charged Higgs bosons in the process $q \bar{q} \rightarrow H^{++} H^{--}$, where $H^{ \pm \pm}$decays to $\tau \tau, \mu \mu$ or $\tau \mu$ lepton pairs, with an integrated luminosity of up to $L=7.0 \mathrm{fb}^{-1}$, is presented. No significant excess of data over the expected SM background is observed and the results are used to set $95 \%$ C.L. limits on the pair production cross section of doubly charged Higgs bosons in the range $90<M_{H^{ \pm \pm}}<200 \mathrm{GeV}$. A second search for the production of the Standard Model Higgs boson in the final state $\tau \tau \mu+X$ is presented, using an integrated luminosity of $L=8.6 \mathrm{fb}^{-1}$. Again no significant excess of data is observed over the background expectation and 95\% C.L. limits are set on the observed cross section relative to the Standard Model prediction, in the range $100<M_{H}<200 \mathrm{GeV}$. 


\section{Declaration}

No portion of the work referred to in this thesis has been submitted in support of an application for another degree or qualification of this or any other university or other institution of learning.

Louise Suter

School of Physics and Astronomy

University of Manchester

Oxford Road

Manchester

M13 9PL

November 2012 


\section{Copyright}

Copyright in text of this thesis rests with the Author. Copies (by any process) either in full, or of extracts, may be made only in accordance with instructions given by the Author and lodged in the John Rylands University Library of Manchester. Details may be obtained from the Librarian. This page must form part of any such copies made. Further copies (by any process) of copies made in accordance with such instructions may not be made without the permission (in writing) of the Author.

The ownership of any intellectual property rights which may be described in this thesis is vested in The University of Manchester, subject to any prior agreement to the contrary, and may not be made available for use by third parties without the written permission of the University, which will prescribe the terms and conditions of any such agreement.

Further information on the conditions under which disclosures and exploitation may take place is available from the Head of the School of Physics and Astronomy. 


\section{Acknowledgments}

I would like to thank my supervisor Professor Stefan Söldner-Rembold for his continuing help and support throughout my thesis. I also extend my thanks to Professor Jeffrey Forshaw and Professor Peter Richardson for their help throughout my time spent working on the NMSSM and HeRIwIG++. For help and guidance throughout my $\mathrm{PhD}$, my thanks to go Timothy Head, Dr. Kostas Petridis and Dr. Maiko Takahashi. In addition I would like to thank my parents and sister for their motivation and belief in me throughout life.

My special thanks goes to Joseph Zennamo for always being there when I need him without ever being asked. 
"Stand back,

I'm going to try science"

$x k c d$ 


\section{Chapter 1}

\section{Introduction}

This thesis presents work that was performed at the DØ experiment and consists of two searches for Higgs bosons, a search for a doubly charged Higgs boson and a search for the Standard Model, SM, Higgs boson. It aims to explain the motivation for these searches and the tools necessary to perform them, from the experimental apparatus to the modeling software. It will explain in detail the methods used to perform a search using data recorded at $\mathrm{D} \varnothing$ and then cover the application of these tools and methods to the two specific cases. A project implementing the Next-to-Miminal-Supersymmetric Standard Model, NMSSM, will also be presented.

The relevant theories for the two searches, covering SM and beyond the Standard Model, BSM, phenomenology are described in Chapter 2. Both searches were performed at the DØ detector at the Tevatron collider, and details of the collider and the detector are given in Chapter 3, in Sections 3.1 and 3.2 respectively. The trigger system used by the $\mathrm{D} \varnothing$ experiment and the methods of object reconstruction are discussed in Chapter 4. The modeling, simulation and reconstruction software used in these analyses is covered in Chapter 5 and the project implementing the NMSSM, into the the HERwIG++ generator, in Chapter 6. An outline of the analysis methods used, along with the tools necessary to implement 
these methods are explained in Chapter 7, and the two analyses are covered in Chapter 8 and Chapter 9, respectively. Both searches were performed using Run II data, at a centre of mass energy of $\sqrt{s}=1.96 \mathrm{TeV}$ and required that there are two hadronically decaying tau leptons and one muon in the reconstructed final state.

The search for the pair production of doubly charged Higgs bosons, $q \bar{q} \rightarrow$ $H^{++} H^{--}$, decaying to $\tau \tau, \mu \mu$ or $\tau \mu$ lepton pairs, was performed using $7.0 \mathrm{fb}^{-1}$ of integrated luminosity and covered the following decay scenarios for the $\mathrm{H}^{++} \mathrm{H}^{--}$ boson pair:

1. $100 \%$ branching ratio into $\tau$ leptons, $\mathcal{B}\left(H^{ \pm \pm} \rightarrow \tau \tau\right)=1$.

2. $100 \%$ branching ratio into muons, $\mathcal{B}\left(H^{ \pm \pm} \rightarrow \mu \mu\right)=1$.

3. $100 \%$ branching ratio to tau muon lepton pairs, $\mathcal{B}\left(H^{ \pm \pm} \rightarrow \mu \tau\right)=1$.

4. $H^{++} H^{--}$boson pair decaying with equal branching ratio to $\tau \tau, \mu \mu$ and $\tau \mu$ lepton pairs, $\mathcal{B}\left(H^{ \pm \pm} \rightarrow \tau \tau\right)=\mathcal{B}\left(H^{ \pm \pm} \rightarrow \mu \mu\right)=\mathcal{B}\left(H^{ \pm \pm} \rightarrow \mu \tau\right)=\frac{1}{3}$.

5. A $H^{ \pm \pm}$with a mixed branching ratio to tau leptons and muons,

$$
\mathcal{B}\left(H^{ \pm \pm} \rightarrow \tau \tau\right)+\mathcal{B}\left(H^{ \pm \pm} \rightarrow \mu \mu\right)=1 .
$$

The results of the search are used to set 95\% Confidence Level (C.L.) upper limits on the above scenarios, for both left and right handed doubly charged Higgs bosons, except for Case 4 for which right handed states are not applicable. Cases 1, 4 and 5 had not been searched for previously at a hadron collider.

A search for a Standard Model Higgs boson was performed with $8.6 \mathrm{fb}^{-1}$ of integrated luminosity. This search investigated the $\tau \tau \mu+X$ final states and therefore is most sensitive to the associated Higgs boson production modes. At low masses this search is sensitive to Higgs boson decays into tau leptons and at high masses the decay of the Higgs boson into a pair of $W$ bosons. This analysis 
used multivariate analysis, MVA, techniques to set 95\% C.L. upper limits on the ratio of the observed cross section to the Standard Model cross section. This Higgs boson decay channel had not been searched for previously at D $\varnothing$. The limits determined in this analysis are used in the production of the combined DØ and Tevatron limits on the cross section of a Standard Model Higgs boson [1] [2]. 


\section{Chapter 2}

\section{Theoretical Overview}

This section will cover the theoretical motivation behind the searches performed, starting with an overview of the Standard Model, SM, the mathematical model that describes the known fundamental particles and how they interact. The theory and motivation for the Higgs boson, the particle hypothesized to impart mass to the other fundamental particles, will also be described, as well as the motivation to look for extensions to the SM. The production and decay modes, for both a SM and a doubly charged Higgs boson, will be discussed. Throughout this chapter, and through the rest of the thesis, it will be assumed that $c=\hbar=1$.

\subsection{The Standard Model}

Particle physics is the study of fundamental particles and their interactions. The model used to describe these interactions is the Standard Model and models all known fundamental particles, as listed in Figure 2.1. These particles are separated into two types, bosons and fermions, defined by the spin of the particles, with bosons carrying an integer spin quantum number and fermions a half integer spin quantum number. Fermions are further categorized into two types, quarks and leptons. All fundamental particles have a partner with the same mass but 
opposite charge, their anti-particle.

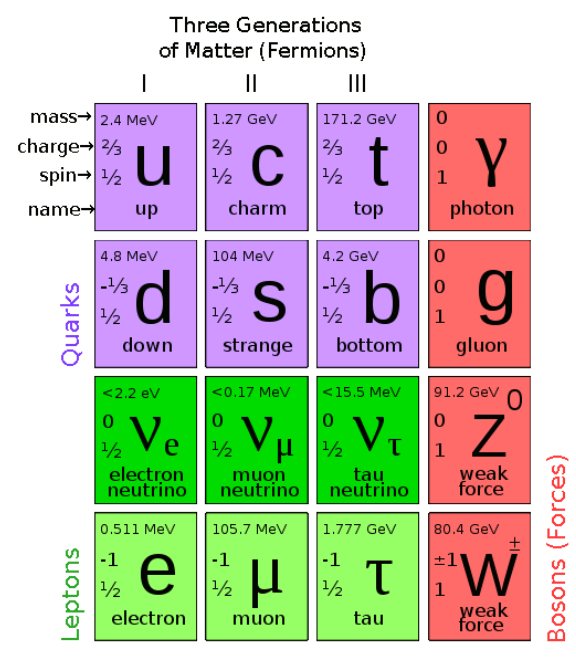

Figure 2.1: The fermions and bosons in the SM, showing the mass, electric charge and spin quantum number.

The interactions of the particles are governed by four forces, the electromagnetic, EM, force, the strong and weak nuclear forces, and gravity. At the scales and masses studied in particle physics, gravity has a negligible effect and therefore will not be discussed further. These forces each have an associated boson (or bosons) that mediate the interactions between the leptons, i.e. they are the force carriers of these interactions. For the weak nuclear force the associated bosons are the $W$ and $Z$ bosons and for the strong nuclear force they are the gluons, $g$. The photon, $\gamma$, is the boson associated with the EM interaction. Both the photon and the gluon are massless, meaning they are capable at interacting at large distances (though as discussed in Section 2.3, this picture is more complicated for the gluon), whereas the $W$ and $Z$ bosons are massive. The photon, gluon, and $Z$ boson all carry no electric charge whereas the $W$ boson carries an electric charge of \pm 1 in units of $e$, where $e$ is the electric charge of the electron. 


\subsubsection{Leptons}

There are three generations of leptons each corresponding to a specific flavour, each generation contains a charged lepton, (the electron, $e$, the $\mu$ lepton or muon, or the $\tau$ lepton or tauon) and a associated neutral lepton of the same flavour, a neutrino. The charged leptons all have a charge of $Q= \pm 1$, in units of $e$. The generations are seen to increase in mass with the $M_{e}=0.5 \mathrm{MeV}, M_{\mu}=105.7$ $\mathrm{MeV}$, and $M_{\tau}=1777 \mathrm{MeV}$. The generations are,

$$
\left(\begin{array}{c}
\nu_{e} \\
e^{-}
\end{array}\right),\left(\begin{array}{c}
\nu_{\mu} \\
\mu^{-}
\end{array}\right),\left(\begin{array}{c}
\nu_{\tau} \\
\tau^{-}
\end{array}\right)
$$

each with an associated set of anti-particles,

$$
\left(\begin{array}{c}
e^{+} \\
\overline{\nu_{e}}
\end{array}\right),\left(\begin{array}{c}
\mu^{+} \\
\overline{\nu_{\mu}}
\end{array}\right),\left(\begin{array}{l}
\tau^{+} \\
\overline{\nu_{\tau}}
\end{array}\right) .
$$

Each generation has an associated lepton number $L_{\ell}$ where $\ell=e, \mu$ or $\tau$, which are conserved in all know interactions, expect neutrino oscillations. The lepton number is defined by

$$
L_{\ell}=N\left(\ell^{-}\right)-N\left(\ell^{+}\right) .
$$

where $N\left(\ell^{-}\right)$is number of leptons of lepton flavour $\ell$, and $N\left(\ell^{+}\right)$the number of anti-leptons of lepton flavour $\ell$. Leptons can interact both through the EM force and the weak nuclear force, but as they are not a carrier of colour, the quantum number associated with the strong force, they do not interact through the strong nuclear force. To conserve charge and lepton number, the allowed leptonic decays of the $W$ and $Z$ bosons are: $W^{+} \rightarrow \ell^{+} \nu_{\ell}, W^{-} \rightarrow \ell^{-} \overline{\nu_{\ell}}$ and $Z \rightarrow \ell^{+} \ell^{-}, Z \rightarrow \nu_{\ell} \overline{\nu_{\ell}}$. Searches have been performed for evidence of lepton number violation, as is predicted by some extensions to the SM (see Section 2.7.2), but as yet no evidence has been seen. 
The neutrino only interacts through the weak nuclear force and experimental limits on the possible upper values of their masses of $M_{\nu_{e}}<2.2 \mathrm{eV}, M_{\nu_{\mu}}<170$ $\mathrm{keV}$, and $M_{\nu_{\tau}}<15.5 \mathrm{MeV}$, have confirmed them to be light. These properties result in neutrinos having very low interaction rates, traveling through an average particle detector without a trace.

Electrons are stable particles but they quickly lose energy through radiation. The rate of this energy loss is proportional to the mass of the particles, resulting in it being thousands of times smaller for muons. This comparatively slow energy loss for a muon results in a very penetrating particle, generally referred to as a minimally ionizing particle. Both the muon and the tau lepton are unstable particles with lifetimes of $\tau_{\mu}=2.2 \times 10^{-6} \mathrm{~s}$ and $\tau_{\tau}=2.9 \times 10^{-13} \mathrm{~s}$, respectively.

\subsubsection{Quarks}

The second set of fermions described by the SM are quarks, which interact through the EM force, and through the weak and strong nuclear forces. There are no free quarks in nature and they are only seen in bound states, as hadrons. Combinations of three quarks are called baryons and combinations of two quarks mesons. As for the leptons, there are six types of quarks which are categorized into three generations, with each generation increasing in mass:

$$
\left(\begin{array}{l}
u \\
d
\end{array}\right),\left(\begin{array}{l}
c \\
s
\end{array}\right),\left(\begin{array}{l}
t \\
b
\end{array}\right)
$$

and their corresponding anti-particles

$$
\left(\begin{array}{l}
\bar{d} \\
\bar{u}
\end{array}\right),\left(\begin{array}{l}
\bar{s} \\
\bar{c}
\end{array}\right),\left(\begin{array}{l}
\bar{b} \\
\bar{t}
\end{array}\right) .
$$

The quarks are given the following names, down quark, $d$, up quark, $u$, strange quark, $s$, charm quark, $c$, bottom quark, $b$, and top quark, $t$. The $u, c$, and $t$ quarks carry an electric charge of $2 / 3$ and the $d, s$, and $b$ quarks and an electric 
charge of $-1 / 3$. The number of baryons is conserved in the electromagnetic and the strong nuclear interactions and violated in weak nuclear interactions. This is quantified through the conservation of the baryon number, $B$, defined as,

$$
B=\frac{1}{3}[N(q)-N(\bar{q})]
$$

where $N(q)$ is the total number of quarks present and $N(\bar{q})$ is the total number of anti-quarks present. Over 200 hadrons have so far been identified, the most common baryons being the proton $(u u d)$ and neutron $(u d d)$. The lightest and hence most commonly produced meson is the $\pi$ meson, or pion, which is a bound state of a $u$ and $d$ quark. There are three types of pions, $\pi^{+}=u \bar{d}, \pi^{-}=d \bar{u}$, and $\pi^{0}=\frac{1}{\sqrt{2}}(u \bar{u}-d \bar{d})$.

\subsection{The mathematical formalism of the Stan- dard Model}

The gauge group of the $\mathrm{SM}$ is $\mathrm{U}(1) \otimes \mathrm{SU}(2) \otimes \mathrm{SU}(3)$. $\mathrm{U}(1)$ is an Abelian group

of quantum number hypercharge, Y, coupling constant, $g^{\prime}$, and gauge boson, $B_{\mu}$. $\mathrm{SU}(2)$ is a non-Abelian group described by the coupling constant $g$ and gauge bosons $W_{\mu}^{1}, W_{\mu}^{2}$, and $W_{\mu}^{3}$. These two groups, $\mathrm{U}(1) \otimes \mathrm{SU}(2)$, describe the electroweak sector. For the physical electroweak bosons, the $\gamma$ and $Z$ bosons, are superpositions of the $\mathrm{U}(1)$ and $\mathrm{SU}(2)$ gauge bosons, whereas the $W$ boson is a superposition of only $\mathrm{SU}(2)$ states. $\mathrm{SU}(3)$ is the non-Abelian group describing the strong nuclear interaction, with the coupling constant, $g_{s}$ and eight associated bosons, the gluons.

The SM Lagrangian can be split into several terms which describe the bosons and fermions and their interactions, 


$$
\mathcal{L}_{\mathrm{SM}}=\mathcal{L}_{\text {gauge bosons kinetic }}+\mathcal{L}_{\text {fermion kinetic }}+\mathcal{L}_{\text {fermion masses }}+\mathcal{L}_{\text {Higgs boson }}
$$

for simplicity the kinetic terms will be ignored here and only the terms necessary for the generation of mass will be considered.

\subsubsection{Spontaneous symmetry breaking and the Higgs bo- son}

To explain how the Higgs mechanism generates mass terms for the SM particles the concept of spontaneous symmetry breaking must first be introduced. It allows the Lagrangian of the SM to keep its local invariance in its lowest energy state, but for the ground state not to be locally invariant, i.e. it has a non zero vacuum expectation value, VEV. This can be pictured, as shown in Figure 2.2, for a point mass with a potential, $V(\Phi)$, defined as,

$$
V(r)=-\mu^{2} \Phi \cdot \Phi+\lambda \Phi \cdot \Phi^{2}
$$

When a particle is at $\phi=0$, it is in a symmetric state but not the ground state. To reach the ground state the particle must pick a direction to go down the slope and in doing so it will break its symmetry.

To show how this results in mass terms for the gauge bosons, this picture can be extended to a U(1) Abelian gauge theory, for which the Lagrangian can be written as [4],

$$
\mathcal{L}=\left(D_{\mu} \Phi\right)^{*}\left(D^{\mu} \Phi\right)-\frac{1}{4} F_{\mu \nu} F^{\nu \mu}-V(\Phi)
$$

where $D_{\mu}$ is the covariant derivative, $F_{\mu \nu}$ is the field strength tensor. An additional potential, $V(\Phi)$, to allow for symmetry breaking has been included. $V(\Phi)$ is of the form, 


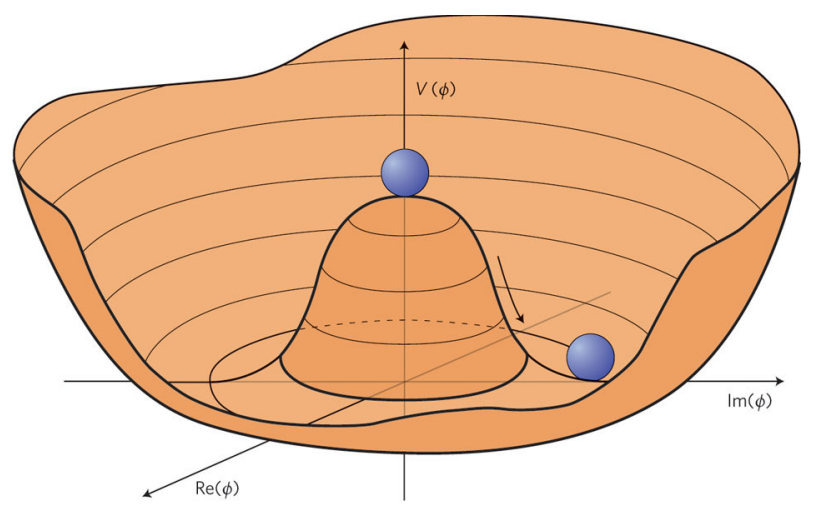

Figure 2.2: A representation of the Higgs boson potential [3].

$$
V(\Phi)=-\mu^{2} \Phi^{*} \Phi+\lambda\left|\Phi^{*} \Phi\right|^{2}
$$

The minimum of this potential will be at $\Phi=e^{i \theta} \sqrt{\frac{\mu^{2}}{2 \lambda}}=e^{i \theta} \frac{v}{\sqrt{2}}$, defining $v=\mu / \sqrt{\lambda}$. When setting $\theta$ to a specific value, the symmetry is broken and the ground state (the gauge invariant state) will no longer be zero. For convenience it is defined that $\theta=0$ therefore the VEV for this state will be $\langle\Phi\rangle=\frac{v}{\sqrt{2}}$.

To show that there is a particle associated with this potential, $\Phi$ can be expanded around its VEV [4]

$$
\Phi=\frac{e^{i \theta / v}}{\sqrt{2}}\left(\frac{\mu}{\sqrt{\lambda}}+H\right) \approx \frac{1}{\sqrt{2}}\left(\frac{\mu}{\sqrt{\lambda}}+H+i \phi\right) .
$$

Inserting this form of $\Phi$ back into the potential (Equation 2.10), it can be seen that terms of the form $\mu^{2} H^{2}$ are generated, i.e. mass terms for a Higgs field, $\mathrm{H}$, with a mass of $M_{H}=\sqrt{2} \mu$. In a similar way inserting this form of $\Phi$ into the Higgs interaction term of the Lagrangian, mass terms are generated for the gauge bosons [4]. 


\subsubsection{Generating masses in the Standard Model}

In this section it will be shown how masses can be generated for the SM particles by spontaneously breaking the $\mathrm{U}(1) \otimes \mathrm{SU}(2)$ gauge symmetry of the $\mathrm{SM}$, through the introduction of a Higgs doublet.

In the SM there are both left and right handed fermions, with the weak force only interacting with left handed particles. Left handed particles as defined with their momentum and spin vectors anti-aligned and right handed with them aligned. A Dirac field can be written as the sum of a left handed and a right handed particle,

$$
\psi=\psi_{L}+\psi_{R}
$$

where the left and right handed states can be written as [4]:

$$
\begin{aligned}
& \psi_{L}=P_{L} \psi \quad \text { with } \quad P_{L}=\frac{1-\gamma_{5}}{2} \\
& \psi_{R}=P_{R} \psi \quad \text { with } \quad P_{R}=\frac{1+\gamma_{5}}{2}
\end{aligned}
$$

$P_{L}$ and $P_{R}$ are projection operators, $P_{L} P_{L}=P_{L}, P_{R} P_{R}=P_{R}, P_{R} P_{L}=P_{L} P_{R}=0$, and $P_{L}+P_{R}=\mathbf{I}$ and $\gamma_{5}$ is the product of the Dirac matrices, $\gamma_{5}=i \gamma_{0} \gamma_{1} \gamma_{2} \gamma_{3} \gamma_{4}$.

For simplicity this description will be limited to one family of fermions, but the theory can be simply extended for the extra two. As only the left handed particles have been observed to couple to $W$ bosons, left and right handed particles are therefore assigned to different multiplets of $\mathrm{SU}(2)$, with the right handed states constructed as singlets. One generation of fermions can therefore be written as,

$$
q_{L}=\left(\begin{array}{c}
u_{L} \\
d_{L}
\end{array}\right), \quad u_{R}, \quad d_{R}, \quad \ell=\left(\begin{array}{c}
\nu_{L} \\
e_{L}
\end{array}\right), \quad e_{R}
$$

with the following assigned hypercharges $\mathrm{Y}(\ell)=-1 / 2, \mathrm{Y}\left(e_{R}\right)=-1, \mathrm{Y}\left(q_{L}\right)=1 / 6$ and $\mathrm{Y}\left(u_{R}\right)=2 / 3, \mathrm{Y}\left(d_{R}\right)=-1 / 3$, to reflect the observed charges of the particles. 
In the SM, explicit mass terms for the fermions create a problem as they would mix the left and right handed states which are in different multiplets of $\mathrm{SU}(2)$ [4]. To create mass terms for the fermions a gauge invariant interaction, a Yukawa interaction, can be constructed,

$$
\mathcal{L}_{\text {Yukawa }}=-h_{e} \bar{l}_{L}^{i} \Phi_{i} e_{R}+\text { h.c. }
$$

where h.c. is the hermitian conjugate and $h_{\ell}$ are the Yukawa couplings per lepton. Through symmetry breaking $\mathcal{L}_{\text {Yukawa }}$ gives mass terms to the fermions [4]. Extending Equation 2.16 to the whole of the first generation of fermions, the term of the $\mathcal{L}_{\mathrm{SM}}$ responsible for the generation of the masses of the fermions can be written as,

$$
\mathcal{L}_{\text {fermion masses }}=-h_{e} \bar{l}_{L}^{i} \Phi_{i} e_{R}-h_{d}{\overline{q_{L}}}^{i} \Phi_{i} d_{R}-h_{u} \epsilon_{i j} \bar{q}_{L}^{i} \Phi_{i} u_{R}+\text { h.c. }
$$

which will generate mass for the fermions of the from,

$$
m_{\ell}=\frac{h_{\ell} v}{\sqrt{2}}
$$

The final item needed for $\mathcal{L}_{\mathrm{SM}}$, is the SM Higgs doublet and it associated Lagrangian,

$$
\begin{gathered}
\Phi=\frac{1}{\sqrt{2}}\left(\begin{array}{c}
0 \\
v+H
\end{array}\right) \\
\mathcal{L}_{\text {Higgs }}=|\mathbf{D} \Phi|^{2}-\mu^{2} \Phi_{i}^{*} \Phi^{i}+\lambda\left(\Phi_{i}^{*} \Phi_{i}\right)^{2} .
\end{gathered}
$$

where $\mathbf{D}$ is the covariant derivative. The first term in Equation 2.20 will generate interaction terms between the gauge bosons and the Higgs boson. From this term it can be seen that the physical electroweak gauge bosons are superpositions of the $\mathrm{U}(1) \otimes \mathrm{SU}(2)$ bosons. The physical electroweak gauge bosons are defined as, 


$$
\begin{aligned}
& Z_{\mu}=\cos \theta_{W} W_{\mu}^{3}-\sin \theta_{W} B_{\mu} \\
& \gamma=\cos \theta_{W} B_{\mu}+\sin \theta_{W} W_{\mu}^{3} \\
& W^{ \pm}=\frac{\left(W^{1} \mp i W^{2}\right)}{\sqrt{2}}
\end{aligned}
$$

where $\theta_{W}$ is the Weinberg angle or the weak mixing angle, $\tan \theta_{W}=g^{\prime} / g$. Equation 2.20 can now be shown to generate mass terms for the $W$ and $Z$ boson, whereas the photon and gluon remain massless.

$$
M_{W}=\frac{1}{2} g v, \quad M_{Z}=\frac{1}{2} \frac{g v}{\cos \theta_{W}} .
$$

The ratio of the masses of the $W$ boson and the $Z$ boson is therefore predicted in the SM,

$$
\rho=\frac{M_{W}^{2}}{\cos ^{2} \theta_{W}^{2}}
$$

and at tree level this ratio is constrained to be one, with deviations at higher orders [4].

\section{$2.3 \quad$ Asymptotic freedom}

The coupling constants associated with the interactions are not truly constant, their value changes, "runs", with energy. For the electroweak force the coupling increases with increasing energy with the force become strongly coupled at high values. For the strong force the coupling "runs" in the opposite direction, being large at low energies and then decreasing logarithmically. This results in one peculiar property of the strong force, know as asymptotic freedom. This is very relevant for hadronic collisions, as it explains why there are no free quarks and they are only seen confined in bound states. As the coupling between quarks becomes weaker at shorter distances (higher energies), if two quarks are separated 
then the coupling between them becomes stronger, resulting in more and more energy being required try overcome the coupling between them.

This can be viewed as the quarks being connected by a string, made up of gluons and quarks. In trying to separate the quarks this string will stretch, until a certain energy or distance is reached at which point the string will snap, producing more quarks and gluons. These produced quarks will then rebind themselves back into colour neutral objects, into hadrons. This rebinding process is known as hadronization. At small distances when the strong coupling is small, calculations can be performed of the interaction of quarks and gluons using perturbation theory but when the strong coupling becomes large and the strong force strongly interacting then perturbation theory can no longer be used and other methods must be used to calculate the interactions of quarks and gluons. For more details see Chapter 5.

\subsection{Motivation for beyond the Standard Model physics}

The SM has been shown to be a highly successful theory, but there are indications that there are processes not explained by it. For example neutrino masses, though not explicitly included in the theory have been shown by neutrino oscillation experiments to be non-zero [5]. The motivation for extensions arises from both experimental observations, such as explaining dark matter, explaining the observed matter anti-matter asymmetry and explaining the aforementioned neutrino masses and from theoretical motivations, such as solving the Hierarchy problem. The Hierarchy problem arises as the mass of the Higgs boson can acquire very large loop corrections and significant fine tuning of the SM parameters is needed to cancel these corrections. One solution that has been suggested for the 
Hierarchy problem is Supersymmetry, in which a new symmetry between bosons and fermions is hypothesized. The Higgs boson mass loop corrections from the SM particles are then cancelled out by the loop corrections induced by their supersymmetric partners. Such models which introduce new physics beyond the scope of the SM are referred to as beyond the Standard Model, BSM, models.

\subsection{The Supersymmetric Standard Model}

In the Supersymmetric Standard Model, SUSY, for every SM fermion there is introduced a bosonic partner, a sfermion or scalar fermion, and for every SM boson a fermionic partner, a gaugeino. Therefore the particle content is more than doubled as compared to the SM. In SUSY the SM field, $\phi$ and the field of its

supersymmetric partner, $\tilde{\phi}$ can be written together as a superfield, $\hat{\phi}$. With this extension it is also necessary to extend the Higgs sector and in SUSY there are two Higgs doublet superfields, $\hat{H}_{d}$ and $\hat{H}_{u}$, which generate the masses of the downtype and up-type quarks, respectively. This leads to five physical Higgs bosons, two neutral scalars, $h$ and $H$, one neutral pseudoscalar, $A$, and two charged Higgs bosons, $H^{ \pm}$. The minimal version of this theory, the Minimal Supersymmetric Standard Model ,MSSM, contains only these particles but may not be the most attractive form of this set of models. One specific SUSY theory will be discussed, which can be shown to overcome some of the issues that exist with the minimal version.

\subsubsection{NMSSM}

The Next-to-Minimal Supersymmetric Standard Model ,NMSSM, extends the MSSM with the addition of a singlet Higgs superfield, $\hat{S}$. This leads to a extended particle content compared to the MSSM, with three scalar Higgs bosons, $h_{0}, h_{1}$, 
and $h_{2}$, two pseudoscalar Higgs bosons, $a_{1}$ and $a_{2}$ and five neutralinos compared to the four in the MSSM. The NMSSM has been shown to overcome or reduce many problems associated with the MSSM. For example, the NMSSM requires considerably less fine tuning than the MSSM and it provides a solution to the $\mu$ problem of the MSSM. The $\mu$ problem arises in the MSSM since there is nothing to constrain the $\mu$ parameter, the Higgs boson mass parameter, to be of the electroweak breaking scale. In the NMSSM superpotential, the $\mu$ term is replaced by an effective $\mu$ term, $\mu_{e f f}$, which becomes on the order of the SUSY breaking scale when the new singlet field acquires a VEV after the minimization of the scalar potential, therefore constraining this term.

The NMSSM can also be shown to reduce the severity of the Little Hierarchy problem. The Little Hierarchy problem arises as the masses of the SM particles and their supersymmetric partners differ, therefore the radiative corrections to the Higgs boson mass that fix the Hierarchy problem do not completely cancel. If the supersymmetric partners are much heavier than their SM partners the Little Hierarchy problem can introduce the need for considerable fine tuning. In the NMSSM the Higgs boson to Higgs boson couplings can dominate, for example, the decay of the SM-like Higgs to a pair of $a_{1}$ bosons, which can dominate over its decay into a $b \bar{b}$ pair. Therefore the limits set by LEP on the mass of the SM-like Higgs boson can be as low as $87 \mathrm{GeV}$ [6], allowing for a light SM Higgs boson of approximately $100 \mathrm{GeV}$ as preferred by the electroweak precision data. With this lower allowed mass of the SM-like Higgs boson the need for fine tuning is reduced.

The superpotential of the NMSSM, expressed in terms of superfields is [7],

$$
W_{\mathrm{NMSSM}}=W_{\mathrm{MSSM}}-\lambda \hat{S} \hat{H}_{u} \hat{H}_{d}+\frac{1}{3} \kappa \hat{S}^{3}
$$

where $\hat{S}$ is the singlet Higgs superfield, $\lambda$ and $\kappa$ are dimensionless couplings 
governing the interactions of the singlet Higgs superfield. In writing the superpotential of the NMSSM, the fermion generation, $\mathrm{SU}(2)$ and colour indices have been suppressed. The superpotential of the MSSM is

$$
W_{\mathrm{MSSM}}=\left[h_{e} \hat{H}_{d} \hat{L} \hat{E}+h_{d} \hat{H}_{d} \hat{Q} \hat{D}+h_{u} \hat{H}_{u} \hat{Q} \hat{U}\right]
$$

where $\hat{L}$ and $\hat{Q}$ are the SU(2) doublets containing, respectively, left handed leptons and their superpartners, and left handed quarks and squarks. $\hat{E}, \hat{U}$, and $\hat{D}$ are singlets containing the right handed leptons, up-type quarks, and downtype quarks, respectively. The lepton, down-type, and up-type quark Yukawa couplings are $h_{e}, h_{d}$, and $h_{u}$, respectively.

\subsection{Doubly charged Higgs bosons}

Higgs bosons with a double charge appear in numerous extensions to the SM, such as Little Higgs models [8] and Left-Right symmetric models [9, 10]. They can be generated either in models which have both a Higgs doublet with $Y=3$ and a neutral Higgs doublet [11], or in models which have Higgs triplets. It has been shown that doubly charged Higgs, $H^{ \pm \pm}$, bosons could have masses in the range accessible to current colliders, for example through the See-Saw mechanism [12]. For doubly charged Higgs bosons that appear in models with a triplet or higher representations with an additional neutral member of the Higgs sector, there are strong constraints that arise from ensuring that $\rho=1$ at tree level (Equation 2.23). This constraint can be overcome by requiring that the models do not have a neutral member or by requiring that the VEV of this member is zero or vanishingly small [11]. This requirement results in such models not having a $W^{+} W^{-} \rightarrow H^{ \pm \pm}$coupling. Comparatively the decay $Z / \gamma^{*} \rightarrow H^{++} H^{--}$ is allowed in all models [11]. 


\subsubsection{Left-Right symmetric models}

Left-Right symmetric models are an extension to the SM that predict a new symmetry between left and right handed particles $[9,10]$ and provide a way of introducing neutrino masses. The model extends the electroweak sector of the $\mathrm{SM}$ to $\mathrm{SU}(2)_{L} \otimes \mathrm{SU}(2)_{R} \otimes \mathrm{U}(1)$, introducing a second $\mathrm{SU}(2)$ sector for the right handed electroweak sector. This model adds both an additional right handed neutrino and right handed partners for the SM bosons. The Higgs sector is extended to generate masses for these new particles, containing at least one bidoublet and both a left and a right handed triplet. These triplets are chosen so they can generate masses for the right handed neutrinos through the See-Saw mechanism [12]. The Higgs bi-doublet $\phi$ and the two triplets $\Delta_{L, R}$ can be written as

$$
\phi=\left(\begin{array}{cc}
\phi_{1}^{0} & \phi_{1}^{+} \\
\phi_{2}^{-} & \phi_{2}^{0}
\end{array}\right), \Delta_{L, R}=\left(\begin{array}{cc}
\frac{\Delta_{L, R}^{+}}{\sqrt{2}} & \Delta_{L, R}^{++} \\
\Delta_{L, R}^{0} & \frac{\Delta_{L, R}^{+}}{\sqrt{2}}
\end{array}\right),
$$

and their corresponding VEVs,

$$
\langle\phi\rangle=\left(\begin{array}{cc}
\frac{k 1}{\sqrt{2}} & 0 \\
0 & \frac{k 2}{\sqrt{2}}
\end{array}\right),\left\langle\Delta_{L, R}\right\rangle=\left(\begin{array}{cc}
0 & 0 \\
\frac{v_{L, R}}{\sqrt{2}} & 0
\end{array}\right),
$$

$k_{1,2}$ are the VEV's of bi-doublet and break the SM symmetry, providing masses to $W_{L}$ and $Z$ bosons and the left handed fermions, and are of the order of the electroweak scale [12]. For the VEVs of the triplet, $v_{R}$ provides masses for new

right handed gauge bosons, $W_{R}$ and $Z^{\prime}$ and a right handed neutrino $\nu_{R}$, these new particles could be very heavy, implying that $v_{R}$ may also be large. Whereas $v_{L}$ contributes to the value of the $\rho$ parameter (Equation 2.23) and therefore must be small. The value of $v_{L}$ has been shown to be experimentally constrained to values $\leq 5 \mathrm{GeV}[13]$

This model predicts neutral Higgs bosons, charged Higgs bosons and doubly 
charged Higgs bosons. As with all particles in this theory there is both a left and a right handed state, and hence a left and a right handed doubly charged Higgs boson, $H_{\mathrm{L}}^{ \pm \pm}$and $H_{\mathrm{R}}^{ \pm \pm}$. As the Higgs boson is a spin zero particle the handedness referes to the particles it couples to, not to the alignment of its own spin. For the $H_{\mathrm{L}}^{ \pm \pm}$boson the pair production cross section is predicted to be approximately a factor of two higher than for the $H_{\mathrm{R}}^{ \pm \pm}$boson, due to their different coupling to the $Z$ boson [9].

\subsubsection{Little Higgs models}

Little Higgs models $[14,15,16]$ are a class of models which use the introduction of new strongly interacting dynamics at a high energy scale to overcome the Little Hierarchy problem. In Little Higgs models the SM Higgs boson is a light pseudoGoldstone boson of a broken global symmetry. The concept of non-zero neutrino masses within these models has been extensively studied $[17,18,19,20,21]$ and naturally occurs through the See-Saw mechanism, when a Higgs triplet is introduced

These models predict new heavy gauge bosons, $W_{H}$ and $Z_{H}$, a heavy quark pair and new Higgs bosons, including doubly charged Higgs bosons. The small measured value for the neutrino masses implies that the VEV of the doubly charged Higgs boson in Little Higgs models, will be small, resulting in the decays $H^{ \pm \pm} \rightarrow W^{ \pm} W^{ \pm}$being negligible [22]. The coupling of the $H^{ \pm \pm}$boson to leptons in Little Higgs models is dependent not on the size of the Yukawa couplings to $H^{ \pm \pm}$bosons, which are not known, but on their ratios. These ratios have been measured in neutrino oscillation measurements, and are known to take one of two possible forms, the normal hierarchy of neutrino masses or the inverted hierarchy of neutrino masses. Assuming the normal hierarchy and a very small mass for the lightest neutrino, as implied by neutrino experiments, then the decay 
of $H^{ \pm \pm}$bosons to electrons can be neglected and Little Higgs models predict that the branching ratio, $\mathcal{B}$, of $H^{ \pm \pm}$bosons to $\mu \mu, \tau \tau$, and $\mu \tau$ lepton pairs are approximately equal, $\mathcal{B}\left(H^{ \pm \pm} \rightarrow \mu \mu\right)=\mathcal{B}\left(H^{ \pm \pm} \rightarrow \mu \tau\right)=\mathcal{B}\left(H^{ \pm \pm} \rightarrow \tau \tau\right) \approx$ $1 / 3[22]$.

\subsection{Searches for Higgs bosons}

\subsubsection{Standard Model Higgs bosons}

The Higgs mechanism provides a way of giving masses to the $W$ and $Z$ bosons and to the fermions through Yukawa interactions. In the SM its mass is given by $M_{H}=\sqrt{2 \lambda} v$ where $\lambda$ is the Higgs boson self-coupling the value of which is unknown and $v$ is the VEV which is fixed at $246 \mathrm{GeV}$ by the Fermi coupling, $G_{F}$. Theoretical constraints based on the scale of new BSM physics restrict $M_{H}$ to be around $130 \mathrm{GeV}$ to $180 \mathrm{GeV}[23]$. Though $M_{H}$ is not known, the couplings to the SM particles are, with the coupling of the Higgs bosons to fermions being proportional to their masses and the coupling of the Higgs bosons to gauge bosons being proportional to their squared mass. The predicted decays of the SM Higgs boson to fermions and gauge bosons are shown in Figure 2.3. It can be seen at low $M_{H}$ that the main decays are to fermions, $b$ quarks and $\tau$ leptons, and at high $M_{H}$ to bosons.

The dominant production mechanism for the SM Higgs boson at both the Tevatron and the LHC is gluon-gluon fusion, $g g \rightarrow H$. With the other dominant channels being, the associated production of a Higgs boson with a $W$ or $Z$ boson or with top quark pairs, and vector boson fusion, $q q \rightarrow q q H+X$. The Higgs boson production modes for both the LHC and the Tevatron as a function of the Higgs mass are shown in Figure 2.4.

Searches have been performed for the SM Higgs boson at LEP, at the Tevatron, 


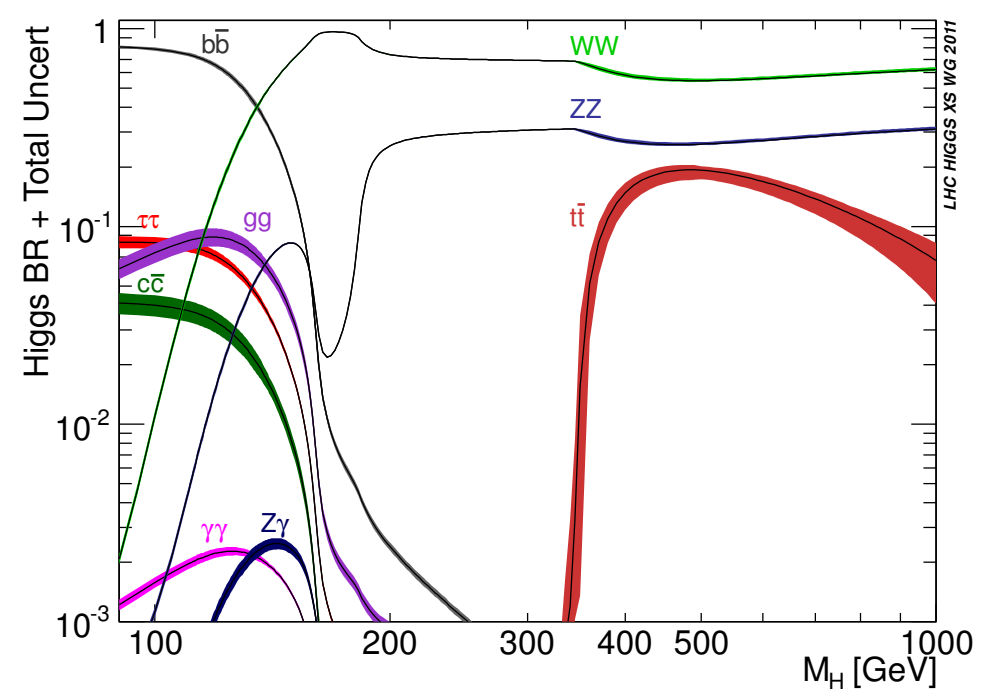

Figure 2.3: The predicted decays of the SM Higgs boson as a function of its mass [23].

and at the LHC. The combined search sensitivity of the four experiments at the LEP collider was up to approximately $115 \mathrm{GeV}$ and limits were set with a lower bound of $M_{H}>114.4 \mathrm{GeV}$ at $95 \%$ C.L. [24]. At the Tevatron collider $M_{H}$ between 90 to $200 \mathrm{GeV}$ can be probed [23] and searches are most sensitive, for $M_{H}=125 \mathrm{GeV}$, to a Higgs boson produced through the associated production of a Higgs boson with a $W$ or $Z$ boson, where the Higgs decays into bottom quarks. At masses greater than $135 \mathrm{GeV}$ the $H \rightarrow W W$ decay channel dominates, with the Higgs boson being predicted to be produced mainly through the associated production and gluon-gluon fusion production channels. The results from both the DØ [1] and the CDF [25] experiments are combined to produce Tevatron exclusion limits on the SM Higgs boson cross section, as can be seen in Figure 2.5, produced with total integrated luminosity of $10 \mathrm{fb}^{-1}$. The Tevatron has excluded mass regions between 100 to $106 \mathrm{GeV}$ and 147 to $179 \mathrm{GeV}$ [2]. A SM Higgs boson-like excess is also seen in the range $115<M_{H}<135 \mathrm{GeV}$. This excess has a significance of 3.0 standard deviations, $\sigma$. This significance is reduced if the 
LHC

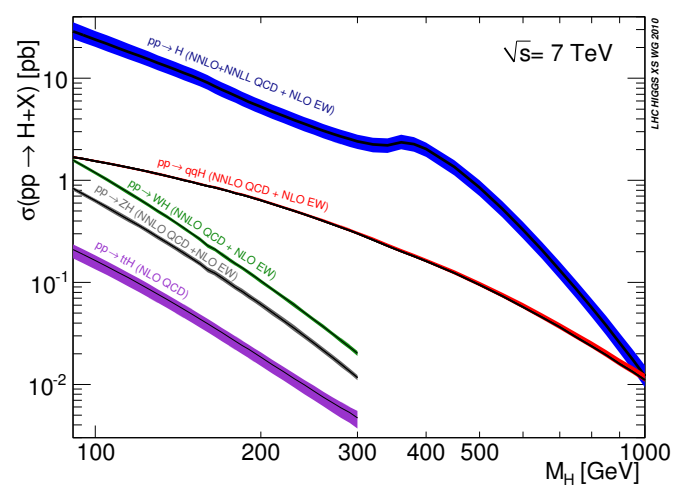

Tevatron

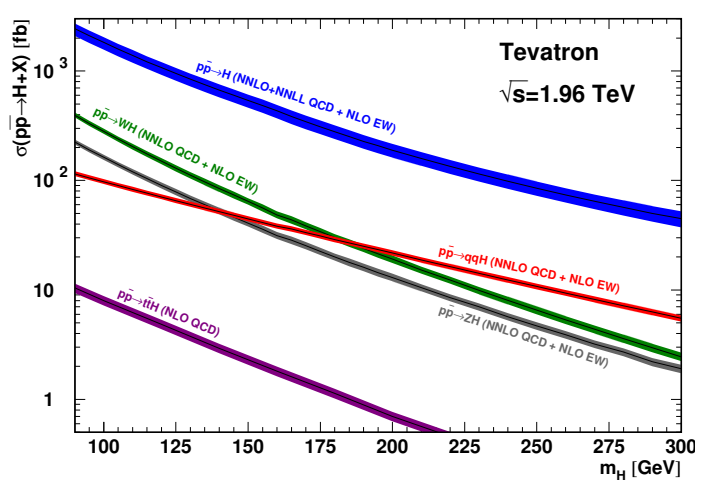

Figure 2.4: The SM Higgs boson production mechanism at the LHC (left) and Tevatron (right) as a function of the Higgs mass. The contribution from gluongluon fusion is shown in blue, $W H$ associated production in green, $Z H$ associated production in grey, vector boson fusion in red, and $t \bar{t} H$ associated production in purple [23].

"look-else-where effect" is included, which takes into account that there is a range of parameter space being studied where a statistically significant excess could be seen by chance [26]. The significance without this effect is referred to as the local significance and with it the global significance. Once this effect is taken into account the significance as seen by the Tevatron is reduced to $2.5 \sigma$. This excess has been seen to be dominated by the searches in the decay channel, $H \rightarrow b b[27]$. When considering only this channel a local significance of $3.2 \sigma$ is observed and a global significance of $2.9 \sigma$. The $H \rightarrow W W$ channel is not observed to have such an excess but is not inconsistent with it [28]. The comparison of the results seen in these decay channels with the predicted SM couplings for $M_{H}=125 \mathrm{GeV}$ are shown in Figure 2.6.

At the LHC, the higher collision energies and the use of a proton-proton beam compared to the proton-antiproton used at the Tevatron, means the dominant production mechanism differ, as shown in Figure 2.4. For the decay mechanisms, the most sensitive channels at the LHC are, $H \rightarrow \gamma \gamma$ for $M_{H}<120 \mathrm{GeV}$ and 


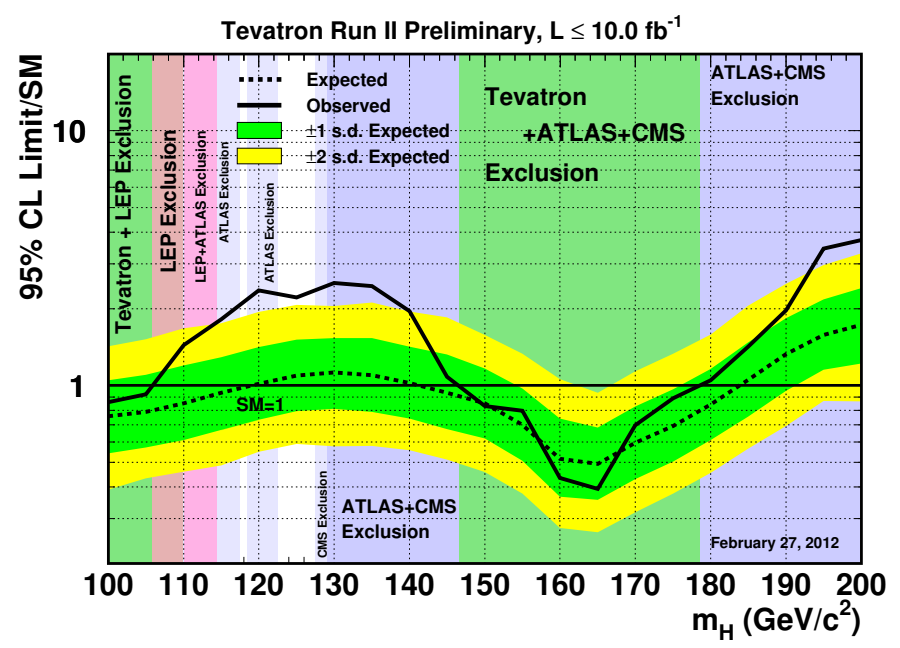

Figure 2.5: The Tevatron Higgs boson cross section limits as a function of the SM Higgs boson mass. The cross section limits are given as the ratio to the predicted SM Higgs cross section. The observed cross section limit is shown by the solid black line and the expected cross section limit by the dotted back line. The one and two standard deviation bands on the expected cross section, are shown in green and yellow, respectively. The exclusion regions determined by the different experiments are also shown [2].

for $M_{H}>120 \mathrm{GeV} H \rightarrow W W$ and $H \rightarrow Z Z$. In July 2012, new results were released from both the ATLAS and CMS experiments, showing evidence for a new boson which is consistent with the SM Higgs boson. These results were based both on the 2011 data with $\sqrt{s}=7 \mathrm{TeV}$ and the 2012 data with $\sqrt{s}=8$ $\mathrm{TeV}$, and amounted to approximately $10 \mathrm{fb}^{-1}$ of integrated luminosity. Both experiments published the results independently showing consistent results. These results where dominated by two channels, the $H \rightarrow Z Z[29]$ and $H \rightarrow \gamma \gamma[30]$ decay modes. The ATLAS experiment results observed an SM Higgs boson-like excess with an local significance of $5.0 \sigma$, centred at $126.5 \mathrm{GeV}$, with an expected significance of $4.6 \sigma$ [31]. The CMS experiment observed an SM Higgs boson-like excess with an local significance of $4.9 \sigma$, with an expected significance of $5.9 \sigma$. CMS has measured the mass of the boson corresponding to this excess to be 


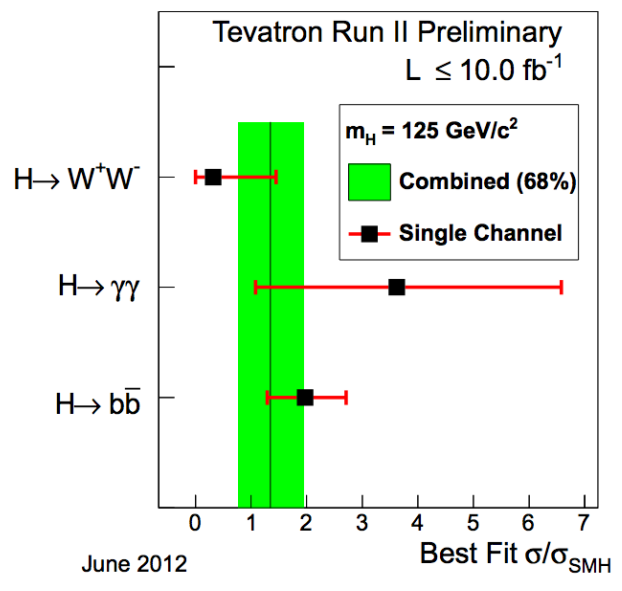

Figure 2.6: The best fit signal strength for the Tevatron Higgs boson searches compared to the SM predictions, where the black line shows the combined fit, the red lines the fits for $H \rightarrow b b, H \rightarrow W W$, and $H \rightarrow \gamma \gamma$ decay channels. The green band show the $68 \%$ uncertainty on the combined fit [2].

$125 \pm 0.6 \mathrm{GeV}[32]$. The comparison of the ATLAS and CMS SM Higgs boson search results compared to the predicted couplings from the SM are shown in Figure 2.7 and the results in all channels can be seen to be consistent with a SM Higgs boson.

\subsubsection{Doubly charged Higgs bosons}

Both direct and indirect searches have been performed for doubly charged Higgs bosons.

\section{Direct searches}

Searches have been performed by the LEP collaborations in the $e^{+} e^{-} \rightarrow H^{++} H^{--}$ channel $[33,34,35]$, looking for four prompt leptons in the final state. Limits on the $H^{ \pm \pm}$boson mass within the range of 95 to $100 \mathrm{GeV}$ were determined for Left-Right symmetric models, with the exact values depending on the flavour 
CMS

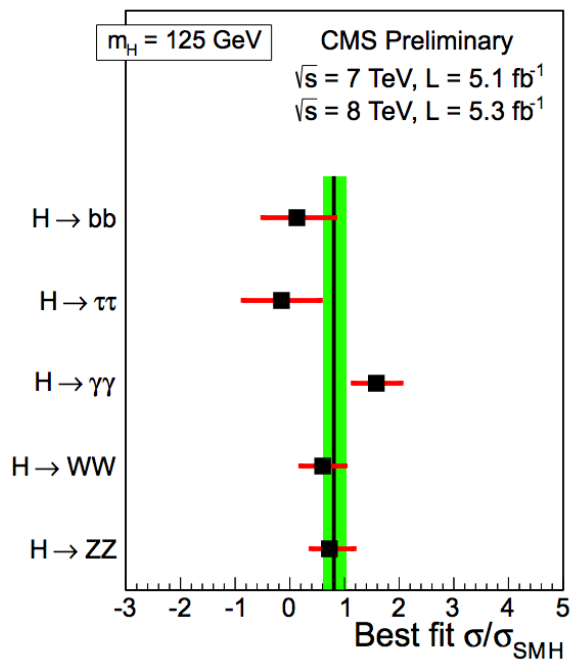

ATLAS

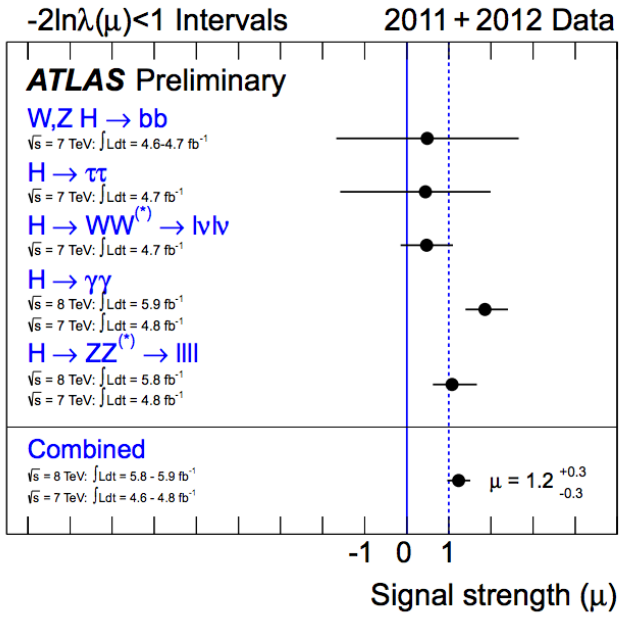

Figure 2.7: The best fit signal strength for both the ATLAS (right) and CMS (left) Higgs boson searches compared to the SM predictions. For CMS the black line shows the combined fit, the red lines the fits for $H \rightarrow b b, H \rightarrow \tau \tau, H \rightarrow W W$, $H \rightarrow \gamma \gamma$, and $H \rightarrow Z Z$ channels. The green band show the $68 \%$ uncertainty on the combined fit [32]. For ATLAS the blue line shows the SM prediction and the dotted line the best fit. $\mu$ is defined as, $\mu=\sigma / \sigma_{\text {SM Higgs }}[31]$.

of the final state leptons. The OPAL collaboration also performed a search for single $H^{ \pm \pm}$boson production, $e^{+} e^{-} \rightarrow e^{\mp} e^{\mp} H^{ \pm \pm}[36]$.

At the Tevatron, both $\mathrm{D} \varnothing$ and CDF have performed searches for pair produced $H^{ \pm \pm}$bosons, $p \bar{p} \rightarrow H^{++} H^{--}$, decaying leptonically. D $\varnothing$ has searched for the four muon final state and set mass limits of $M\left(H_{\mathrm{L}}^{ \pm \pm}\right)>150 \mathrm{GeV}$ and $M\left(H_{\mathrm{R}}^{ \pm \pm}\right)>127 \mathrm{GeV}$ for the right and left handed states, respectively [37]. The CDF experiment has searched for $H^{++} H^{--}$decays with a $100 \%$ branching ratio into pairs of $e e, \mu \mu, e \mu, e \tau$ and $\mu \tau$ leptons and set limits of between $M\left(H_{\mathrm{L}}^{ \pm \pm}\right)>112$ to $M\left(H_{\mathrm{L}}^{ \pm \pm}\right)>136 \mathrm{GeV}$ for a left handed $H^{ \pm \pm}$boson dependent on the state studied. Right handed $H^{ \pm \pm}$mass limits were only set for the decay into $\mu \mu$ lepton pairs, at $M\left(H_{\mathrm{R}}^{ \pm \pm}\right)>113 \mathrm{GeV}$. In addition CDF performed a search for long lived $H^{ \pm \pm}$bosons that would decay outside the detector, setting limits for 
left and right handed states of $M\left(H_{\mathrm{L}}^{ \pm \pm}\right)>133 \mathrm{GeV}$ and $M\left(H_{\mathrm{R}}^{ \pm \pm}\right)>109 \mathrm{GeV}$, respectively [38]. A summary of the LEP and Tevatron lower mass limits on the $H^{ \pm \pm}$boson is shown in Figure 2.8.

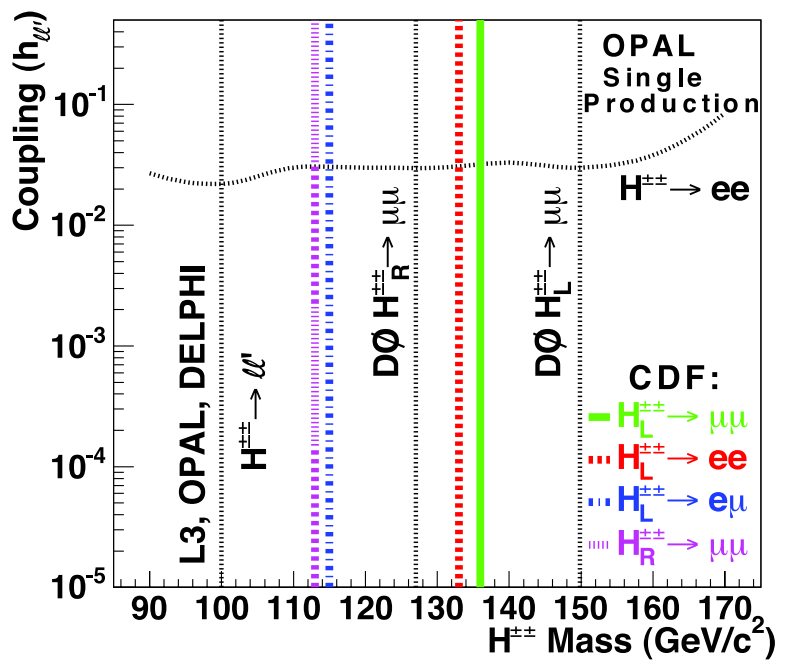

Figure 2.8: The LEP and Tevatron lower mass limits on the coupling of the doubly charged Higgs boson to leptons as a function of mass [39].

More recently, there have been results from the LHC. CMS searched for pair or signally produced doubly charged Higgs bosons, $p p \rightarrow H^{++} H^{--}$or $p p \rightarrow H^{++} H^{-}$, decaying to $\ell \ell^{\prime}$ pairs where $\ell$ and $\ell^{\prime}$ can be $e, \mu$, or $\tau$. No significant excess was seen in the data over the predicted SM background events and upper mass limits were set in the range $M\left(H_{\mathrm{L}}^{ \pm \pm}\right)>165$ to $457 \mathrm{GeV}$, for the different production and decay modes of the $H^{ \pm \pm}$boson studied [40]. The ATLAS experiment searched for both left and right handed $H^{ \pm \pm}$bosons assuming a $100 \%$ decay to muons. Limits on the mass at $M\left(H_{\mathrm{L}}^{ \pm \pm}\right)>355 \mathrm{GeV}$ for left handed states and $M\left(H_{\mathrm{R}}^{ \pm \pm}\right)>251 \mathrm{GeV}$ for the right handed [41] are obtained. 


\section{Indirect searches}

Indirect searches can constrain the Yukawa coupling of the $H^{ \pm \pm}$boson. Limits can be set from the non-observation of flavour violating leptonic decays, these include searches for muonium $\left(\mu^{+} e^{-}\right)$to anti-muonium $\left(\mu^{-} e^{+}\right)$transitions, $\left(e^{+} e^{-} \rightarrow \mu \mu\right)$ [42] and from searches for rare muon decays, $\mu \rightarrow e e e$ and $\mu \rightarrow e \gamma$.

Doubly charged Higgs bosons would also contribute to the amplitude of various SM processes, where the deviation from the predicted SM value can be used to set limits on the coupling of the $H^{ \pm \pm}$boson. These include Bhabha scattering $e^{+} e^{-} \rightarrow e^{+} e^{-}$, which was searched for at LEP [36] and effects on the anomalous magnetic moment of the muon. The value of g-2 measured by Brookhaven [43] was seen to deviate from the predicted value and this deviation can be interpreted as being due to a $H^{ \pm \pm}$boson, allowing one to set limits on its coupling [44].

\section{Searching for doubly charged Higgs bosons at the Tevatron}

Doubly charged Higgs bosons can be produced through three production mechanisms at the Tevatron, $p \bar{p} \rightarrow Z / \gamma^{*} \rightarrow H^{++} H^{--}, p \bar{p} \rightarrow W^{ \pm} \rightarrow H^{ \pm \pm} H^{\mp}$, or $p \bar{p} \rightarrow W^{ \pm} W^{ \pm} \rightarrow H^{ \pm \pm}$. The existing phenomenological and theoretical constraints favour the $W^{ \pm} W^{ \pm} \rightarrow H^{ \pm \pm}$coupling to be zero or vanishing [11]. The pair production $H^{ \pm \pm} H^{\mp}$ channel is model dependent, whereas the decay to a $H^{ \pm \pm} H^{\mp}$ boson pair is not. The possible decays of the doubly charged Higgs boson are either $H^{ \pm \pm} \rightarrow \ell^{ \pm} \ell^{ \pm}$(same electric charge lepton pairs), $H^{ \pm \pm} \rightarrow H^{ \pm} W^{ \pm}$ or $H^{ \pm \pm} \rightarrow H^{ \pm} H^{ \pm}$. The $H^{ \pm}$and $H^{ \pm \pm}$are typically expected to have similar masses so the decay $H^{ \pm \pm} \rightarrow H^{ \pm} H^{ \pm}$is not favored [11].

Which of the first two decay modes is dominant depends on the coupling of the leptons to the $H^{ \pm \pm}$boson. Limits have been set from the indirect searches as described in the previous section. These suggest that off diagonal couplings are small and set limits on the $e e$ and $\mu \mu$ lepton couplings, of order $\leq 10^{-5}$ [11]. If the 
coupling of the doubly charged Higgs to leptons is of this order, then the dominant doubly charged Higgs boson decay will be to same sign lepton pairs [11]. There are no limits on the tau lepton coupling, so this could easily be the dominant decay. The next weakest limit is set on the coupling of the $H^{ \pm \pm}$boson into muons. Since the Higgs boson might prefer to decay to heavier particles, it is reasonable to assume that the $\mu$ and $\tau$ lepton decay modes will be the dominant decay modes, with the coupling of the $H^{ \pm \pm}$bosons to electrons being small. 


\section{Chapter 3}

\section{Experimental apparatus}

The Tevatron is a proton anti-proton collider at the Fermi National Accelerator Laboratory in Illinois, USA. The collider has been in operation since 1986 and ran until 2011 when it was shut down permanently. The running of the Tevatron is split into two periods, Run I from 1986 to 1996 and Run II from 2001 to 2011. At the end of Run I the Tevatron was shut down for major upgrades, including the centre of mass energy being increased from $\sqrt{s}=1.8 \mathrm{TeV}$ to $\sqrt{s}=1.96 \mathrm{TeV}$ and the instantaneous luminosity being increased by an order of magnitude to $10^{32} \mathrm{~cm}^{-2} \mathrm{~s}^{-1}$. Details about the collider and its subsystems are given in Section 3.1 and the layout of the different subsystems are shown in Figure 3.1. The Tevatron has two general purpose detectors which recorded the particles created in the proton anti-proton collisions, the Central Detector at Fermilab, CDF, and the $\mathrm{D} \varnothing$ detector. Details of the $\mathrm{D} \varnothing$ detector are given in Section 3.2.

\subsection{The Tevatron}

The Tevatron Collider and its associated subsystems will be described here. 


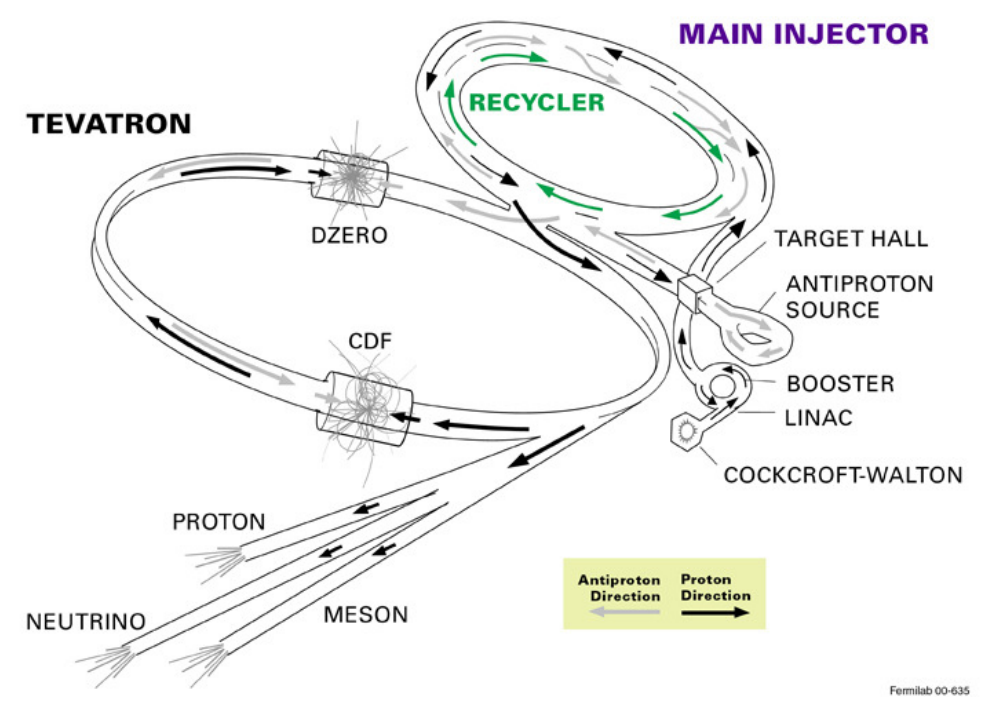

Figure 3.1: Diagrammatic representation of the Tevatron ring and subsystems used for the different stages of beam production for the Tevatron Collider. Also shown are the proton, meson, and neutrino beams that are sent to fixed target experiments [45].

\subsubsection{Proton Source}

The first step in colliding the proton and anti-proton beams is to produce a pure proton beam that can be accelerated. To achieve this the Cockcroft-Walton pre-accelerator, a hydrogen source inside a charged dome, is used. The source ionizes the hydrogen gas to produce charged hydrogen ions. The dome is held at a potential difference of $-750 \mathrm{kV}$, allowing the ions to accelerate from the charged dome to a grounded wall resulting in a $750 \mathrm{keV}$ beam [46]. The ions are then passed to a linear accelerator or Linac.

\subsubsection{Linac}

Within the Linac, bunches of these hydrogen ions are accelerated further to $400 \mathrm{GeV}$, using a series of radio frequency, RF, cavities [46]. Within the Linac, 
the bunches of protons are passed through periodic gaps or cavities in the beam pipe, which contain an electric field at a frequency such that the particles experience an acceleration as they pass through. The Linac consists of two sections, a low energy drift tube Linac and a high energy side coupled cavity Linac [46] and is approximately 150 metres in length.

\subsubsection{Booster}

Inside the Booster, the hydrogen ions pass through carbon foil which strips them of their electrons creating a pure proton beam. The Booster is a small synchrotron, or circular accelerator, with a $75 \mathrm{~m}$ radius, which increases the energy of the proton beam to $8 \mathrm{GeV}$. The Booster uses a magnetic field to keep the particles on their course and has $19 \mathrm{RF}$ cavities to accelerate the particles. Unlike in the Linac, where particles only travel through each RF cavity once, particles are repeatedly passed through these cavities in a synchrotron, each time getting additional acceleration, resulting in large accelerations being achieved. RF cavities also serve an additional purpose in a synchrotron as particles arriving in the cavity slightly before, or after, those in phase will receive slightly larger, or smaller, accelerations and hence a slightly larger, or smaller, increase in their rotational frequency. This helps to ensure that all particles remain in a stable orbit over many cycles [47].

\subsubsection{Main Injector}

From the Booster the protons are passed to the Main Injector. The Main Injector is a synchrotron containing $18 \mathrm{RF}$ cavities and has a circumference of $3.3 \mathrm{~km}$. It takes the $8 \mathrm{GeV}$ beams from the Booster and accelerates them to either $150 \mathrm{GeV}$ or $120 \mathrm{GeV}$. The $150 \mathrm{GeV}$ beam will be passed to the Tevatron for further acceleration and the $120 \mathrm{GeV}$ beam is used to create the anti-proton beam. It 
also accepts an $8 \mathrm{GeV}$ anti-proton beam from the Recycler that it accelerates to $150 \mathrm{GeV}$, ready to be injected in the Tevatron.

\subsubsection{Anti-proton Source}

The $120 \mathrm{GeV}$ proton beam from the Main Injector is focused on a nickel alloy target causing interactions that produce anti-protons. The anti-protons created in these collisions, with an energy of approximately $8 \mathrm{GeV}$, are passed to the Debuncher ring. Only a miniscule number of anti-protons are produced for the number of incident protons, about 20 anti-protons for $10^{6}$ protons incident on the target. The numerous unwanted particles that are produced in this process, which are either not anti-protons or are anti-protons not in the correct energy range, are sent to a beam dump. Magnets are used to select particles whose momentum and charge correspond to an $8 \mathrm{GeV}$ anti-proton beam.

\section{Debuncher and Accumulator}

The Debuncher ring is designed to create an anti-proton beam ready to be accelerated. The Debuncher is a triangular synchrotron, which uses RF manipulation to control the momentum spread of the anti-protons from the target. From the Debuncher ring the anti-proton beam is passed to the Accumulator where it is stored and cooled. The Accumulator is also a triangular synchrotron located in the same ring as the Debuncher and from the Accumulator the anti-protons are passed to the Recycler.

\subsubsection{Recycler}

The Recycler is also a storage ring for the anti-protons located in the same ring as the Main Injector. It does not accelerate the anti-protons but stores and cools them further than the Accumulator is capable of doing, keeping them at $8 \mathrm{GeV}$. It 
has two cooling systems, a stochastic cooling system used at low intensities, and an electron cooling system used when there are more than $200 \times 10^{10}$ anti-protons in the Recycler. Electron cooling works by having an additional beam of "cool" electrons circulating with the anti-protons. Collisions between the two transfers momentum from the "hot" protons to the electrons [46]. From the Recycler the anti-proton beam is passed back to the Main Injector where it is accelerated to $150 \mathrm{GeV}$.

\subsubsection{Tevatron Collider}

The $150 \mathrm{GeV}$ proton and anti-proton beams from the Main Injector are passed to the Tevatron Collider. Here they are accelerated to their final energy of $980 \mathrm{GeV}$ each, therefore producing collisions at a centre-of-mass energy of $\sqrt{s}=1.96 \mathrm{TeV}$. The Tevatron Collider is a circular synchrotron, $6.3 \mathrm{~km}$ in circumference, with 8 RF cavities. Each round of proton anti-proton injections is known as a "store". For each store groups, or bunches, of protons and anti-protons are injected into the Tevatron. In total 36 bunches are injected, with an equal number of proton and anti-proton bunches with a spacing of 396 ns between bunches. Due to the high availability of protons, the proton bunches are three times larger at $150 \times 10^{9}$ protons per bunch, compared to $50 \times 10^{9}$ anti-protons per bunch.

The bunches are guided around the Tevatron beam pipe in opposite directions, kept on path and focused by over 1000 superconducting niobium/titanium magnets. The superconductive magnets are cooled to $4 \mathrm{~K}$, so they remain superconducting, allowing for high currents to be used [46]. The Tevatron uses several types of magnets for different purposes. Dipole magnets keep the particles on their course within the beam pipe and quadrupole magnets focus the particles. Additional correctional magnets allow for very small corrections or focusing.

The beams of protons and anti-protons are designed to collide at the location 
of the CDF and $\mathrm{D} \varnothing$ detectors (see Figure 3.1). The name $\mathrm{D} \varnothing$ comes from the detectors position around the ring. The Tevatron ring is divided into 6 sections, A through F, which are further divided into buildings 0 to 4 . CDF is located at $\mathrm{B} 0$ and $\mathrm{D} \varnothing$ at D0.

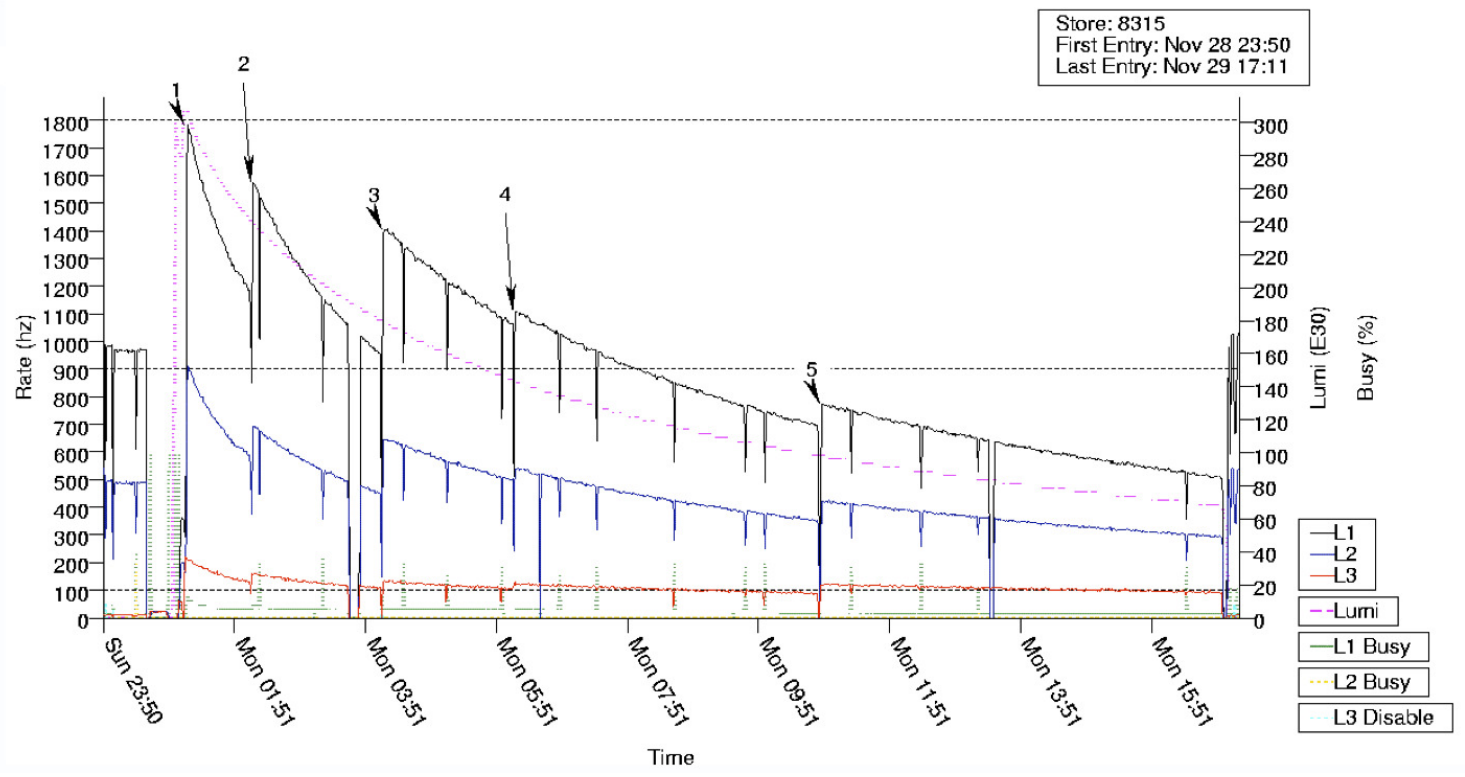

Figure 3.2: A typical store at D $\varnothing$, showing the instantaneous luminosity (right) and trigger rate (left), as a function of time. The total instantaneous luminosity is shown in purple and the three levels of the trigger system in black, blue and red.

At a start of a store there is a high instantaneous luminosity, at around $4 \times 10^{32} \mathrm{~cm}^{-2} \mathrm{~s}^{-1}$, this decreases over the period of the store as particles are lost in collisions and other interactions. A store was generally on the order 12 hours, as can be seen in Figure 3.2. The structure of this plot is described in Section 4.1 which describes the triggering system at $D \varnothing$. 


\subsection{The $\mathrm{D} \emptyset$ detector}

The DØ detector was proposed in 1983 and operated from April 1992 to February 1996 at which point it was shutdown for significant improvements, both to improve on its ability to detect and measure the properties of particles, and in order to deal both with the increased energy and luminosities of Run II (June 2006 to September 2011). Unless otherwise stated the description given below will described the detector after this upgrade. Over its period of operations it recored $10.7 \mathrm{fb}^{-1}$ of integrated luminosity, out of the $11.9 \mathrm{fb}^{-1}$ delivered by the Tevatron.

The $\mathrm{D} \varnothing$ detector is a general purpose detector with, working out from the beam pipe; a central tracking system, consisting of both a silicon strip and a scintillating fiber tracker; an electromagnetic and hadronic calorimeter; a muon spectrometer; and two magnets, a solenoid between the inner tracker and the calorimeters and a toroid magnet located between the calorimeters and muon system. The layout of the components can be seen in Figure 3.3 and are described in detail in the following sections. Collisions within the detector will be, approximately symmetric around the collision point, therefore the detector and its subsystems are designed to reflect and show this symmetry. The triggering system, electronics, and computers that process the information from these systems and determine which events should be recorded are described in Section 4.1.

Figure 3.4 shows a schematic of how different types of particles will react in the detector. Charged particles will be observed in the central tracking systems, particles that undergo electromagnetic interactions, will deposit the majority of their energy in the EM calorimeters and those that undergo hadronic interaction, will deposit the majority of their energy in the hadronic calorimeter. Muons are minimally ionizing particles hence leave little evidence of themselves in the calorimeters. They are observed in the specialized muon systems. Taking the 


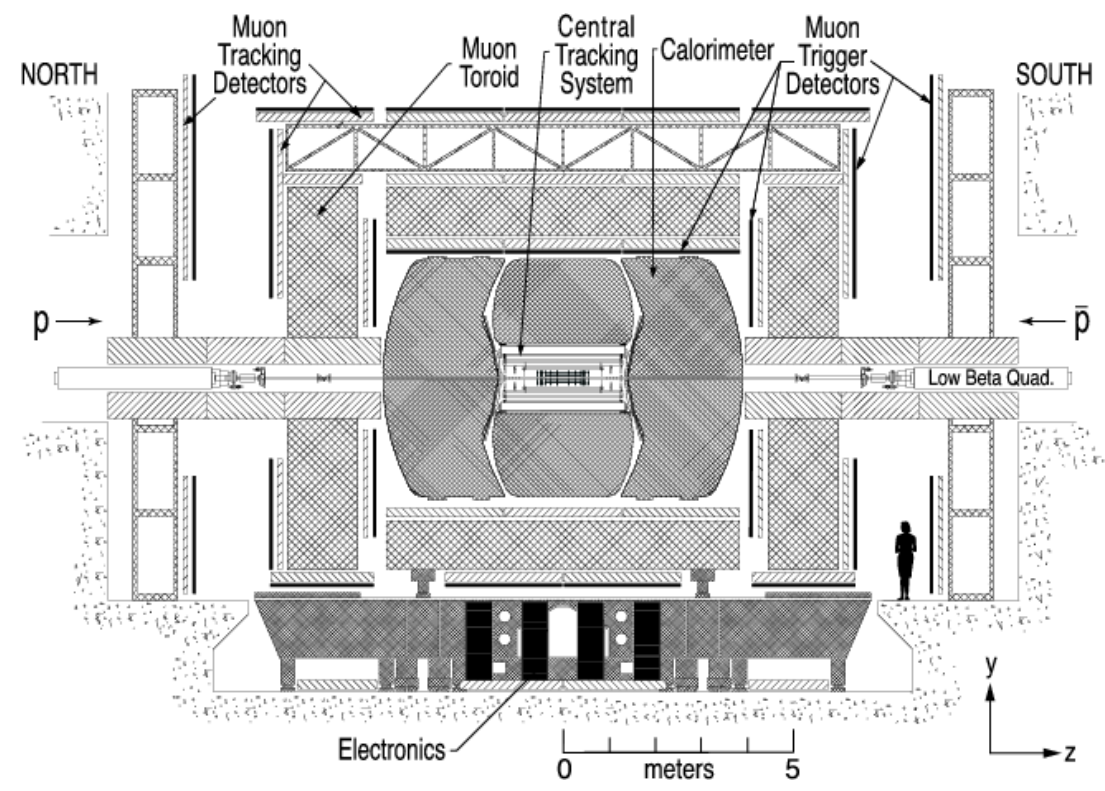

Figure 3.3: Diagrammatic representation of the $\mathrm{D} \varnothing$ detector. The paths of the protons and anti-protons are labeled as $p$ and $\bar{p}$, respectively, with an arrow showing the direction of the particle beams [48].

information from all these components of the detector allows one to reconstruct what types of particles have been created in a collision and the properties of those particles; i.e. energy, momentum, and position in the detector. This reconstruction process is described in Section 4.2.

The following description uses a right handed coordinate system, where the $z$-axis is taken to be along the proton direction and the $y$-axis is taken to be upwards, see Figure 3.3. The azimuthal and polar angles are denoted as $\phi$ and $\theta$, respectively. The perpendicular distance, $r$, from the $z$-axis is given by $r=$ $\sqrt{x^{2}+y^{2}}$. The term "forward" is used to denote regions of the detector at large detector $\eta$, where $\eta$ is the pseudorapidity, defined as

$$
\eta=-\ln [\tan (\theta / 2)]
$$

this pseudorapidity is an approximation of the true rapidity 


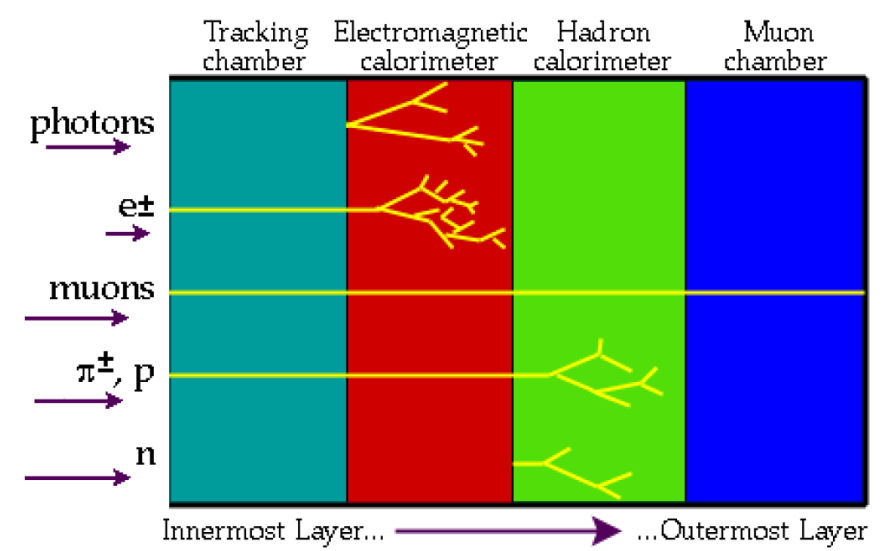

Figure 3.4: A schematic of how particles will interact in the detector [49].

$$
y=1 / 2 \ln \left[\left(E+p_{z}\right) /\left(E-p_{z}\right)\right]
$$

in the limit that $\frac{m}{E} \geq 0$, where $E$ is the energy of a particle and $p_{z}$ is the momentum in the $z$ direction. The term transverse is taken to mean, transverse to the beam pipe. So for a quantity $\mathbf{W}$, the transverse component is $\mathbf{W}_{\mathbf{T}}=$ $\left(W_{x}, W_{y}, 0\right)$ and $W_{T}=\sqrt{W_{x}^{2}+W_{y}^{2}}$.

\subsubsection{Central Tracking System}

The innermost component of the detector, surrounding the beam pipe, is the Tracking System which maps the trajectories, the "tracks", of the charged particles that pass through it. The basic concept is that as the charged particles pass through the material of this system they deposit energy which can be recorded and used to pin down the location of the particle. Many thin layers are placed one after another so as a particle passes through multiple layers, a map of the particle's path can be made. The most common material is a scintillating fiber, that produces light as the particle interacts with it. The system is designed so the particles passing through it, only have a minimal interaction with the material of 
the tracker i.e. they are not deflected or lose significant energy, hence their location can be determined without affecting the course they will take. The tracking system at $\mathrm{D} \varnothing$ is positioned in a solenoidal magnetic field, with field strength, $\mathbf{B}$, with the field lines parallel to the beam. This bends the tracks of the particles, enabling a measurement of the particle's momentum from the curvature of the track, $\rho$, and from the direction of the curvature, whether if it is positivity or negatively charged, see Equation 3.3.

$$
p_{T}=\rho|q \mathbf{B}|
$$

If multiple charged particles are produced in a specific interaction then there tracks can be traced back to the origin and the vertex of the interaction can be determined. DØ can locate a vertex to within $25 \mu \mathrm{m}$ along the beam axis and within $15 \mu \mathrm{m}$ in the transverse direction. This resolution results in decent lepton transverse momentum, $p_{T}^{\ell}$, jet transverse energy, $E_{T}^{j e t}$, and missing transverse energy, $\not_{\mathrm{T}}$ measurements. The $\mathbb{F}_{\mathrm{T}}$ is explained in more detail in Section 4.3.5.

Within the DØ detector there are two parts to the tracking system, a Silicon Microstrip Tracker, SMT, and a Central Fiber Tracker, CFT, along with the solenoid magnet. The layout of these systems can be seen in Figure 3.5.

\section{Silicon Microstrip Tracker}

The Silicon Microstrip Tracker, SMT, is a solid state tracker, using silicon as the interaction material. The silicon is normally kept under sufficient voltage to deplete the conduction region, but when a charged particle passes through, electrons are excited into the conduction region producing a measurable signal. The band gap in silicon is of the order of $1 \mathrm{eV}$, therefore particles, like those of interest in the detector, with energies on the order of $\mathrm{GeV}$, lose a negligible amount of energy. The SMT provides tracking and vertexing over nearly the 


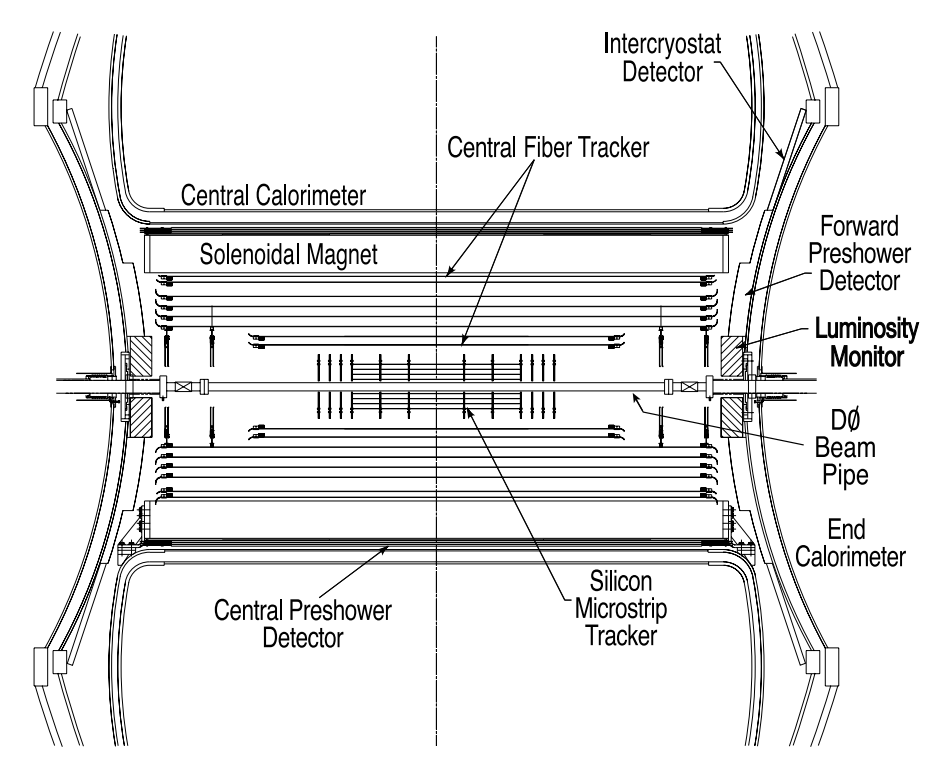

Figure 3.5: Diagrammatic representation the inner tracking system in the $\mathrm{x}-\mathrm{z}$ plane [48].

entire $\eta$ range at the $\mathrm{D} \varnothing$ detector (to $|\eta|<3$ ) and of the two trackers is the better at locating vertices. A diagrammatic representation of the SMT system is shown in Figure 3.6. The central region of the SMT has barrel modules, which measure in the $r-\phi$ plane, and are interspaced with disks which measure in $r-z$ and $r-\phi$ planes. The forward regions of the SMT has assemblies of disks. Therefore regions of the detector at high $\eta$ are measured by disks and small $\eta$ by barrels and the CFT. There are 6 barrels in the central region, each of which is capped at high $z$ with disks of 12 double-sided wedge detectors called an "F disk". In the far forward region there are two large disks called "H disks" for tracking at high $\eta$.

\section{Central Fiber Tracker}

Surrounding the SMT is the Central Fiber Tracker, CFT, a scintillating fiber tracker. Of the two trackers it has better transverse momentum resolution due to its larger radial coverage. It is located at $r=20$ to $52 \mathrm{~cm}$ from the beam pipe. 


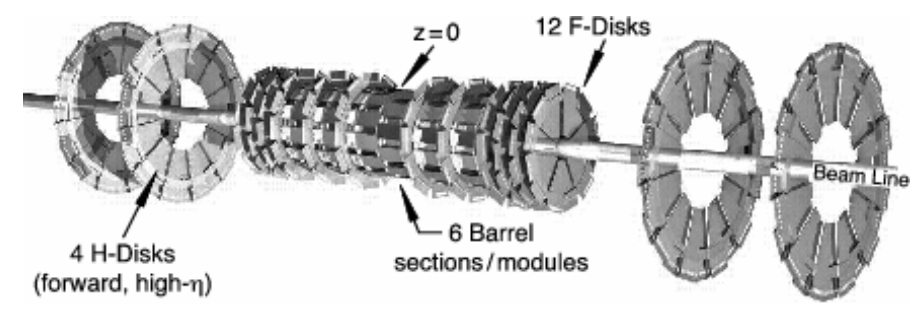

Figure 3.6: Diagrammatic representation the SMT [48].

The CFT has eight concentric cylinders of scintillators separated into two layers, an axial doublet layer and a stereo doublet layer [48]. The output from the axial layers can be read by the electronics during the interval of a bunch crossing time unlike the SMT, making it an important part of the Level One D $\varnothing$ trigger system (see Section 4.1).

\section{Solenoidal magnet}

The $\mathrm{D} \varnothing$ detector has a solenoidal magnet of $2.73 \mathrm{~m}$ length and $1.42 \mathrm{~m}$ in diameter. The size, as it was added after Run I, was determined by the available space. This magnet produces a $2 \mathrm{~T}$ field, designed to be as uniform over as large a percentage of the detector as possible, see Figure 3.7. As it has been explained previously having the tracking system of the detector inside a magnetic field allows one to determine the transverse momentum of particle. The location of the magnet inside the detector, is shown in Figure 3.8.

\subsubsection{Preshower Detectors}

Between the CFT and the calorimeters are the Preshower Detectors. These aid in electron identification and in the background rejection both for triggering and for offline reconstruction. The preshower detectors function both as calorimeters 


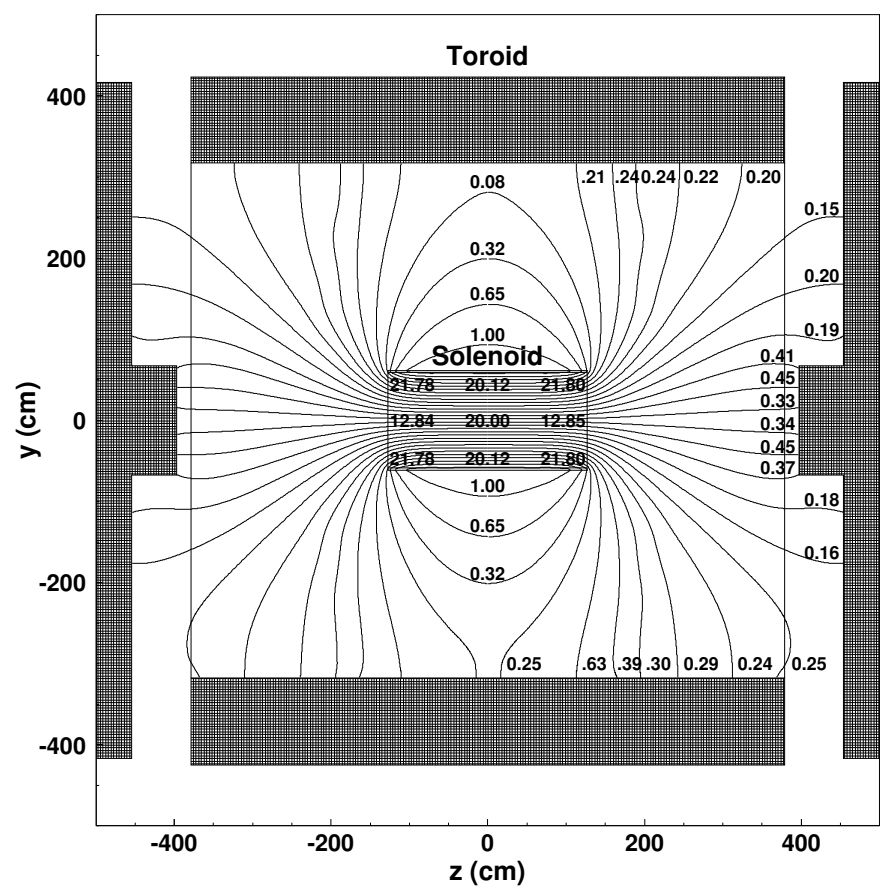

Figure 3.7: Diagrammatic representation of both the solenoidal and toroidal magnetic fields with in the $\mathrm{D} \varnothing$ detector [48].

and tracking detectors, hence improving the matching between tracks and showers. They are made of scintillating fibers, with layers of lead. The scintillator acts as a tracker giving momentum and positional information and the lead acts as a "calorimeter". The preshower detectors are in two segments; the Central Preshower Detector, CPD, which covers up to an $|\eta|$ of 1.3 and the Forward Preshower Detectors, FPD, which cover from an $|\eta|$ of 1.5 to 2.5.

\subsubsection{Electromagnetic and Hadronic Calorimeters}

The concept of a calorimeter is a system that will measure the energy of the particles produced. This is achieved by having a large dense material that the particles will deposit all of their remaining energy in, as they traverse through it. The energy will be deposited over a distance dependent on the interactions 


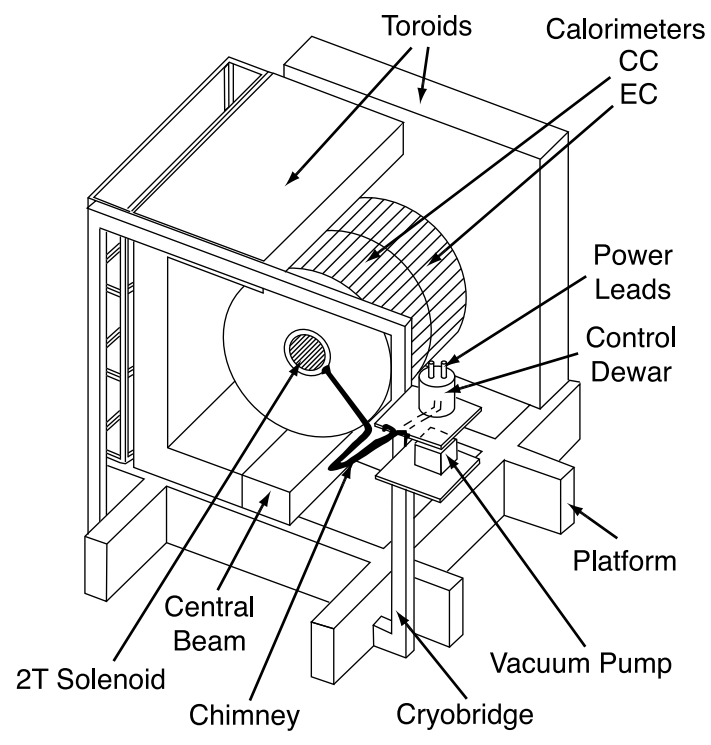

Figure 3.8: Diagrammatic representation of the position of both the solenoidal and toroidal magnets with in the $\mathrm{D} \varnothing$ detector [48].

undergone in the material and the initial energy of the particle. The calorimeters record energy deposited by electromagnetic interactions through pair-production and bremsstrahlung as well as that deposited by hadronic interactions. They are consequently separated into two types, an electromagnetic calorimeter and a hadronic calorimeter.

The size of an electromagnetic shower can be defined as being of a typical size know as a radiation length, $X_{0}$, and for a hadronic shower as the interaction length, $\lambda_{I}$. Radiation lengths are, for the same energy incident particle, shorter than interaction lengths, as EM reacting particles deposit their energy over smaller distances. Therefore EM calorimeters are located before hadronic calorimeters. The EM calorimeter has layers of thickness $20.5 X_{0}$ at $\eta=0$ which ensures the majority of a energy deposited by a particle undergoing EM showering will be in the EM calorimeter.

The hadronic calorimeter is further separated into Coarse and Fine sections. Both calorimeters are classified into two regions; the Central Calorimeter, CC, 


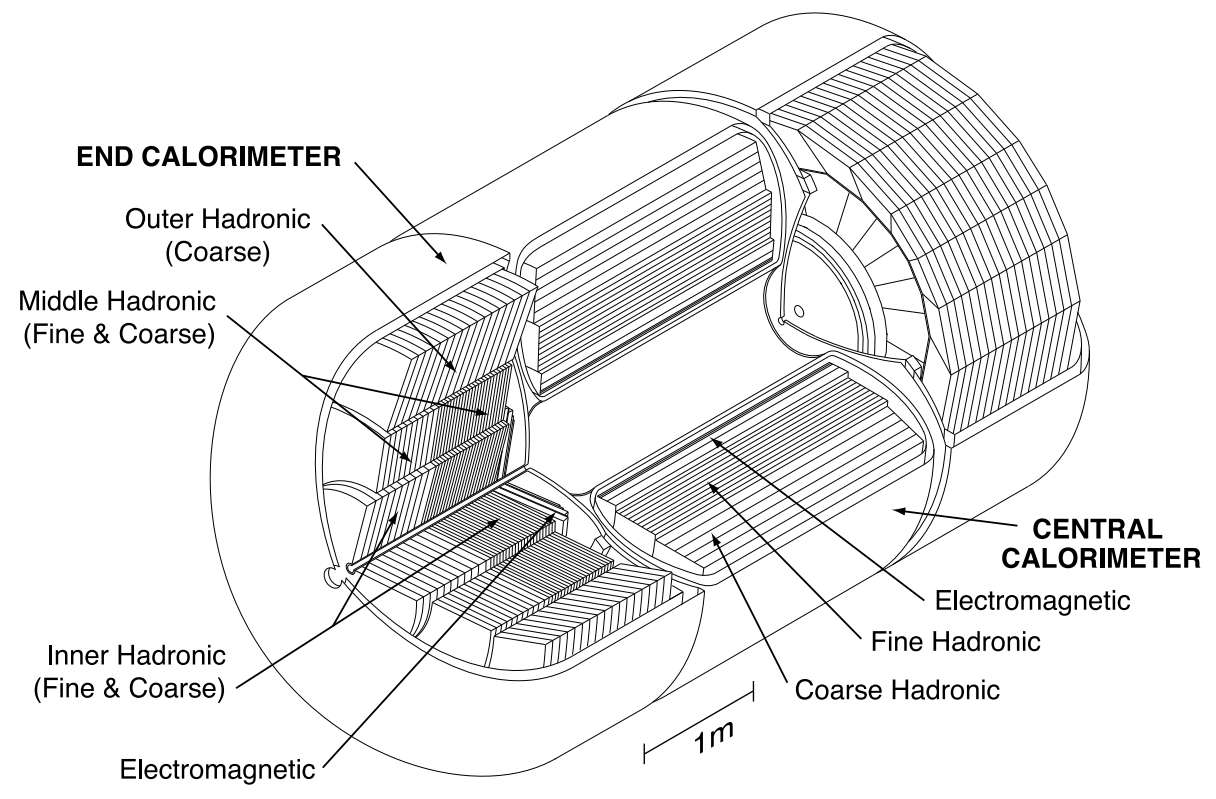

Figure 3.9: The layout of the Central Calorimeter, CC and End Calorimeter, EC, regions of the EM and hadronic calorimeters [48], both the Coarse and Fine sections of the hadronic calorimeters.

region covers values of $|\eta|<1$, and the north and a south End Calorimeter, EC, regions which covers values of $|\eta|$ from 1 up to about 4 . The $\mathrm{CC}$ and $\mathrm{EC}$ have the EM section of the calorimeter closest to the interaction region and are surrounded by first the fine and then coarse hadronic calorimeters. The layout of the $\mathrm{CC}$ and EC regions of the EM and hadronic calorimeters are shown in Figure 3.9.

The type of calorimeter at D $\varnothing$ is known as a "sampling" calorimeter, this means there are alternating layers of a "passive" medium of a dense absorber, followed by an "active" medium that produces a readable signal from which the deposited energy can be determined. For the DØ calorimeter, liquid argon is used as the "active" medium, placed in gaps between grounded "passive" absorber plates. When a shower begins in the plate the produced particles ionize the liquid argon and the electrons drift to the readout plate, held at a positive potential, with the size of the current proportional to the initial energy deposited. Each instance of this system is know as a cell and the layout of such a cell is shown in 


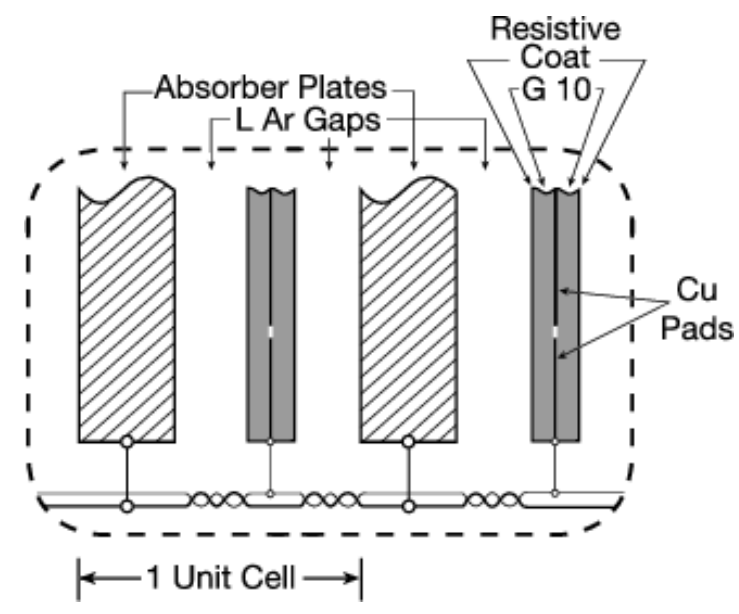

Figure 3.10: A diagram of a calorimeter cell showing the "passive" plates, the "active" gaps of liquid argon, and the copper readout pads [48].

Figure 3.10. Summing up the signal from all cells with deposited energy one can determine the total energy deposited in the calorimeter, see Section 4.2 for details on calorimeter energy reconstruction. For the EM calorimeter the absorber plates are nearly pure depleted uranium, with a width of $3 \mathrm{~mm}$ in the $\mathrm{CC}$ region and $4 \mathrm{~mm}$ in the EC region. For the fine hadronic calorimeter the absorber plates are uranium niobium alloy, of a width of $6 \mathrm{~mm}$ for both the $\mathrm{CC}$ and $\mathrm{EC}$ regions. For the coarse hadronic calorimeter, copper absorber plates are used in CC region and stainless steel plates in EC region.

The calorimeters have their own cryostat which cools them to a constant $90 \mathrm{~K}$ and is placed between the $\mathrm{CC}$ and $\mathrm{EC}$ calorimeter, resulting in poor coverage in this inter-cryostat region, ICR, region. To compensate for this poor energy resolution an extra active layer called the inter-cryostat detector, ICD, is placed in this region. It consists of 16 scintillating tiles designed to match the granularity of the EC calorimeter. Figure 3.11 shows the layout of the calorimeter including the ICR. The shading pattern shows groups of cells that use a single readout [48]. 


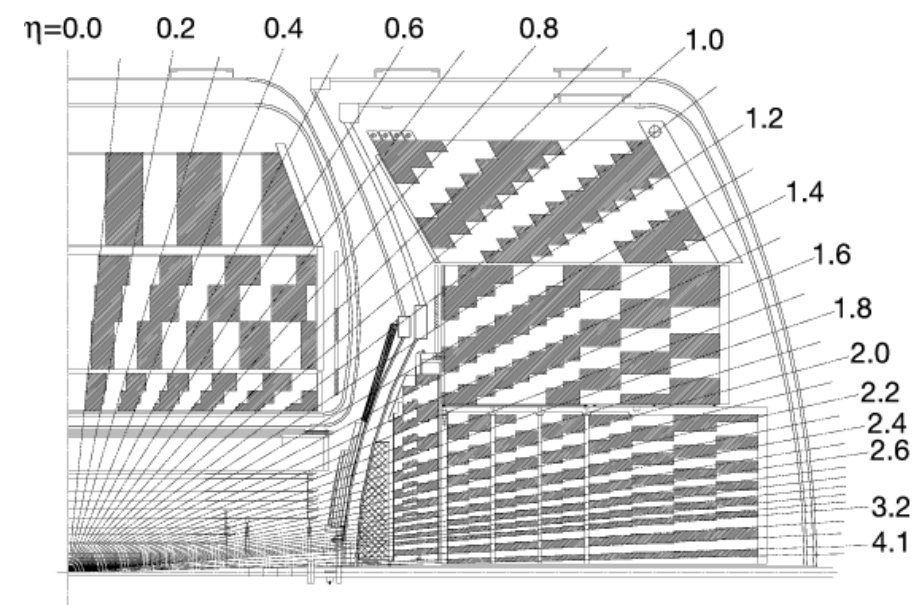

Figure 3.11: Diagrammatic representation of the layout of the cells with in the calorimeters, showing both the central and end calorimeters and the ICR [48].

\subsubsection{Muon System}

The outermost part of the detector is the Muon System. The muon's high mass, relative to that of the electron, results in them undergoing basically no bremsstrahlung interactions and they do not interact hadronically, hence the muon traverses through the rest of the detector without losing much energy. This is very unlikely for all other known particles so it is assumed that all particles seen in the Muon System are muons. Neutrinos will also pass through the detector unmeasured, but due to their very low interaction rate they are treated as missing energy. The Muon System has its own trackers and a dedicated toroid magnet of $1.9 \mathrm{~T}$, so there is an independent measurement of the $p_{T}$ and charge of the muon. The muon tracks are measured by proportional drift tubes, PDT, and there are additional scintillation counters to measure the muon's energy. The PDT's are a fast simple system used to determine a particle's position, where a wire is held in the centre of a tube of gas at a positive potential. A muon entering the tube ionizes the gas and releases electrons which drift to the wire. There are 

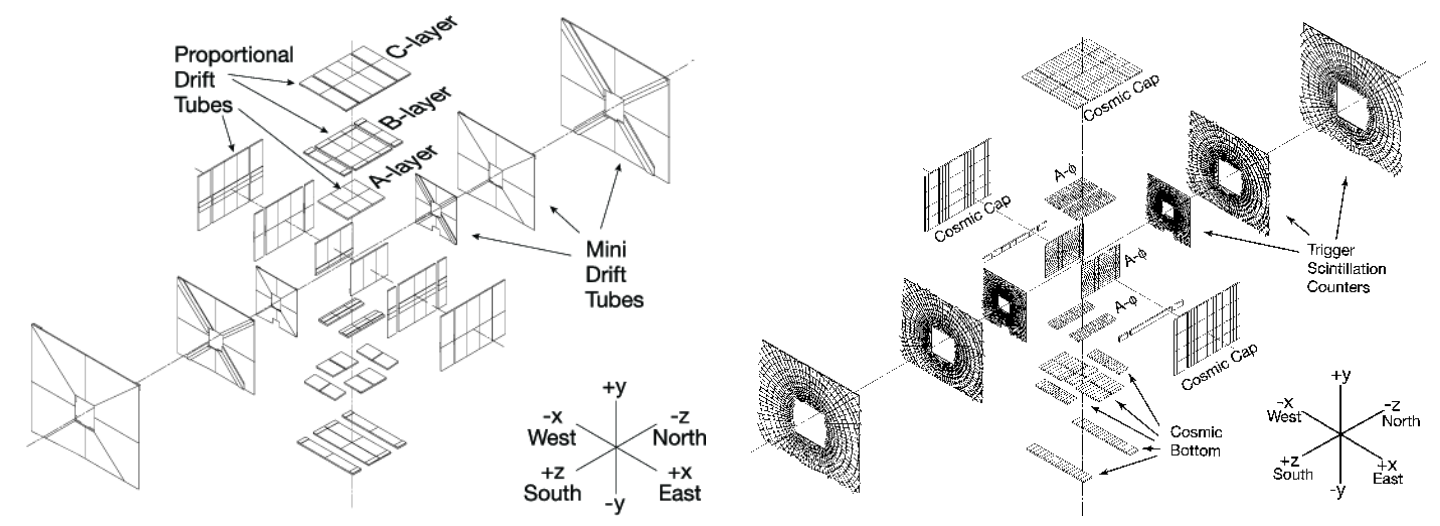

Figure 3.12: Diagrammatic representation of the PDT's (left) and the scintillation counters (right) for the muon system [48].

three layers to the PDT, A, B and C, an exploded view of the PDT's is shown in Figure 3.12 (a) and the muon system's scintillation counters in Figure 3.12 (b). As the PDT's are a fast response system they can be used to provide information on muons to the Level One trigger system. The Muon System also has a scintillation counter cosmic cap which lets one associate a muon seen in the PDT with one seen in a bunch crossing and used this information to reduce the cosmic ray background.

\subsubsection{Luminosity Monitors}

The D $\varnothing$ detecter also has specialized Luminosity Monitors. These are positioned at $\mathrm{z}= \pm 140 \mathrm{~cm}$ next to the beampipe and are designed to measure the rate of inelastic collisions. The majority of collisions produced are "soft" interactions with no high $p_{T}$ objects of interest, but this information can be used as first input to the triggering system, giving the information that a proton anti-proton collision has taken place. 


\section{Chapter 4}

\section{Event reconstruction}

\subsection{Triggers}

In order to determine the hard scatter events of interest out of the numerous soft events produced at the Tevatron, a filtering system know as a "trigger" system is used. This is run continuously during data taking and each event is studied to see if it passes a set of criteria that would indicate that it is of interest, such as containing high- $p_{T}$ objects or large amounts of $\mathscr{E}_{\mathrm{T}}$, at which point it is then written to tape. Due to constraints on timing and bandwidth this triggering is done in stages, with the information from the fastest readout components being used first and information on reconstructed particles in later stages. Object reconstruction at $\mathrm{D} \varnothing$ is described in Section 4.2. The triggers are separated into different physics types that record whether specific objects of interest have been reconstructed.

At DØ there is a three stage trigger system. Level One (L1) takes a continuos readout of events, events that pass Level One are passed to Level Two (L2), which uses both direct information from hardware and basic reconstructed information. Events that pass Level Two are then passed to the final level, Level Three (L3), which uses a simple reconstruction of the whole event. Technically there is also 
a Level Zero, using the information from the Luminosity Monitor on whether a collision has occurred. The three stages of the triggering system, with their respective rates and processing times, are shown in Figure 4.1.

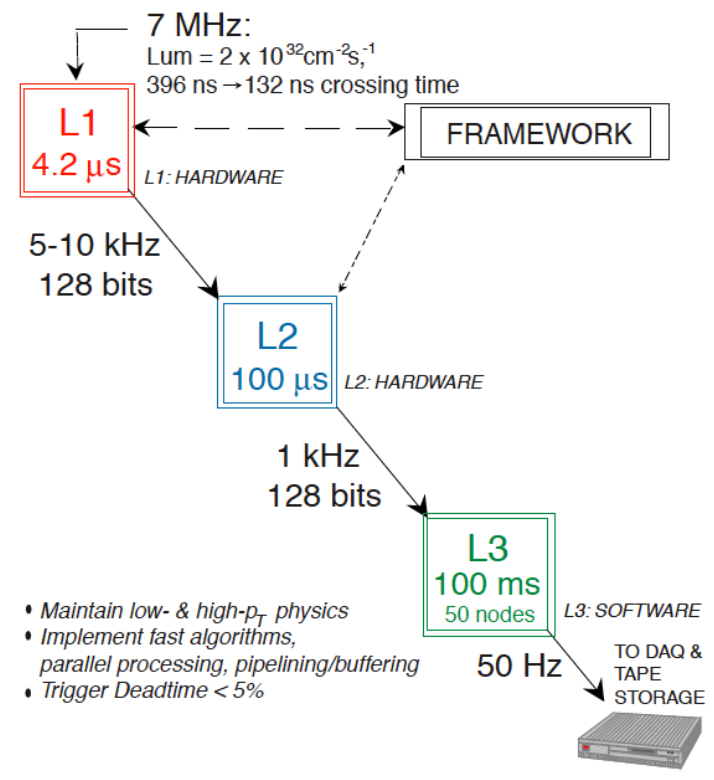

Figure 4.1: Diagrammatic representation of the triggering system, showing the three layers of the trigger system, L1, L2 and L3, and their associated rates and processing times [48]

\section{Level One}

The L1 trigger uses fast information provided by the CFT, both calorimeters, and the muon scintillation counters. The SMT readout is not fast enough to be considered by this trigger. The L1 trigger decides in less than $4.2 \mu \mathrm{s}$ if an event has passed one of the 128 predefined requirements [48] based on information it receives from these systems. This stage reduces the initial $7 \mathrm{MHz}$ peak rate to around $5 \mathrm{kHz}$. 


\section{Level Two}

The L2 trigger is a combination of both software and hardware triggers, in which information from both the detector level and basic reconstructed objects are used. Details on object reconstruction are given in Section 4.2. The L2 trigger makes a decision in $100 \mu \mathrm{s}$ and reduces the rate from the $5 \mathrm{kHz}$ maximum rate in the L1 trigger to $1 \mathrm{kHz}$.

\section{Level Three}

For the L3 trigger a simple reconstruction of the whole event is used, with information from all parts of the detector. This part of the triggering system takes up to $50 \mathrm{~ms}$ and reduces the output rate to less than $50 \mathrm{~Hz}$. Events which pass L3 are recorded to tape.

The rates of the triggering levels over the period of a store, are shown in Figure 3.2, with L1 shown in black, L2 in blue, and L3 in red. The triggering system is designed so that over the length of a store the maximum rate of events the system is capable of handling is recorded and the available bandwidth is not exceeded. Therefore a "prescale" is used, this is set to a predefined level limiting

the total rate of events processed. Therefore as the luminosity reduces over the course of a store the trigger rates also reduce and the prescale level is changed to refect this. This is shown in Figure 3.2, where numbers 1-5 reflect the points where the prescale level was changed.

\subsection{Detector component based reconstruction}

Events that pass the three levels of the trigger system undergo full reconstruction. This is where the information which has been recorded in the different 
components of the $\mathrm{D} \varnothing$ detector is combined to determine what types of particles were produced and with what characteristics. This is first separated into three parts, track reconstruction, vertex reconstruction, and calorimeter reconstruction and then using this information whole objects can reconstructed. Object reconstruction is described in Section 4.3.

\subsubsection{Track reconstruction}

From track reconstruction, information regarding the particles angular distribution, charge, and momentum from the curvature in the magnetic field, can be determined. Track reconstruction involves both a pattern recognition algorithm and a track finding algorithm. The pattern recognition algorithm looks for neighbouring signals which occur within a specific layer and groups them into clusters. It then hypothesizes possible tracks by combining clusters that may belong to a specific track. An iterative track fitting algorithm then determines the most likely combination that gives the particle's actual track [50]. Once a track has been determined information on the $p_{T}$ and charge can be determined by fitted to the track. There are two tracking finding algorithms used, the Histogramming Track Finder and the Alternative Algorithm [51], tracks found by both algorithms are used.

\subsubsection{Vertex reconstruction}

Using the reconstructed tracks the vertex or mutual interaction point can be reconstructed. This enables the grouping of particles that were produced in an interaction together and if they were produced in the hard scatter, to be determined.

The reconstruction of the primary vertex is performed with the "adaptive primary vertex algorithm" [52]. This looks for groupings of tracks and then 
performs a fit to determine if they originate from the same point. This is achieved by determining the $\chi^{2}$ of the tracks and then removing the track which contributes the most. This step is then repeated until some predefined $\chi^{2}$ is achieved and there are at least two tracks remaining [52]. The vertex matched tracks are then removed and this is repeated on the remaining tracks until the algorithm cannot reconstruct any more vertices.

The vertex due to the hard scatter is referred to as the primary vertex. As there can be multiple collisions per beam crossing, multiple primary vertices are possible but most will originate from low $p_{T}$ soft scatters. The high $p_{T}$ vertex from the hard scatter is considered the Primary Vertex, PV. To determine which is the PV, the probability that the vertex arises from a soft scatter ("Minimum Bias Probability"), is calculated from the distribution of the $\log \left(p_{T}\right)$ of the tracks assigned to the vertex. The vertex with the lowest value of the minimum bias probability is taken as the PV [53]. PV are also required to be formed from at least 3 tracks and that its distance from the $z$-axis, $\left|z_{p v}\right|<50 \mathrm{~cm}$. Secondary vertices which correspond to the decays of the daughters of the particles produced in the hard scatter, which can be identified by being displaced from the PV, are also identified.

\subsubsection{Calorimeter cell and cluster reconstruction}

The energy reconstructed from the active calorimeter cells provides information both on the energy of the particles produced and on the existence of neutral particles which would not have be seen in the central tracking system. When an object passes through the calorimeter it deposits energy in multiple calorimeter cells, which must be combined into a cluster which represents the total deposited energy of the object present. As many particles may be in the detector at one time, a clustering algorithm is used to sum together the energy in adjacent cells 
that corresponds to one particle's passing.

- Cell selection: Cells in the calorimeter suffer from noise both from electronics and from decay of the uranium in the detector [53]. Therefore the energy in a cell is used only if it is above a threshold known as "online zero suppression". The T42 [54] algorithm is used to identify which cells pass this threshold while retaining their neighbouring cells with a lower energy than this threshold. From the PV and the position of a cell, one can infer a direction associated with the energy in a cell which is used to compute the transverse energy, assuming a massless object.

- Cluster reconstruction: From multiple layers of the calorimeter that have deposited energy or "hits" in them at the same angle, a "tower" is defined. This is, many layers of cells with hits in them at the same position in $\eta$ and $\phi$. Towers with a $p_{T}$ greater than $500 \mathrm{MeV}$ are added to neighbouring towers, which have a $p_{T}>50 \mathrm{MeV}$ and are in a cone of size $\Delta R=\sqrt{\Delta \eta^{2}+\Delta \phi^{2}}<0.3$ in the $\mathrm{CC}$ region, or in a cone of $10 \mathrm{~cm}$ in the third layer of the EC region. If the total energy of these clusters is greater than $1 \mathrm{GeV}$ they are retained and all towers in $\Delta R<0.4$ are added [53]. The central position of the cluster is defined by the energy weighted mean of its cells in the third layer of the EM calorimeter [53].

If a match is found in the preshower detector then this additional information is used to produce an updated energy measurement which includes the energy from this subdetector. The preshower information is also used to update the positional information. 


\subsection{Object reconstruction}

The information from the particle's tracks and the energy clusters reconstructed in the detector can be used to reconstruct whole objects and with them the whole event. This section will describe in detail the reconstruction of the particles relevant to the analyses covered in this thesis.

\subsubsection{Muon reconstruction}

Muons are minimally ionizing particles, therefore they are expected to travel through the detector with minimal interactions. The $\mathrm{D} \varnothing$ detector can identify muons using three independent subsystems $[55,56]$ :

- Local muons: Muons are classified as "local" if they are identified in the muon detector system. The muon system, as shown in Figure 3.12 consists of three layers, A, B, and C and covers $90 \%$ of the angular acceptance of the detector, up to $|\eta|=2$. Local muons can have a momentum measurement if a track segment is reconstructed in this subsystem.

- Central track matched muons: Muons that are reconstructed in the central tracking detectors. The central trackers are efficient at detecting muons over all the of angular acceptance of the detector and muons detected here will the lowest momentum resolution.

- Muon tracking in the calorimeter, MTC: Muons can also be identified with a low efficiency $(\approx 50 \%)$ in the calorimeter, by identifying particles with the signature of a minimally ionizing particle.

Muons are defined as being of a specific "quality", based on the reconstruction criteria that the muon passes. Muons are classified in terms of isolation quality, track quality, and identification quality in the muon system. The efficiencies are 
determined by the tag and probe method [57] using a sample of $Z / \gamma^{*} \rightarrow \mu \mu$ events for both data and MC simulations [55]. The quality requirements relevant to the analyses preformed in this thesis will be described.

\section{Muon identification quality in the muon system}

The muon identification quality in the muon system is classified based on the value Nseg. Positive values correspond to a local muon with a central matched track and negative values to local muon that do not have a central matched track. The value of $\mid$ Nseg $\mid(0,1,2$ or 3$)$ corresponds to the different segments in the PDT system that have muon hits. The signals in the detector that correspond to different classifications of Nseg are shown in Table 4.1. The additional requirements on a muon for it to pass the loose and medium criteria depend on the value of $\mid$ Nseg| [55, 56].

- For $\mid$ Nseg $\mid=3$ a muon is defined as medium if it fulfills all the following criteria. It is defined as loose if three out of the following four are fulfilled, with at least one scintillator hit always being required.

$-\geq 2$ layer A layer wire hits

$-\geq 1$ layer A layer scintillator hit

$-\geq 2$ layer $\mathrm{B}$ or $\mathrm{C}$ layer wire hits

$-\geq 1 \mathrm{~B}$ or $\mathrm{C}$ layer scintillator hit

All muons with $|\mathrm{Nseg}|<3$ must be matched to a track in the central tracking system.

- $|\mathrm{Nseg}|=2$, is classified as medium if it has a BC segment matched to a central track. For a loose muon it must fulfill one of the following two requirements. 


\begin{tabular}{|c|c|c|c|}
\hline Nseg & Muon track & $\begin{array}{c}\text { Central track matching } \\
\text { algorithm }\end{array}$ & $\begin{array}{l}\text { MTC matching } \\
\text { criteria }\end{array}$ \\
\hline 3 & $\begin{array}{l}\text { Central track and } \\
\text { local muon track } \\
\text { (A and BC layer) }\end{array}$ & $\begin{array}{l}\text { Muon to central if local } \\
\text { muon track fit converged } \\
\text { Central to muon otherwise }\end{array}$ & $\begin{array}{c}\Delta \eta, \Delta \phi \text { between MTC } \\
\text { and central track } \\
\text { extrapolated to cal. }\end{array}$ \\
\hline 2 & $\begin{array}{l}\text { Central track } \\
\text { and BC layer }\end{array}$ & Central to muon & As above \\
\hline 1 & $\begin{array}{l}\text { Central track } \\
\text { and A only }\end{array}$ & Central to muon & As above \\
\hline 0 & $\begin{array}{l}\text { Central track and } \\
\text { muon hit or central } \\
\text { track and MTC }\end{array}$ & $\begin{array}{l}\text { Central to muon } \\
\text { central to cal. }\end{array}$ & As above \\
\hline-1 & A segment only & No match & $\begin{array}{c}\Delta \eta, \Delta \phi \text { between MTC } \\
\text { and A-layer } \\
\text { segment }\end{array}$ \\
\hline-2 & BC segment only & No match & $\begin{array}{c}\Delta \eta, \Delta \phi \text { between MTC } \\
\text { and BC-layer } \\
\text { segment }\end{array}$ \\
\hline-3 & $\begin{array}{l}\text { Local muon track } \\
\qquad(\mathrm{A}+\mathrm{BC})\end{array}$ & No match & $\begin{array}{c}\Delta \eta, \Delta \phi \text { between MTC } \\
\text { ocal muon track at } \\
\text { A-layer it fit converged } \\
\text { or else A-segment position }\end{array}$ \\
\hline
\end{tabular}

Table 4.1: The muon requirements for the different values of Nseg [55]. Calorimeter is abbreviated to Cal. Showing the muon tracks hits, the algorithm used for track matching and the matching to the calorimeter muons, for each value of Nseg.

$-\geq 1 \mathrm{~B}$ or $\mathrm{C}$ layer scintillator hit

$-\geq 1 \mathrm{~B}$ or $\mathrm{C}$ layer wire hit

- $|\mathrm{Nseg}|=1$ muon is defined as loose if it satisfies the following.

$-\geq 1$ scintillator hit

$-\geq 2$ layer A wire hits

for a $\mid$ Nseg $\mid=1$ muon to be defined as medium it must also have a probability to reach the $\mathrm{BC}$ layer of less than 0.7 , i.e. it must be in the bottom 
part of the detector $(\eta<1.6)$ or have low momentum [55] [56].

\section{Track quality}

At $\mathrm{D} \varnothing$ the track quality requirements are based on three things $[55,56]$, the number of hits in either the SMT or CFT, the $\chi^{2}$ per degree of freedom of the central track fit, and the distance of closest approach, dca, in $(x, y)$, with respect to the beam line. For the track quality definitions relevant to the analysis performed in this thesis the following is required:

\section{TrackLoose:}

- The distance of closest approach $\mid$ dca $\mid<0.2 \mathrm{~cm}$ or if there is a SMT hit then $\mid$ dca $\mid<0.02 \mathrm{~cm}$.

\section{TrackMedium:}

- The distance of closest approach $\mid$ dca $\mid<0.2 \mathrm{~cm}$ or if there is a SMT hit then $\mid$ dca $\mid<0.02 \mathrm{~cm}$.

- The $\chi^{2}$ per degree of freedom is less than 4 .

\section{Isolation quality}

This quality criteria requires that the muon be isolated, i.e. that it does not have any reconstructed tracks or calorimeter energy clusters near it. It is required that the sum of the $E_{T}$ in the calorimeter cells, in a cone of size $\Delta R$ around the cluster, is less than a predefined value and that the sum of the $p_{T}$ of the tracks, in a cone of $\Delta R$ around the track, is less than a predefined value. These values are given for the isolation requirements used in the analyses covered in this thesis $[55,56]$.

\section{NP Tight:}


- The sum of the transverse energy in the calorimeter cells in an annulus of size $0.1<\Delta R<0.4$ is less than $2.5 \mathrm{GeV}$.

- The sum of the transverse momentum of the tracks in a cone of size $0.0<$ $\Delta R<0.5$ is less than $2.5 \mathrm{GeV}$.

\section{TopScaledLoose:}

- The sum of the transverse energy in the calorimeter cells in a annulus of size $0.1<\Delta R<0.4$, scaled by the $p_{T}$ of the muon is less than 0.2 .

- The sum of the transverse momentum of the tracks in a cone of size $0.0<$ $\Delta R<0.5$, scaled by the $p_{T}$ of the muon is less than 0.2 .

Using these values scaled by the $p_{T}$ of the muon helps to reject more muons at low $p_{T}$. Low $p_{T}$ muons are more likely to be from secondary interactions within the detector and hence are less likely to be of interest [55].

\subsubsection{Tau lepton reconstruction}

Tau leptons decay in the detector into both leptons and hadrons, specifically $18 \%$ of tau lepton decays are to electrons, $17 \%$ to muons and the rest of the decays are to hadrons [23], see Figure 4.2. At DØ, when the tau lepton decays into electrons or muons these leptons are reconstructed as described in Sections 4.3.1 and 4.3.3 for electrons and muons, respectively. For the decays of tau leptons to hadrons, the main decays are to charged and neutral pions. To reconstruct these hadronic tau lepton decays both track and energy reconstruction is needed.

\section{Reconstruction algorithm}

The tau lepton reconstruction algorithm can be separated into two parts [59], one based on the calorimeter reconstruction and one on the track reconstruction. 

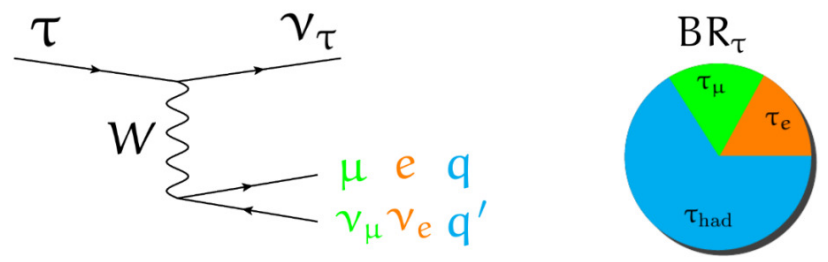

Figure 4.2: The percentage of the decay of the tau leptons into hadrons and leptons [58] and the Feynman diagram of a tau lepton decay to leptons and quarks.

- Calorimeter reconstruction: In the calorimeter tau leptons are identified by a calorimeter cluster using the Simple Cone Algorithm [60], with a cone of size $\Delta R=0.3$ and an isolation cone of $\Delta R=0.5$ [59]. They are also identified by EM subclusters which originate from individual $\pi^{0}$ decays. The EM subclusters are identified in the third layer of the EM calorimeter, by the Nearest Neighbour Algorithm. If such a subcluster is found then hits from the other layers of the EM calorimeter and the preshower detectors are added.

- Track reconstruction: All hadronic tau decay modes have between one and three associated tracks. Other tracks in the event will originate from the underlying event or misreconstruction of tracks. These additional tracks are a powerful discrimination tool, as the scalar sum of the $p_{T}$ of these tracks is generally small for isolated tau leptons and large from tracks arising from quark or gluon jets [59]. In reconstructing the tracks from tau leptons, all tracks with a $p_{T}>1.5 \mathrm{GeV}$ in a cone of size $\Delta R=0.5$ around the centre of the calorimeter cluster are considered. The track with the highest $p_{T}$ will be associated with the cluster. If a track is identified, up to two additional tracks, with $\mid$ dca $\mid<2 \mathrm{~cm}$ are also considered. A second track is added if the combined mass of the tracks is $<1.1 \mathrm{GeV}$ and a third track if it is $<1.7$ 
GeV.

When referring to the momentum of the tau lepton candidates, the momentum as reconstructed in the calorimeter is used, $p_{T}$, and the momentum as reconstructed from the tau lepton tracks will be specified specifically, $p_{T}^{\text {track }}$.

\section{Tau lepton Types}

Three tau lepton "Types" are defined which correspond to the main hadronic decay types. These are based on the number of the tracks and if there is a reconstructed cluster or EM subcluster. These three types are shown in Table 4.2, along with the decays they correspond too, the branching ratio to those decays and the required detector signature for each type.

\begin{tabular}{|c|c|c|c|}
\hline$\tau$ lepton Type & Physical Process & $\mathrm{BR}_{\tau}$ & Detector Signature \\
\hline 1 & 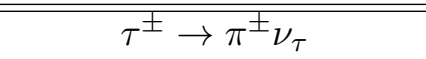 & $10.9 \%$ & 1 calorimeter cluster, 1 track \\
\hline 2 & $\begin{aligned} \tau^{ \pm} & \rightarrow \rho^{ \pm}\left(\rightarrow \pi^{0} \pi^{ \pm}\right) \nu_{\tau} \\
\tau^{ \pm} & \rightarrow\left(\geq 2 \pi^{0}\right) \pi^{ \pm} \nu_{\tau}\end{aligned}$ & $36.5 \%$ & $\begin{array}{c}1 \text { calorimeter cluster, } 1 \text { or more } \\
\text { EM sub-cluster, } 1 \text { track }\end{array}$ \\
\hline 3 & $\tau^{ \pm} \rightarrow \alpha_{1}^{ \pm}\left(\rightarrow \pi^{ \pm} \pi^{\mp} \pi^{ \pm}\right) \nu_{\tau}$ & $13.9 \%$ & $\begin{array}{c}1 \text { calorimeter cluster, } 1 \text { or } \\
1 \text { or more track, } \\
\text { with or without EM subcluster }\end{array}$ \\
\hline
\end{tabular}

Table 4.2: The three defined types of tau leptons, showing the decays they correspond to and the signature of them in the detector and the branching ratio of those decays [59]. Where $\mathrm{BR}_{\tau}$ is the percentage of tau leptons decaying to each tau lepton type.

\section{Tau lepton Neural Network}

The objects that are reconstructed by the algorithms described in Section 4.3.2 contain a large portion of jets that are misreconstructed as tau lepton events, especially $W+$ jet and multijet events [61]. Therefore to separate the hadronically decaying tau leptons from the large jet background, a multivariate analysis technique is used, specifically a Neural Network, $N N_{\tau}$. For more detailed information 

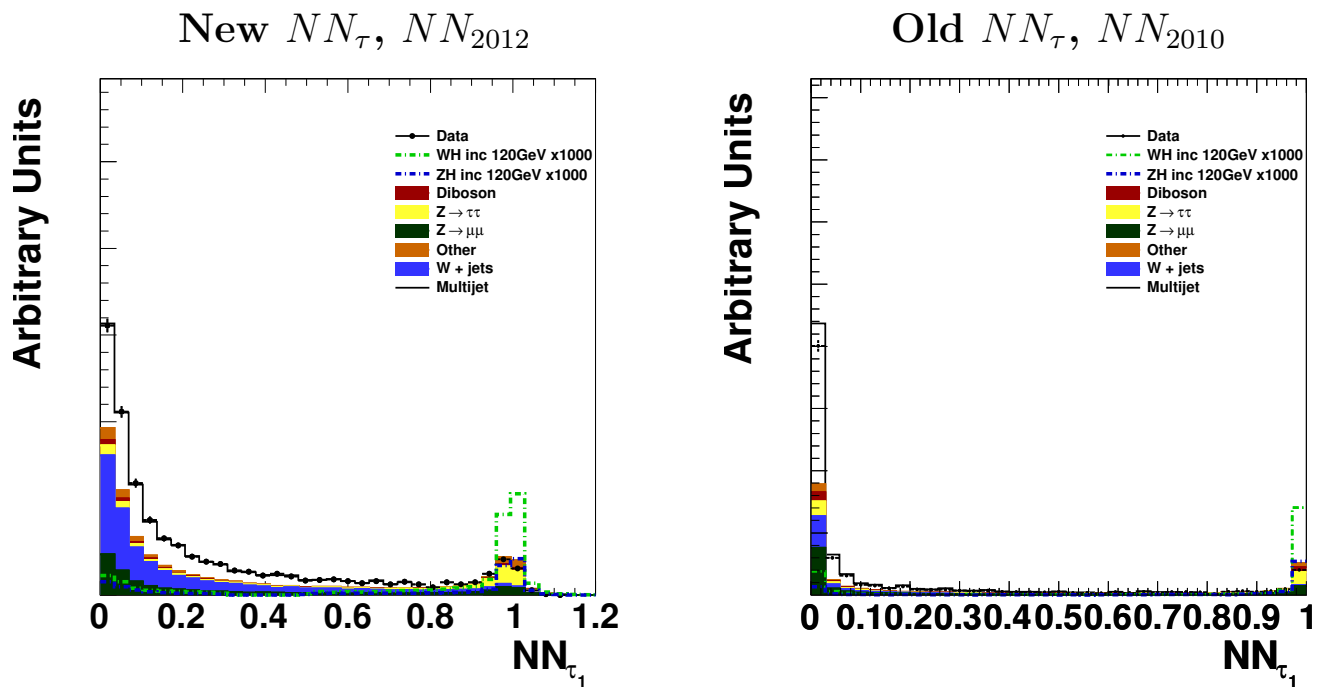

Figure 4.3: A comparison of the shape differences of the $N N_{2010}$ (old) and the $N N_{2012}$ (new) $N N_{\tau} \mathrm{s}$.

on multivariate analysis techniques, see Section 7.9.

The premise is that hadronically decaying tau leptons will have observables which look different from jets that are misreconstructed as tau leptons, with tau leptons producing low multiplicity narrow jets. Therefore training a $N N_{\tau}$ on a sample of tau leptons against a sample of jets misreconstructed as tau leptons one can construct a variable with a large discriminating power between them, enabling the removal of the majority of the misreconstructed tau leptons. The $N N_{\tau}$ is trained separately for each of the three types. For the analyses covered in this thesis two different $N N_{\tau}$ are used, referred to separately as $N N_{2010}$ and $N N_{2012}$, and shown in Figure 4.3.

To train and test the $N N_{\tau} \mathrm{s}$ two different samples are used for both, the signal sample (containing tau leptons), and the background sample (containing misreconstructed tau leptons). For the signal sample, a training sample of $Z / \gamma^{*} \rightarrow \tau \tau$ as produced by the PYTHIA MC generator [62] is used and for testing $Z / \gamma^{*} \rightarrow \tau \tau$ from the Alpgen MC generator plus Pythia are used [63], (see Chapter 5 for 
detailed information about $\mathrm{MC}$ generators). The two $N N_{\tau} \mathrm{s}$ are trained against $W+$ jet Alpgen plus Pythia MC and a multijet sample is used to test the $N N_{\tau} \mathrm{S}[58,64]$. Either 10 or 12 variables are used to train the $N N_{\tau}$, depending on the tau type. The variables are [58] [64]:

- The isolation in the tracking system, Track Iso, defined as,

$$
\text { track Iso }=\frac{\sum_{\text {track }} p_{T}^{\text {track }}}{\sum_{\text {track }} p_{T}^{\tau_{\text {track }}}}
$$

- The isolation in the calorimeter, defined as the ratio $E_{T}$ around the tau lepton candidates,

$$
\text { Cal Iso }=\frac{E_{T}(0.3<\Delta R<0.5)}{E_{T}(\Delta R<0.3)}
$$

- The fraction of hadronic energy in the shower, $F_{\text {had }}$, where $E^{F H}$ is the fraction of energy in the fine hadronic calorimeter, $E^{C H}$ is the fraction of energy in the coarse hadronic calorimeter,

$$
F_{\text {had }}=\frac{E^{F H}+E^{C H}}{E}
$$

- The fraction of EM subcluster energy in the shower, $E_{E M}$

$$
F_{e m}=\frac{E_{T}^{E M}}{E_{T}}
$$

- The distribution of EM energy in the subclusters, $D_{E M}$. $E_{T}^{E M 3}$ is the energy in the third layer of the EM calorimeter,

$$
D_{E M}=\frac{E_{T}^{E M}}{E_{T}^{E M 3}(\Delta R<0.5)}
$$

- The shower size, the root mean square of the calorimeter cluster of the tau lepton candidates,

$$
R M S=\sqrt{\sum_{i} \frac{E_{T}^{i}}{E_{T}}\left(\Delta \phi_{i}^{2}+\Delta \eta_{i}^{2}\right)}
$$


- The transverse energy distribution in the leading two towers, $E_{T}^{1}, E_{T}^{2}$ over the total energy distribution,

$$
E_{T_{\text {tower }}}=\frac{E_{T}^{1}+E_{T}^{2}}{E_{T}}
$$

- The energy fraction at the start of the EM shower, EM start where only the EM energy in layer 1 or 2 is considered,

$$
E M_{\text {start }}=\frac{E^{E M 1}+E^{E M 2}}{E}
$$

- The neutral energy fraction, $E T_{\text {sum }}$

$$
E T_{\text {sum }}=\frac{E_{T}}{E_{T}+p_{T}}
$$

- The angular correlation between the EM calorimeter and the hadronic calorimeter,

$$
\delta \alpha=\frac{1}{\pi} \sqrt{\frac{\Delta \phi^{2}}{\sin \theta}+\Delta \eta^{2}}
$$

- The fraction of the leading $p_{T}$ track momentum, $p_{T}^{\text {leading }}$ to the $E_{T}$,

$$
F_{\text {leading }}=\frac{p_{T}^{\text {leading }}}{E_{T}}
$$

- The $\eta$ dependance, redefined to be in the range 0 to 1.

$$
\eta_{d}=-\frac{\ln (\tan (\theta / 2))}{3}
$$

All variables are used to train the $N N_{\tau}$, except for the case of Type-1 tau leptons when the fraction of the EM energy and the angular separation are not used. Improvements for the $N N_{2012}$ compared to $N N_{2010}$ include that two of the variables are changed, to make more physically meaningful variables [58]. For the angular separation in Equation 4.10 the $\sin \theta$ is removed and for Equation 4.5 the 
cone size is reduced from 0.5 to 0.3 . To further improve the performance of the $N N_{2012}$ over the $N N_{2010}$, the TMVA [65] training parameters were optimized and the tau leptons are run throught the DØb-tagging tools. For Type-1 and Type2 tau leptons only the Jet Lifetime Inpact Parameter, JLIP, was used, where JLIP is the product of the probabilities of each examined track originating at the PV. For Type-3 the full DØb-tagging Neural Network, $N N_{b}$ can be used. This requires information on the displaced vertex which is not aviable for Type-1 or Type 2 tau leptons as they only have one track [58].

The $N N_{\tau}$ response for the three tau lepton types is shown in Figure 4.4 and shows the improved response from the $N N_{2012}$. In general there is about a $10 \%$ improvement on the tau letpton selection efficiency between the $N N_{2012}$ and $N N_{2010}[58]$.

In the inner cryostat region of the calorimeter Type-2 tau leptons cannot be reconstructed due to the requirement on an EM subcluster. All the Type-2 tau leptons within this region will be reconstructed as Type-1 tau leptons. These misreconstructed Type-2 tau leptons will have a different signature to normal Type- 1 tau leptons and therefore tau leptons in this region are trained separately.

\subsubsection{Electron reconstruction}

Electron reconstruction in the DØ detector consists of an EM cluster in a cone of $\Delta R=0.2$ in the calorimeter, matched to high $p_{T}$ track in the central tracking systems. For certain $|\eta|$ regions confirmation can also come from the preshower detector, $|\eta|<1.1$, for the CPD, and $1.5<|\eta|<2.5$ for the FPD [66]. Electrons will deposit the majority of their energy in the first few layers of the EM calorimeter, and hence their EM fraction (Equation 4.13) will generally be large. They will also normally be isolated with little other energy around them in the calorimeter and their longitudinal and lateral shape will be as expected for an 

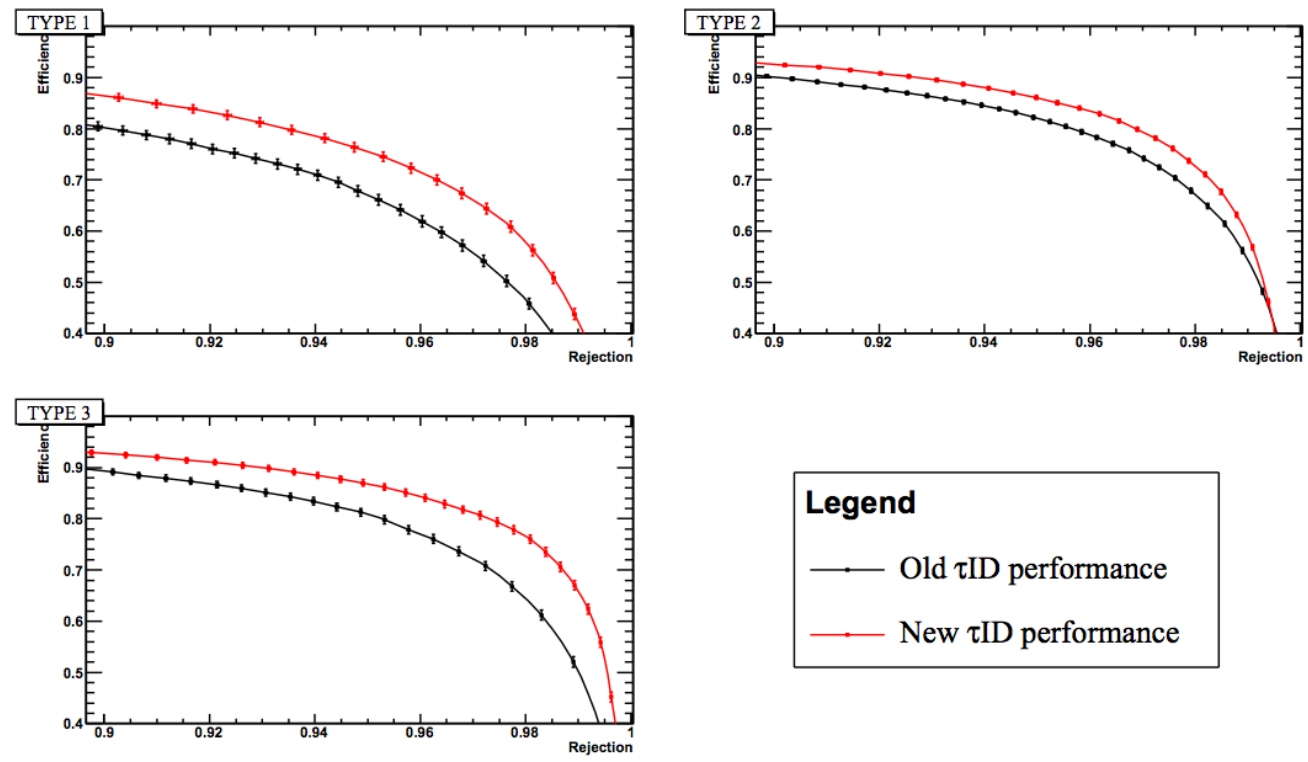

Figure 4.4: The efficiency compared to rejection of the $N N_{2012}$ in red (new) and the $N N_{2010}$ in black (old) [58]. This is shown for Type-1 (top-left), for Type-2 (top-right) and Type-3 (bottom-left).

electron, to utilize this information an algorithm, H-matrix, which compares the energy in each layer of the EM calorimeter with the total energy as predicted from simulations [66] [67] was created. Using this knowledge electrons are classified into a set of defined qualities, Point 0, Point 05, Point 1, and Point 2, as defined in Table 4.3. The requirements for the electron qualities depend on whether the electron is detected in the CC or EC regions. Electron identification is supported in the $|\eta|$ regions of less than 1.1 and between 1.5 and 3.2 [68]. This is due to the ICR region in the calorimeter where electrons are reconstructed poorly. The following properties are used to define the electron quality [68]:

- The EM fraction, EMf,

$$
\mathrm{EMf}=\frac{E_{E M}}{E_{t o t}}
$$

- The calorimeter isolation, the fraction of energy in the region between the 
isolation cone $(\Delta R=0.4)$ and the EM cluster cone $(\Delta R=0.2)$,

$$
\text { iso }=\frac{E_{\text {tot }}^{\Delta R=0.4}-E_{E M \text { Mluster }}^{\Delta R=0.2}}{E_{E \text { Ecluster }}^{\Delta R=0.2}} .
$$

- The calorimeter isolation after subtraction of expected zero bias interactions, isoE0, where zero bias events are explained in Section 5.2.2.

- The track isolation, IsoHC4, in a cone $0.4<\Delta R<0.5$ around the EM cluster,

$$
\mathrm{IsoHC4}=\sum E_{\text {track }} / E_{\text {EMcluster }}^{\Delta R=0.2}
$$

- The H-matrix, Hmx [67].

- The shower width of the EM cluster in the third layer, Sigphi.

- A Neural Net for electrons, $N N_{e}$, [69]. A multivariate analysis technique, trained using seven input variables, to select electrons from other objects that have been misreconstructed as such. More information on multivariate analysis techniques is available in Section 7.9.

- The track match probability, determined from the $\chi^{2}$ distribution, TrkMatch.

- The Hits on the Road, HoR. This uses the hits in the central tracking system to separate electrons from photons [70].

- The likelihood function can be used to further discriminate electrons from objects that have been misreconstructed as such. The likeihood function is trained using 8 input variables, Lhood8, [71].

- The ratio of EM cluster energy to the track matched momentum, $E / p$. 


\begin{tabular}{l|cccccccc}
\hline Variable & \multicolumn{2}{|c}{ Point 0 } & \multicolumn{2}{c}{ Point 1 } & \multicolumn{2}{c}{ Point 2 } & \multicolumn{2}{c}{ Point 05 } \\
& CC & EC & CC & EC & CC & EC & CC & EC \\
\hline \hline EMf & 0.09 & 0.90 & 0.90 & 0.90 & 0.09 & 0.90 & 0.09 & 0.90 \\
\hline IsoE0 & 0.09 & 0.1 & 0.08 & 0.1 & 0.08 & 0.06 & 0.15 & 0.05 \\
\hline IsoHC4 & 4.0 & (a) & 2.5 & $(\mathrm{a})$ & 2.5 & (a) & 3.5 & (b) \\
\hline Hmx & - & 40 & 35 & 40 & 35 & 40 & - & 10 \\
\hline Sigphi & - & (c) & - & $(\mathrm{c})$ & - & $(\mathrm{c})$ & - & (c) \\
\hline$N N_{e}$ & 0.4 & 0.05 & 0.9 & 0.05 & 0.9 & 0.1 & 0.3 & 0.2 \\
\hline TrkMatch & 0.0 & - & 0.0 & 0.0 & 0.0 & 0.0 & 0.0 & - \\
or HoR & 0.6 & - & - & - & - & - & - & - \\
\hline Lhood8 & - & - & 0.2 & 0.05 & 0.6 & 0.65 & 0.05 & - \\
\hline E/p & - & - & 8.0 & - & 3.0 & 6.0 & 8.0 & -
\end{tabular}

(a). IsoHC4 $<0.01$, or, IsoHC $4<(-2.5 \times|\eta|+7.0)$

(b). IsoHC4 $<0.01$, or, IsoHC4 $<(-2.5 \mathrm{x}|\eta|+5.0)$

(c). $|\eta|<=2.6$, SigPhi $>(6.5 /(-\eta \mid+5.0)-2.8)$

Table 4.3: The requirements on the electrons for the four different quality definitions for electrons [68] on the defined variables.

\subsubsection{Jet reconstruction}

Jets formed through hadronization interact in the calorimeters and produce showers of particles, which will deposit energy in numerous calorimeter cells. To reconstruct the jets a clustering algorithm that assigns calorimeter clusters and stable particles (those that do not decay in the detector) to a specific jet is used. There are several jet reconstruction algorithms used at D $\varnothing$, for the analysis discussed here the Run II Midpoint Cone algorithm [60] is used. To identify jets the centroid of a jet is defined to be $\left(y_{\text {jet }}, \phi_{\text {jet }}\right)$ and objects are defined to be in the jet cluster if they are in a cone of size $\Delta R=0.5$ relative to the jet centroid. All jets with a four momenta less than $6 \mathrm{GeV}$ are discarded [53]. Additional requirements based on the fraction of energy deposited in the EM and hadronic calorimeters, are used to remove jets that could be due to EM clusters or noisy cells in the calorimeter [53].

The measured energy of the jet, $E_{\text {jet }}^{\text {meas }}$ may not be a good measure of the final 
state particle jet, $E_{\text {jet }}^{\text {part }}$, due to energy not reconstructed in the cone, a nonlinear calorimeter response, additional energy in the cone due to noise in the calorimeter cells, or pile-up. To account for this a jet energy scale, JES, is applied, defined as,

$$
E_{j e t}^{\text {part }}=\frac{E_{\text {jet }}^{\text {meas }}-E_{O}}{R_{\text {jet }} S_{j e t}}
$$

where $E_{o}$ is the offset energy, due to noise in the detector, additional $p \bar{p}$ interactions or additional energy in the calorimeter from pile up. $R_{j e t}$ is the energy response of calorimeter to jets, this is generally much less than one, due to energy that has been deposited in the detector before the calorimeter and due to energy lost in the electronic systems between calorimeter modules. $S_{j e t}$ is a correction for the fraction of the jets energy that has been deposited outside the cone radius [53]. A second correction, jet shifting smearing and removal, JSSR, is used to correct the resolution of jets in $\mathrm{MC}$ events to what is seen in data.

\subsubsection{Missing Transverse Energy}

The final object to reconstruct from the detector information is the missing transverse energy, $\mathbb{F}_{\mathrm{T}}$. As the $\mathrm{D} \varnothing$ detector has areas not covered by some systems, due to internal supports or electronics, it will never be able to reconstruct all objects perfectly. There will also be additional missing energy due to neutrinos that were produced in interactions that are not detected by any system. The amount of this lost energy in the detector can be determined from the deviation of the sum of the $p_{T}$ of the collision from zero. The $\notin_{\mathrm{T}}$ is determined from the total energy reconstructed in the calorimeter, after it is corrected to account for mis-reconstruction and for muons which will not be detected in the calorimeter. Classifying the number of objects in an events as $i$, the $\not_{\mathrm{T}}$ is defined as [72], 


$$
\mathbf{E}=-\sum_{i} \mathbf{p}_{\mathrm{i}}^{\mathrm{cal}}, \quad E_{\mathrm{T}}=\sqrt{{E_{\mathrm{x}}}^{2}+{H_{\mathrm{y}}^{2}}^{2}}
$$




\section{Chapter 5}

\section{Event simulation}

To compare the measured data to the theoretical predictions is a multiple stage process, first Monte Carlo (MC) computer simulations of the interactions of protons and anti-protons are used. These modeled events are then passed through a program that simulates how the produced particles interact in the $\mathrm{D} \varnothing$ detector. This means the MC events can be reconstructed in the same way as the data events, as described in Section 4.2. Finally, an "overlay" of measured events to this MC simulation is added, which contains all the effects we cannot easily simulate, such as noise in the detector and multiple $p \bar{p}$ interactions.

After these three stages are complete, histograms can be compared of the kinematic properties of the particles reconstructed from the measured data to the particles reconstructed from simulated SM processes. The reconstructed SM processes are classified into "signal", the process that is being searched for and "backgrounds", all other predicted processes that mimic a signal event and hence will be selected by the analysis. An example can be seen in Figure 5.1, where the measured data are compared to the predicted contributions from different SM processes.

In data each kinematic property has a certain "resolution", the width of which will be different for each simulated background, due to the different physics and 
reconstruction processes associated. There are two parts to the resolution, the detector resolution, which is due to limitations in how well the reconstruct particles and their properties can be measured, and the "physical" resolution, dependent on the process measured. This "physical" resolution can be understood in the terms of a 2-body decay, for example $W^{-} \rightarrow e^{-} \overline{\nu_{e}}$. When the mass of the $W$ boson is reconstructed it will be seen to take a range of values due to the energy lost to the neutrino.

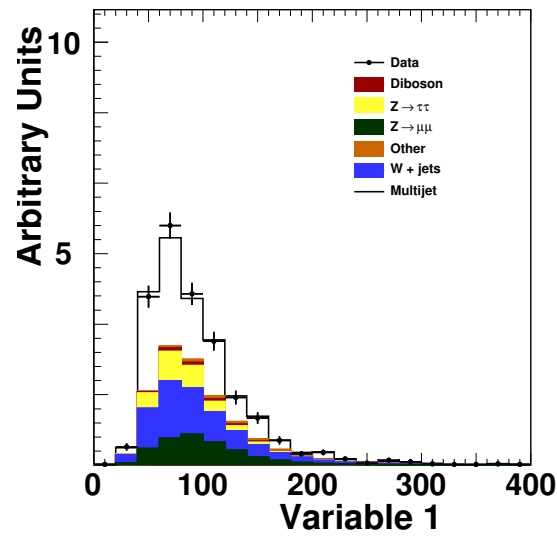

Figure 5.1: An example histogram, with the data shown as black points and the statistical error on the data as the crosses, the solid histogram shows the predicted contributions from different SM processes. Each contribution is plotted as a different colour stacked one on top of another, to show the total and individual contributions.

When comparing the histograms of the properties of data to the predicted backgrounds, mismodelings between the reconstructed data and MC simulations are often seen. For example the energy resolution is better in MC simulations than in data. Additional corrections are applied to account for these effects and are explained in detail in Section 7.4. These corrections to the MC are applied as "weights", with each correction corresponding to a specific weight. The combined effect of these is referred to as the global or event weight. 


\subsection{Monte Carlo generators}

For each proton anti-proton collision at the Tevatron the final outcome will differ. The momentum distributions of the partons will differ, as will the particle produced through the interactions and their decay products. To model what is seen in the data, one needs to simulate large (of order of 100,000 or more) numbers of MC events in order to reproduced all possible outcomes. The resulting MC samples have far larger instantaneous luminosity than the data sample, therefore must be correctly normalized to the data as described in Section 5.2.4.

The class of programs that model the outcome of the collisions are MC generators and work on the idea of random sampling. For each simulated collision there is a set range of possible inputs, which are picked at random, using numbers as determined by pseudorandom number generators. These inputs are then run through the full simulation of the modeled interactions, resulting in an output which reflects the random choice of inputs and hence models the range of outcomes observed in data.

Due to the complex nature of such an event it is generally separated into different steps, each of which is computed separately and deals with different parts of the collision and subsequent interactions and decays. The main steps are, the hard process (the proton anti-proton collision), hadronization of the produced partons, and the underlying event which contains all other interactions. Any particle with either colour or electric charge in the initial or final state can radiate photons and gluons. This initial and final state radiation must also be modeled either as part of the hard scatter or underlying event. The different parts of the interaction are shown in Figure 5.2 for the hard scatter and the underlying event and multiple interactions are shown in Figure 5.3.

- The hard scatter modeling

This is the modeling of the hard scatter and the outgoing high momentum 


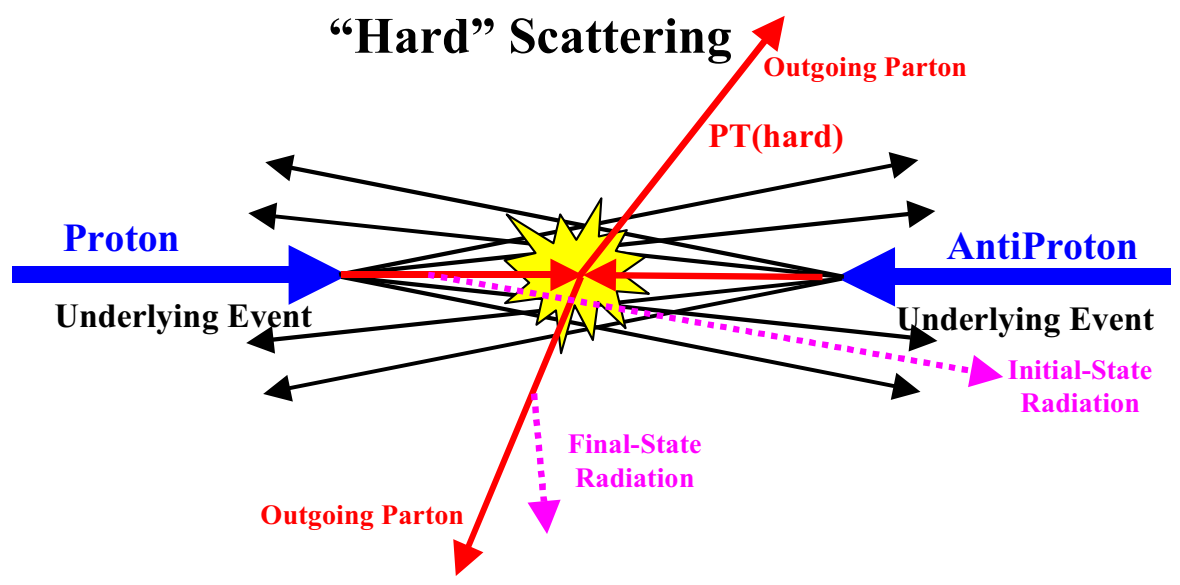

Figure 5.2: An illustration of a MC simulated hard scatter process. The three parts of the event are shown, the outgoing particles from the hard scatter (red) and the remnants of the proton and anti-proton (black). The initial and final state radiation is shown in pink [73].

particles produced. As protons are composite objects, this is in fact the interaction of quarks and gluons inside the protons. The parton distribution function, PDF, describes the momentum distribution between the quarks and gluons in the proton. The MC generator calculates the produced outgoing particles from the known matrix elements, MEs, of the interaction. The ME is known to various different levels of accuracy, with the level dependent on the MC generator used. The accuracy is determined by the order in perturbation theory of the ME calculation. The lowest accuracy or order is referred to as leading order (LO), then next-to-leading order (NLO), then next-to-next-to-leading order (NNLO), etc.

- The hadronization modeling

Once the quarks are no longer confined in the proton, then they are no longer colour neutral and they will undergo hadronization. This process, described in Section 2.3, will produce a shower of hadrons. 


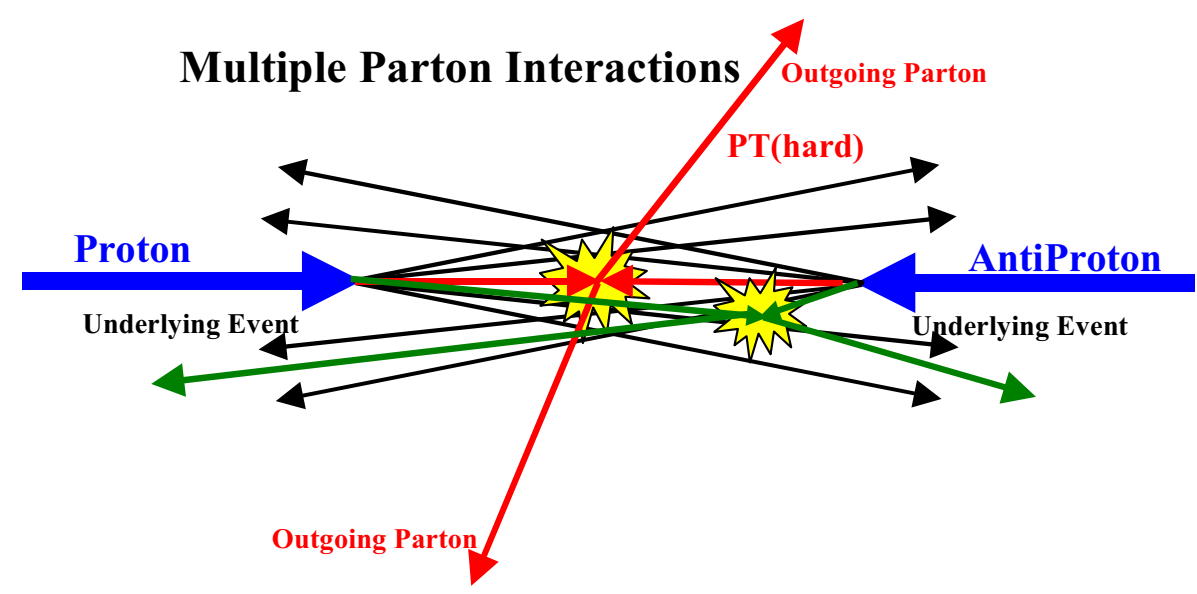

Figure 5.3: An illustration of multiple interactions within the detector, showing how in addition to the hard scatter process (red) there can be a second semi-hard scatter (green). This is classified as part of the underlying event [73].

- The underlying event modeling

The underlying event is all interactions in the event which are not in the hard scatter, i.e. the interactions due to the rest of the components of the proton and anti-proton, any additional multiple interactions due to additional hard scatters in the event and the initial and final state radiation.

Three MC generators will be described in more detail, PYTHIA [62], ALPGEN [63] and HERWIG $++[74]$.

\subsubsection{PYTHIA}

Pythia [62] is a LO MC event generator i.e the matrix elements included have been calculated only to lowest order in perturbation theory, tree level. PYTHIA contains a very comprehensive library of MEs for hard scatter processes, with over 300 separate processes stored, the majority of which are two-body $2 \rightarrow 2$ scatterings. It performs the showering and hadronization in the event associated with these MEs. The initial and final state radiation will result in events with 
higher multiplicities of particles in their final state. As PYTHIA calculates its ME to first order and this radiation depends on higher orders, PYTHIA uses the "parton shower" [75] method to model this. It is used to both calculate the QCD and the QED showering. The parton shower method uses only approximations to the full MEs, which reduces the complexity of the calculations, allowing for the modeling of the production of many partons, to produce these higher multiplicity final states [62]. PYTHIA also models the interactions of the remnants of the protons and anti-protons after the hard scatter, modeling one shower object for each remnant. In addition, PYTHIA also models multiple interactions within the collision.

PYTHIA performs its hadronization of particles by a method know as "string fragmentation" [62]. As mentioned in Section 2.3 when the partons are separated, one can imagine them being connected by a coloured object, a string of quarks and gluons. This object can be modeled as a massless relativistic string with no transverse degrees of freedom [62].

PYTHIA contains the full range of particles and decays as predicted by the SM (and if needed various extensions to it), along with the relevant branching ratios, masses, and coupling strengths for each, allowing it to correctly perform the full decay chain for the hard scattered particles.

\subsubsection{ALPGEN}

ALPGEN [63] is a LO MC event generator, using the known ME of hard scatter processes it calculates the outgoing particles and the associated initial and final radiation but does not simulate the hadronization, parton shower or the underlying event. ALPGEN generates parton level events with the full details of the colour and flavour of the particles produced, this enables matching with other event generators [63]. This allows ALPGEN to be interfaceable with other event 
generators such as PYTHIA which can take the MC events calculated by ALPGEN and simulate the hadronization, parton shower and the rest of the underlying event.

AlPGEN is designed to calculate high jet-multiplicity events with $W, Z$ or Higgs bosons. Specially of interest for the analyses performed in this thesis is the case of a $W$ or $Z$ boson production in association with a heavy bottom or charm quark pair and up to an additional four jets.

\subsubsection{HERWIG ++}

The HERWIG ++ (Hadron Emission Reactions With Interfering Gluons) [74] event generator is a multipurpose MC simulation program. Like PYTHIA it calculates both the outgoing particles associated with a specific hard scattering process and performs the showering and hadronization. The HERWIG ++ generator places specific emphasis on the detailed simulation of QCD parton showers [74], using the parton shower method as described in Section 5.1.1. The QED radiation is treated separately, by a method based on the YFS formalism [74, 76].

The simulation of the underlying event is performed using the eikonal multiple parton-parton scattering model [74]. HERWIG ++ does not store as large a library of hard scattering MEs as PYTHIA. It is designed to interface via the Les Houches Accord [77], a standardized interface between MC generators and ME generators like ALPGEn, to access specific MEs. Similar to PythiA, HERWIG ++ contains the full range of particles and decays as predicted by the SM (and extensions to it), as well as the relevant branching ratios, masses, and coupling strengths for each.

For the implementation of new BSM models, HERwIG ++ is designed so a new model can inherit from the structure already in place allowing new processes to be added easily. This structure was investigated in a project described in detail 
in Chapter 6, in which the tree level interactions associated with the NMSSM, were added into HERWIG ++ .

\subsubsection{TAUOLA}

TAUOLA [78] is used in addition to the MC event generators for the case of the tau lepton decays. TAUOLA provides correct treatment of the tau lepton polarization and also provides the branching ratios for the tau lepton decays as defined by the Particle Data Group, PDG [23]. As the tau lepton is a very short lived lepton which decays in the detector, it is important that the tau lepton polarization is treated correctly, as it can effect the properties of its decay products.

\subsection{Detector simulation}

After events have been produced by the MC generators, they need to go through an additional step before comparison with data. This step models the interactions of the particles for each MC event within the DØ detector and their reconstruction.

\subsubsection{DØgstar}

In order to model how the produced particles interact in the detector and how they would be reconstructed, the program D $\varnothing$ gstar, (D $\varnothing$ GEANT Simulation of the Total Apparatus Response), was developed [79]. This builds on the GEANT [80] package which is a detector description and simulation tool.

GEANT models the interactions within the detection systems, accurately modeling the electromagnetic and hadronic showers and the trajectory particles will take when they encounter magnetic fields. In addition it models the interactions 
with the component materials of the detector, the metal structure itself, and electronics contained within it. For the GEANT simulation to be successful, accurate information is needed on all the systems of the detector, their locations, and the complete support infrastructure for them, both in terms of the physical supports and in terms of electronics. DØ uses the version GEANT3 in its simulation.

The program $D \emptyset$ gstar uses all this information about the detector and creates a model that enables the treatment of the MC events and data to be identical, by modeling the detector signals that the MC events would have generated had they been produced in DØ. D Øgstar is separated into various parts, D Øgen simulates the geometric information of the detector and DØsim the tracking and hit simulation. DØraw adds the zero bias overlay (Section 5.2.2) as well as performing pile-up and hit digitization [79]. The final reconstruction of the $\mathrm{MC}$ events is performed with DØrec.

\subsubsection{Zero bias overlay}

There is an additional set of information that is still not included in the MC events, which is the information on detector effects and beam conditions. Unlike in the simulated MC events, the protons and anti-protons that collide in the detector are part of a beam giving rise to the possibility of additional interactions that are not properly accounted for in the MC simulations. There is, in addition, no information in the MC simulations on noise in the detector due to cosmic radiation, background radiation, or the electronics.

To account for these effects the events that pass a zero bias trigger (a trigger that ensures that events are only measured when there is a bunch crossing) are measured and used to make an overlay for the $\mathrm{MC}$ simulation. The events are processed through D $\emptyset$ rawtosim, which processes the raw data so it can be combined with the MC by D $\varnothing$ raw. Since the number of events per beam crossing 
increases with the instantaneous luminosity this overlay is stored as a function of the instantaneous luminosity. In applying this overlay to the MC events, they will retain this distribution with instantaneous luminosity, know as a luminosity profile. To achieve an accurate description of data this luminosity profile must be reweighted to match that of the measured data.

The author of this thesis was responsible for creating samples of the triggered zero bias events for the Run IIb3 and Run IIb4 data epochs and processing it with the $\mathrm{D} \emptyset$ rawtosim program enabling it to be combined with the MC by $\mathrm{D} \emptyset$ raw. The data epochs are described in Section 7.1.

\subsubsection{Trigger correction}

The applied triggers are not 100\% efficient (Section 4.1) as not all particles will be correctly reconstructed and hence a correction must be applied to the MC events to account for this. As there is no model of the $\mathrm{D} \varnothing$ trigger system the efficiencies are measured in data and then applied as weights to the MC events. There is a weight measured for each reconstructed particle in an event which is combined to give a total weight that is applied to the events. The triggers used at D $\varnothing$ have evolved over time as improvements were applied. Therefore a set of weights are stored for each trigger for different ranges of recorded data. The total weight applied is a weighted average of these weights dependent on the luminosity range used.

\subsubsection{Luminosity normalization}

A luminosity normalization must be applied to ensure the integrated luminosity of the produced MC samples matches that of the data. This luminosity weight, $W_{\text {lumi }}$, is defined as, 


$$
W_{\text {lumi }}=\frac{\sigma_{M C} L}{N_{M C}}
$$

where $\sigma_{M C}$ is the cross section of the produced sample, $L$ is the integrated luminosity of the data used, and $N_{M C}$ is the number of MC events produced.

\subsection{ROOT}

To be able to compare the measured data with the simulated events from MC a toolkit called ROOT [81] is used. This package is designed to make it simple to handle and analyze large data sets [81]. The package stores events in files know as Trees, allowing for information of objects in these events to be stored in a multiple layered structure, where the highest level is known as branches and the lower as leaves. The branches are to store separate reconstructed objects and the leaves the reconstructed properties of these objects. All histograms contained in this thesis have been drawn with this toolkit, an example is shown in Figure 5.1. 


\section{Chapter 6}

\section{NMSSM in HERWIG++}

In adding new BSM models into HERwIG++, HERWIG++ takes advantage of the inheritance mechanism of $\mathrm{C}++$, allowing one to build on the structure and models already implemented. This chapter will describe the specific case of adding the NMSSM and then describe its validation both by comparison to NMHDECAY, for two cases a set of nominal parameters and at 5 benckmark points, and within Herwig ++ at the limit where the NMSSM collapses to the MSSM [82, 83].

\subsection{Implementation of the NMSSM}

The HeRwIG ++ code structure allows BSM models to be implemented by specifying the Feynman rules as a set of Vertex classes and implementing a Model class specifying any new couplings and parameters in the model [74] [84]. HerwiG++ is designed to calculate all the two- and three-body decays and $2 \rightarrow 2$ scattering processes associated with the specified Feynman rules [84].

The use of the inheritance mechanism of $\mathrm{C}++$ means that new models being implemented do not have to start from scratch but can build on features already in place. The NMSSM Model class inherits from the MSSM Model class and from the StandardModel Model class. From the MSSM Model class it inherits the 
ability to read in supersymmetric spectra via the SUSY Les Houches Accord, SLHA, format [85] and the Vertex classes that are unchanged from the MSSM. From the StandardModel Model class it inherits the SM couplings and all Vertex classes which have not changed between the SM and the MSSM.

The NMSSM expands two sectors of the MSSM, the Higgs boson sector and the neutralino sector. The neutralino sector is expanded from four to five neutralinos. The additional neutralino and the associated mixing matrix can be incorporated in the MSSM Vertex classes, the class that governs the interactions of the neutralinos with the fermions, sfermions, and electroweak gauge bosons in the MSSM [84]. Therefore, only the Higgs boson interactions need to be implemented as new Vertex classes.

The free independent parameters of the model (not including $M_{Z}$ which is defined in the StandardModel class), which are relevant for the Higgs sector, are chosen according to the SUSY Les Houches Accord conventions [85] to be the following six parameters,

$$
\tan \beta, \lambda, \kappa, A_{\lambda}, A_{\kappa}, \mu_{e f f}
$$

This assumes that the Higgs boson mixing parameter, $\mu$, is set to zero and the unspecified parameters $m_{h_{1}}^{2}, m_{h_{2}}^{2}$ and $m_{S}^{2}$, the squared masses of the Higgs bosons, are determined from minimizing the potential. The parameter $\mu_{\text {eff }}$ is the effective $\mu$ parameter given by $\lambda\langle S\rangle$, where $\langle S\rangle$ is the VEV of the singlet Higgs field, $\tan \beta=v_{u} / v_{d}$ is the ratio of the Higgs VEVs, and $A_{\lambda}$ and $A_{\kappa}$ are the soft trilinear couplings associated with $\lambda$ and $\kappa$, respectively.

The new Higgs boson interactions in the NMSSM are implemented in a series of model specific vertex classes, i.e. NMSSMABCVertex classes, where A,B and C represent the interacting particles, as defined by the Feynman rules. Six specific tree-level interactions are required. The Feynman rules corresponding to these tree-level interactions are given in Appendix B. 
Higgs bosons to fermions: NMSSMFFHVertex

The coupling of the Higgs bosons to a SM fermion anti-fermion pair, using the tree-level vertices and including the running mass of the fermion.

Higgs bosons to gauge bosons: NMSSMVVHVertex, NMSSMVHHVertex

The interactions of the Higgs bosons with electroweak gauge bosons are included at tree level HWW, HZZ, HHW and HHZ, with no radiative or electroweak corrections included.

\section{Higgs bosons to sfermions: NMSSMHSFSFVertex}

The coupling of the Higgs bosons to scalar quarks and scalar leptons are included at tree level, with no radiative or electroweak corrections. Mixing is only included for third generation sfermions (stop, sbottom and stau).

\section{Higgs bosons to gauginos: NMSSMGOGOHVertex}

The interaction of the Higgs bosons with charginos and neutralinos are included at tree level, with no radiative or electroweak corrections.

\section{Triple Higgs bosons: NMSSMHHHVertex}

The trilinear self interactions of the Higgs bosons in the NMSSM are included as given in [7]. Radiative corrections from top and bottom loops are included as taken from NMHDECAY [86]. For the heavier Higgs bosons these can more than double the partial widths for the decay of heavier Higgs bosons into lighter ones.

In addition to these tree-level vertices, we include the loop induced coupling of the Higgs bosons to a pair of gluons or photons, which are phenomenologically important for the production and decay of the Higgs bosons.

\section{Higgs bosons to photons: NMSSMPPHVertex}

The interaction of the Higgs bosons with a pair of photons. Loop effects 
from top and bottom quarks, tau leptons, the $W$ boson, the charged Higgs bosons, charginos and stop, sbottom and stau sfermions are included for the scalar Higgs bosons. For the pseudoscalar Higgs bosons contributions from the top and bottom quarks, tau leptons and charginos are included.

\section{Higgs bosons to gluons: NMSSMGGHVertex}

The interaction of the Higgs bosons with a pair of gluons. Loop effects due to top and bottom quarks, and stop and sbottom squarks are included for scalar Higgs bosons. For the pseudoscalar Higgs bosons contributions from the top and bottom quarks are included.

\subsection{Comparison to NMHDecay}

To determine the accuracy of the programmed vertices, the partial widths for Higgs boson decays in the NMSSM as calculated by HERWIG ++ are compared to those calculated by NMHDECAY. In order for the comparison to be as reliable as possible, the input parameters and masses are set to be the same in both programs. The quark masses are set to their pole masses, as the calculation of the running masses is treated differently between the two programs. For the vertices where NMHDECAY has a higher order of radiative corrections, i.e. for Higgs bosons decays into fermions through the $h \rightarrow g g \rightarrow b \bar{b} / c \bar{c}$ interaction, these extra radiative corrections are switched off. The loop-induced coupling of the Higgs bosons to a pair of gluons or photons, is altered in NMHDECAY to match the loops included in Herwig ++ . The effect on the calculated partial widths of these additional corrections not included in HERWIG ++ is investigated. 


\section{The decay of the Higgs bosons to gluons:}

- For the decay of the scalar Higgs bosons to gluons, NMHDECAY includes effects from the charm quark and from all squarks, not the just stop and sbottom. This has a significant effect for the lightest Higgs boson introducing discrepancies at the $2 \%$ level and is negligible for the heavier Higgs bosons at $0.06 \%$.

- For the pseudoscalar Higgs bosons NMHDECAY includes the additional effect of charm quarks which introduces similar discrepancies to those for the lighter scalar Higgs bosons at around $1.5 \%$ and is negligible at high Higgs bosons masses again at the $0.06 \%$ level.

- NMHDECAY also includes effects of QCD radiative corrections, which can be shown to have a much more important effect resulting in a near doubling of the partial width of Higgs boson to gluon decays for all Higgs bosons.

\section{The decay of the Higgs bosons to photons:}

- For the decay of the scalar Higgs bosons to photons NMHDECAY includes additional effects from the charm quark and from all squarks and sleptons. The contribution of these additional particles has a minimal effect on the partial width for the decay of the light Higgs bosons around the $0.5 \%$ level, but for heavier Higgs bosons this effect increases to around $4 \%$.

- NMHDECAY includes the additional effect of charm loops in pseudoscalar Higgs boson decays, this effect is most apparent for light Higgs bosons, introducing discrepancies at the $2 \%$ level whereas for heavy pseudoscalar Higgs bosons the effect is negligible at the $0.05 \%$ level.

The input parameters used are shown in Table 6.1 , where $M_{1}, M_{2}$, and $M_{3}$ are the gaugino masses for the Bino, Wino and gluino, $A_{t}, A_{b}$, and $A_{\tau}$ are the trilinear 


\begin{tabular}{|c|c|c|c|c|c|}
\hline $\tan \beta$ & $M_{1} / \mathrm{GeV}$ & $M_{2} / \mathrm{GeV}$ & $M_{3} / \mathrm{GeV}$ & $A_{t} / \mathrm{GeV}$ & $A_{b} / \mathrm{GeV}$ \\
\hline 2 & 66 & 133 & 500 & -2500 & -2500 \\
\hline$A_{\tau} / \mathrm{GeV}$ & $A_{\lambda} / \mathrm{GeV}$ & $A_{\kappa} / \mathrm{GeV}$ & $\lambda$ & $\kappa$ & $\mu_{\mathrm{eff}} / \mathrm{GeV}$ \\
\hline-2500 & 1280 & 0.0 & 0.7 & 0.05 & 530 \\
\hline$m_{\tilde{\tau}_{L}} / \mathrm{GeV}$ & $m_{\tilde{\tau}_{R}} / \mathrm{GeV}$ & $m_{\tilde{q}_{L}} / \mathrm{GeV}$ & $m_{\tilde{u}_{R}} / \mathrm{GeV}$ & $m_{\tilde{d}_{R}} / \mathrm{GeV}$ & \\
\hline 200.0 & 200.0 & 1000.0 & 1000.0 & 1000.0 & \\
\hline
\end{tabular}

Table 6.1: NMHDECAY input parameters used, for comparison of NMHDECAY to Herwig ++. The masses are given in $\mathrm{GeV}$.

couplings of the top, bottom quarks and tau lepton all of which are defined at the input scale. The sfermion masses, the left and right scalar tau lepton masses, $m_{\tilde{\tau}_{L}}$ and $m_{\tilde{\tau}_{R}}$, the left third generation scalar quark mass, $m_{\tilde{q}_{L}}$ and the right scalar up and scalar down quark masses $m_{\tilde{u}_{R}}$ and $m_{\tilde{d}_{R}}$ must also be defined. The SM inputs used are $\alpha_{s}\left(M_{Z}\right)=1.172, M_{b}=4.214 \mathrm{GeV}$, and $M_{t}$ (pole) $=171.4 \mathrm{GeV}$.

The comparison between NMHDECAY and HERwIG++ shows a very high level of agreement between the partial widths calculated by the two programs. ${ }^{1}$ This comparison is shown for the heaviest Higgs boson in Table 6.2. The heaviest Higgs boson is presented in order to show the results across the largest number of possible decay channels. The partial widths calculated for the other Higgs bosons, scalar, pseudoscalar and charged, show the same level of agreement as is seen for the heaviest Higgs boson.

\subsection{Comparison of Herwig ++ at benchmark points}

The NMSSM in HERwIG ++ was run over five benchmark points as described in Ref. [87]. Due to the large number of free parameters in the model it is convenient to construct benchmark points of specific parameter values which embody the main phenomenological aspects of the model. Running HeRwiG ++ on these

\footnotetext{
${ }^{1}$ Some discrepancy was seen with Higgs boson decays with smuons, which was determined to be from a bug in v2.3.1 of NMHDECAY, which outputted smuon mass eigenstates instead of the helicity eigenstates. This was corrected in the version of NMHDECAY used and is fixed in later versions of NMHDECAY.
} 


\begin{tabular}{|c|c|c|c|}
\hline $\begin{array}{l}\text { Decay } \\
\text { type }\end{array}$ & $\begin{array}{l}\text { NMHDECAY } \\
\text { partial widths }\end{array}$ & $\begin{array}{l}\text { HERWIG }++ \\
\text { partial widths }\end{array}$ & $\begin{array}{l}\text { Fractional } \\
\text { difference }\end{array}$ \\
\hline$h_{3} \rightarrow g g$ & 0.01512 & 0.01512 & $1.7 \times 10^{-6}$ \\
\hline$h_{3} \rightarrow \tau^{-}, \tau^{+}$ & 0.01076 & 0.01076 & $7.8 \times 10^{-7}$ \\
\hline$h_{3} \rightarrow s, \bar{s}$ & $3.691 \times 10^{-4}$ & $3.691 \times 10^{-4}$ & $9.2 \times 10^{-7}$ \\
\hline$h_{3} \rightarrow c, \bar{c}$ & $1.257 \times 10^{-3}$ & $1.257 \times 10^{-3}$ & $1.2 \times 10^{-7}$ \\
\hline$h_{3} \rightarrow b, \bar{b}$ & 0.2556 & 0.2556 & $8.6 \times 10^{-7}$ \\
\hline$h_{3} \rightarrow W^{+}, W^{-}$ & $3.727 \times 10^{-4}$ & $3.727 \times 10^{-4}$ & $4.1 \times 10^{-6}$ \\
\hline$h_{3} \rightarrow Z^{0}, Z^{0}$ & $1.851 \times 10^{-4}$ & $1.851 \times 10^{-4}$ & $4.6 \times 10^{-6}$ \\
\hline$h_{3} \rightarrow \gamma \gamma$ & $9.958 \times 10^{-}$ & $9.958 \times 10^{-5}$ & $4.4 \times 10^{-7}$ \\
\hline$h_{3} \rightarrow h_{1}, h_{1}$ & 0.04226 & 0.04226 & $-8.9 \times 10^{-7}$ \\
\hline$h_{3} \rightarrow h_{1}, h_{2}$ & 0.3895 & 0.3895 & $-8.8 \times 10^{-7}$ \\
\hline$h_{3} \rightarrow h_{2}, h_{2}$ & 0.3895 & 0.3895 & $-8.8 \times 10^{-7}$ \\
\hline$h_{3} \rightarrow a_{1}, a_{1}$ & $1.341 \times 10^{-4}$ & $1.341 \times 10^{-4}$ & $5.5 \times 10^{-9}$ \\
\hline$h_{3} \rightarrow \tilde{\chi}_{1}^{0}, \tilde{\chi}_{1}^{0}$ & $3.448 \times 10^{-3}$ & $3.448 \times 10^{-3}$ & $1.6 \times 10^{-6}$ \\
\hline$h_{3} \rightarrow \tilde{\chi}_{1}^{0}, \tilde{\chi}_{3}^{0}$ & 0.06942 & 0.06942 & $1.8 \times 10^{-6}$ \\
\hline$h_{3} \rightarrow \tilde{\chi}_{1}^{0}, \tilde{\chi}_{4}^{0}$ & 0.01532 & 0.01532 & $2.4 \times 10^{-6}$ \\
\hline$h_{3} \rightarrow \tilde{\chi}_{2}^{0}, \tilde{\chi}_{3}^{0}$ & 0.2086 & 0.2 & $1.7 \times 10^{-6}$ \\
\hline$h_{3} \rightarrow \tilde{\chi}_{3}^{0}, \tilde{\chi}_{3}^{0}$ & 0.2607 & 0.2607 & $2.2 \times 10^{-6}$ \\
\hline$h_{3} \rightarrow \tilde{\chi}_{4}^{0}, \tilde{\chi}_{4}^{0}$ & 0.01106 & 0.01106 & $7.4 \times 10^{-6}$ \\
\hline$h_{3} \rightarrow \tilde{\chi}_{1}^{+}, \tilde{\chi}_{1}^{-}$ & 0.1844 & 0.1844 & $1.2 \times 10^{-6}$ \\
\hline$h_{3} \rightarrow \tilde{\chi}_{2}^{+}, \tilde{\chi}_{2}^{-}$ & $1.414 \times 10^{-3}$ & $1.414 \times 10^{-3}$ & $1.3 \times 10^{-5}$ \\
\hline$h_{3} \rightarrow \tilde{e}_{L}^{-}, \tilde{e}_{L}^{+}$ & $3.26 \times 10^{-3}$ & $3.26 \times 10^{-3}$ & $-4.6 \times 10^{-7}$ \\
\hline$h_{3} \rightarrow \tilde{\mu}_{L}^{-}, \tilde{\mu}_{L}^{+}$ & $3.26 \times 10^{-3}$ & $3.26 \times 10^{-3}$ & $1.1 \times 10^{-5}$ \\
\hline$h_{3} \rightarrow \tilde{e}_{R}^{-}, \tilde{e}_{R}^{+}$ & $2.087 \times 10^{-3}$ & $2.087 \times 10^{-3}$ & $-8.9 \times 10^{-7}$ \\
\hline$h_{3} \rightarrow \tilde{\mu}_{R}^{-}, \tilde{\mu}_{R}^{+}$ & $2.087 \times 10^{-3}$ & $2.087 \times 10^{-3}$ & $1.4 \times 10^{-5}$ \\
\hline$h_{3} \rightarrow \tilde{\nu}_{e L}, \tilde{\nu}_{\bar{e} L}$ & 0.01061 & 0.01061 & $-2.9 \times 10^{-7}$ \\
\hline$h_{3} \rightarrow \tilde{\nu}_{\mu L}, \tilde{\nu}_{\bar{\mu} L}$ & 0.01061 & 0.01061 & $-2.9 \times 10^{-7}$ \\
\hline$h_{3} \rightarrow \tilde{\tau}_{1}^{-}, \tilde{\tau}_{1}^{+}$ & 0.03028 & 0.03028 & $-6.7 \times 10^{-7}$ \\
\hline$h_{3} \rightarrow \tilde{\tau}_{2}^{-}, \tilde{\tau}_{2}^{+}$ & $1.414 \times 10^{-3}$ & $1.414 \times 10^{-3}$ & $1.3 \times 10^{-5}$ \\
\hline$h_{3} \rightarrow \tilde{\tau}_{1}^{-}, \tilde{\tau}_{2}^{+}$ & $5.606 \times 10^{-5}$ & $5.606 \times 10^{-5}$ & $-9.9 \times 10^{-9}$ \\
\hline$h_{3} \rightarrow \tilde{\tau}_{2}^{-}, \tilde{\tau}_{1}^{+}$ & $5.606 \times 10^{-5}$ & $5.606 \times 10^{-5}$ & $-9.9 \times 10^{-9}$ \\
\hline$h_{3} \rightarrow \tilde{\nu}_{\tau L}, \tilde{\nu}_{\bar{\tau} L}$ & 0.01061 & 0.01061 & $-2.9 \times 10^{-7}$ \\
\hline
\end{tabular}

Table 6.2: Comparison of the partial widths of the heaviest Higgs boson, $h_{3}$, as calculated by NMHDECAY and HERwiG ++ . The fractional difference between the calculated partial widths is also shown. 
points gives the opportunity to test the model over a large range of the parameter space to ensure that it functions consistently. This also ensures that all decay channels are tested, including those which do not occur in most parameter space regions. The input spectrum files are generated using NMHDECAY.

The benchmark points are constructed in the the constrained NMSSM, cNMSSM [88], as this reduces the number of input parameters required. In these scenarios the gaugino masses, trilinear couplings, scalar fermion masses and Higgs boson masses are unified at the GUT scale, $M_{G U T}$ [87],

$$
M_{1,2,3}=M_{1 / 2}, \quad M_{H_{i}}=M_{\tilde{f}}=M_{0}, \quad A_{i}=A_{0} .
$$

Five benchmark points are studied, the first three of which are in the region of parameter space where Higgs boson to Higgs boson decays are not suppressed and the last two in the region where they are suppressed.

- Point 1 - The decay $h \rightarrow a a \rightarrow 4 b$ is the dominant decay of the Higgs bososn which has properties that resemble that as expected for the SM Higgs boson, the SM-like Higgs boson.

- Point 2 - The decay $h \rightarrow a a \rightarrow 4 \tau$ is the dominant SM-like Higgs boson decay, the mass of $h_{1}$ is close to theoretical maximum (within the cNMSSM) of $130 \mathrm{GeV}$.

- Point 3 - The decay $h \rightarrow a a \rightarrow 4 \tau$ is the dominant SM-like Higgs boson decay. The mass of $h_{1}$ mass is close to theoretical minimum (within the cNMSSM) of $90 \mathrm{GeV}$.

- Point 4 - The $h_{1}$ is very light with $M_{h_{1}}<50 \mathrm{GeV}, h_{2}$ becomes the SM-like Higgs boson.

- Point 5 - In this regime all Higgs bosons are light, in the mass range 90 to $200 \mathrm{GeV}$. 


\begin{tabular}{|c|c|c|c|c|c|}
\hline & P1 & P2 & P3 & P4 & P5 \\
\hline $\operatorname{sign}\left(\mu_{e f f}\right)$ & + & + & + & - & + \\
$\tan \beta$ & 10 & 10 & 10 & 2.6 & 6 \\
$M_{0}(\mathrm{GeV})$ & 174 & 174 & 174 & 775 & 1500 \\
$M_{1 / 2}(\mathrm{GeV})$ & 500 & 500 & 500 & 760 & 175 \\
$A_{0}(\mathrm{GeV})$ & -1500 & -1500 & -1500 & -2300 & -2468 \\
$A_{\lambda}(\mathrm{GeV})$ & -1500 & -1500 & -1500 & -2300 & -800 \\
$A_{\kappa}(\mathrm{GeV})$ & -33.9 & -33.4 & -628.56 & -1170 & 60 \\
$M_{H_{d}}(\mathrm{GeV})$ & - & - & - & 880 & -311 \\
$M_{H_{d}}(\mathrm{GeV})$ & - & - & - & 2195 & 1910 \\
$\lambda$ & 0.1 & 0.1 & 0.4 & 0.53 & 0.016 \\
$m_{h_{1}}(\mathrm{GeV})$ & 120.2 & 120.2 & 89.9 & 32.3 & 90.7 \\
$m_{h_{2}}(\mathrm{GeV})$ & 998 & 998 & 964 & 123 & 118 \\
$m_{h_{3}}(\mathrm{GeV})$ & 2142 & 2142 & 1434 & 547 & 174 \\
$m_{a_{1}}(\mathrm{GeV})$ & 40.5 & 9.1 & 9.1 & 185 & 99.6 \\
$m_{a_{2}}(\mathrm{GeV})$ & 1003 & 1003 & 996 & 546 & 170 \\
$m_{H_{ \pm}}(\mathrm{GeV})$ & 1005 & 1005 & 987 & 541 & 188 \\
\hline
\end{tabular}

Table 6.3: Benchmark parameters for the five benchmark points used for validation of NMSSM, with the associated Higgs boson masses [87].

The input parameters and the Higgs boson masses associated with each point are given in Table 6.3. The partial widths per benchmark point as calculated by HERwIG ++ were compared to those as determined by NMHDECAY and are shown to have a high level of agreement for all decay modes and for all benchmark points. The comparison with HERWIG ++ partial widths for the $h_{1}$ Higgs boson can be seen in Table 6.4.

\subsection{The MSSM limit}

As a further check on the programmed model, HeRwIG++ was used to run the NMSSM in the limit when $\lambda$ and $\kappa$ go to zero and the NMSSM becomes equivalent to the MSSM. The output was compared to the values calculated using the MSSM model in HERwig++.

NMHDECAY was used to produce the spectrum files for use with both the MSSM and NMSSM. The mSUGRA model in the MSSM and the cNMSSM in 


\begin{tabular}{|c|c|c|c|c|}
\hline $\begin{array}{l}\text { Benchmark } \\
\text { Point }\end{array}$ & $\begin{array}{l}\text { Decay } \\
\text { type }\end{array}$ & $\begin{array}{l}\text { NMHDECAY } \\
\text { partial widths }\end{array}$ & $\begin{array}{l}\text { HERWIG }++ \\
\text { partial widths }\end{array}$ & $\begin{array}{c}\text { Ratio NMHDECAY } \\
\text { to HERWIG++ }\end{array}$ \\
\hline \multirow{8}{*}{ P1 } & $h_{1} \rightarrow g g$ & $1.114 \times 10^{-4}$ & $1.114 \times 10^{-4}$ & $-2.8 \times 10^{-6}$ \\
\hline & $h_{1} \rightarrow \mu^{+} \mu^{-}$ & $9.118 \times 10^{-7}$ & $9.118 \times 10^{-7}$ & $-4.3 \times 10^{-9}$ \\
\hline & $h_{1} \rightarrow \tau^{-}, \tau^{+}$ & $2.576 \times 10^{-4}$ & $2.576 \times 10^{-4}$ & $-4.5 \times 10^{-7}$ \\
\hline & $h_{1} \rightarrow s, \bar{s}$ & $8.845 \times 10^{-6}$ & $8.845 \times 10^{-6}$ & $-1.5 \times 10^{-6}$ \\
\hline & $h_{1} \rightarrow c, \bar{c}$ & $4.631 \times 10^{-4}$ & $4.631 \times 10^{-4}$ & $-2 \times 10^{-6}$ \\
\hline & $h_{1} \rightarrow b, \bar{b}$ & $6.062 \times 10^{-3}$ & $6.062 \times 10^{-3}$ & $-6.1 \times 10^{-7}$ \\
\hline & $h_{1} \rightarrow \gamma \gamma$ & $8.588 \times 10^{-6}$ & $8.588 \times 10^{-6}$ & $-2.1 \times 10^{-6}$ \\
\hline & $h_{1} \rightarrow a_{1}, a_{1}$ & 0.02646 & 0.02646 & $-1 \times 10^{-6}$ \\
\hline \multirow{9}{*}{ P2 } & $h_{1} \rightarrow g g$ & $1.114 \times 10^{-4}$ & $1.114 \times 10^{-4}$ & $-3.7 \times 10^{-6}$ \\
\hline & $h_{1} \rightarrow \mu^{+} \mu^{-}$ & $9.118 \times 10^{-7}$ & $9.118 \times 10^{-7}$ & $-9 \times 10^{-7}$ \\
\hline & $h_{1} \rightarrow \tau^{-}, \tau^{+}$ & $2.576 \times 10^{-4}$ & $2.576 \times 10^{-4}$ & $-1.2 \times 10^{-6}$ \\
\hline & $h_{1} \rightarrow s, \bar{s}$ & $8.845 \times 10^{-6}$ & $8.845 \times 10^{-6}$ & $-4.2 \times 10^{-7}$ \\
\hline & $h_{1} \rightarrow c, \bar{c}$ & $4.631 \times 10^{-4}$ & $4.631 \times 10^{-4}$ & $1.2 \times 10^{-6}$ \\
\hline & $h_{1} \rightarrow b, \bar{b}$ & $6.062 \times 10^{-3}$ & $6.062 \times 10^{-3}$ & $8 \times 10^{-7}$ \\
\hline & $h_{1} \rightarrow \gamma \gamma$ & $8.588 \times 10^{-6}$ & $8.589 \times 10^{-6}$ & $-4.2 \times 10^{-6}$ \\
\hline & $h_{1} \rightarrow a_{1}, a_{1}$ & 0.0353 & 0.0353 & $8.2 \times 10^{-7}$ \\
\hline & $h_{1} \rightarrow Z^{0}, a_{1}$ & $2.978 \times 10^{-10}$ & $2.978 \times 10^{-10}$ & $-1.4 \times 10^{-5}$ \\
\hline \multirow{8}{*}{ P3 } & $h_{1} \rightarrow g g$ & $4.396 \times 10^{-5}$ & $4.396 \times 10^{-5}$ & $-7.5 \times 10^{-7}$ \\
\hline & $h_{1} \rightarrow \tau^{-}, \tau^{+}$ & $1.745 \times 10^{-4}$ & $1.745 \times 10^{-4}$ & $1.2 \times 10^{-6}$ \\
\hline & $h_{1} \rightarrow s, \bar{s}$ & $6 \times 10^{-6}$ & $6 \times 10^{-6}$ & $5.3 \times 10^{-7}$ \\
\hline & $h_{1} \rightarrow c, \bar{c}$ & $3.414 \times 10^{-4}$ & $3.414 \times 10^{-4}$ & $-4.3 \times 10^{-7}$ \\
\hline & $h_{1} \rightarrow b, \bar{b}$ & $4.077 \times 10^{-3}$ & $4.077 \times 10^{-3}$ & $1.6 \times 10^{-6}$ \\
\hline & $h_{1} \rightarrow a_{1}, a_{1}$ & 2.288 & 2.288 & $7.6 \times 10^{-7}$ \\
\hline & $h_{1} \rightarrow \gamma \gamma$ & $2.836 \times 10^{-6}$ & $2.836 \times 10^{-6}$ & $-1.2 \times 10^{-6}$ \\
\hline & $h_{1} \rightarrow \mu^{+} \mu^{-}$ & $6.185 \times 10^{-7}$ & $6.185 \times 10^{-7}$ & $6.7 \times 10^{-7}$ \\
\hline \multirow{7}{*}{$\mathrm{P} 4$} & $h_{1} \rightarrow g g$ & $1.629 \times 10^{-7}$ & $1.629 \times 10^{-7}$ & $-8.8 \times 10^{-7}$ \\
\hline & $h_{1} \rightarrow \mu^{+} \mu^{-}$ & $2.962 \times 10^{-8}$ & $2.962 \times 10^{-8}$ & $-1.8 \times 10^{-6}$ \\
\hline & $h_{1} \rightarrow \tau^{-}, \tau^{+}$ & $8.268 \times 10^{-6}$ & $8.268 \times 10^{-6}$ & $-5.7 \times 10^{-7}$ \\
\hline & $h_{1} \rightarrow s, \bar{s}$ & $2.873 \times 10^{-7}$ & $2.873 \times 10^{-7}$ & $-8.7 \times 10^{-7}$ \\
\hline & $h_{1} \rightarrow c, \bar{c}$ & $1.418 \times 10^{-7}$ & $1.418 \times 10^{-7}$ & $-7.9 \times 10^{-7}$ \\
\hline & $h_{1} \rightarrow b, \bar{b}$ & $1.784 \times 10^{-4}$ & $1.784 \times 10^{-4}$ & $-8.1 \times 10^{-7}$ \\
\hline & $h_{1} \rightarrow \gamma \gamma$ & $1.589 \times 10^{-9}$ & $1.589 \times 10^{-9}$ & $-4.4 \times 10^{-6}$ \\
\hline \multirow{7}{*}{ P5 } & $h_{1} \rightarrow g g$ & $5.517 \times 10^{-5}$ & $5.517 \times 10^{-5}$ & $-7.2 \times 10^{-6}$ \\
\hline & $h_{1} \rightarrow \mu^{+} \mu^{-}$ & $4.141 \times 10^{-6}$ & $4.141 \times 10^{-6}$ & $-8.6 \times 10^{-7}$ \\
\hline & $h_{1} \rightarrow \tau^{-}, \tau^{+}$ & $1.17 \times 10^{-3}$ & $1.17 \times 10^{-3}$ & $-2.9 \times 10^{-6}$ \\
\hline & $h_{1} \rightarrow s, \bar{s}$ & $4.017 \times 10^{-5}$ & $4.017 \times 10^{-5}$ & $-6.1 \times 10^{-7}$ \\
\hline & $h_{1} \rightarrow c, \bar{c}$ & $3.852 \times 10^{-4}$ & $3.852 \times 10^{-4}$ & $-3.6 \times 10^{-6}$ \\
\hline & $h_{1} \rightarrow b, \bar{b}$ & 0.0275 & 0.0275 & $-2.3 \times 10^{-6}$ \\
\hline & $h_{1} \rightarrow \gamma \gamma$ & $7.825 \times 10^{-6}$ & $7.825 \times 10^{-6}$ & $-9.1 \times 10^{-6}$ \\
\hline
\end{tabular}

Table 6.4: Comparison of the lightest Higgs boson decay widths, at the five benchmark points. The fractional difference between the calculated partial widths is also shown. 
the NMSSM are used. The mSUGRA model constrains the MSSM parameters as described for the NMSSM in Section 6.3. The values for the input parameters that are used for both models can be seen in Table 6.5 (the parameters $A_{\lambda}, A_{\kappa}, \lambda$, and $\kappa$ are not used by the MSSM).

\begin{tabular}{|c|c|}
\hline $\operatorname{sign}\left(\mu_{\text {eff }}\right)$ & + \\
$\tan \beta$ & 10 \\
$M_{0} / \mathrm{GeV}$ & 174 \\
$M_{1 / 2} / \mathrm{GeV}$ & 500 \\
$A_{0} / \mathrm{GeV}$ & -1500 \\
$A_{\lambda} / \mathrm{GeV}$ & -1500 \\
$A_{\kappa} / \mathrm{GeV}$ & -628.56 \\
$\lambda$ & $\times 10^{-8}$ \\
$\kappa$ & $\times 10^{-8}$ \\
\hline
\end{tabular}

Table 6.5: NMHDECAY input parameters to calculate the MSSM and NMSSM spectrum files, for comparison in the MSSM limit.

The SM inputs used are $\alpha_{s}\left(M_{Z}\right)=1.172, M_{b}=4.214 \mathrm{GeV}$, and $M_{t}($ pole $)=$ 171.4 GeV. The NMHDECAY output, when run in the mSUGRA model, is at the GUT scale so values of the NMSSM parameters at the SUSY scale need to be determined for the input spectrum file to HERwIG ++, these are calculated using a modified NMHDECAY. The additional parameter for the MSSM, the Higgs boson mixing angle, was determined from the NMHDECAY scalar Higgs boson mixing matrix, with $\lambda$ and $\kappa$ set sufficiently small that one could be certain to be in the MSSM regime.

Doing this the Herwig ++ MSSM results were reproduced with a maximum variance of better than $10^{-5}$, showing excellent agreement, as can be see in Table 6.6. 


\begin{tabular}{|l|c|c|c|}
\hline \multicolumn{1}{|c|}{ Decay type } & Partial widths MSSM & $\begin{array}{c}\text { Partial widths NMSSM } \\
\text { in MSSM limit }\end{array}$ & $\begin{array}{c}\text { Fractional } \\
\text { difference }\end{array}$ \\
\hline$h_{2} \rightarrow \tilde{t}_{1}, \tilde{\bar{t}}_{1}$ & 2.64 & 2.64 & $1.1 \times 10^{-6}$ \\
$h_{2} \rightarrow \tilde{e}_{L}^{-}, \tilde{e}_{L}^{+}$ & $1.845 \times 10^{-4}$ & $1.845 \times 10^{-4}$ & $1.3 \times 10^{-6}$ \\
$h_{2} \rightarrow \tilde{e}_{R}, \tilde{e}_{R}^{+}$ & $1.519 \times 10^{-4}$ & $1.519 \times 10^{-4}$ & $-2.1 \times 10^{-6}$ \\
$h_{2} \rightarrow \tilde{\nu}_{e L}, \tilde{\nu}_{\bar{e} L}$ & $6.131 \times 10^{-4}$ & $6.131 \times 10^{-4}$ & $-2.1 \times 10^{-7}$ \\
$h_{2} \rightarrow \tilde{\mu}_{L}^{-}, \tilde{\mu}_{L}^{+}$ & $1.844 \times 10^{-4}$ & $1.844 \times 10^{-4}$ & $1.5 \times 10^{-6}$ \\
$h_{2} \rightarrow \tilde{\mu}_{R}^{-}, \tilde{\mu}_{R}^{+}$ & $2.742 \times 10^{-6}$ & $2.742 \times 10^{-6}$ & $-9.8 \times 10^{-7}$ \\
$h_{2} \rightarrow \tilde{\nu}_{\mu L}, \tilde{\nu}_{\bar{\mu} L}$ & $6.131 \times 10^{-4}$ & $6.131 \times 10^{-4}$ & $-2.1 \times 10^{-7}$ \\
$h_{2} \rightarrow \tilde{\tau}_{1}^{-}, \tilde{\tau}_{1}^{+}$ & 0.0535 & 0.0535 & $1.8 \times 10^{-7}$ \\
$h_{2} \rightarrow \tilde{\tau}_{2}^{-}, \tilde{\tau}_{2}^{+}$ & 0.03146 & 0.03146 & $1.1 \times 10^{-6}$ \\
$h_{2} \rightarrow \tilde{\tau}_{2}^{+}, \tilde{\tau}_{1}^{-}$ & 0.1874 & 0.1874 & $1.6 \times 10^{-6}$ \\
$h_{2} \rightarrow \tilde{\tau}_{2}^{-}, \tilde{\tau}_{1}^{+}$ & 0.1874 & 0.1874 & $1.6 \times 10^{-6}$ \\
$h_{2} \rightarrow \tilde{\nu}_{\tau L}, \tilde{\nu}_{\bar{\tau} L}$ & $6.343 \times 10^{-4}$ & $6.343 \times 10^{-4}$ & $2.9 \times 10^{-7}$ \\
$h_{2} \rightarrow \tilde{\chi}_{1}^{+}, \tilde{\chi}_{1}^{-}$ & 0.03964 & 0.03964 & $6.2 \times 10^{-7}$ \\
$h_{2} \rightarrow \tilde{\chi}_{1}^{0}, \tilde{\chi}_{1}^{0}$ & $4.161 \times 10^{-3}$ & $4.161 \times 10^{-3}$ & $8.4 \times 10^{-7}$ \\
$h_{2} \rightarrow \tilde{\chi}_{1}^{0}, \tilde{\chi}_{2}^{0}$ & 0.02132 & 0.02132 & $-6.7 \times 10^{-7}$ \\
$h_{2} \rightarrow \tilde{\chi}_{2}^{0}, \tilde{\chi}_{2}^{0}$ & 0.01921 & 0.01921 & $2.2 \times 10^{-6}$ \\
$h_{2} \rightarrow h_{1}, h_{1}$ & $3.786 \times 10^{-3}$ & $3.786 \times 10^{-3}$ & $5.1 \times 10^{-7}$ \\
$h_{2} \rightarrow b, \bar{b}^{-3}$ & 5.008 & 5.008 & $-3.2 \times 10^{-7}$ \\
$h_{2} \rightarrow t, \bar{t}$ & 0.5109 & 0.5109 & $3.9 \times 10^{-7}$ \\
$h_{2} \rightarrow \tau^{-}, \tau^{+}$ & 0.2109 & 0.2109 & $9 \times 10^{-8}$ \\
$h_{2} \rightarrow W^{+}, W^{-}$ & $1.274 \times 10^{-3}$ & $1.274 \times 10^{-3}$ & $-1.6 \times 10^{-7}$ \\
$h_{2} \rightarrow Z^{0}, Z^{0}$ & $6.302 \times 10^{-4}$ & $6.302 \times 10^{-4}$ & $1.1 \times 10^{-6}$ \\
$h_{2} \rightarrow g, g$ & $6.585 \times 10^{-3}$ & $6.585 \times 10^{-3}$ & $7.3 \times 10^{-8}$ \\
$h_{2} \rightarrow \gamma, \gamma$ & $3.859 \times 10^{-5}$ & $3.859 \times 10^{-5}$ & $1.4 \times 10^{-7}$ \\
\hline
\end{tabular}

Table 6.6: Comparison of determined partial widths for both the MSSM and the NMSSM in the MSSM limit, as calculated by HERwIG ++ and the fractional difference between them. 


\section{Chapter 7}

\section{Analysis Methods}

This section will explain the analysis method and tools used for the searches performed. Though the method covered will be specific to the analyses presented here, the general outline of it is applicable to most searches at hadron colliders.

\subsection{Data samples}

The data recorded by $\mathrm{D} \varnothing$ is separated into different subsets or data epochs reflecting the Tevatron Runs, with the analyses presented here conducted with Run IIb data. Depending on when they were recorded, the Run IIb data is classified into four epochs, for which the average delivered luminosity and the detector performance are not constant. Separating into epochs allows for separate weights and corrections to be easily applied to MC events, to reflect these changes. The different data taking epochs with their corresponding integrated luminosity are listed in Table 7.1.

\subsubsection{Data skims}

The DØ Common Samples Group, CSG, provides for each data epoch, data files in a Common Analysis Format, CAF, that place basic selections on the data. The 


\begin{tabular}{l|c|c} 
Data Epoch & $\begin{array}{c}\text { Integrated luminosity } \\
\text { (recorded, good) }\left[\mathrm{pb}^{-1}\right]\end{array}$ & Percentage used in analyses \\
\hline \hline Run I & 130 & Not used \\
Run IIa & 1078 & Not used \\
Run IIb1 & 1223 & $100 \%$ \\
Run IIb2 & 3035 & $100 \%$ \\
Run IIb3 & 1994 & $100 \%$ \\
Run IIb4 & 2404 & $100 \% / 32 \%$
\end{tabular}

Table 7.1: The integrated luminosity recorded for the different Run periods at DØ. For the search for the SM Higgs boson all of Run IIb data are used, totaling $L=8.6 \mathrm{fb}^{-1}$, whereas for the search for a $H^{ \pm \pm}$boson only the first $0.7 \mathrm{fb}^{-1}$ of Run IIb4 data is used resulting in a total integrated luminosity of $L=7.0 \mathrm{fb}^{-1}$.

samples used in these analyses are the MUinclusive (PASS 2/ 4/5/6 for Run IIb $1 / 2 / 3 / 4$ periods, respectively) samples, for which events must contain at least one muon. Data samples that have been treated in this way are referred to as skims.

This MUinclusive skim then undergoes further processing to produce subskims that contain at least one muon and at least one hadronically decaying tau lepton per event, the MuTau skim, which is skimmed separately for the Run IIb1+2, Run IIb3, and Run IIb4 data taking periods. To be selected for these these skims the muons and hadronically decaying tau leptons need to pass the following requirements:

\section{- Muon:}

A logical "OR" of the following criteria is required:

- One or more muons of identification quality "Loose", isolation quality "TopScaledMedium", and track quality "TrackLoose" (see Section 4.3.1), with a $p_{T}>12 \mathrm{GeV}$.

- One or more muons of identification quality "Loose", isolation quality "NPTight", and track quality "TrackLoose" (see Section 4.3.1), with 
a $p_{T}>12 \mathrm{GeV}$.

Only one of these quality criteria is required at pre-selection level. The logical OR at skimming level enables these skims to be applicable to all analyses.

\section{- Hadronically decaying tau lepton:}

The hadronically decaying tau lepton candidate is required to match the following standard tau lepton identification criteria [61],

- All tau leptons are required to have a $p_{T}>7 \mathrm{GeV}$.

- All tau leptons must satisfy $p_{T}^{\text {track }}>7 / 5 / 7 \mathrm{GeV}$ for the three tau lepton types Type-1/Type-2/Type-3, respectively.

- For tau leptons of Type-3, the sum of the track momenta must satisfy, $\Sigma_{\text {tracks }}\left(p_{T}\right)>10 \mathrm{GeV}$.

The $p_{T}$ is classified as the tau lepton momentum as determined in the calorimeter, whereas the momentum as determined from the highest $p_{T}$ tau lepton track is referred to as $p_{T}^{\text {track }}$.

\subsubsection{Bad data}

When noticed, either while data taking or during reconstruction, that part of the DØ detector was malfunctioning, data events are classified as "bad". There are separate "bad" flags for the calorimeter, SMT, CFT, muon systems, and triggers. Any event that was flagged as bad was rejected. Events flagged "bad" for the following common detector problems at DØ,e.g., calorimeter noise, (cal_empty_crate, cal_ring_of_fire, cal_noon_noise, spanish_fan, sca_failure and cal_coherent_noise), are rejected [89]. The luminosity normalization of the MC samples is calculated after these events have been removed. 


\subsection{SingleMuonOR trigger}

The "SingleMuonOR" trigger is a logical OR of all DØ triggers that require there to be least one muon in the event [90] as listed in Table 7.2.

Single muon triggers

\begin{tabular}{l}
\hline \hline MUHI1_ITLM10 MUHI1_TK12_TLM12 \\
MUHI1_ILM15 MUHI2_ITLM10 MUHI2_TK12_TLM12 MUHI2_ILM15 \\
MUHI3_ITLM10 MUHI3_TK12_TLM12 MUHI3_ILM15 \\
MUHI1_ILM10 MUHI2_ILM10 MUHI1_TLM12 MUHI2_TLM12 \\
MUHI1_MM10 MUHI1_TMM10 MUHI2_MM10 MUHI2_TMM10 \\
MUHI3_TMM10 MUHI3_MM10 MUHI3_ILM10 MUHI3_TLM12 \\
MUHI4_TMM10 MUHI4_MM10 MUHI4_ILM10 MUHI4_TLM12 MUHI4_ITLM10 \\
MUHI5_TMM10 MUHI5_MM10 MUHI5_ILM10 MUHI5_TLM12 MUHI5_ITLM10 \\
MUHI6_TMM10 MUHI6_MM10 MUHI6_ILM10 MUHI6_TLM12 MUHI6_ITLM10
\end{tabular}

Table 7.2: The 36 single muon triggers that comprise the SingleMuonOR trigger.

To account for the effect of requiring that the data events pass these triggers, trigger efficiencies are applied to the MC events [56], as discussed in Section 5.2.3. For the measured trigger efficiencies to be applicable to the MC samples, all three layers of the triggering system must have registered the selected muon (or muons). The efficiency is applied as a global weight, which is determined for all single muon triggers combined, for each data taking epoch.

\subsection{Monte Carlo samples}

The MC samples used in the analyses covered in this thesis are produced by two MC generators, ALPGEN and PYTHiA, as described in Sections 5.1.2 and 5.1.1, respectively. The following versions of the MC generators were used, ALPGEN version 2.11 [63] and PYTHIA version 6.323 [62]. Both MC generators use the CTEQ6L1 parton distribution functions, PDFs [91] and the tau lepton decays are 
performed by TAUOLA [78]. The SM processes that are relevant for an analysis with two hadronically decaying tau leptons and one muon in the final state, can arise in two ways. Backgrounds processes that produce two hadronically decaying tau leptons and one muon and background processes that mimic these events, either by a low multiplicity jet being misidentified as one or both of the hadronically decaying tau leptons, $\tau_{\text {fake}}$, or a muon produced in heavy quark decay that fails the muon isolation requirements, $\mu_{\text {fake }}$. The relevant background processes are:

- $\mathrm{Z}$ boson and Drell-Yan $\left(\mathrm{Z} / \gamma^{*}\right)$ production decaying to $\tau \tau, \mu \mu$, and ee lepton pairs

$Z / \gamma^{*} \rightarrow \tau \tau$ decays will be selected when both tau leptons decay hadronically and an additional $\mu_{\text {fake }}$ is selected. They can also be selected when one tau lepton decays to a muon and a $\tau_{\text {fake }}$ lepton is also present. $Z / \gamma^{*} \rightarrow \mu \mu$ decays can be selected with two additional $\tau_{\text {fake }}$ leptons in the event or with one of the muons misidentified as a hadroncially decaying tau lepton. A very small contribution from $Z / \gamma^{*} \rightarrow e e$ is also expected when the electrons are misidentified as tau leptons, with a $\mu_{\text {fake }}$ selected.

- $\mathrm{W}+$ jets production

These decays will most commonly be selected when the $W$ boson decays to a muon and two $\tau_{\text {fake }}$ leptons are selected.

\section{- Diboson (WW, WZ, and ZZ) production}

Diboson decays can produce two hadronically decaying tau leptons and a muon and therefore will be a large contribution to the selected background events.

\section{- Top-antitop quark pairs, $\mathrm{t} \overline{\mathrm{t}}$ production}

Top quarks decay to a $W$ boson and $b$ quarks and hence can produce tau 
leptons and muons in the final state. The contribution from these decays is expected to be small.

\section{- Instrumental multijet background}

Multijet events are created in the detector and are reconstructed as a $\tau_{\text {fake }}$. If a $\mu_{\text {fake }}$ is also created in an event, this event will be selected. These events are not easily modeled by MC simulations and are determined using a data driven approch.

The $Z / \gamma^{*}, W+$ jets and $t \bar{t}$ background samples are simulated using the ALPGEN MC generator with the hadronization and showering performed by PYTHIA. The diboson samples are generated entirely by PYTHIA. The samples generated are listed in Tables 7.3 and 7.4. The instrumental multijet background is estimated directly from the data and is detailed in Section 7.8.

The ALPGEN samples are generated at LO, whereas the cross sections of the samples used are available at higher orders, up to NNLO. Therefore, the cross sections of the samples are scaled up to their higher order cross sections by a k-factor, as given in Table 7.5. The higher order values are calculated separately for the different processes, with all processes using the MSTW2008 PDF set. The $Z / \gamma^{*}$ and $W+$ jets processes are normalized to the NNLO cross sections [92], as calculated by MCFM [93] (Monte Carlo for FeMtobarn processes), which is a program specifically designed to calculate such higher order cross sections. The $Z / \gamma^{*}$ and $W+$ jets heavy flavour (with two additional $b$ or $c$ quarks) events are reweighted using the ratio of NLO to LO cross sections as obtained from MCFM [93, 94]. The calculation of Langenfeld, Moch, and Uwer [95], which is combination of NNLO and Next-to-Next-to-Leading Log and a top mass of 173 $\pm 1.2 \mathrm{GeV}$, are used for $t \bar{t}$ production.

As described in Section 5.2, MC samples go through reconstruction before comparison with the data. Different releases of the $\mathrm{D} \varnothing$ reconstruction software 


\begin{tabular}{|c|c|c|c|}
\hline Process & Generator & Mass Range $[\mathrm{GeV}]$ & $\sigma \times \mathrm{BR}[\mathrm{pb}]$ \\
\hline$\overline{Z Z / \gamma^{*} \rightarrow \ell \ell+0 \operatorname{lp}}$ & ALPGEN + PYTHIA & $1015<M(\tau \tau)<75$ & 338.2 \\
\hline$Z / \gamma^{*} \rightarrow \ell \ell+1 \mathrm{lp}$ & ALPGEN + PYTHIA & $15<M(\tau \tau)<75$ & 40.0 \\
\hline$Z / \gamma^{*} \rightarrow \ell \ell+2 \mathrm{lp}$ & ALPGEN + PYTHIA & $15<M(\tau \tau)<75$ & 10 \\
\hline$Z / \gamma^{*} \rightarrow \ell \ell+3 \mathrm{lp}$ & ALPGEN + PYTHIA & $15<M(\tau \tau)<75$ & 2.76 \\
\hline$Z / \gamma^{*} \rightarrow \ell \ell+0 \mathrm{lp}$ & ALPGEN + PYTHIA & $75<M(\tau \tau)<130$ & 133.3 \\
\hline$Z / \gamma^{*} \rightarrow \ell \ell+11 p$ & ALPGEN + PYTHIA & $75<M(\tau \tau)<130$ & 40.3 \\
\hline$Z / \gamma^{*} \rightarrow \ell \ell+2 \mathrm{lp}$ & ALPGEN + PYTHIA & $75<M(\tau \tau)<130$ & 9.99 \\
\hline$Z / \gamma^{*} \rightarrow \ell \ell+3 \mathrm{lp}$ & ALPGEN + PYTHIA & $75<M(\tau \tau)<130$ & 3.09 \\
\hline$Z / \gamma^{*} \rightarrow \ell \ell+0 \mathrm{lp}$ & ALPGEN + PYTHIA & $130<M(\tau \tau)<250$ & 0.86 \\
\hline$Z / \gamma^{*} \rightarrow \ell \ell+1 \mathrm{lp}$ & ALPGEN + PYTHIA & $130<M(\tau \tau)<250$ & 0.37 \\
\hline$Z / \gamma^{*} \rightarrow \ell \ell+2 \mathrm{lp}$ & ALPGEN + PYTHIA & $130<M(\tau \tau)<250$ & 0.10 \\
\hline$Z / \gamma^{*} \rightarrow \ell \ell+3 \mathrm{lp}$ & ALPGEN + PYTHIA & $130<M(\tau \tau)<250$ & 0.03 \\
\hline$Z / \gamma^{*} \rightarrow \ell \ell+0 \mathrm{lp}$ & ALPGEN + PYTHIA & $250<M(\tau \tau)<1960$ & 0.07 \\
\hline$Z / \gamma^{*} \rightarrow \ell \ell+11 p$ & ALPGEN + PYTHIA & $250<M(\tau \tau)<1960$ & 0.03 \\
\hline$Z / \gamma^{*} \rightarrow \ell \ell+2 \mathrm{lp}$ & ALPGEN + PYTHIA & $250<M(\tau \tau)<1960$ & 0.01 \\
\hline$Z / \gamma^{*} \rightarrow \ell \ell+3 \mathrm{lp}$ & ALPGEN + PYTHIA & $250<M(\tau \tau)<1960$ & $3.9 \times 10^{-3}$ \\
\hline$Z / \gamma^{*} \rightarrow \ell \ell+2 b+01 \mathrm{p}$ & ALPGEN + PYTHIA & $15<M(\tau \tau)<75$ & 0.51 \\
\hline$Z / \gamma^{*} \rightarrow \ell \ell+2 b+1 \mathrm{lp}$ & ALPGEN + PYTHIA & $15<M(\tau \tau)<75$ & 0.20 \\
\hline$Z / \gamma^{*} \rightarrow \ell \ell+2 b+2 \mathrm{lp}$ & ALPGEN + PYTHIA & $15<M(\tau \tau)<75$ & 0.08 \\
\hline$Z / \gamma^{*} \rightarrow \ell \ell+2 b+0 \mathrm{lp}$ & ALPGEN + PYTHIA & $75<M(\tau \tau)<130$ & 0.42 \\
\hline$Z / \gamma^{*} \rightarrow \ell \ell+2 b+11 p$ & ALPGEN + PYTHIA & $75<M(\tau \tau)<130$ & 0.20 \\
\hline$Z / \gamma^{*} \rightarrow \ell \ell+2 b+2 \mathrm{lp}$ & ALPGEN + PYTHIA & $75<M(\tau \tau)<130$ & 0.10 \\
\hline$Z / \gamma^{*} \rightarrow \ell \ell+2 b+0 \mathrm{lp}$ & ALPGEN + PYTHIA & $130<M(\tau \tau)<250$ & $3.4 \times 10^{-3}$ \\
\hline$Z / \gamma^{*} \rightarrow \ell \ell+2 b+11 p$ & ALPGEN + PYTHIA & $130<M(\tau \tau)<250$ & $1.8 \times 10^{-3}$ \\
\hline$Z / \gamma^{*} \rightarrow \ell \ell+2 b+2 \mathrm{lp}$ & ALPGEN + PYTHIA & $130<M(\tau \tau)<250$ & $8.8 \times 10^{-4}$ \\
\hline$Z / \gamma^{*} \rightarrow \ell \ell+2 b+0 \mathrm{lp}$ & ALPGEN + PYTHIA & $250<M(\tau \tau)<1960$ & $3.4 \times 10^{-4}$ \\
\hline$Z / \gamma^{*} \rightarrow \ell \ell+2 b+11 p$ & ALPGEN + PYTHIA & $250<M(\tau \tau)<1960$ & $1.7 \times 10^{-4}$ \\
\hline$Z / \gamma^{*} \rightarrow \ell \ell+2 b+2 \mathrm{lp}$ & ALPGEN + PYTHIA & $250<M(\tau \tau)<1960$ & $1.1 \times 10^{-4}$ \\
\hline$Z / \gamma^{*} \rightarrow \ell \ell+2 c+0 l p$ & ALPGEN + PYTHIA & $15<M(\tau \tau)<75$ & 4.14 \\
\hline$Z / \gamma^{*} \rightarrow \ell \ell+2 c+1 \mathrm{lp}$ & ALPGEN + PYTHIA & $15<M(\tau \tau)<75$ & 0.95 \\
\hline$Z / \gamma^{*} \rightarrow \ell \ell+2 c+2 \mathrm{lp}$ & ALPGEN + PYTHIA & $15<M(\tau \tau)<75$ & 0.34 \\
\hline$Z / \gamma^{*} \rightarrow \ell \ell+2 c+0 \mathrm{lp}$ & ALPGEN + PYTHIA & $75<M(\tau \tau)<130$ & 0.93 \\
\hline$Z / \gamma^{*} \rightarrow \ell \ell+2 c+11 p$ & ALPGEN + PYTHIA & $75<M(\tau \tau)<130$ & 0.55 \\
\hline$Z / \gamma^{*} \rightarrow \ell \ell+2 c+2 \mathrm{lp}$ & ALPGEN + PYTHIA & $75<M(\tau \tau)<130$ & 0.28 \\
\hline$Z / \gamma^{*} \rightarrow \ell \ell+2 c+0 \mathrm{lp}$ & ALPGEN + PYTHIA & $130<M(\tau \tau)<250$ & $7.6 \times 10^{-3}$ \\
\hline$Z / \gamma^{*} \rightarrow \ell \ell+2 c+11 p$ & ALPGEN + PYTHIA & $130<M(\tau \tau)<250$ & $4.4 \times 10^{-3}$ \\
\hline$Z / \gamma^{*} \rightarrow \ell \ell+2 c+2 \mathrm{lp}$ & ALPGEN + PYTHIA & $130<M(\tau \tau)<250$ & $2.8 \times 10^{-3}$ \\
\hline$Z / \gamma^{*} \rightarrow \ell \ell+2 c+0 \mathrm{lp}$ & ALPGEN + PYTHIA & $250<M(\tau \tau)<1960$ & $6.2 \times 10^{-4}$ \\
\hline$Z / \gamma^{*} \rightarrow \ell \ell+2 c+1 l p$ & ALPGEN + PYTHIA & $250<M(\tau \tau)<1960$ & $4.4 \times 10^{-4}$ \\
\hline$Z / \gamma^{*} \rightarrow \ell \ell+2 c+2 \mathrm{lp}$ & ALPGEN + PYTHIA & $250<M(\tau \tau)<1960$ & $2.6 \times 10^{-4}$ \\
\hline
\end{tabular}

Table 7.3: The generated MC samples for $Z / \gamma^{*}$ processes, with the generators used and the product of the cross section and branching ratio in pb, where $\ell \ell$ can be either $e e, \mu \mu$, or $\tau \tau$ lepton pairs. The samples are generated with additional light partons (lp) and with two additional $b$ or $c$ quarks. 


\begin{tabular}{|l|c|c|}
\hline Process & Generator & $\sigma \times \mathrm{xR}[\mathrm{pb}]$ \\
\hline \hline$W \rightarrow \ell \nu+0 \mathrm{lp}$ & ALPGEN + PYTHIA & 5713 \\
$W \rightarrow \ell \nu+1 \mathrm{lp}$ & ALPGEN + PYTHIA & 1618 \\
$W \rightarrow \ell \nu+2 \mathrm{lp}$ & ALPGEN + PYTHIA & 384.5 \\
$W \rightarrow \ell \nu+3 \mathrm{lp}$ & ALPGEN + PYTHIA & 92 \\
$W \rightarrow \ell \nu+4 \mathrm{lp}$ & ALPGEN + PYTHIA & 21 \\
$W \rightarrow \ell \nu+5 \mathrm{lp}$ & ALPGEN + PYTHIA & 6.5 \\
$W+2 b \rightarrow \ell \nu+2 b+0 \mathrm{lp}$ & ALPGEN + PYTHIA & 13.95 \\
$W+2 b \rightarrow \ell \nu+2 b+1 \mathrm{lp}$ & ALPGEN + PYTHIA & 6.12 \\
$W+2 b \rightarrow \ell \nu+2 b+2 \mathrm{lp}$ & ALPGEN + PYTHIA & 2.37 \\
$W+2 b \rightarrow \ell \nu+2 b+3 \mathrm{lp}$ & ALPGEN + PYTHIA & 1.10 \\
$W+2 c \rightarrow \ell \nu+2 c+0 \mathrm{lp}$ & ALPGEN + PYTHIA & 34.35 \\
$W+2 c \rightarrow \ell \nu+2 c+1 \mathrm{lp}$ & ALPGEN + PYTHIA & 19.83 \\
$W+2 c \rightarrow \ell \nu+2 c+2 \mathrm{lp}$ & ALPGEN + PYTHIA & 8.13 \\
$W+2 c \rightarrow \ell \nu+2 c+3 \mathrm{lp}$ & ALPGEN + PYTHIA & 3.54 \\
\hline$t \bar{t}+0 \mathrm{lp} \rightarrow 2 b+2 l 2 \nu$ & ALPGEN + PYTHIA & 0.35 \\
$t \bar{t}+0 \mathrm{lp} \rightarrow 2 b+4 \mathrm{lp}$ & ALPGEN + PYTHIA & 2.27 \\
$t \bar{t}+0 \mathrm{lp} \rightarrow 2 b+l \nu+2 \mathrm{lp}$ & ALPGEN + PYTHIA & 2.24 \\
\hline$W W \rightarrow$ incl. & PYTHIA & 11.6 \\
$W Z \rightarrow$ incl. & PYTHIA & 3.25 \\
$Z Z \rightarrow$ incl. & PYTHIA & 1.33 \\
\hline
\end{tabular}

Table 7.4: The generated MC samples, for $W+$ jets, top quark and diboson processes with the generators used and the product of the cross section and branching ratio in $\mathrm{pb}$, where $\ell$ can be either $e, \mu$, or $\tau$ leptons. The $W+$ jets samples are generated with with a number of light partons (lp) and additionally with two additional $b$ or $c$ quarks. For the diboson ( $W W, W Z$ or $Z Z$ ) samples all the decays to leptons are allowed (incl).

have been developed to model the different data epochs, reflecting differences with the detector due to aging and due to increases in the average instantaneous luminosity.

\subsection{Monte Carlo correction factors}

As mentioned in Section 5.2, in order for the MC events to accurately describe the data, additional corrections are needed on top of the standard reconstruction, trigger efficiency, and luminosity weights. The relevant corrections are described 


\begin{tabular}{|l|c|}
\hline Process & k-factor \\
\hline \hline$Z / \gamma^{*} \rightarrow \ell \ell+\mathrm{Nlp}$ & 1.3 \\
$Z / \gamma^{*} \rightarrow \ell \ell+2 b+\mathrm{Rlp}$ & $1.3 \times 1.52$ \\
$Z / \gamma^{*} \rightarrow \ell \ell+2 c+\mathrm{Rlp}$ & $1.3 \times 1.67$ \\
\hline$W+j e t \rightarrow \ell \nu+\mathrm{Mlp}$ & 1.3 \\
$W+j e t \rightarrow \ell \nu+2 b+\mathrm{Nlp}$ & $1.3 \times 1.47$ \\
$W+j e t \rightarrow \ell \nu+2 c+\mathrm{Nlp}$ & $1.3 \times 1.47$ \\
\hline$t \bar{t}$ & 1.43 \\
\hline
\end{tabular}

Table 7.5: The calculated k-factors used to correct the ALPGEN LO cross section to the high order cross section, for the $Z / \gamma^{*}, W+$ jet, and $t \bar{t}$ samples. The reweighting is not dependent on the number of light partons, where $\mathrm{N}=0-3, \mathrm{M}$ $=0-5$ and $\mathrm{R}=0-2$. The $t \bar{t}$ reweightings are the same for all decay channels.

in this section.

\subsubsection{Beam position}

The interaction region at $\mathrm{D} \varnothing$ is modeled as a Gaussian function. It has been observed in data that the width of the distribution of the primary vertices varies depending on the instantaneous luminosity [96], hence this modeling will not correctly represent what is observed in data. A correction is derived from the zero bias events recorded at $\mathrm{D} \varnothing$, by fitting to the observed distribution of the primary vertices in $z$ [96]. This correction is applied as a function of the data taking epoch and the instantaneous luminosity.

\subsection{2 $W$ and $Z$ boson $p_{T}$ reweighting}

The $p_{T}$ distribution of the $W$ and $Z$ bosons is not well modeled in MC simulations. Therefore, a correction is derived from data using a measurement of the $Z \rightarrow$ ee differential cross section [97] which is applied to the $Z$ boson MC simulation [98]. As there is no such differential cross section measurement for the $W$ boson, in order to derive a similar correction, the product of the unfolded $Z / \gamma^{*}$ 
$p_{T}$ distribution and the ratio of $W$ to $Z$ cross sections at NLO is used to reweight the the $W$ boson $p_{T}$ distribution [99].

\subsubsection{Muon momentum resolution and scale}

Both the resolution and the scale of the muon momenta shows significant discrepancies between data and MC events [100]. It has been suggested that this discrepancy could be caused by, mismodeling of the muon hit efficiency, mismodeling of the hit resolution in simulation, mismodeling of the magnetic field, or from misalignment of the detector components [100]. To correct for these disagreements, a correction known as "oversmearing" is applied, where the curvature of the reconstructed muon momentum is smeared using either a single Gaussian or a double Gaussian function [101], as determined from $Z / \gamma^{*} \rightarrow \mu \mu$ and $J / \Psi \rightarrow \mu \mu$ data distributions. The distributions for both $Z / \gamma^{*} \rightarrow \mu \mu$ and $J / \Psi \rightarrow \mu \mu$ events before and after the "oversmearing" has been applied, are shown in Figure 7.1.

\subsubsection{Muon ID and track reconstruction}

The $\mathrm{D} \varnothing$ reconstruction software reconstructs a higher percentage of muons and their associated tracks for MC simulated events than for data events. An efficiency correction is applied to account for this. It is determined for each categorization of the muon; track quality, isolation quality, and identification quality in the muon system, as described in Section 4.3.1, and for the different data epochs. The efficiencies are determined as functions of various parameters, using the tag and probe method [57] from a sample of $Z \rightarrow \mu \mu$ events.

\section{Muon identification quality}

The variation of the muon reconstruction efficiencies with instantaneous luminosity, $L_{\text {inst }}$, and with $\eta, \phi$, and the $p_{T}$ of the muon is shown in Figures 7.2 and 7.3 

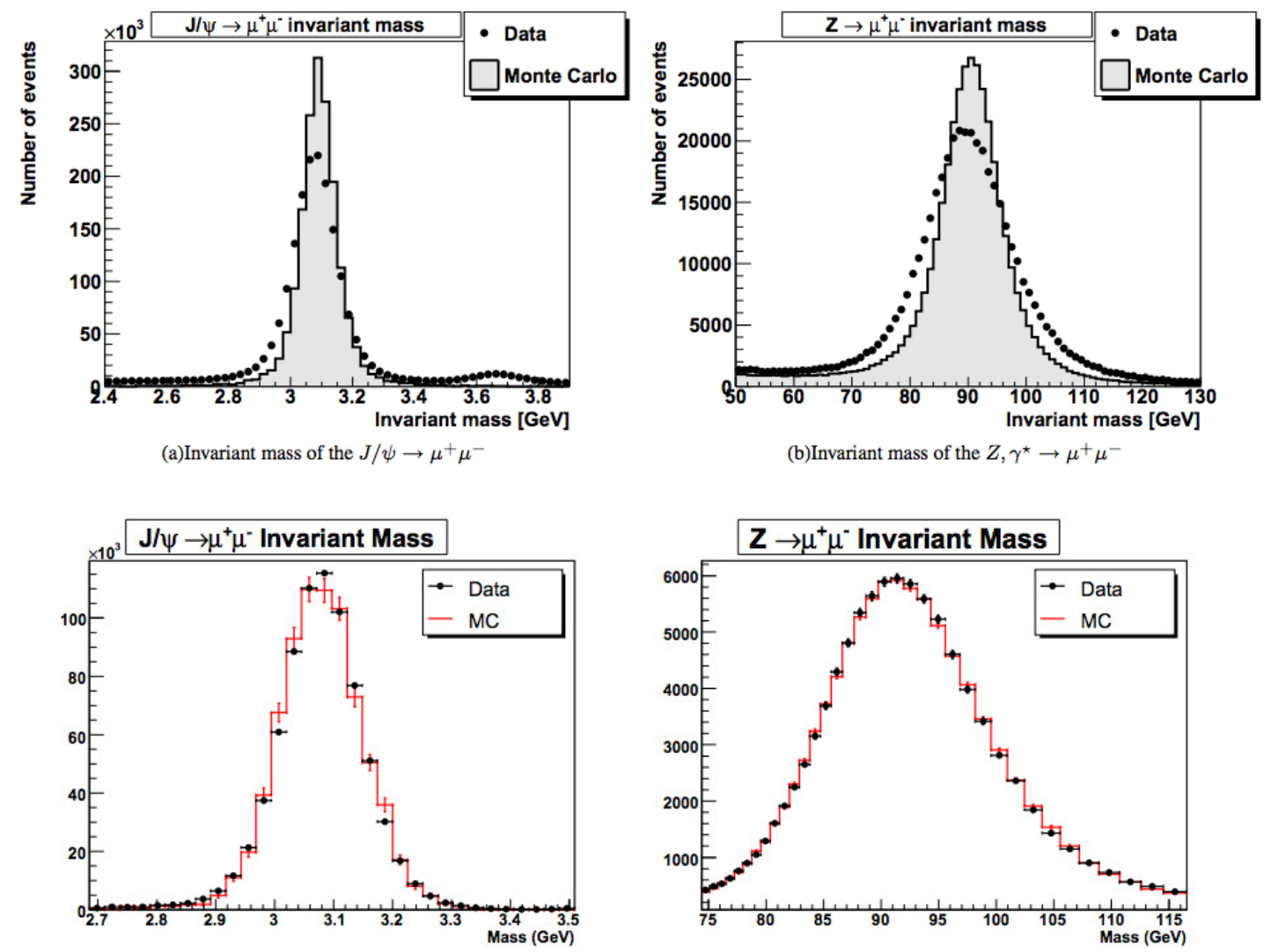

Figure 7.1: Comparison of data and MC simulations for $Z / \gamma^{*} \rightarrow \mu \mu$ (right) and $J / \Psi \rightarrow \mu \mu$ (left) events, with (bottom) and without (top) the muon oversmearing applied [100].

for the "Medium" and "MediumNseg3" quality requirements, respectively. The shapes seen are mainly due to the geometry of the detector, for example the lower efficiency between values of $0.5<|\eta|<0.1$, which corresponds to the transition region from the central to the forward regions of the detector [101]. The data and MC events show reasonable agreement for all variables studied. The efficiencies were studied separately for a muon with a $p_{T}$ greater than or less than $20 \mathrm{GeV}$ and were observed to be slightly higher when the muon has a $p_{T}>20 \mathrm{GeV}$. This effect is included as a systematic uncertainty.

The efficiencies are parametrized as a 2-dimensional function of $\eta$ and $\phi$, and 
are given for each data epoch, in Table 7.6. The efficiency is driven by the number of operating PDTs and the changes seen in its value are due to the aging of the detector or to recovered PDTs [101].
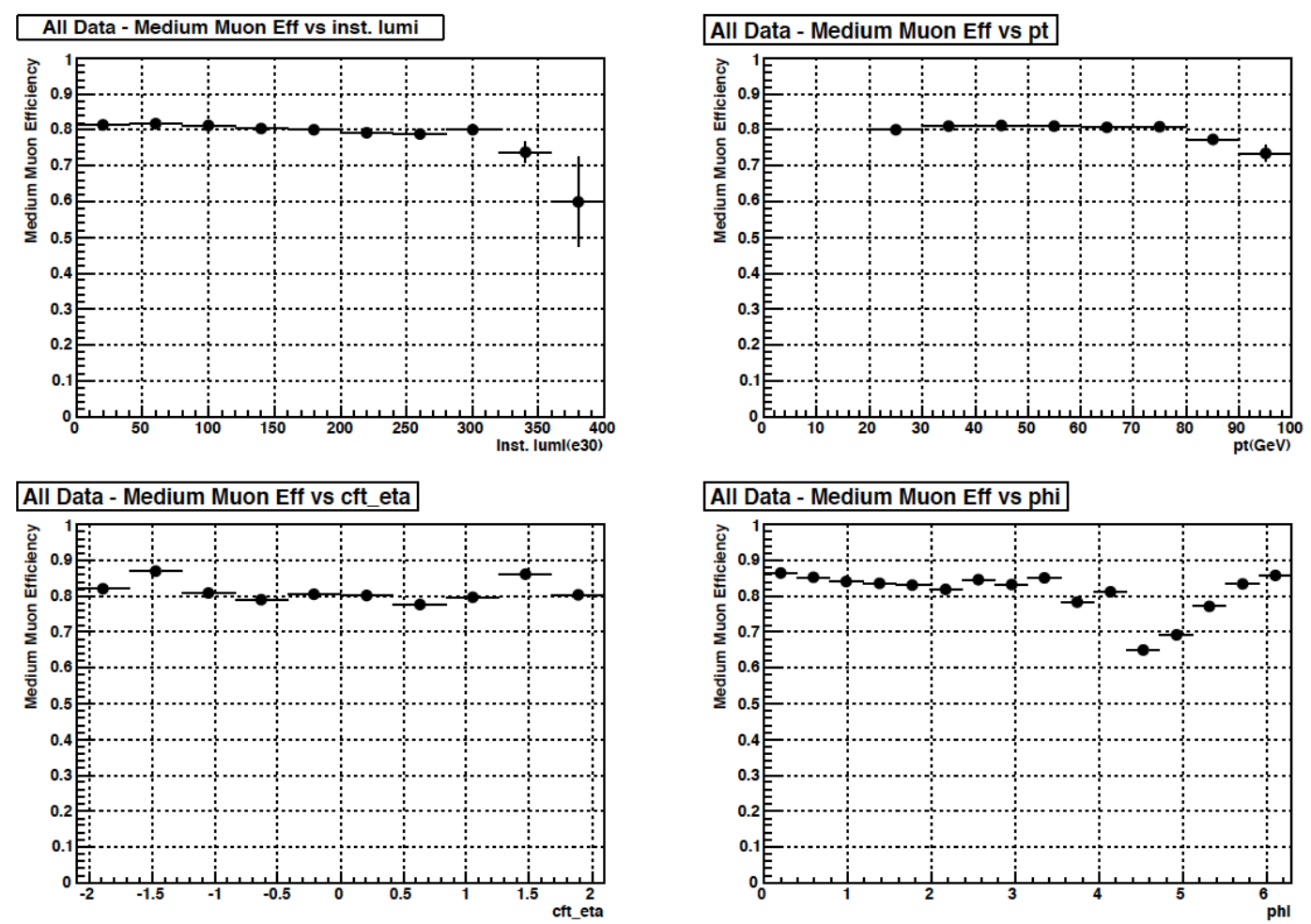

Figure 7.2: The muon reconstruction efficiencies as functions of $L_{i n s t}, \eta, \phi$, and the $p_{T}$ of the muon, for the "Medium" quality requirement [55].

\section{Track quality}

The track reconstruction efficiency for both data and MC events is shown as a function of $L_{\text {inst }}$ in Figure 7.4 and can be seen to fall as a function of the instantaneous luminosity. The efficiency is also seen to be dependent on $\eta$ and on the $p_{T}$ of the $Z$ boson. The track reconstruction efficiency is separated into two corrections, a "geometrical" efficiency parameterized in $\eta$ and the distance in $z$ and a luminosity based correction parametrized in $\eta$ and $L_{\text {inst }}$ [55]. 

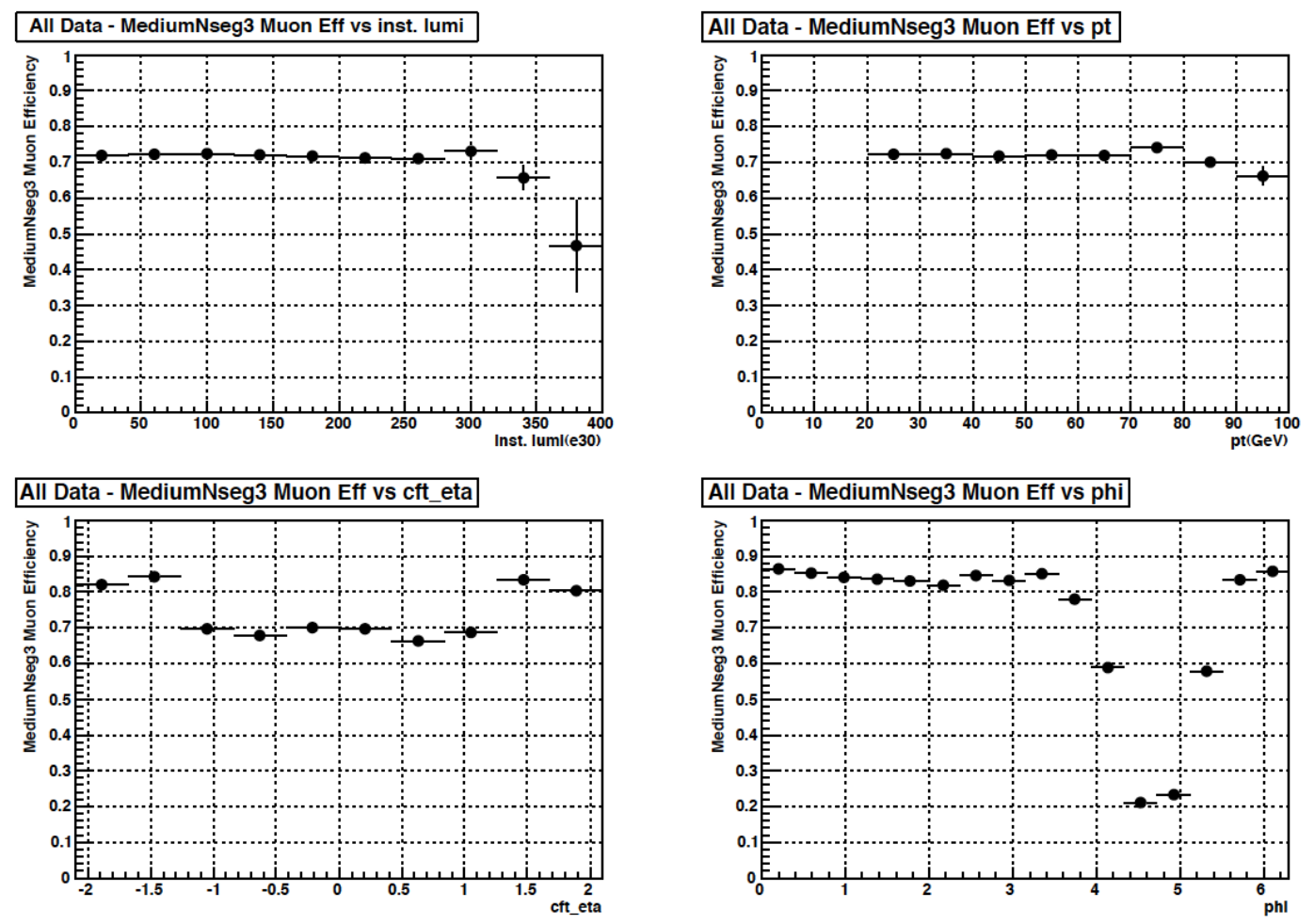

Figure 7.3: The muon reconstruction efficiencies as functions of $L_{\text {inst }}, \eta, \phi$, and the $p_{T}$ of the muon, for the "MediumNseg3" quality requirement [55].

The efficiencies are determined per track quality classification and per data epoch. Radiation damage in the detector leads to decreased efficiencies over the epochs and the improvement seen between Run IIb2 and Run IIb3 is due to the fixes to the SMT system between these epochs [101]. The determined efficiencies are listed for the TrackMedium quality in Table 7.6, for the different data taking epochs.

\section{Isolation quality}

The isolation efficiency is seen to be dependent on the $L_{i n s t}$, as it is strongly effected by pile up in the detector [101]. This is most noticeable for isolation quality definitions with the strongest isolation requirements, such as "NPTight" [101]. A difference in the reconstruction between data and MC samples for the $p_{T}$ of the 


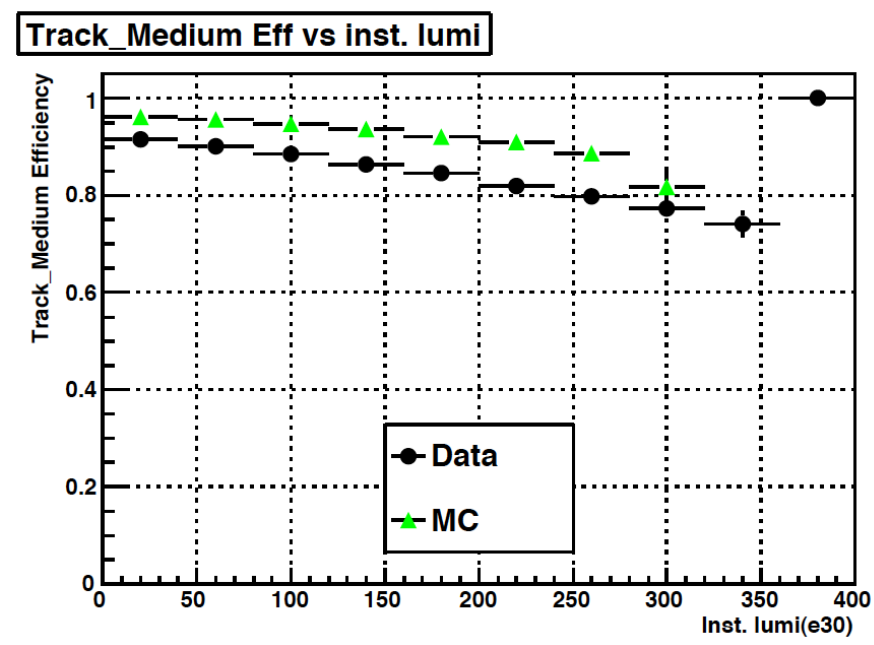

Figure 7.4: The track reconstruction efficiency for data and MC simulations for the "TrackMedium" quality requirement [55], as a function of the instantaneous luminosity.

muon is observed, and the efficiency has been shown to decrease when the muon is in the proximity of jets. The muon isolation correction is performed in stages, first a requirement of $\Delta R(\mu$, closest jet $)>0.5$ is placed. The efficiency of this correction is parametrized as a function of $L_{i n s t}$ and $\eta$. Next, the isolation quality is apply, the correction for which is parametrized in $|\eta| \times p_{T} \times \Delta R$ ( $\mu$,closest jet). The combined efficiencies for these two requirements, per data epoch, are given in Table 7.6 for the "NPTight" and "TopScaledLoose" isolation requirements.

\subsubsection{Tau lepton track identification}

The definitions used for quality of tau lepton tracks are equivalent to those defined for muon tracks. As there is no measurement of the track efficiency specially for the tau leptons, then the efficiency that is determined for muons is applied. The track efficiency for the tau lepton "tau_TrackLoose" requirement is shown in Table 7.6, per data taking epoch. This correction is applied identically as applied for the muons, separated into two corrections, a "geometrical" efficiency 


\begin{tabular}{l|c|cccc} 
& Quality & \multicolumn{4}{|c}{ Efficiency } \\
& requirement & Run IIb1 & Run IIb2 & Run IIb3 & Run IIb4 \\
\hline Muon & Medium & $81.54 \pm 0.14$ & $80.51 \pm 0.10$ & $80.85 \pm 0.16$ & $80.89 \pm 0.11$ \\
ID & MediumNeg3 & $72.29 \pm 0.16$ & $72.02 \pm 0.11$ & $71.66 \pm 0.18$ & $72.03 \pm 0.12$ \\
\hline Track & TrackMedium & $88.38 \pm 0.13$ & $86.57 \pm 0.10$ & $86.78 \pm 0.16$ & $84.09 \pm 0.12$ \\
\hline Isolation & NPTight & $90.06 \pm 0.11$ & $86.45 \pm 0.07$ & $87.44 \pm 0.11$ & $86.72 \pm 0.08$ \\
& TopScaledLoose & $98.81 \pm 0.04$ & $98.30 \pm 0.03$ & $98.58 \pm 0.04$ & $98.31 \pm 0.03$ \\
\hline \hline $\begin{array}{l}\tau \text { lepton } \\
\text { track }\end{array}$ & tau_TrackLoose & $92.21 \pm 0.11$ & $91.51 \pm 0.08$ & $92.37 \pm 0.12$ & $90.68 \pm 0.09$
\end{tabular}

Table 7.6: The measured efficiencies for the muon track quality, isolation quality, and identification quality in the muon system, for the different data epochs, as determined by Ref. [101]. The efficiency for the tau lepton track correction is also shown.

parameterized in $\eta$ and the distance in $z$, and a luminosity based correction parametrized in $\eta$ and $L_{\text {inst }}[55]$.

\subsection{6 $N N_{\tau}$ efficiency}

The quality of the reconstructed tau lepton candidate events is seen to be higher for MC samples than for data events, resulting in the $N N_{\tau}$ having a higher efficiency for separating MC tau leptons events from misreconstructed tau leptons than it does for data events. This results in shape discrepancies in the $N N_{\tau}$, with the data showing a wider peak at one and worse overall resolution [102]. When requiring that tau lepton candidates have a certain $N N_{\tau}$ value, the shape difference in the $N N_{\tau}$ introduces a difference in efficiency between the selection of MC and data events. The $N N_{\tau}$ therefore has a bin-by-bin reweighting applied which corrects the $N N_{\tau}$ shape for this discrepancy. This reweighting is determined separately for each of the three tau lepton types.

To determine this correction, the probability, $P$, for an event to be in a certain bin, $X$, is measured from a $Z / \gamma^{*} \rightarrow \tau \tau$ sample in both data and MC simulations [58]. These bin-by-bin probabilities for data, $P_{d a t a}^{Z / \gamma^{*} \rightarrow \tau \tau}(X)$, and MC events, 
$P_{M C}(X)$, are determined from,

$$
P_{M C}(X)=\frac{N_{M C}(X)}{N_{M C}}, \quad P_{\text {data }}^{Z / \gamma^{*} \rightarrow \tau \tau}(X)=\frac{N_{\text {data }}^{Z / \gamma^{*} \rightarrow \tau \tau}(X)}{N_{\text {data }}^{Z / \gamma^{*} \rightarrow \tau \tau}}
$$

where $N_{M C}(X)$ is the total number of predicted MC events for bin $X$ and $N_{M C}$ is the total number of $\mathrm{MC}$ events for all bins, $N_{\text {data }}^{Z / \gamma^{*} \rightarrow \tau \tau}(X)$ is the number of data events, for bin $X$, after all estimated MC background events, except $Z / \gamma^{*} \rightarrow \tau \tau$ events, are subtracted and $N_{d a t a}^{Z / \gamma^{*} \rightarrow \tau \tau}$ is the same integrated over all bins. The per bin correction, $f_{N N}(X)$, to the $N N_{\tau}$ is determined from the ratio of these probabilities [58],

$$
f_{N N}(X)=\frac{P_{M C}(X)}{P_{d a t a}^{Z / \gamma^{*} \rightarrow \tau \tau}(X)} .
$$

This correction is only derived for $N N_{\tau}$ values greater than 0.3 , as below that point the $N N_{\tau}$ is dominated by multijet events. Applying this reweighting induces an uncertainty on the efficiency of the $N N_{\tau}$, the value of which is dependent on the $N N_{\tau}$ requirement placed, the multijet estimation method used when performing this correction, and on the kinematics of the tau lepton candidates. The determined correction factors and the associated uncertainties per tau lepton Type, are given in Figure 7.5 for the $N N_{2010}$ and in Figure 7.6 for the $N N_{2012}$.

\subsubsection{Tau lepton energy scale}

The tau lepton energy scale has been shown to be different in data and MC simulations [102]. To correct for this a study is performed in tau lepton events selected from an enriched $Z \rightarrow \tau \tau$ sample [102]. It is assumed that the momentum of the tau leptons as determined from the tracks, $p_{T}^{\text {trk }}$, is well modeled and any mismodeling in the momentum as measured in the calorimeter, $p_{T}$, is due to the energy scale in the calorimeter not being well modeled. This correction can be probed by looking at the ratio of the tau lepton energy as measured in the 
Type-1
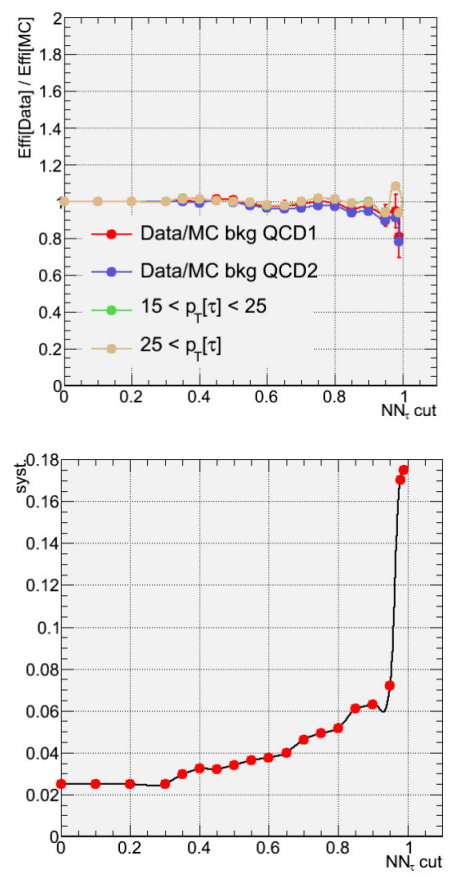

Type-2
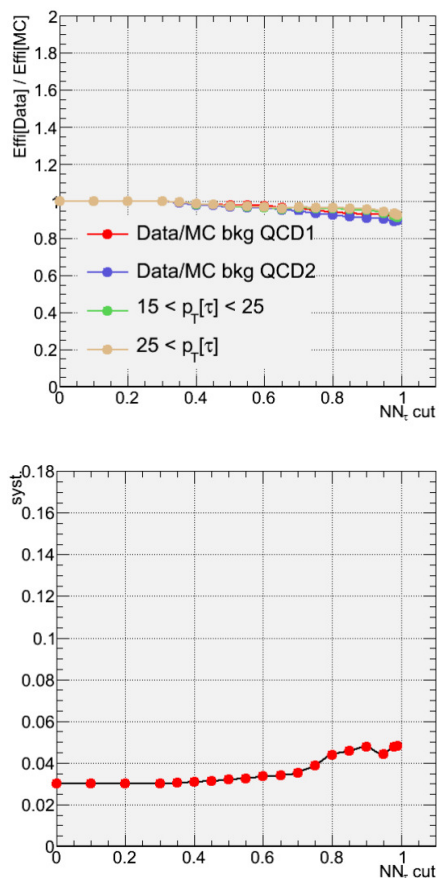

Type-3
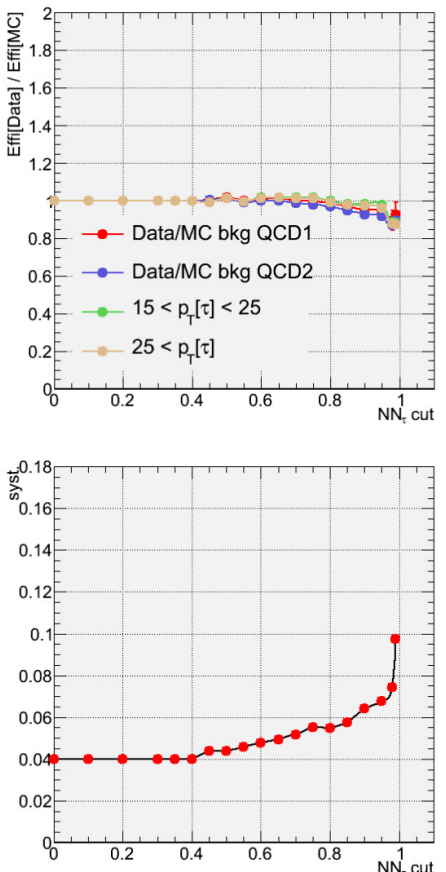

Figure 7.5: The tau lepton NN efficiency corrections (top) and associated total systematic uncertainty (bottom) as a function of the $N N_{\tau}$, for $N N_{2010}$, shown per tau lepton type [103]. These efficiencies have been determined for a sample of combined Run IIb 2 - 4 data.

calorimeter, $E_{T}^{c a l}$, to the track momentum, $p_{T}^{\text {trk }}$, in both data and MC samples and any deviation from one being assumed to arise from mismodeling of the energy scale in the calorimeter. The correction is determined on a bin-by-bin basis, per tau lepton type, parametrized as a function of the $F_{E M}$, the ratio of the energy cluster corresponding to a tau lepton in the EM layers of the calorimeter, to the total energy in the calorimeter. The determined correction for the three tau lepton types is shown in Figure 7.7. 
Type-1
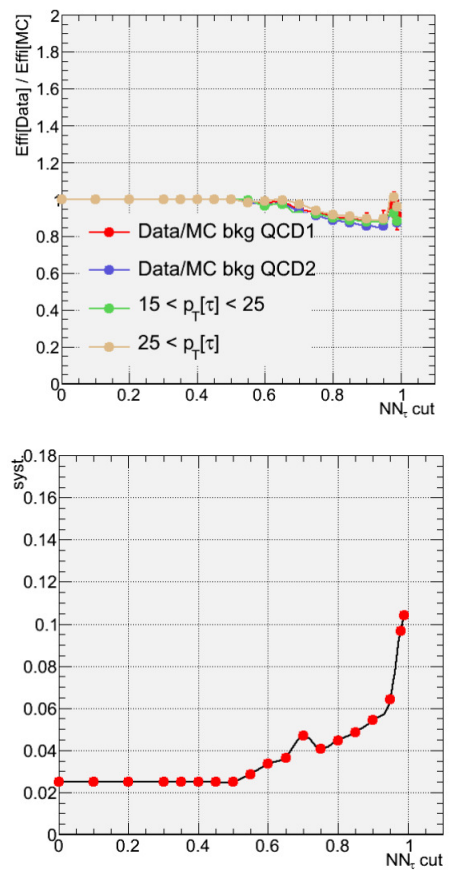

Type-2
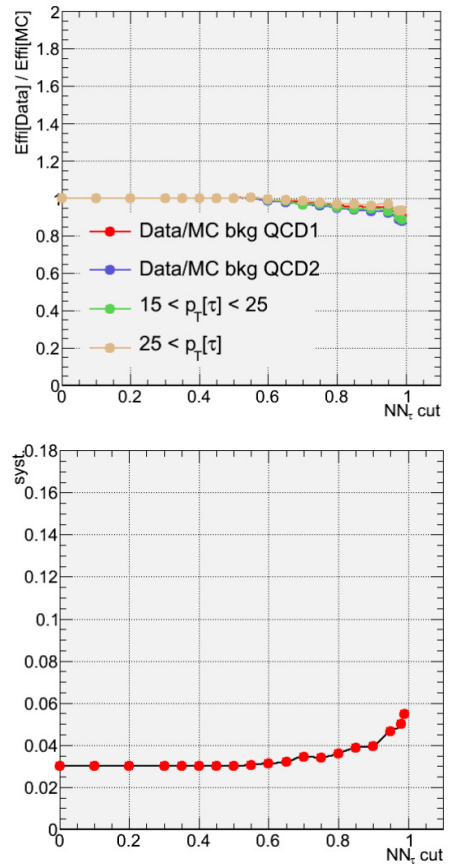

Type-3
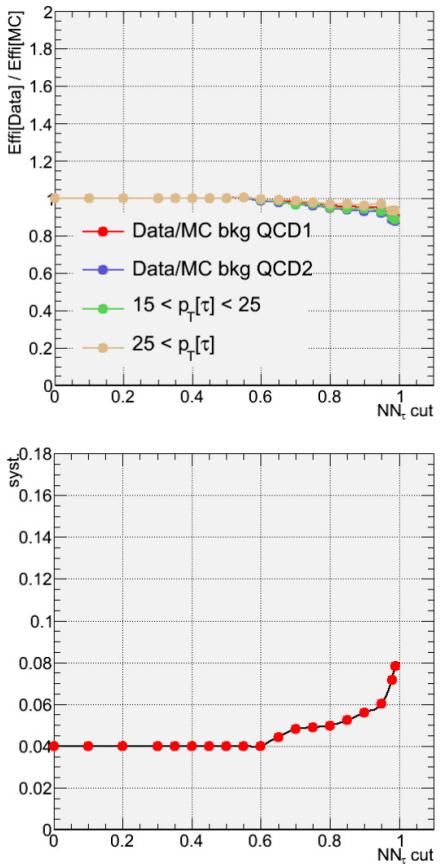

Figure 7.6: The tau lepton NN efficiency corrections (top) and associated total systematic uncertainty (bottom) as a function of the $N N_{\tau}$, for $N N_{2012}$, shown per tau lepton type [103]. These efficiencies have been determined for a sample of combined Run IIb 2 - 4 data.

\subsection{Selection requirements}

Both the SM Higgs boson and the doubly charged Higgs boson searches, require that there are at least two hadronically decaying tau leptons and one muon in the selected events, where the two highest $p_{T}$ tau leptons, $\tau_{1}$ and $\tau_{2}$, and the highest $p_{T}$ muon are selected. To remove low quality, poorly reconstructed tau leptons and muons and to ensure that all determined $\mathrm{MC}$ corrections are applicable, certain selection requirements are applied to both searches. These corrections are applied at a stage known as "pre-selection" and will be described in this section. Additional analysis specific corrections, known as "final selection", designed to isolate the signal events are applied, after the agreement between the $\mathrm{MC}$ samples 

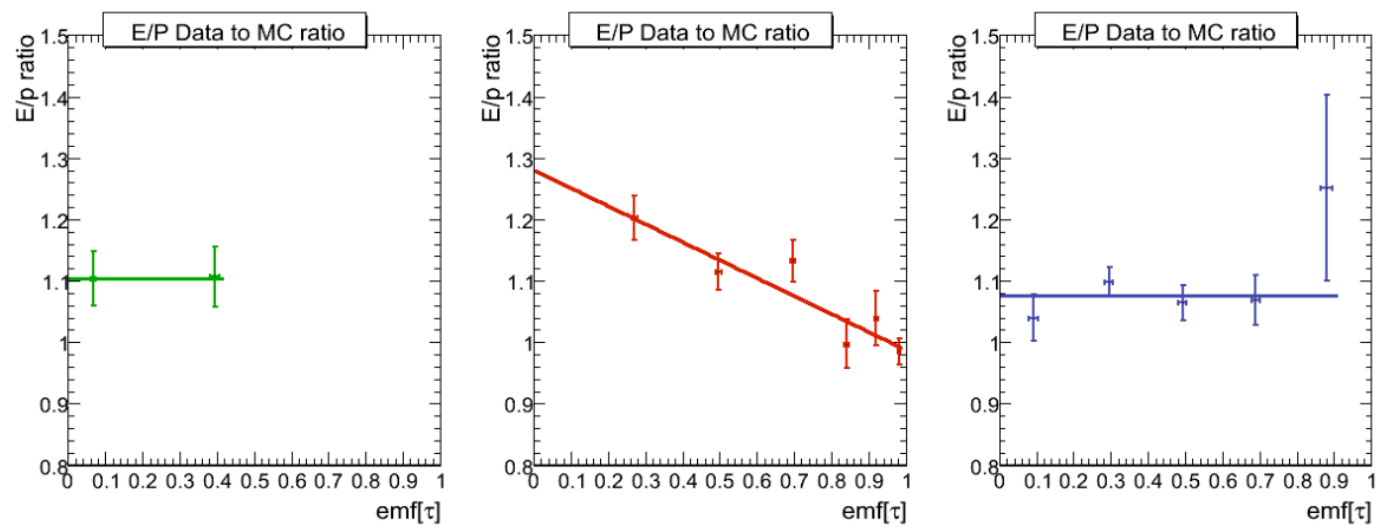

Figure 7.7: The ratio of $E_{T}^{c a l}$ to the $p_{T}^{\text {trk }}$ for the three tau lepton types as a function of the EM fraction [104]. Shown for Type-1 tau lepton candidates (left), Type-2 tau lepton candidates (middle) and Type-3 tau lepton candidates (right).

and the observed data has been checked.

\subsubsection{Muon pre-selection}

At least one muon is selected and, at this stage, no requirements are placed on additional muons in the event. These muons are reconstructed from data following the method covered in Section 4.3.1. Basic requirements are placed on the muons at skimming level, as described in Section 7.1.1. At pre-selection certain additional requirements must be applied in order for the SignalMuonOR trigger efficiency corrections to be applicable [55].

- The muons must have $p_{\mathrm{T}}>15 \mathrm{GeV}$.

- Muons must be reconstructed in the range $|\eta|<1.6$, which guarantees that muons are reconstructed within the muon detector coverage.

The selected muons must also pass three quality requirements; for their isolation, track, and identification quality in the muon system as described in Section 4.3.1. These requirements are analysis specific and will be described in 
Section 8.4.2 and Section 9.6.1 for the two searches performed.

\subsubsection{Hadronically decaying tau lepton pre-selection}

At skimming level (see Section 7.1.1) the tau lepton candidates are required to pass basic requirements. The following additional constraints are required at pre-selection level for all tau lepton candidates [105].

- The tau lepton candidates are required to be within the detector region of $|\eta|<2.0$.

- The tau lepton candidates are required to have $p_{T}>12.5 \mathrm{GeV}$ for Type-1 and Type-2 tau leptons, and $p_{T}>15 \mathrm{GeV}$ for Type-3.

- The tau lepton tracks are required to satisfy the "tau_TrackLoose" quality criteria, as described in Section 4.3.1.

- The tau lepton candidates are required to be isolated from reconstructed muons, $\Delta R\left(\tau_{i}, \mu_{j}\right)>0.4$, to remove muons that have been misidentified as tau leptons. All objects that pass the "Loose" muon identification quality requirement and are in this cone are removed.

- The ratio of the calorimeter energy to the track momentum, $E_{T}^{c a l} / p_{T}^{t r k}>$ 0.65/0.5/0.5, for tau lepton type Type-1/Type-2/Type-3, which removes contamination from cosmic ray muons [102].

- To further remove contamination from muons that are misreconstructed as single track tau leptons, Type- 1 tau leptons are required to have $R_{\tau}=\left(F_{H F}+\right.$ $\left.F_{E M}\right) E_{T}^{\tau} / p_{T}^{\text {trk }}>0.3$, where $F_{H F}$ is the fine hadronic calorimeter fraction and $F_{E M}$ is the electromagnetic fraction [61]. 


\subsection{Misreconstructed tau lepton events}

When requiring two hadronically decaying tau leptons large portions of the MC background events that are selected will involve cases where a jet is misidentified as a hadronically decaying tau lepton. As these events are known to contain a misreconstructed object it is necessary to perform a study to check that they are modeled well by the MC simulations. To perform this study a sample was selected with two medium quality hadronic tau leptons and two muons. In this sample the misreconstructed events are expected to originate from $Z / \gamma^{*} \rightarrow \mu \mu$ events with additional jets in the events, $Z / \gamma^{*}(\rightarrow \mu \mu)+$ jets, where the jets are misreconstructed as tau leptons. Therefore in comparing the simulation of these events to the observed data, the level of mismodeling of these misreconstructed tau lepton events can be determined.

To produce the sample for this study, the following requirements are implemented in addition to the pre-selection requirements:

1. Number of muons $\geq 2$,

2. A requirement on $\mathrm{NN}_{\tau}>0.3$,

3. A requirement that $\left|\eta_{\tau}\right|<1.5$.

The distributions of the $p_{T}$ and $\eta$ of the two highest $p_{T}$ tau leptons and the muon, for the observed data and predicted MC events for this sample are shown in Figure 7.8. The corresponding numbers of events from MC simulations and observed in data events are listed in Table 7.7.

From Figure 7.8 and Table 7.7, it can be seen that as expected, the majority of the events $(>90 \%)$ are from the $Z / \gamma^{*}(\rightarrow \mu \mu)+$ jet sample, with small contributions from $Z / \gamma^{*}(\rightarrow \tau \tau)+$ jet, $t \bar{t}$, and diboson samples. Good agreement is seen both in the shape and in the number of the observed data and predicted MC events within the statistics available. This leads to the assumption that these 

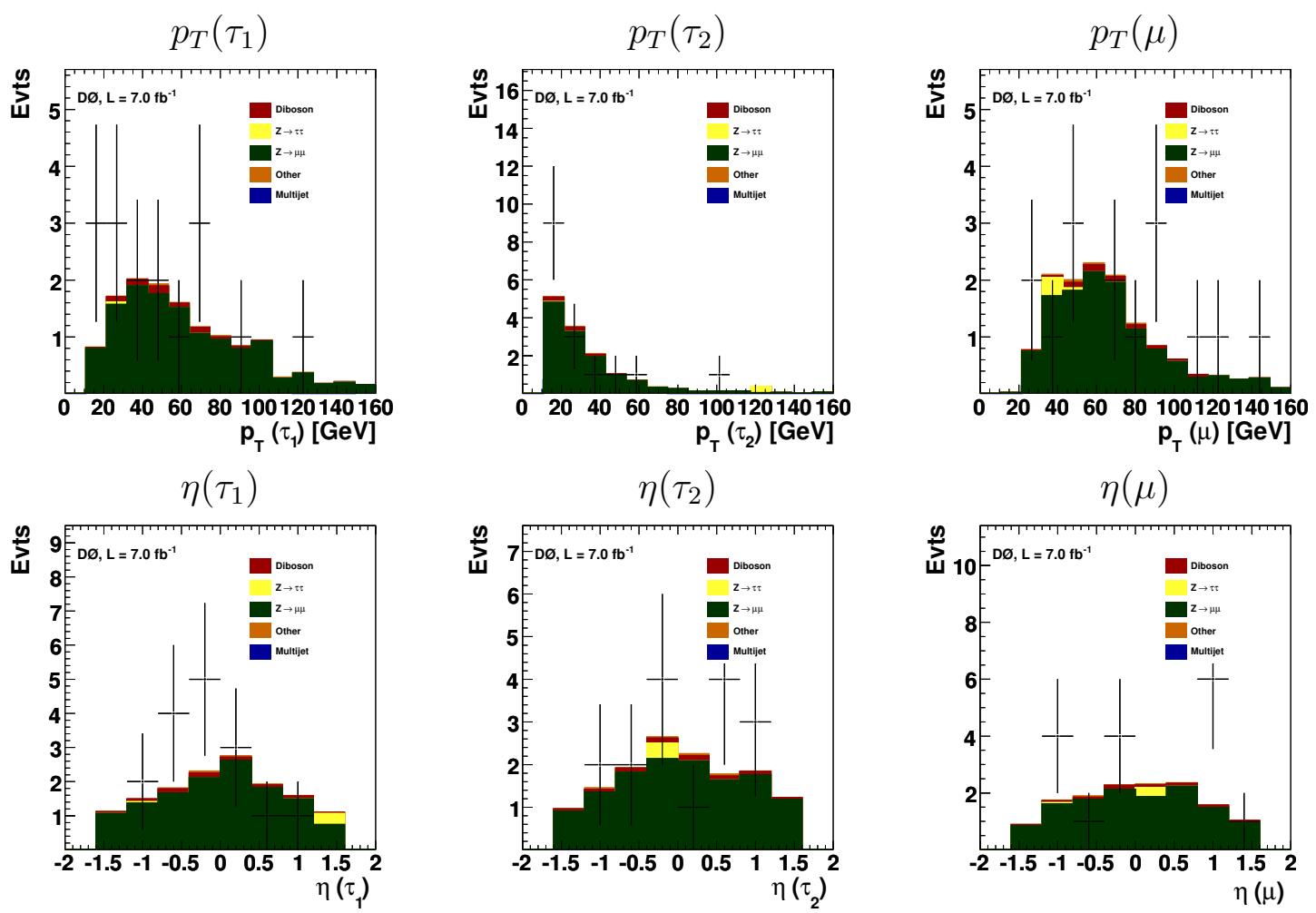

Figure 7.8: Data distributions compared to the predicted MC events for the study into misreconstructed tau leptons. The 'Other' background contains the contribution from $Z \rightarrow e e, t \bar{t}$, and $W+$ jets.

misreconstructed events where jets are misidentified as a hadronically decaying tau leptons will be modeled well by the MC simulation within the statistics available at the final selection. This study does not look into the modeling of $W+$ jet events which is covered in Section 7.7.

\section{$7.7 W+$ jets normalization}

$W+$ jet events are seen to be not well modeled in tau lepton and muon final states at $\mathrm{D} \varnothing[106,107]$, therefore a correction is derived in which the $W+$ jet MC events are normalized to what is observed in the data. This normalization is expected to be dependent on tau lepton type and on the electric charge requirements on the 


\begin{tabular}{l|c} 
Data & 16 \\
\hline$Z \rightarrow \tau \tau$ & $0.36 \pm 0.05$ \\
$Z \rightarrow \mu \mu$ & $12.89 \pm 1.64$ \\
$Z \rightarrow e e$ & $<0.01$ \\
$t \bar{t}$ & $0.20 \pm 0.03$ \\
Diboson & $0.67 \pm 0.09$ \\
$W+$ jet & $<0.01$ \\
multijet & $<0.01$ \\
\hline Total Bkg & $14.13 \pm 1.81$
\end{tabular}

Table 7.7: The observed number of data events compared to predictions from MC simulations for the misreconstructed tau lepton study.

three final state objects. As tau leptons are more likely to be misreconstructed than muons, then a sample requiring that tau leptons have the opposite electric charge (the tau lepton and muon have the same electric charge) will have a higher contribution from misreconstructed tau leptons, then a sample where the two tau lepton have the same electric charge. These samples are referred to as same-sign (SS) and opposite-sign (OS), respectively. Therefore the $W+$ jet event normalization is determined separately for both SS and OS events and per tau lepton type.

To determine the normalization of the $W+$ jet events, the shape of the $W+$ jet event distributions is taken from ALPGEN + PYTHIA MC samples, as listed in Table 7.4, and the normalization is determined from a sample of $W$ boson enriched data events. For this enriched sample additional requirements are implemented on top of the pre-selection requirements, the transverse mass of $M_{T}>40$ and $p_{T}$ of the highest $p_{T}$ tau lepton $>20 \mathrm{GeV}$ to remove multijet contamination. This enriched sample is referred to as " $W$-rich" and is assumed to have a negligible contribution from multijet events. To determine the normalization of the $W+$ jet MC sample a scale factor, $R_{W}$, is defined, where the value $R_{W}$ can be determined from, 


$$
R_{W}=\frac{N^{\mathrm{W}-\text { rich }}(\text { data })-N^{\mathrm{W}-\text { rich }}(\mathrm{MC})}{N^{\mathrm{W}-\text { rich }}(\mathrm{W}+\text { jet } \mathrm{MC})}
$$

where $N^{\mathrm{W}-\text { rich }}$ (data) is the number of data events in the $W$-rich region, $N^{\mathrm{W}-\text { rich }}(\mathrm{MC})$ is the number of MC events, not including $W+$ jet $\mathrm{MC}$ events, in the $W$-rich region and $N^{\mathrm{W}-\text { rich }}(W+$ jet $\mathrm{MC})$ is the number of $W+$ jet $\mathrm{MC}$ events in the $W$-rich region. The determined values of $R_{W}$ are listed in Table 7.8 for both the SS and OS sample and per tau lepton type. The distributions of the background samples compared to observed data before and after this reweighting are shown in Figure 7.9. In these plots no contribution from the multijet background is included, as its contribution is determined after this correction has been applied. Hence the observable discrepancy at low $p_{T}$ values where these events will have a large contribution. One should concentrate on the tail of the distributions which can be thought of as the region that is being fitted.

\begin{tabular}{c|cc} 
Tau lepton Type & \multicolumn{2}{|c}{$R_{W}$ value } \\
\hline & OS & SS \\
\hline Type-1 & $0.82 \pm 0.02$ & $1.14 \pm 0.03$ \\
Type-2 & $0.63 \pm 0.02$ & $1.12 \pm 0.03$ \\
Type-3 & $0.52 \pm 0.02$ & $0.55 \pm 0.02$
\end{tabular}

Table 7.8: The determined values of the $W+$ jets reweighting factor, $R_{W}$, for the different tau lepton types and the same-sign (SS) and opposite-sign (OS) samples.

\subsection{Instrumental background estimation}

For an event selection with two hadronically tau leptons there will be a contribution to data from instrumental backgrounds in the detector, commonly referred to as multijet events. These arise when two jets in the detector are misidentified as two hadronically decaying tau leptons and a muon is either also misreconstructed or fails the isolation requirements from a heavy quark decay. This contribution 

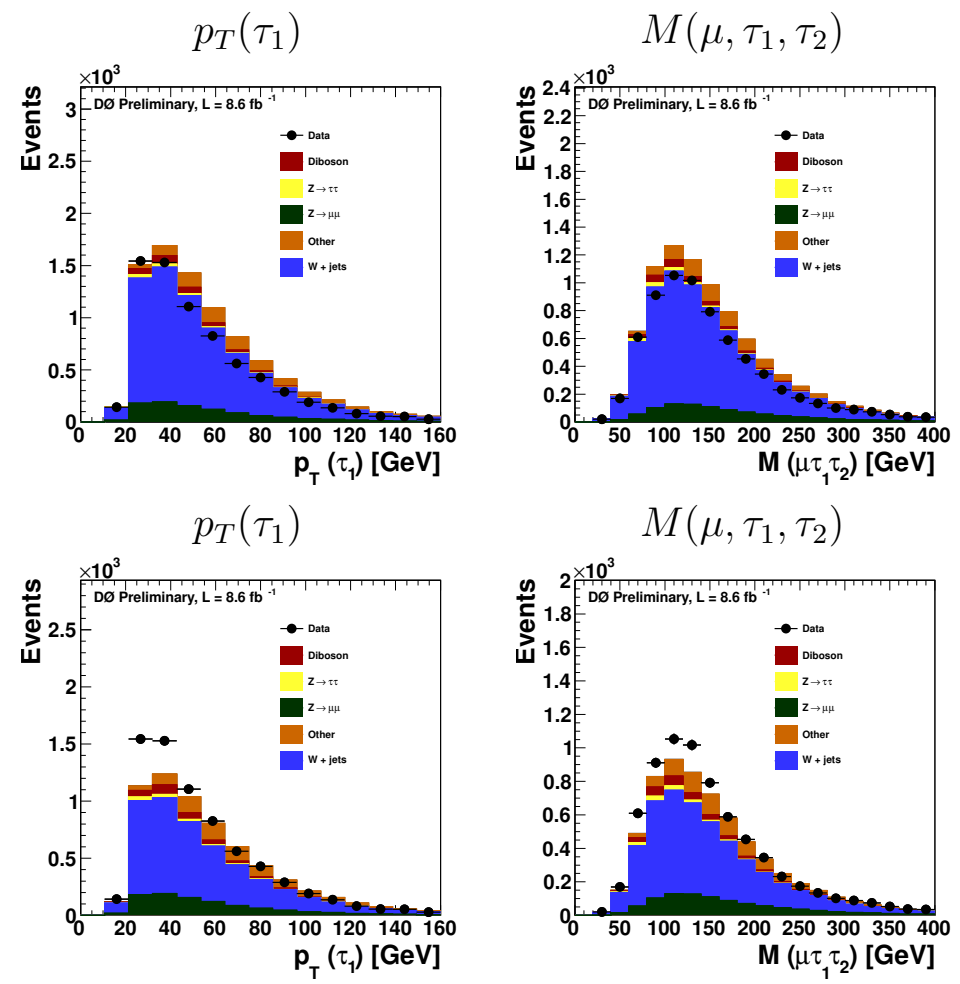

Figure 7.9: Data distributions for the $W$-rich selection compared to the sum of the expected background from MC simulations at this selection, for before (top) and after (bottom) the $W+$ jets reweighting is applied. The 'Other' background sample contains the contributions from $Z / \gamma^{*} \rightarrow e e$ and $t \bar{t}$ events. No multijet contribution is included in these plots.

to the background events is hard to model with MC simulations and hence is determined from data.

Two methods for estimating these background events have been derived. Method 1 is based on the $N N_{\tau}$ of the two highest $p_{T}$ tau leptons and is described in Section 7.8.1. A second method acts as a cross check and is based on the charge of the three reconstructed objects and the highest $p_{T} N N_{\tau}$, this is described in Section 7.8.2. Both methods can be applied to both the $N N_{2010}$ and the $N N_{2012}$. 


\subsubsection{Instrumental background Method 1}

The method to estimate the instrumental background contribtion relies on selecting four orthogonal regions determined by the value of the $N N_{\tau}$ for the two highest $p_{T}$ tau lepton candidates, where $N N_{\tau}^{1}$ is the highest $p_{T}$ tau lepton Neural Network and $N N_{\tau}^{2}$ is the second highest $p_{T}$ tau lepton Neural Network. These regions are defined by two $N N_{\tau}$ requirements, one tight requirement, $N N_{\tau}^{\text {tight }}$, on the upper value of the $N N_{\tau}$ and one loose requirement on the lower value of the $N N_{\tau}, N N_{\tau}^{\text {loose }}$. These four regions are represented diagrammatically in Figure 7.10 .

The sample that the multijet background contribution is being determined for, is referred to as the TTNN sample, as there is a tight $N N_{\tau}$ requirement on both tau leptons. This is generally the final selection sample, as described in Sections 8.5.1 and 9.6.4. Three orthogonal regions are also determined, one to derive the shape of the multijet contribution and two to normalize it. Other than the requirements that are being varied for the estimation, the $N N_{\tau}$ requirements, all other requirements should be kept the same for the four regions.

\section{- Tight-Tight $\mathrm{NN}_{\tau}(\mathrm{TTNN})$ :}

$N N_{\tau}^{1}>N N_{\tau}^{t i g h t}$ and $N N_{\tau}^{2}>N N_{\tau}^{t i g h t}$

The sample the multijet background contribution is being determined for, with a tight $N N_{\tau}$ requirement on both tau leptons.

\section{- Loose-Loose $\mathrm{NN}_{\tau}$ (LLNN):}

$N N_{\tau}^{1} \leq N N_{\tau}^{\text {tight }}, N N_{\tau}^{1}>N N_{\tau}^{\text {loose }}$ and $N N_{\tau}^{2} \leq N N_{\tau}^{\text {tight }}, N N_{\tau}^{2}>N N_{\tau}^{\text {loose }}$

This region is composed primarily of instrumental background events. It is used to determine the shape of instrumental background events in the TTNN region.

- Loose-Tight $\mathrm{NN}_{\tau}($ LTNN): 


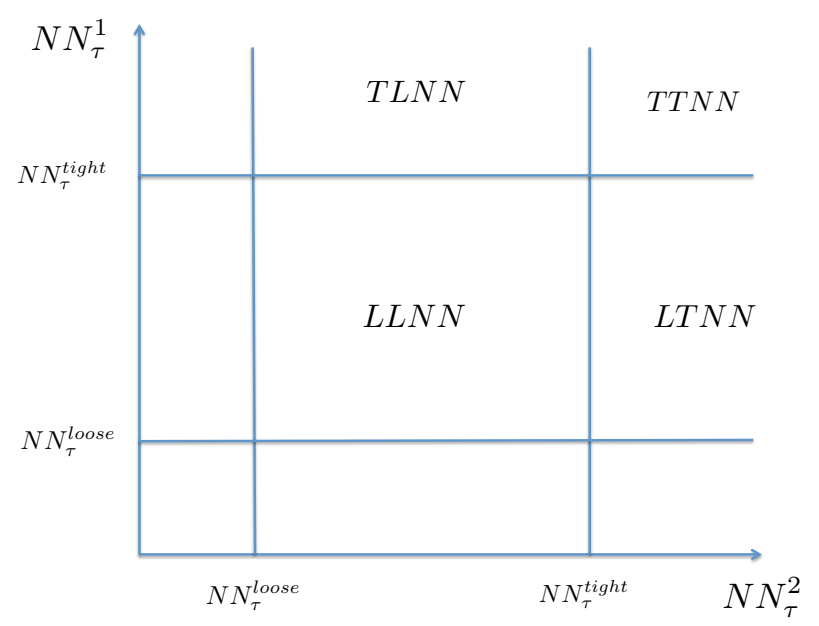

Figure 7.10: Diagrammatic representation of the four $N N_{\tau}$ regions defined for the instrumental background Method 1, TTNN, TLNN, LTNN, and LLNN. Showing the requirements for the highest $p_{T}$ tau lepton $N N_{\tau}$ on the $y$-axis and for the second highest $p_{T}$ lepton $N N_{\tau}$ on the $x$-axis.

$N N_{\tau}^{1} \leq N N_{\tau}^{\text {tight }}, N N_{\tau}^{1}>N N_{\tau}^{\text {loose }}$ and $N N_{\tau}^{2}>N N_{\tau}^{\text {tight }}$

An orthogonal sample where one of the two tau lepton candidates has a tight

$N N_{\tau}$ requirement and one is required to fail the tight $N N_{\tau}$ requirement.

\section{- Tight-Loose $\mathrm{NN}_{\tau}$ (TLNN):}

$N N_{\tau}^{1}>N N_{\tau}^{\text {tight }}$ and $N N_{\tau}^{2} \leq N N_{\tau}^{\text {tight }}, N N_{\tau}^{2}>N N_{\tau}^{\text {loose }}$

A second orthogonal sample where the $N N_{\tau}$ requirements on the two tau leptons are reversed compared to LTNN.

The shape of the instrumental background contribution is taken from the LLNN sample, which is normalized to be applicable in the TTNN region by a scale factor derived from the relative efficiencies of the LTNN and TLNN samples. The efficiency for events to be selected by the LTNN sample, $\epsilon_{L T}$, is determined from,

$$
\epsilon_{L T}=\frac{N_{L T N N}^{\text {data }}-N_{L T N N}^{M C}}{N_{L L N N}^{\text {data }}-N_{L L N N}^{M C}},
$$


where $N_{L T N N}^{d a t a}$ is the number of observed data events and $N_{L T N N}^{M C}$ the total number of predicted background MC events, as determined from the samples listed in Tables 7.3 and 7.4, in the orthogonal LTNN sample. Similarly $N_{L L N N}^{d a t a}$ and $N_{L L N N}^{M C}$ are the number of observed data events and total predicted background $\mathrm{MC}$ events in the LLNN sample. The efficiency for events to be selected by the TLNN sample, $\epsilon_{T L}$, is determined by

$$
\epsilon_{T L}=\frac{N_{T L N N}^{\text {data }}-N_{T L N N}^{M C}}{N_{L L N N}^{\text {data }}-N_{L L N N}^{M C}},
$$

where $N_{T L N N}^{\text {data }}$ and $N_{T L N N}^{M C}$ are the numbers of data and background MC events observed in the in the TLNN sample. The number of instrumental background events in the TTNN, $N_{T T N N}^{I B}$, can now be determined from,

$$
N_{T T N N}^{I B}=\epsilon_{L T} \epsilon_{T L}\left(N_{L L N N}^{\text {data }}-N_{L L N N}^{M C}\right)
$$

\subsubsection{Instrumental background Method 2}

The cross check method used to determine instrumental multijet background contribution relies on selecting four orthogonal regions determined by the value of the $N N_{\tau}$ output for the highest $p_{T}$ tau lepton, $N N_{\tau}^{1}$, and on the absolute sum of the electric charge of the final state particles, $|Q|=\left|q_{\tau_{1}}+q_{\tau_{2}}+q_{\mu}\right|$. The multijet background is determined for the region referred to as SR, generally the final selection sample as described in Sections 8.5.1 and 9.6.4, and three orthogonal regions are determined, one to derive the shape of the multijet contribution and two to normalize it. Other than the requirements that are being varied for the estimation, $N N_{\tau}^{1}$ and $|Q|$, all other requirements should be kept the same for the four regions.

For signal events it is expected that two of the three reconstructed final state particles will have the same electric charge, for example in $H W^{+} \rightarrow \tau^{+} \tau^{-} \mu^{+} \nu_{\mu}$ or $H^{++} H^{--} \rightarrow \tau^{+} \tau^{+} \tau^{-} \tau^{-}$. For instrumental background events it is as likely for all the three selected particles to have the same charge, as it is for two of them. 
This can be seen in Figure 7.11 which shows the sum of the electric charges of the two selected tau leptons and the muon, $Q$. It can be seen that the signal events are nearly all in the $|Q|=1$ bin, with the $|Q|=3$ being mainly occupied by the instrumental multijet background events. The "charge flip" event rate, where leptons are reconstructed with the wrong charge, has been shown to be very small of the order of $10^{-4}$ [108]. Using this knowledge four regions are defined.

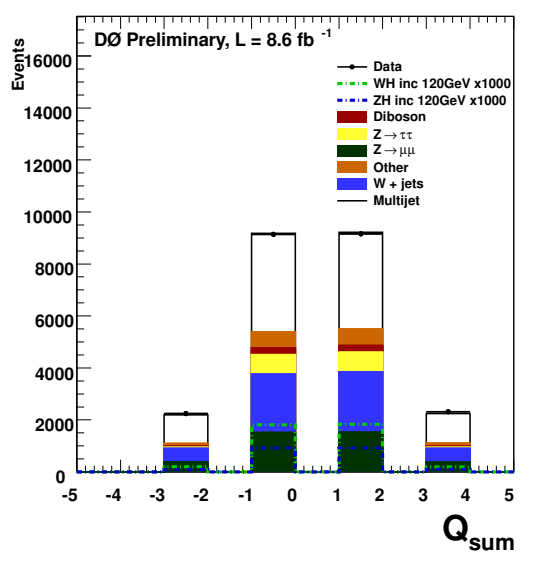

Figure 7.11: The sum of the electric charge of the three selected particles, $Q=q_{\tau_{1}}+q_{\tau_{2}}+q_{\mu}$, at pre-selection. The 'Other' background sample contains the contributions from $Z \rightarrow e e$ and $t \bar{t}$ events. The multijet was determined using Method 1 described in Section 7.8.1 and the $W+$ jets contribution is reweighted from MC as described in Section 7.7.

- Signal Region (SR):

$N N_{\tau}^{1}>N N_{\tau}^{\text {tight }}$ and $|Q|=1$

The region the multijet contribution is being determined for.

- Loose Region (LR):

$N N_{\tau}^{1}<N N_{\tau}^{\text {tight }}, N N_{\tau}^{1}>N N_{\tau}^{\text {loose }}$ and $|Q|=3$

The LR region is composed primarily of multijet events. It is used to derive the shape of multijet events in the SR sample.

- Normalization Region 1 (NR1): 


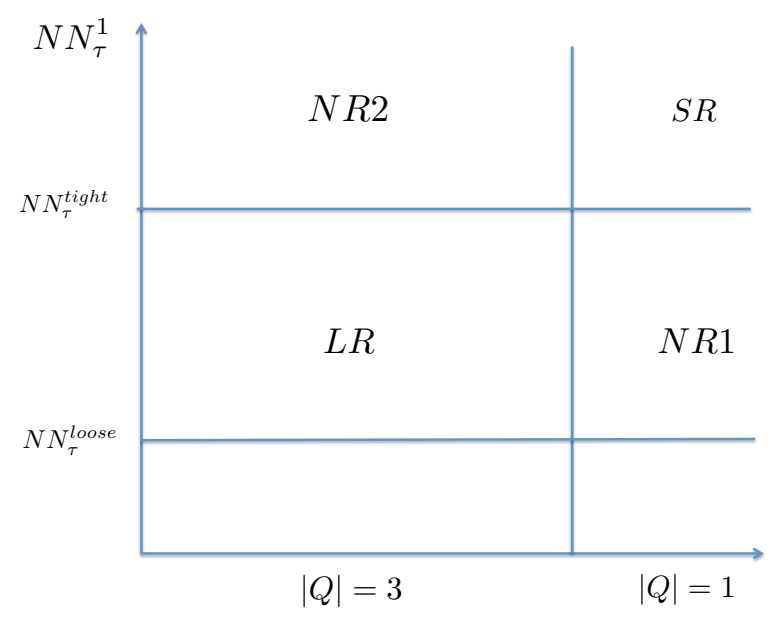

Figure 7.12: Diagrammatic representation of the four regions defined for the instrumental background Method 2, SR, NR1, NR2 and LR. Showing the requirements for the $N N_{\tau}$ of the highest $p_{T}$ tau leptons on the $y$-axis and the electric charge requirement, $|Q|$, on the $x$-axis.

$$
N N_{\tau}^{1}<N N_{\tau}^{\text {tight }}, N N_{\tau}^{1}>N N_{\tau}^{\text {loose }} \text { and }|Q|=1
$$

An orthogonal sample to the SR sample, though it has the same electric charge requirement the reversed $N N_{\tau}$ requirement assures that these events do not occur in any other regions.

\section{- Normalization Region 2 (NR2):}

$$
N N_{\tau}^{1}>N N_{\tau}^{\text {tight }} \text { and }|Q|=3
$$

A second orthogonal sample to the SR sample, though it has the tight $N N_{\tau}$ requirement, the requirement on $|Q|=3$ assures that these events are not selected in any of the other samples.

The four orthogonal regions are demonstrated diagrammatically in Figure 7.12. For this method, the shape for the instrumental background contribution is taken from the LR sample. The multijet sample is normalized to the SR region using a scale factor derived from the relative efficiencies of the NR1 and NR2 samples. The efficiency for events to be selected by the NR1 sample, $\epsilon_{N R 1}$, is determined 
from,

$$
\epsilon_{N R 1}=\frac{N_{N R 1}^{\text {data }}-N_{N R 1}^{M C}}{N_{L R}^{\text {data }}-N_{L R}^{M C}},
$$

where $N_{N R 1}^{\text {data }}$ is the number of observed data events and $N_{N R 1}^{M C}$ is the total number of background MC events, in the NR1 sample. $N_{L R}^{\text {data }}$ is the number of data events and $N_{L R}^{M C}$ the total number of predicted background MC events in the LR sample. The efficiency for events to be selected by the NR2 sample, $\epsilon_{N R 2}$, is determined from,

$$
\epsilon_{N R 2}=\frac{N_{N R 2}^{\text {data }}-N_{N R 2}^{M C}}{N_{L R}^{\text {data }}-N_{L R}^{M C}},
$$

where $N_{N R 2}^{\text {data }}$ is the number of observed data and $N_{N R 2}^{M C}$ the number of predicted background MC events, in the NR2 sample. The number of instrumental background events in the SR sample, $N_{S R}^{I B}$, can then be determined from,

$$
N_{S R}^{I B}=\epsilon_{N R 1} \epsilon_{N R 2}\left(N_{L R}^{\text {data }}-N_{L R}^{M C}\right) .
$$

\subsubsection{Application of instrumental background methods}

As indicated in Equations 7.4 to 7.6, and 7.7 to 7.9 all respective backgrounds, other than instrumental background events being estimated, are subtracted from the data sample using the corresponding MC background samples listed in Tables 7.3 and 7.4 after all reweightings and MC event corrections applied. These background are $W+$ jet, $Z / \gamma^{*} \rightarrow \tau \tau, Z / \gamma^{*} \rightarrow \mu \mu, Z / \gamma^{*} \rightarrow e e$, diboson samples ( $W W, Z Z$, and $W Z$ ), and the $t \bar{t}$ sample. If the $W+$ jet normalization, described in Section 7.7 is being applied, the $W+$ jet samples must be subtracted after the normalization.

These methods are designed to determine the multijet contributions at final selection, although they are applicable to other samples with the necessary $N N_{\tau}$ and $|Q|$ requirements. Therefore the TTNN and SR regions must have the same selection criteria as in the final selection regions, hence the value used 
for the $N N_{\tau}^{\text {tight }}$ requirement must match the $N N_{\tau}$ requirements as given in Sections 8.5.1 and 9.6.4. The values to use for the loose requirement on the $N N_{\tau}$ were investigated in Ref. [109]. It was determined that applying any loose $N N_{\tau}$ requirement reduces the statistics to the point they become too low to make an accurate estimation of the instrumental background events. The events at low $N N_{\tau}$ values below 0.3 are dominated by instrumental background events and do not have an efficiency correction applied, so including these events in the loose sample (LLNN or LR) could introduce discrepancies in the shape compared to the tight samples (TTNN or SR). Within the statistics available for these analyses the effects of including these events have been determined to be negligible [109].

\subsection{Multivariate Analysis Techniques}

In order to set limits on the cross section of the process being searched for, both the information from the number of predicted signal and background events selected by the analysis, and on their relative shape is used. Therefore a "final discriminate" distribution is needed that shows large discrimination between signal and background events. This distribution can either be a kinematic variable, for example, or a new distribution that is constructed specifically for this task. To construct such a variable a method known as a multivariate analysis, MVA, method, is used. This method is designed to exploit the discriminating power of multiple variables by using them to construct one variable with a high discriminating power. Examples of MVA techniques include Neural Networks, as used for the tau lepton and jet discrimination, and Decision Trees which will be used to constructed a final discriminant and will be discussed in this section. 


\subsubsection{Decision Trees}

A decision tree works on a binary structure, where decisions with a straight yes no answer, are repeatedly applied to a sample and each decision further splits up the sample into subsamples. Once some predefined level has been reached this process stops and and the subsamples are classified as either signal-like or backgroundlike depending on their composition [65]. This is shown in Figure 7.13, where it can be seen that from a single starting point the Decision Tree branches out, into many "leaves".. Running a tree through such a set of decisions is referred to as "training" the tree and each point of splitting is referred to as a node.

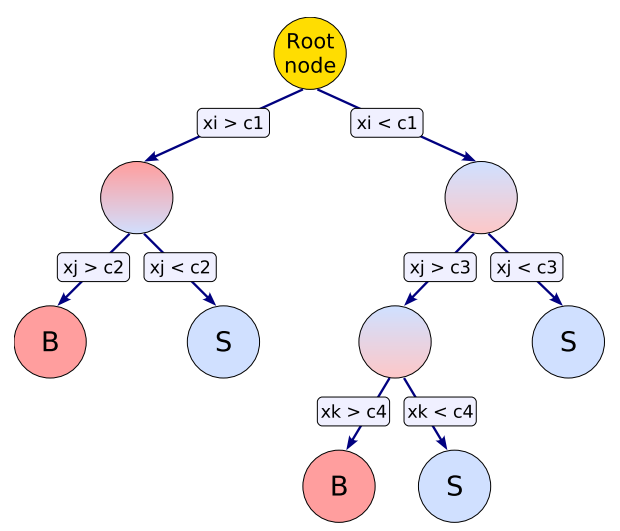

Figure 7.13: A diagrammatic representation of the structure of a decision tree [65], where each end node or leaf has been classified as either signal like, S, and background like, B.

\subsubsection{Boosted Decision Trees}

When producing the final discriminate an improved Decision Tree method known as a Boosted Decision Tree, BDT, is used. This is designed to both enhance the performance over a single Decision Tree and to reduce issues from statistical fluctuations arising from low statistics MC samples [65]. The idea is that events that are misclassified when a decision tree is first trained are reweighed with 
a higher event weight and then the tree is trained again, with these modified weights. This is repeated many times and the weighted average of the individual decision trees [65] is used as the output. This improves the performance by allowing for misclassified events to be re-trained and classified correctly hence improving stability against statistical fluctuations. There are various ways to achieve this "boosting".

\section{- Adaptive boost}

The most common type of boosting algorithm is an adaptive boost. This works by reweighting events by a boost weight, $\alpha$. The weight is determined from the error in classification of a sample, as either signal-like or background-like, err, [65],

$$
\alpha=\frac{1-e r r}{e r r}
$$

Defining a set of individual decision trees as $\mathrm{h}(\mathbf{x})$, where $\mathbf{x}$ is the vector of the input variables, which is encoded such that the signal is $\mathrm{h}(\mathbf{x})=+1$ and background is $\mathrm{h}(\mathbf{x})=-1[65]$. Then the BDT $y_{\text {Boost }}(\mathbf{x})$ can be written as

$$
y_{\text {Boost }}(\mathbf{x})=\frac{1}{N_{\text {collection }}} \cdot \sum_{i}^{N_{\text {collection }}} \ln \left(\alpha_{i}\right) \cdot h_{i}(\mathbf{x}) \text {, }
$$

where $N_{\text {collection }}$ is the number of events trained and $i$ is the number of Decision Trees. Large values of $y_{\text {Boost }}$ are defined to be signal-like and small values to be background-like.

\section{- Gradient Boost}

The adaptive boost method suffers from a couple of issues, most noticeably that it does not perform well where there are outlaying events or mislabeled data points [65]. A method called Gradient Boost can be used to overcome these issues, by allowing other forms of the boosting algorithm [65]. 


\section{- Bagging}

Bagging is not explicitly a boosting technique, thought it is often classified as such because it does not aim to enhance the Decision Tree method only to smear over statistical fluctuations in the training sample, therefore helping if the training sample is statistically limited [65]. The Bagging technique works on a Decision Tree being repeatably retrained on the same sample. The output is then determined from the average of these sets, which will reduce the sensitivity to statistical fluctuations.

\subsubsection{MVA procedure}

The MVA method employed, uses the TMVA package [65]. The BDT is trained on two MC samples, one for signal events and one for background events. The samples are a combination of all signal and background samples at final selection with all MC corrections and normalizations applied. In training a selection of variables are specified that have a shape difference between signal and background events, which the BDT is trained on. During training the signal and background samples are split into two equal parts, one of which is for training and the other for testing. Therefore enabling determination of weather the BDT is training on real features of the distributions or on fluctuations due to low MC sample statistics. It also shows if the BDT has too few degrees of freedom compared to the parameters of the algorithm [65]. If this is the case then the BDT is "overtrained" and the output from the training and testing samples will differ. An example output of a TMVA BDT is shown in Figure 7.14, where the signal events can be seen to peak at high values and the background events at low values of the BDT. 


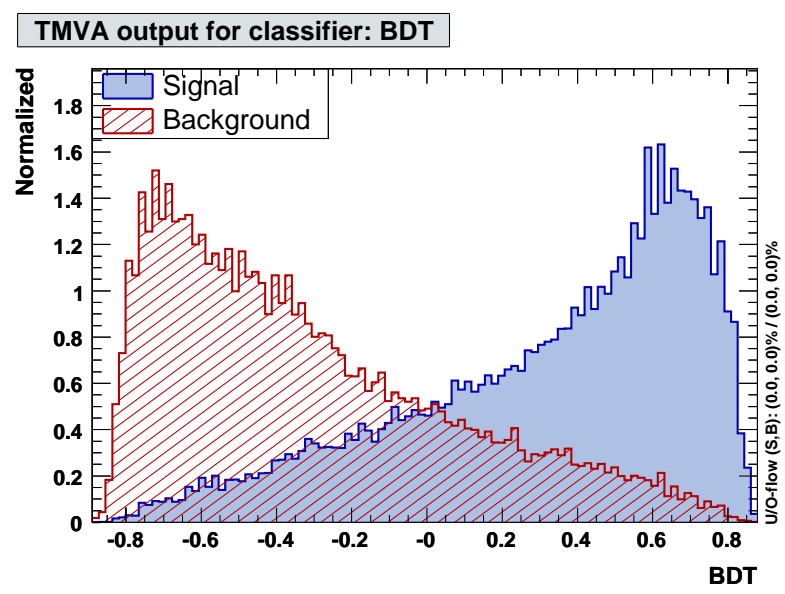

Figure 7.14: A example output of the BDT training, where the signal (blue) peaks at high values and the background (red) at low values [65].

TMVA provides tools to help distinguish the best input variables for the training, for example the correlation matrices for the training variables. These matrices consist of the linear correlation coefficients and are produced for both the signal and background training samples, enabling the determination which variables contain the same event information and to what extent.

The efficiency of the signal and background event rejection is also calculated by TMVA, and determines the optimal value at which the majority of the background events can be rejected while retaining the majority of the signal events. As a result TMVA can be used to create a high purity signal sample. An example efficiency distribution of the signal and background event rejection is shown in Figure 7.15 for the different MVA techniques that TMVA can calculate.

Once the BDT has been optimally trained it can be applied to the MC samples and observed data events at final selection. TMVA produces event weights as functions of the input training variables, which are applied to data and MC samples to produce a BDT output to be used as the final discriminant. The standard BDT training parameters as used by TMVA are listed in Table 7.9. 


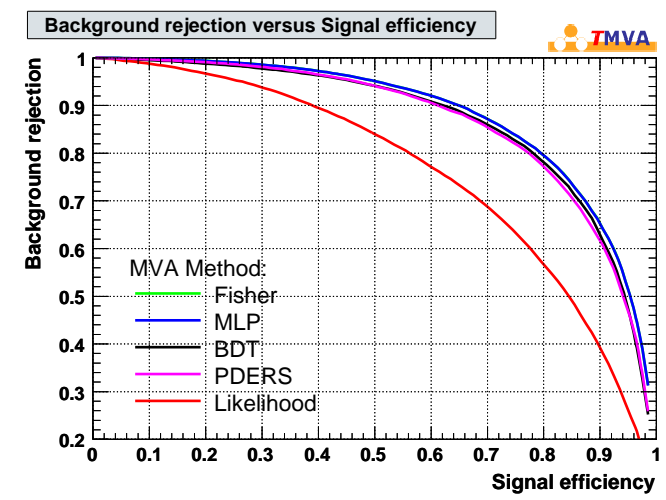

Figure 7.15: The example efficiency distribution for the signal and background rejection or all the different MVA techniques that TMVA can preform [65].

\begin{tabular}{|c|c|c|}
\hline Training Parameter & Description & Default \\
\hline NTrees & Number of trees in the forest & 200 \\
\hline Boost type & $\begin{array}{l}\text { Adaptive Boost, Gradient Boost or } \\
\text { Bagging (see Section 7.9.2) }\end{array}$ & Adaptive Boost \\
\hline UseRandomisedTrees & $\begin{array}{l}\text { At each splitting point a random } \\
\text { set of variables is chosen }\end{array}$ & False \\
\hline UseNvars & $\begin{array}{l}\text { Number of variables used } \\
\text { for the randomized tree option }\end{array}$ & 4 \\
\hline UseYesNoLeaf & $\begin{array}{l}\text { Use } S \text { and } B \text { categories or } \\
\text { purity as classification of the end node }\end{array}$ & True \\
\hline MaxDepth & Max depth of the decision tree allowed & 100000 \\
\hline NNodesMax & Max number of nodes in a tree & 100000 \\
\hline SeparationType & Separation criterion for node splitting & Gini Index $p \cdot(1-p)$ \\
\hline
\end{tabular}

Table 7.9: The standard TMVA training parameters, their description and default values. Purity is defined as $p=S /(S+B)$, where $S$ is the number of signal events at each node and $B$ is the number of background events. 


\subsection{Systematic Uncertainties}

There are two types of uncertainties that are necessary to account for, statistical and systematic. The statistical uncertainties are based on the number of data and $\mathrm{MC}$ events available and as the number of generated MC events is generally many orders of magnitude greater than the number of data events, the statistical uncertainty is generally only taken to be the statistical error from data, for which the analysis is limited by the experimental data recorded. The systematic uncertainties, are due to the method used. They include uncertainties on the object reconstruction and its efficiency, due to the trigger efficiency, on the luminosity measurement, and on the determined cross sections of the generated MC samples. The systematic uncertainties that are applicable to both analyses will be discussed here and analysis specific uncertainties in Sections 8.8 and 9.9.

\subsubsection{Cross section}

The theoretical cross sections of the background processes are known only to a limited accuracy due to both the calculation of the MEs and due to the PDF sets used [110]. This uncertainty therefore includes uncertainties on the k-factors used, on the scale uncertainty due to both the factorization and normalization scales, and the PDF uncertainties. The PDF uncertainties are determined by the CTEQ prescription, for which the cross sections are redetermined with a set of PDFs which represent the experimental error at the $1 \sigma$ level and the uncertainty is taken as the deviation from the central value [110]. The uncertainties are determined to be: $6 \%$ for $Z / \gamma^{*}, 10 \%$ for $t \bar{t}, 6 \%$ for $W+$ jet, and $7 \%$ for the diboson samples. 


\subsubsection{Luminosity}

The measured integrated luminosity has an associated uncertainty. This is determined from the Run IIa data and is due to the normalization used, the "back propagation" of correction functions used (the effect of extrapolating them back over a period of time), and the radiation damage to the luminosity monitor during Run IIa [111]. The uncertainty on the luminosity measurement was determined to be $6.1 \%$ [111].

\subsubsection{SingleMuonOR trigger}

The uncertainty on the efficiency of the SingleMuonOR trigger is determined by calculating the efficiency with the tag and probe method and with an independent trigger method [56]. The SingleMuonOR trigger efficiency has been determined to be $5 \%$.

\subsubsection{Muon Quality}

The uncertainty on the efficiencies for muon identification are determined separately for the track reconstruction, muon isolation, and the muon identification quality requirement. As the quality definitions used vary for the analyses performed the sources of uncertainties will be described here but the values specific to each given analysis will be given in their respective chapters, in Sections 8.8 and 9.9 .

\section{Muon identification efficiency}

This uncertainty on the muon identification efficiency is due to bias from the tag and probe method used, the uncertainties due to the sample used for the study, and the differences seen due to the variation in the $p_{T}$ of the $Z$ boson [101]. 
For muons with a $p_{T}<20 \mathrm{GeV}$ an additional fractional $2 \%$ uncertainty must be included to account for additional tag and probe biases in this low $p_{T}$ region [101].

\section{Muon track efficiency}

For the track, the efficiency uncertainty is strongly dependent on the hadronic activity of the event and hence is strongly correlated with the $p_{T}$ of the $Z$ boson [101]. The modeling of this shows differences between data and MC simulations and an uncertainty is included to account for this. As the hadronic activity will vary for different final states, the error depends on the final states studied. As the efficiency is parametrized in $\eta$, if the $\eta$ distribution differs between the analysis sample and the sample used to determine the correction, an additional uncertainty can be induced. Additional sources of uncertainties are the tag and probe method and the sample used to perform the study.

\section{Muon isolation efficiency}

The uncertainty for the muon isolation efficiency is dependent on the same factors as the uncertainty for the muon identification efficiency. These are the tag and probe method, the sample used to perform the study and tag and probe biases in the low $p_{T}$ region [101].

\subsubsection{Tau identification}

\section{$\mathrm{NN}_{\tau}$ efficiency}

The $N N_{\tau}$ efficiency correction is explained in detail Section 7.4.6, and is determined separately the two $N N_{\tau} \mathrm{s}$ used, $N N_{2010}$ or $N N_{2012}$. For both $N N_{\tau}$ s the uncertainty is dependent on the selection requirement placed on the $N N_{\tau}$, along with the multijet method used in determining the efficiency, and the kinematics of the tau lepton candidates. To estimate these contributions two separate multijet 
estimations are used with the difference between then taken as the uncertainty and the correction is derived both for when the $p_{T}$ of the tau lepton is between 15-25 GeV and when it is greater than $25 \mathrm{GeV}$, with the difference from the nominal value being taken as the uncertainty. The uncertainties per tau lepton type, as a function of the $N N_{\tau}$ are shown in Figure 7.5 (bottom) for the $N N_{2010}$ and in Figure 7.6 (bottom) for the $N N_{2012}$. As both the $N N_{\tau}$ used and the associated requirement vary between the two analyses preformed the specific values will be given in the Sections 8.8 and 9.9.

\section{Tau lepton track efficiency}

The efficiency for the tau lepton track correction is taken from the studies performed for the muon track efficiency as described in Section 7.4.4. The uncertainty is hence taken from the same studies and is determined as described for the muon. It has been determined to be $1.4 \%$ [101].

\section{Tau lepton energy scale}

The uncertainty on the tau lepton energy scale affects both the shape and the efficiency. The uncertainty is determined from the deviation seen in the ratio $E_{c a l}^{T} / p_{T}$ (track) after the tau lepton energy scale is applied. The ratio is seen to show only very small deviations at low values less than $20 \mathrm{GeV}[112,109]$. Therefore a systematic of $1 \%$ is applied.

\subsection{Cross section limits}

In order to perform a search using the final discriminant one final step is needed. A method by which to perform statistical analysis on that distribution, to determine the probability if the signal process searched for is contained within that data sample. If by this method a statistical excess of data consistent with the signal 
process searched for is seen, then evidence can be claimed for seeing that signal. When no excess is seen, then either limits can be set on the cross section of the signal process, for the case when the search does not have the sensitivity to exclude that signal process, or if this sensitivity does exists, exclude the signal process. As cross sections are mass dependent and the mass of the signal is not generally known, then limits are set across the mass range that coincides both with the range the particle's mass is expected to reside in and the range accessible with the experimental apparatus used. At D $\varnothing$ the program used to perform these statistical calculations is COLLIE [113].

The final discriminant is designed to provide maximum separation between two distinct hypothesis, the NULL hypothesis, that there is no contribution from the signal process, also known as the background only hypothesis, B, and the TEST hypothesis, that there is a contribution from the signal process, know as the signal plus background hypothesis, $\mathrm{S}+\mathrm{B}$. Collie is designed to test both of these hypotheses against the observed data. These hypotheses can be written as,

$$
\begin{aligned}
& \text { NULL : } N_{\text {events }}^{\mathrm{NULL}}\left(\phi_{B}\right)=L \times \epsilon_{B}\left(\phi_{B}\right) \times \sigma_{B} \\
& \operatorname{TEST}: N_{\text {events }}^{\mathrm{TEST}}\left(\phi_{S}, \phi_{B}\right)=L \times \epsilon_{B}\left(\phi_{B}\right) \times \sigma_{B}+L \times \epsilon_{S}\left(\phi_{S}\right) \times \sigma_{S}
\end{aligned}
$$

where $L$ is the total integrated luminosity of the data, $\epsilon_{B}$ and $\epsilon_{S}$ are the efficiencies of the signal and background respectively, $\sigma_{B}$ and $\sigma_{S}$ are the cross sections of the background and signal samples, and $\phi_{B}$ and $\phi_{S}$ represent the uncertainties on the number of signal and background events. Therefore the TEST hypothesis is simply the NULL hypothesis in the case that the signal process cross section is not zero, i.e. the TEST hypothesis contains a additional term as compared to the NULL hypothesis, that consists of the parameter of interest (the signal cross section), along with other parameters that can affect the result but are not what is being measured ( $L$ and $\epsilon_{S}$ ), so called nuisance parameters.

A statistical test $\Gamma(\mathrm{N})$ can be performed on these two hypotheses to determine 
which one the observed data most reflects, where $\mathrm{N}$ can be one of three cases,

1. $N=N_{\text {events }}^{\text {observed }}$ the number of data events observed,

2. $N=N_{\text {events }}^{T E S T}$, the number of events predicted by the TEST hypothesis, the total number of predicted signal and background events, $S+B$,

3. $N=N_{\text {events }}^{N U L L}$, the number of events predicted by the NULL hypothesis, the total number of predicted background events, $B$.

The outcome of repeated experiments must be modeled to determine the probability of the outcome being seen, i.e. probability distribution functions, pdfs, must be derived for both the NULL and TEST hypotheses. It is assumed that the observed data has a Poisson distribution and that the observed data is a random sampling taken from that distribution, where the mean value of that distribution is given by the expected number events for either the NULL or TEST hypothesis, $N_{\text {events }}^{\text {NULL }}$ or $N_{\text {events }}^{\text {TEST }}$. Therefore two pseudo-experiments can be performed, one for each hypotheses, producing two sets of pseudo-data derived from the Poisson distributions for the NULL and TEST hypotheses. These sets of pseudo-data will describe the pdfs of the two hypotheses.

This picture becomes more complicated when nuisance parameters are included, as these have a uncertainty associated with them, i.e. the value of the efficiency for the signal, $\epsilon_{S}$, has a defined uncertainty, $\phi_{S}$, so values of $N_{\text {events }}^{\text {TEST }}$ can vary by some corresponding amount. Therefore as these nuisance parameters vary over the allowed range as defined by their uncertainties, the nominal background prediction for each hypothesis will change, $\left(N_{\text {events }}^{\mathrm{NULL}}\right.$ or $\left.N_{\text {events }}^{\mathrm{TEST}}\right)$, and therefore the mean of the Poisson distribution for the two pesudo-experiements will change and hence so will the generated pseudo-data. From the predefined allowed values of the uncertainties on the nuisance parameters, a superposition of hypotheses can be created, each with different value of the nuisance paramerters 
that has been defined as possible, hence allowing all the possible outcomes for a hypothesis to be specified. This set of all possible outcomes for a hypothesis is commonly referred to as the "prior predictive ensemble" [113]. For every value in this ensemble a statistical test can now be performed providing the pdfs for the two hypotheses.

The statistical test needs to be designed to maximize both the signal density and the isolation of signal from background events [113] and is defined in terms of a statistical significance that it is required to achieve. Defining a variable $x$ that the outcome depends on, a statistical test can be classified as regions, containing $x$, where the pdf ratios of the two hypotheses achieve the wanted statistical significance. This can be written [113],

$$
Q(x)=\frac{f(x \mid T E S T)}{f(x \mid N U L L)}
$$

For the statistical test COLLIE, uses a Poisson likelihood-ratio, which assumes that the two hypotheses are treated as counting experiments, with numbers of signal, $s$, background, $b$, and data, $d$, events, can be written as [113],

$$
Q(s, b, d)=\frac{\left(s_{b}\right)^{d} e^{-(s+b)} / d !}{b^{d} e^{-b} / d !}
$$

where $s$ and $b$ are the values given for the signal and background from MC simulations and $d$ is the number of data events either observed or generated as pseudo data for one of the two test hypothesis

In order for COLLIE to take into account the shape distribution of the final discriminant distributions, and not simply the numbers of events as been discussed so far, COLLIE requires that the input distributions for the observed data, signal, and background events be binned. It then performs the pseudo-experiments, including uncertainties from the nuisance parameters, in each bin. COLLIE also 
allows for several different final state channels to be combined. Therefore Equation 7.14 can be expanded to [113],

$$
\begin{aligned}
Q(s, b, d) & =\prod_{i=0}^{N_{\text {Channels }}} \prod_{j=0}^{N_{\text {bins }}}\left[\frac{\left(s_{b}\right)_{i j}^{d_{i j}} e^{-(s+b)_{i j}}}{d_{i j} !}\right] /\left[\frac{b^{d_{i j}} e^{-b_{i j}}}{d_{i j} !}\right] \\
& =\prod_{i=0}^{N_{\text {Channels }}} \prod_{j=0}^{N_{\text {bins }}} e^{-\left(s_{i j}\right)}\left(\frac{s_{i j}+b_{i j}}{b_{i j}}\right)^{d_{i j}}
\end{aligned}
$$

where $i$ is the number of channels and $j$ the number of bins. A mathematically compact form can be achieved by using the negative-log-likeihood ratio, NLLR, [113], or LLR as the negative is often dropped, as shown in Equation 7.16.

$$
\operatorname{LLR}(s, b, d)=-2 \log (Q)=\sum_{i=0}^{N_{\text {Channels }}} \sum_{j=0}^{N_{\text {bins }}} s_{i j}-d_{i j} \ln \left(1+\frac{s_{i j}}{b_{i j}}\right)
$$

This is the form of the statistical test as used by COLLIE and in shown in Figure 7.16 as a function of the probability density. In this figure it can be seen that the observed LLR is equal to the NULL hypothesis.

In general the observed LLR will not match the NULL hypothesis and an example is shown in Figure 7.17, where the LLR distribution is plotted as a function of a parameter that the signal cross section is dependent on. This parameter would be the Higgs boson mass in the searches performed in this thesis. The separation of the $\mathrm{LLR}_{B}$ and $\mathrm{LLR}_{S+B}$ gives an indication of the discriminating power of the search performed and the separation of these values from the $\mathrm{LLR}_{\text {obs }}$ gives an indication how signal-like or background-like the observed data is. The width of the $1 \sigma$ and $2 \sigma$ bands gives a measure how sensitive an analysis is to seeing the predicted signal process in the observed data.

As shown in Equation 7.13 the statistical test is defined as requiring regions where the ratio of the two pdfs achieve a certain statistical significance [113]. This significance is defined through "Confidence Levels", C.L.. These levels are 


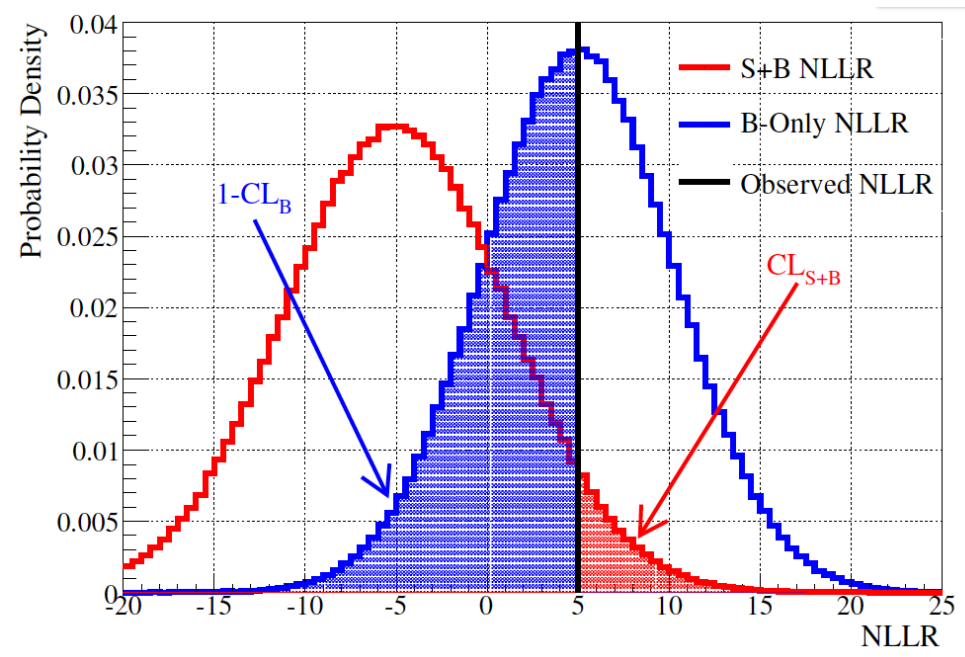

Figure 7.16: The probability density of the LLR distribution, showing the probability distribution for NULL (background only) hypothesis as the blue curve and the TEST (background plus signal) hypothesis as the red curve. The observed LLR is shown in black and the shaded regions correspond to $C L_{S+B}$ (red) and $1-C L_{B}$ (blue) [113].

constructed based on the outcomes of the statistical test for a specific hypothesis, with each outcome ordered according to the determined LLR value and the frequency of a specific outcome defining the confidence levels [113]. Two confidence levels are defined, $C L_{S+B}$, the probability for the TEST hypothesis to be more background-like than is observed in the data and $C L_{B}$, the probability for the NULL hypothesis to produce an outcome more background-like than that observed in the data. To determine these levels the pdfs are integrated from the observed outcome, as can be seen in Figure 7.16, where $C L_{S+B}$ is shown as shaded red region and $1-C L_{B}$ as the region shaded in blue.

These regions can be used to then determine limits on the parameter studied (the predicted Higgs boson cross section for the analysis performed here). It is possible to set limits using only $C L_{S+B}$ but this can produce false exclusions when 


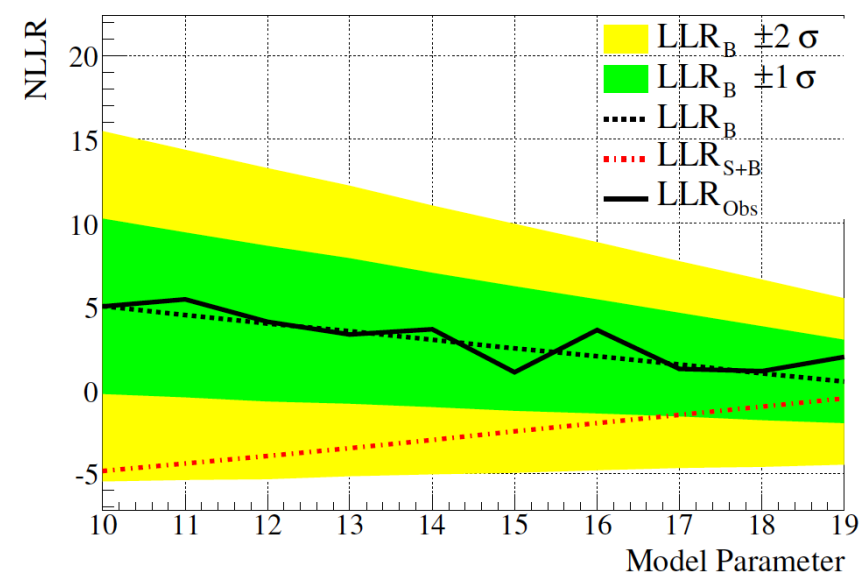

Figure 7.17: The LLR distribution as a function of a model parameter, which predicted signal is dependent on. The determined $\operatorname{LLR}_{B}$ is showed in dotted black, the $\operatorname{LLR}_{S+B}$ in dotted red, the observed LLR in solid black, the one and two $\sigma$ deviations from the determined $\mathrm{LLR}_{B}$ are shown green and yellow respectively [113].

there are downward fluctuations of the data [113]. To avoid this the modifedFrequentist statistic, $C L_{S}$, is used,

$$
C L_{S}=\frac{C L_{S+B}}{C L_{B}}
$$

This value will have a dependence on the expected signal as a function of the parameter studied (in this case the cross section of the Higgs boson), i.e. $C L_{S}=$ $C L_{S}(s(x))$, where $s$ is the signal rate and $x$ the model parameter. Therefore the limits determined will be dependent on these variables. The condition for exclusion is defined such that $C L_{S}(s(x))<\alpha$, where $\alpha$ is a fractionial C.L. and excludes the signal at $1-\alpha$. For the searches performed in this thesis $\alpha=0.05 \%$ and hence exclusions are performed at the $95 \%$ C.L.. 


\section{Chapter 8}

\section{Doubly charged Higgs boson pair production}

In this chapter full details of a search for the pair production of doubly charged Higgs bosons in the process $q \bar{q} \rightarrow H^{++} H^{--}$, decaying to $\tau \tau, \mu \mu$, or $\tau \mu$ lepton pairs, using a total integrated luminosity of $L=7.0 \mathrm{fb}^{-1}$, are given, as published in Ref. [114].

As covered in Section 2.7.2, doubly charged Higgs, $H^{ \pm \pm}$, bosons, could be pair produced at the Tevatron, $p \bar{p} \rightarrow Z / \gamma^{*} \rightarrow H^{++} H^{--}$, or be produced singly with a charged Higgs boson, $p \bar{p} \rightarrow W^{ \pm} \rightarrow H^{ \pm \pm} H^{\mp}$. As the single production mode of $H^{ \pm \pm}$Higgs bosons is more model dependent, it will be neglected. $H^{ \pm \pm}$bosons could decay to pairs of same electric charge leptons, $q \bar{q} \rightarrow H^{++} H^{--} \rightarrow \ell^{+} \ell^{+} \ell^{-} \ell^{-}$, as shown in Figure 8.1. Experimental limits on the coupling of $H^{ \pm \pm}$bosons to muons and electrons exist, with the most stringent limits placed on the coupling of a $H^{ \pm \pm}$boson to electrons. Therefore this decay channel of $H^{ \pm \pm}$boson to electrons will not be considered. There are no limits on the coupling of $H^{ \pm \pm}$ bosons to tau leptons [11]. Therefore decays into tau leptons and muons will be considered. All leptonic $H^{ \pm \pm}$boson decays are lepton number violating therefore decays to off diagonal states, $\tau \mu$ lepton pairs, will also be considered. 


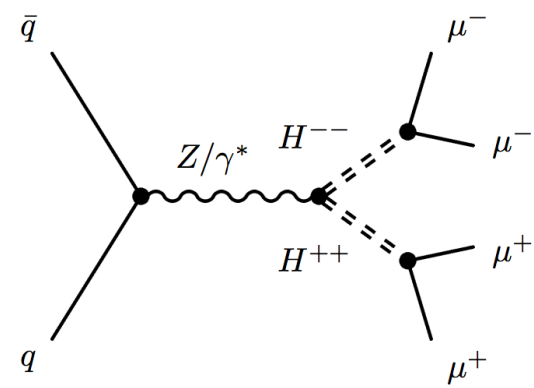

Figure 8.1: A Feynman diagram showing the pair production of a $\mathrm{H}^{++} \mathrm{H}^{--}$boson pair with a $100 \%$ branching ratio into muons [44].

Five different benchmark points will be studied four of which are not specific to a $H^{ \pm \pm}$model and one that is motivated by Little Higgs models, which predict for the normal hierarchy of neutrino masses that $H^{ \pm \pm}$bosons will decay with equal branching ratios, $\mathcal{B} s$, to $\mu \mu, \tau \tau$, and $\mu \tau$ lepton pairs [22]. For the four non model specific modes both left and right handed $H^{ \pm \pm}$bosons will be considered for the results to be applicable to Left-Right symmetric models. The handedness refers to that of their decay products, as the $H^{ \pm \pm}$carries no spin. Any $\nu_{R}$ introduced in these models is assumed to be much heavier than the $H^{ \pm \pm}$boson and not take part in their decays. Therefore kinematic differences are expected depending on electric charge and handedness of the tau leptons.

1. $H^{ \pm \pm}$decaying to tau leptons $\mathcal{B}\left(H^{ \pm \pm} \rightarrow \tau \tau\right)=1,\left(\mathrm{~B}_{\tau \tau}=1\right)$.

2. Mixed branching ratio to tau leptons and muons, $\mathcal{B}\left(H^{ \pm \pm} \rightarrow \tau \tau\right)+\mathcal{B}\left(H^{ \pm \pm} \rightarrow\right.$ $\mu \mu)=1,\left(\mathrm{~B}_{\tau \tau}+\mathrm{B}_{\mu \mu}=1\right)$. The contributions from the two decays are ranged from, $\mathcal{B}\left(H^{ \pm} \rightarrow \tau \tau\right)=90 \%, \mathcal{B}\left(H^{ \pm} \rightarrow \mu \mu\right)=10 \%$, to $\mathcal{B}\left(H^{ \pm} \rightarrow \tau \tau\right)=10 \%$, $\mathcal{B}\left(H^{ \pm} \rightarrow \mu \mu\right)=90 \%$.

3. $H^{ \pm \pm}$decaying to muons $\mathcal{B}\left(H^{ \pm \pm} \rightarrow \mu \mu\right)=1,\left(\mathrm{~B}_{\mu \mu}=1\right)$.

4. $H^{ \pm \pm}$decaying to tau lepton/muon pairs $\mathcal{B}\left(H^{ \pm \pm} \rightarrow \mu \tau\right)=1,\left(\mathrm{~B}_{\mu \tau}=1\right)$. 
5. The Little Higgs model specific $H^{ \pm \pm}$boson decay mode, for which only left handed $H^{ \pm \pm}$bosons are considered. $H^{++} H^{--}$pair decaying with equal branching ratio to $\tau \tau, \mu \mu$, and $\tau \mu$ pairs, $\mathcal{B}\left(H^{ \pm \pm} \rightarrow \tau \tau\right)=\mathcal{B}\left(H^{ \pm \pm} \rightarrow \mu \mu\right)$ $=\mathcal{B}\left(H^{ \pm \pm} \rightarrow \mu \tau\right)=\frac{1}{3},\left(\mathrm{~B}_{\tau \tau}+B_{\tau \tau}+\mathrm{B}_{\tau \tau}=1 / 3\right)$.

\subsection{Data samples}

The analysis presented here uses the full Run IIb $1-3$ data and the first $0.7 \mathrm{fb}^{-1}$ of integrated luminosity of the Run IIb 4 data samples, using physics Runs taken within the range 221698 to 262856 . This corresponds to a total integrated luminosity of $L=7.0 \mathrm{fb}^{-1}$. This analysis uses the MaTau skims (see Section 7.1) for Run IIb 1, 2, and 3 data. As only part of the Run IIb 4 data was used, this data portion was processed directly from the MUinclusive skim, with the same quality requirements, as for the Run IIb 1 - 3 MuTau skims. The events used in this analysis are required to pass the SingleMuonOR trigger, described in Section 4.1, and the data quality requirements as covered in Section 7.1.2.

\subsection{Monte Carlo samples}

The generated MC samples for both $H^{ \pm \pm}$boson signal production and decays modes studied, and the SM background processes, will be summarized in this Section.

\subsubsection{Background Monte Carlo samples}

The background MC samples described in Section 7.3 and listed in Tables 7.3 and 7.4 are used. These are reweighted to the analyzed integrated luminosity of $7.0 \mathrm{fb}^{-1}$ and have the SingleMuonOR trigger efficiencies applied. The k-factors 
given in Table 7.5 are applied, along with the correction factors as described in Section 7.4.

\subsubsection{Signal Monte Carlo samples}

Signal samples for the pair production of the doubly charged Higgs boson are generated using the PYTHIA event generator, version 6.323 [62], with the tau lepton decays processed through TAUOLA [78]. Samples were generated for both the left handed and right handed doubly charged Higgs bosons, with the PYTHIA processes denoted by $\operatorname{MSUB}(349)=1$ and $\operatorname{MSUB}(350)=1$, respectively.

Samples are produced for two exclusive decay modes, for benchmark points (1) and (4), where the $H^{ \pm \pm}$boson has $\mathcal{B}\left(H^{ \pm \pm} \rightarrow \tau \tau\right)=1$ and $\mathcal{B}\left(H^{ \pm \pm} \rightarrow \tau \mu\right)=$ 1. One mixed decay, where the $H^{ \pm \pm}$decays to both tau leptons and muons, $H^{ \pm \pm} H^{\mp \mp} \rightarrow \tau^{ \pm} \tau^{ \pm} \mu^{\mp} \mu^{\mp}$, is also produced. This sample can be combined with other samples, to study benchmark point (2). For the left handed $H^{ \pm \pm}$boson, a sample was also produced for benchmark point (5), for a decay with equal branching ratios to $\tau \tau, \tau \mu$, and $\mu \mu$ lepton pairs, $\mathcal{B}\left(H^{ \pm} \rightarrow \tau \tau\right)=\mathcal{B}\left(H^{ \pm} \rightarrow \mu \tau\right)=$ $\mathcal{B}\left(H^{ \pm} \rightarrow \mu \mu\right)=1 / 3$. Contributions from $H^{ \pm \pm} H^{ \pm \pm} \rightarrow \tau \tau \tau \tau, \tau \tau \mu \mu, \tau \tau \mu \tau, \mu \mu \mu \mu$, $\mu \mu \mu \tau$ and $\mu \tau \mu \tau$ are all included in this sample. The signal samples generated are summarized in Table 8.1 for both the left handed and the right handed states. The range of masses that the samples are generated for is also shown.

\subsection{Signal cross section normalization}

The signal cross section for the process $q \bar{q} \rightarrow Z / \gamma^{*} \rightarrow H^{++} H^{--}$depends only on the mass of the $H^{ \pm \pm}$boson. The cross section for both left handed and right handed $H^{ \pm \pm}$bosons, normalized to NLO cross sections, as calculated by [115], is given in Table 8.2. The NLO corrections have been shown to increase the 


\begin{tabular}{c|r|c} 
Masses of $H^{ \pm \pm}$bosons generated $[\mathrm{GeV}]$ & Decay & Sample Name \\
\hline Left Handed Samples & & \\
\hline $100,120,140,160,180,200$ & $\mathcal{B}\left(H^{ \pm \pm} \rightarrow \tau \tau\right)=1$ & $4 \tau$ \\
$100,120,140,160,180,200$ & $H^{ \pm \pm} H^{\mp \mp} \rightarrow \mu \mu \tau \tau$ & $2 \tau 2 \mu$ \\
$100,120,140,160,180,200$ & $\mathcal{B}\left(H^{ \pm \pm} \rightarrow \mu \tau\right)=1$ & $\mu \tau$ \\
$100,120,140,160,180,200$ & Equal $\mathcal{B}$ & Equal $\mathcal{B}$ \\
\hline Right Handed Samples & & \\
\hline $90,100,120,140,160,180,200$ & $\mathcal{B}\left(H^{ \pm \pm} \rightarrow \tau \tau\right)=1$ & $4 \tau$ \\
$90,100,120,140,160,180,200$ & $H^{ \pm \pm} H^{\mp \mp} \rightarrow \mu \mu \tau \tau$ & $2 \tau 2 \mu$ \\
$90,100,120,140,160,180,200$ & $\mathcal{B}\left(H^{ \pm \pm} \rightarrow \mu \tau\right)=1$ & $\mu \tau$
\end{tabular}

Table 8.1: The signal samples generated for the $H^{ \pm \pm}$boson analysis for both left and right handed $H^{ \pm \pm}$bosons, where "equal $\mathcal{B}$ " is $\mathcal{B}\left(H^{ \pm \pm} \rightarrow \tau \tau\right)=\mathcal{B}\left(H^{ \pm \pm} \rightarrow\right.$ $\mu \mu)=\mathcal{B}\left(H^{ \pm \pm} \rightarrow \mu \tau\right)=\frac{1}{3}$.

cross section by $20-30 \%$. The NNLO corrections, which have been shown to be of order $5-10 \%$ [116], are not included. The uncertainty on the renormalization and factorisation scale at NLO $(5-10 \%)$ is used as an estimate of the theoretical uncertainties. These are combined with the uncertainties on the parton densities to give a total uncertainty of $10-15 \%[115,117]$. A value of $10 \%$ is taken as the uncertainty on the theoretical cross section. The right handed $H^{ \pm \pm}$boson is predicted in left-right symmetric models to have a weaker coupling to the $Z$ boson, reflected in the approximate factor of two reduction in the cross section.

\subsection{Selection requirements}

\subsubsection{Pre-selection requirements}

In addition to the tau lepton and muon pre-selection requirements, as described in Section 7.5, the following requirements are applied:

- The muon is required it to be of "Medium" muon quality, "TrackMedium" track quality and "NPTight" isolation requirements, defined in Section 4.3.1. 


\begin{tabular}{c|c|c|c|c}
$M\left(H^{ \pm \pm}\right)$ & \multicolumn{2}{|c|}{ Left handed } & \multicolumn{2}{c}{ Right handed } \\
{$[\mathrm{GeV}]$} & $\sigma_{\mathrm{LO}}[\mathrm{fb}]$ & $\sigma_{\mathrm{NLO}}[\mathrm{fb}]$ & $\sigma_{\mathrm{LO}}[\mathrm{fb}]$ & $\sigma_{\mathrm{NLO}}[\mathrm{fb}]$ \\
\hline 80 & 216.5 & 291.2 & 93.4 & 125.4 \\
90 & 136.9 & 183.8 & 60.1 & 80.6 \\
100 & 90.1 & 120.7 & 40.1 & 53.6 \\
110 & 61.1 & 81.6 & 27.5 & 36.6 \\
120 & 42.4 & 56.4 & 19.2 & 25.6 \\
130 & 30.0 & 39.7 & 13.7 & 18.1 \\
140 & 21.4 & 28.3 & 9.86 & 13.0 \\
150 & 15.5 & 20.5 & 7.19 & 9.47 \\
160 & 11.4 & 14.9 & 5.29 & 6.94 \\
170 & 8.39 & 11.0 & 3.92 & 5.12 \\
180 & 6.24 & 8.14 & 2.92 & 3.81 \\
190 & 4.66 & 6.06 & 2.19 & 2.85 \\
200 & 3.50 & 4.54 & 1.65 & 2.14
\end{tabular}

Table 8.2: LO and NLO cross sections for the pair production of left handed and right handed $H^{ \pm \pm}$bosons.

- Only Type-1 and Type-2 two tau lepton candidates are considered in this analysis. The inclusion of Type-3 tau lepton candidates was seen to increase the background significantly without any significant gain in predicted signal events.

- $\Delta z\left(\tau_{i}, \mu\right)<2 \mathrm{~cm}$ where $i=1,2$. The distance between the vertices of the tau leptons and the muon is required to be less than $2 \mathrm{~cm}$ in the $z$ plane. This ensures that all three particles originate from a common vertex.

- All three lepton pairs are required to be isolated, $\Delta R\left(\ell_{i}, \ell_{j}\right)>0.5$, where $\ell=\tau_{1}, \tau_{2}$ or $\mu$.

\subsubsection{Distributions at pre-selection}

The kinematic distributions of the data events compared to the predicted MC events at pre-selection, with all MC event corrections and normalizations applied (Section 7.4), are shown in Figures 8.2 and 8.3. Samples that have only a small 
contribution at final selection are shown together. These are $W+$ jet, $Z / \gamma^{*} \rightarrow e e$, and $t \bar{t}$ pairs. Table 8.4 lists the event yields of the observed data and predicted MC backgrounds at this selection.

The multijet event contribution at this selection can be considered to be purely illustrative. The multijet estimation methods described in Section 7.8.1 and Section 7.8.2, are designed to be used with a $N N_{\tau}$ requirement, which is not applied until final selection. Adapting these methods to the pre-selection sample leads to a large correlation between the sample used for determining the shape of the multijet events and the sample for which the estimation is being applied to.

The signal distributions for a left handed $H^{ \pm \pm}$boson with $M_{H^{ \pm \pm}}=120 \mathrm{GeV}$ are shown superimposed on these distributions, enhanced by a factor of 50 . Three cases are shown, $\mathcal{B}\left(H^{ \pm \pm} \rightarrow \tau \tau\right)=1, \mathcal{B}\left(H^{ \pm \pm} \rightarrow \mu \tau\right)=1$ and the equal $\mathcal{B}$ case where $\mathcal{B}\left(H^{ \pm \pm} \rightarrow \tau \tau\right)=\mathcal{B}\left(H^{ \pm \pm} \rightarrow \mu \mu\right)=\mathcal{B}\left(H^{ \pm \pm} \rightarrow \mu \tau\right)=\frac{1}{3}$. The following distributions are shown:

- Transverse momenta of the two tau leptons and the muon, $p_{T}\left(\tau_{1}\right), p_{T}\left(\tau_{2}\right)$, and $p_{T}(\mu)$.

- $\Delta R$ between the three lepton pairs: $\Delta R\left(\tau_{1}, \tau_{2}\right), \Delta R\left(\tau_{1}, \mu\right)$, and $\Delta R\left(\tau_{2}, \mu\right)$.

- Number of muons, $N_{\mu}$, number of tau leptons, $N_{\tau}$, and the transverse mass, $M_{T}=\sqrt{2 P^{\mu} E^{\nu}\left(1-\cos \left(\Delta \phi_{\mu \nu}\right)\right)}$, where $\Delta \phi_{\mu \nu}$ is the angle between the muon and the $\mathbb{F}_{\mathrm{T}}$ in the $r \phi$ plane.

- Missing transverse energy, $\mathbb{E}_{\mathrm{T}}$, the invariant mass of the two highest $p_{T}$ tau leptons, $M\left(\tau_{1}, \tau_{2}\right)$, and the invariant mass of the two highest $p_{T}$ tau leptons and the highest $p_{T}$ muon, $M\left(\mu, \tau_{1}, \tau_{2}\right)$.

- The $\eta$ and $\phi$ angular distributions for the two highest $p_{T}$ tau leptons and the muon, $\eta(\mu), \eta\left(\tau_{i}\right), \phi(\mu)$, and $\phi\left(\tau_{i}\right)$. 

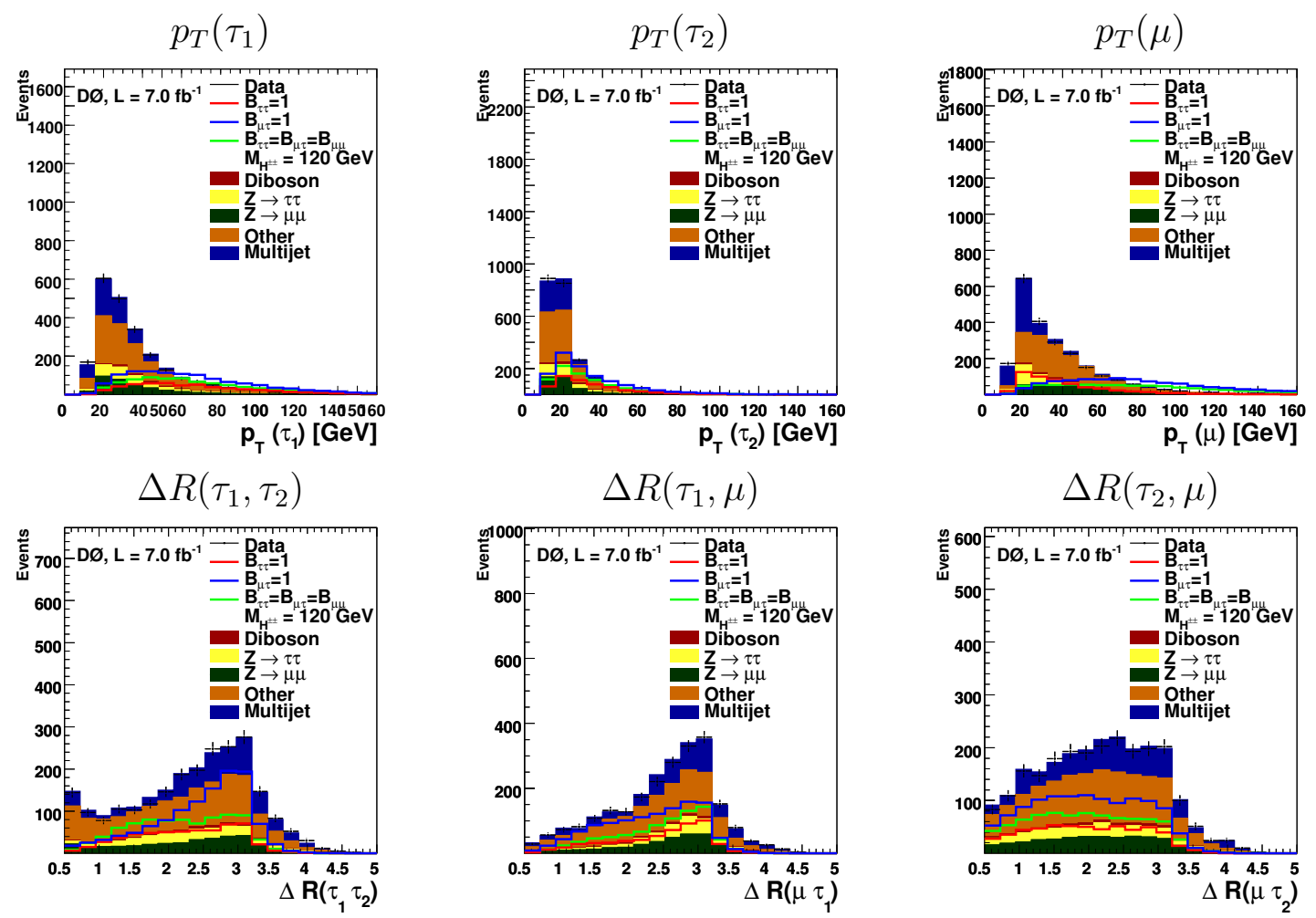

$N_{\mu}$

$N_{\tau}$
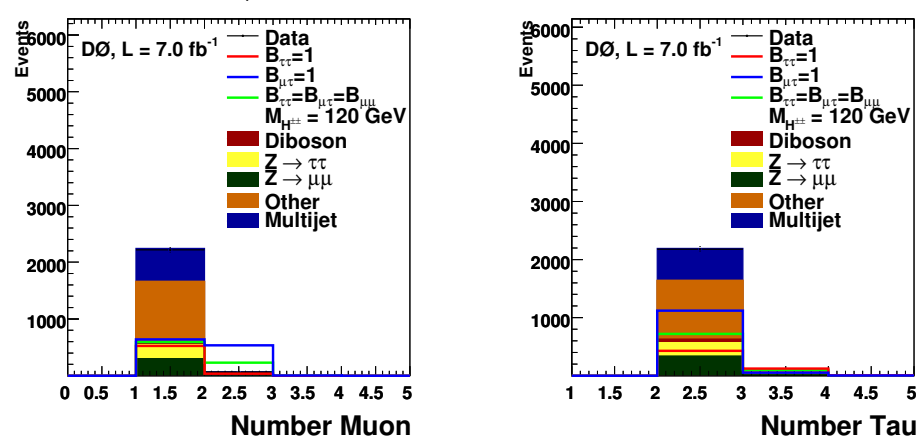

$M_{T}$

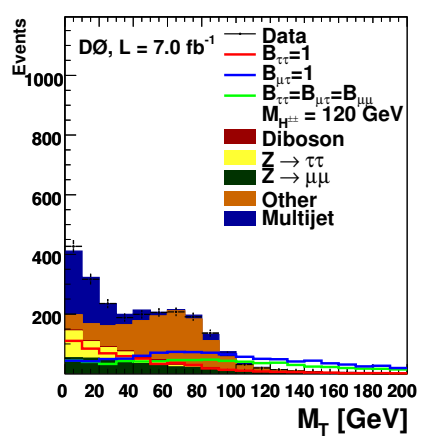

Figure 8.2: Data distributions at pre-selection compared to the sum of the expected backgrounds from MC simulations and multijet estimations. The signal distributions at pre-selection for a $M_{H^{ \pm \pm}}=120 \mathrm{GeV}$ left handed pair produced $H^{ \pm \pm}$boson for the cases where $B_{\tau \tau}=1, B_{\mu \tau}=1$, and $B_{\tau \tau}=B_{\mu \mu}=B_{\mu \tau}=\frac{1}{3}$. multiplied by a factor of 50, are superimposed. The 'Other' background sample contains the contributions from $Z \rightarrow e e, W+$ jets and $t \bar{t}$ production. 

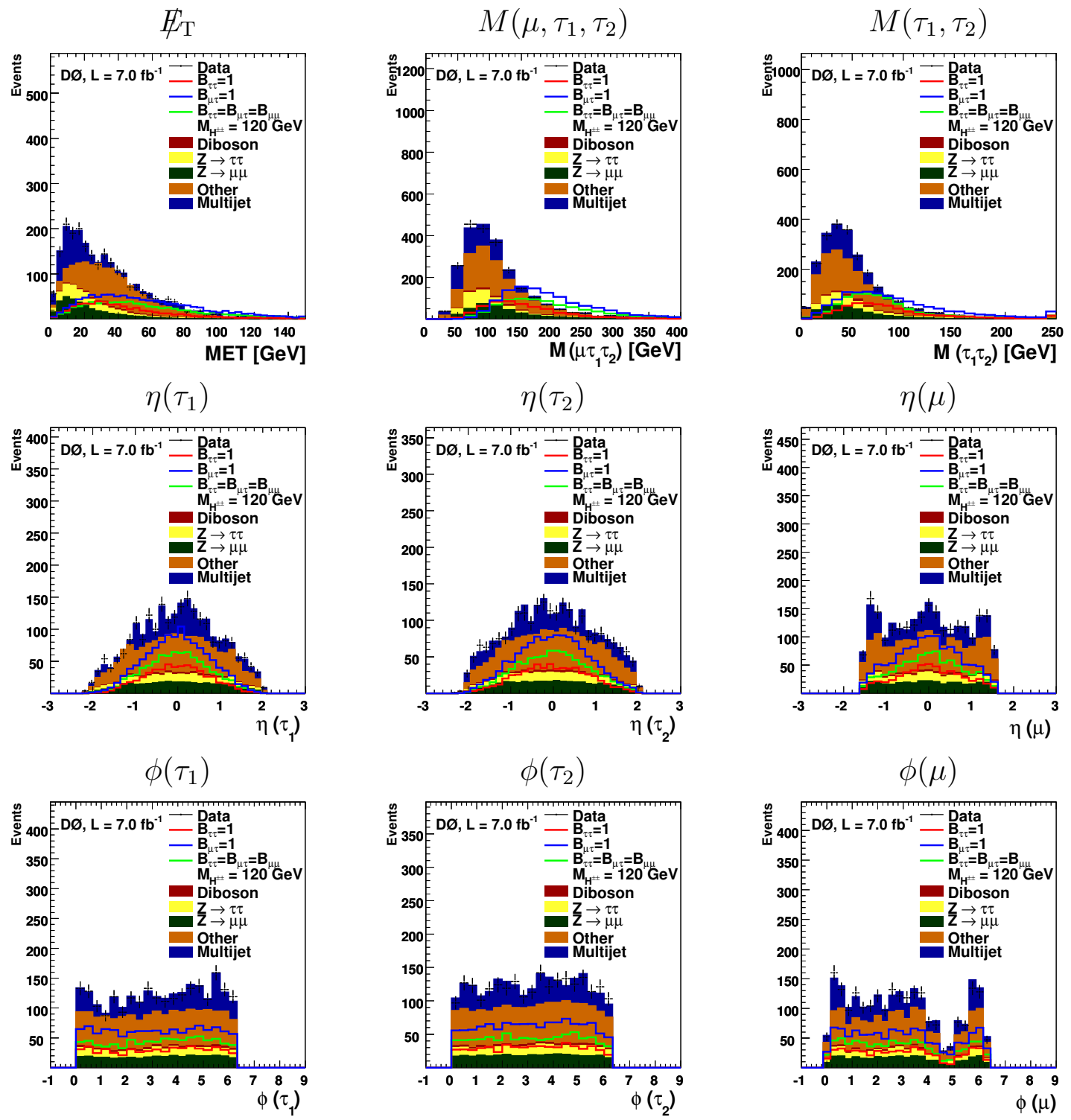

Figure 8.3: Data distributions at pre-selection compared to the sum of the expected backgrounds from MC simulations and multijet estimations. The signal distributions at pre-selection for a $M_{H^{ \pm \pm}}=120 \mathrm{GeV}$ left handed pair produced $H^{ \pm \pm}$boson for the cases where $B_{\tau \tau}=1, B_{\mu \tau}=1$, and $B_{\tau \tau}=B_{\mu \mu}=B_{\mu \tau}=\frac{1}{3}$. multiplied by a factor of 50, are superimposed. The 'Other' background sample contains the contributions from $Z \rightarrow e e, W+$ jets and $t \bar{t}$ production. 


\subsection{Instrumental background estimation}

To estimate the contribution from heavy flavour multijet events in the final selection sample of this analysis, instrumental background Method 1 (Section 7.8.1) is used, with instrumental background Method 2 (Section 7.8.2), being used as a cross check. The contribution from the $W+$ jet events is small and well modeled within the available statistics, therefore no reweighting of these events is performed [118]. The TTNN and SR samples represent the sample that the multijet events are being estimated for, and therefore their selection criteria is taken to match the final selection criteria, as given in Section 8.5.1. Therefore, the $N N_{\tau}^{t i g h t}$ requirement used for both instrumental background estimation methods is taken to be greater than 0.75 for all tau lepton types.

The two methods consistently give the prediction of multijet backgrounds at final selection to be zero [118]. An upper limit of 0.8 events is determined from the statistical uncertainties on the number of data and Monte Carlo events. This value is less than $2.5 \%$ of the total background contribution at final selection and is therefore neglected.

\subsubsection{Final selection requirements}

A series of additional selection criteria are applied to further reduce the background from $Z / \gamma^{*} \rightarrow \tau \tau, Z / \gamma^{*} \rightarrow \mu \mu, W+$ jets, and multijet events, and to enhance the signal region, creating a final selection sample. This section will describe the applied selection criteria along with their motivations. The efficiencies of these final selection requirements are shown in Table 8.3, where it can be clearly seen that it is the $N N_{\tau}$ requirement that removes a majority of the background events. 
1. $\Delta R\left(\tau_{1}, \tau_{2}\right)>0.7$

The $\Delta R\left(\tau_{1}, \tau_{2}\right)$ distribution, as shown in Figure 8.2, has a peak at low values from non isolated tau lepton candidates. Therefore, removing these events at low values will ensure that the tau lepton candidates are unique.

2. $\left|\eta_{\tau}\right|<1.5$ for both tau lepton candidates

The high values of $\left|\eta_{\tau}\right|$ are dominated by multijet events with a low concentration of signal events. Therefore removing these events helps to further select the signal-dominated region.

3. $|Q|=\left|\Sigma_{i} q_{i}\right|=1, i=\mu, \tau_{1}, \tau_{2}$

Requiring that two out of the three leptons have the same electric charge, as expected for the signal events, removes multijet background events. This is shown in Figure 7.11, where it can be seen that events with $|Q|=3$, where all three selected leptons have the same charge, are dominated by multijet events. The "charge flip" event rate, where leptons are reconstructed with the wrong charge, has been shown to be very small, of the order of $10^{-4}$ [108].

4. $N N_{\tau}>0.75$ for both tau lepton candidates

The $N N_{\tau}$ distribution peaks for the signal at one and at zero for the background. Requiring that tau candidates have high $N N_{\tau}$ removes the majority of the $W+$ jets and multijet events.

\subsubsection{Distributions at final selection}

The comparison of the data and MC events at final selection for the same selection of variables as described for the pre-selection (in Section 8.4.2) are shown in Figures 8.4 and 8.5. With all MC event corrections and normalizations applied, as covered in Section 7.4. Signal samples for the left handed doubly charged Higgs boson with a mass of $120 \mathrm{GeV}$, in the cases where $\mathcal{B}\left(H^{ \pm \pm} \rightarrow \tau \tau\right)=1$, 


\begin{tabular}{c|cccc} 
& \multicolumn{2}{|c}{ Data } & \multicolumn{2}{c}{ Background MC events } \\
Selection requirement & $N^{\text {evts }}$ & efficiency & $N^{\text {evts }}$ & efficiency \\
\hline Pre-selection & 14462 & $100 \%$ & 16493.7 & $100 \%$ \\
$\Delta R\left(\tau_{1}, \tau_{2}\right)>0.7$ & 2282 & $16 \%$ & 2361.8 & $14 \%$ \\
$\left|\eta_{\tau}\right|<1.5$ & 2154 & $15 \%$ & 2204.3 & $13 \%$ \\
$|Q|=1$ & 1545 & $11 \%$ & 1590.5 & $9 \%$ \\
$N N_{i}>0.75$ & 1295 & $9 \%$ & 1334.8 & $8 \%$ \\
& 22 & $0.15 \%$ & 27.6 & $0.17 \%$
\end{tabular}

Table 8.3: The number of events passing each final selection requirement and the corresponding efficiencies for both data and background $\mathrm{MC}$ events, given in percentage of the number of pre-selection events passing each requirement.

$\mathcal{B}\left(H^{ \pm \pm} \rightarrow \mu \tau\right)=1$ and the equal $\mathcal{B}$ case where $\mathcal{B}\left(H^{ \pm \pm} \rightarrow \tau \tau\right)=\mathcal{B}\left(H^{ \pm \pm} \rightarrow \mu \mu\right)$

$=\mathcal{B}\left(H^{ \pm \pm} \rightarrow \mu \tau\right)=\frac{1}{3}$, are superimposed. The number of events predicted from the MC simulation and in data, corresponding to the final selection requirements are given in Table 8.4 .

\subsection{Signal sample comparison}

Figure 8.6 shows a comparison of the generated signal samples for both a left and right handed doubly charged Higgs boson, of mass $120 \mathrm{GeV}$ at final selection. The cross section of the right handed $H^{ \pm \pm}$boson is lower, but after reconstruction no significant kinematic differences are observed between the distributions for the left and right handed $H^{ \pm \pm}$boson.

\subsection{Results}

\subsubsection{Benchmark points for limiting setting}

The limits are set for the five benchmark points, which correspond to specific $H^{ \pm \pm}$boson decay channels of interest. The benchmark points are summarized in 

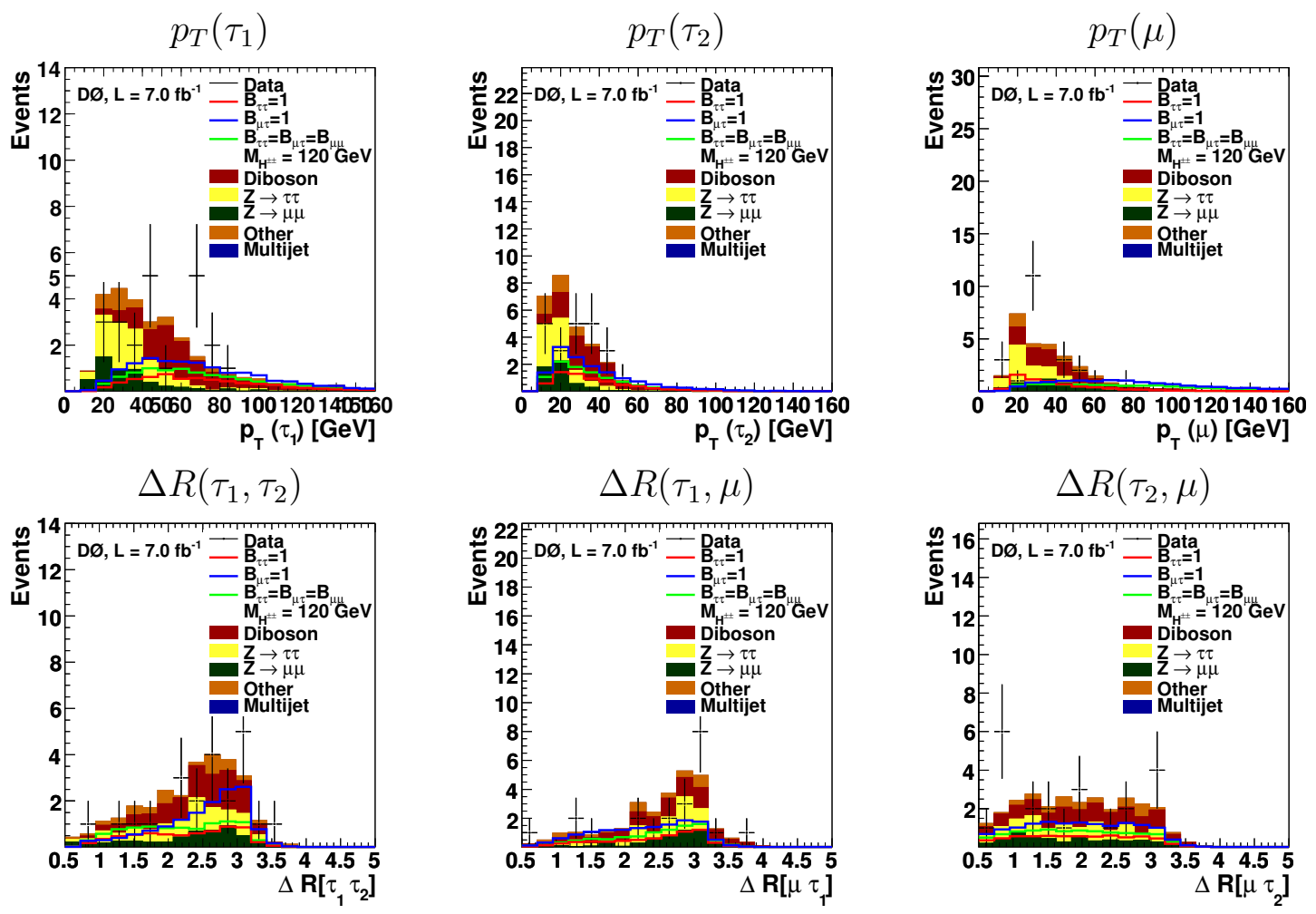

$N_{\mu}$

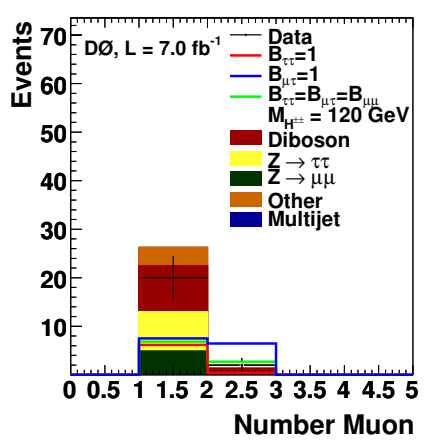

$N_{\tau}$

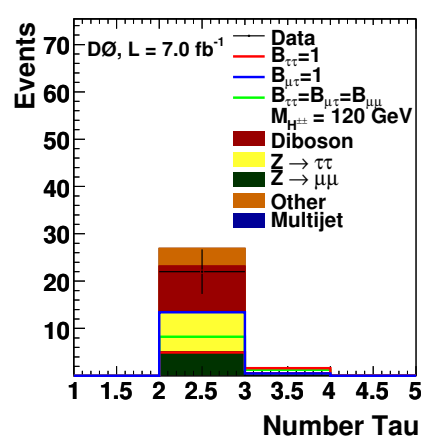

$M_{T}$

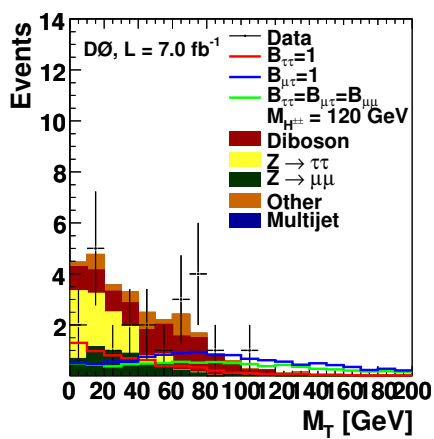

Figure 8.4: Data distributions at final selection compared to the sum of the expected backgrounds from MC simulations and multijet estimations.. The signal distributions at final selection for a $M_{H^{ \pm \pm}}=120 \mathrm{GeV}$ left handed pair produced $H^{ \pm \pm}$boson for the cases where $B_{\tau \tau}=1, B_{\mu \tau}=1$, and $B_{\tau \tau}=B_{\mu \mu}=B_{\mu \tau}=\frac{1}{3}$. The 'Other' background sample contains the contributions from $Z \rightarrow e e, W+$ jets and $t \bar{t}$ production. 

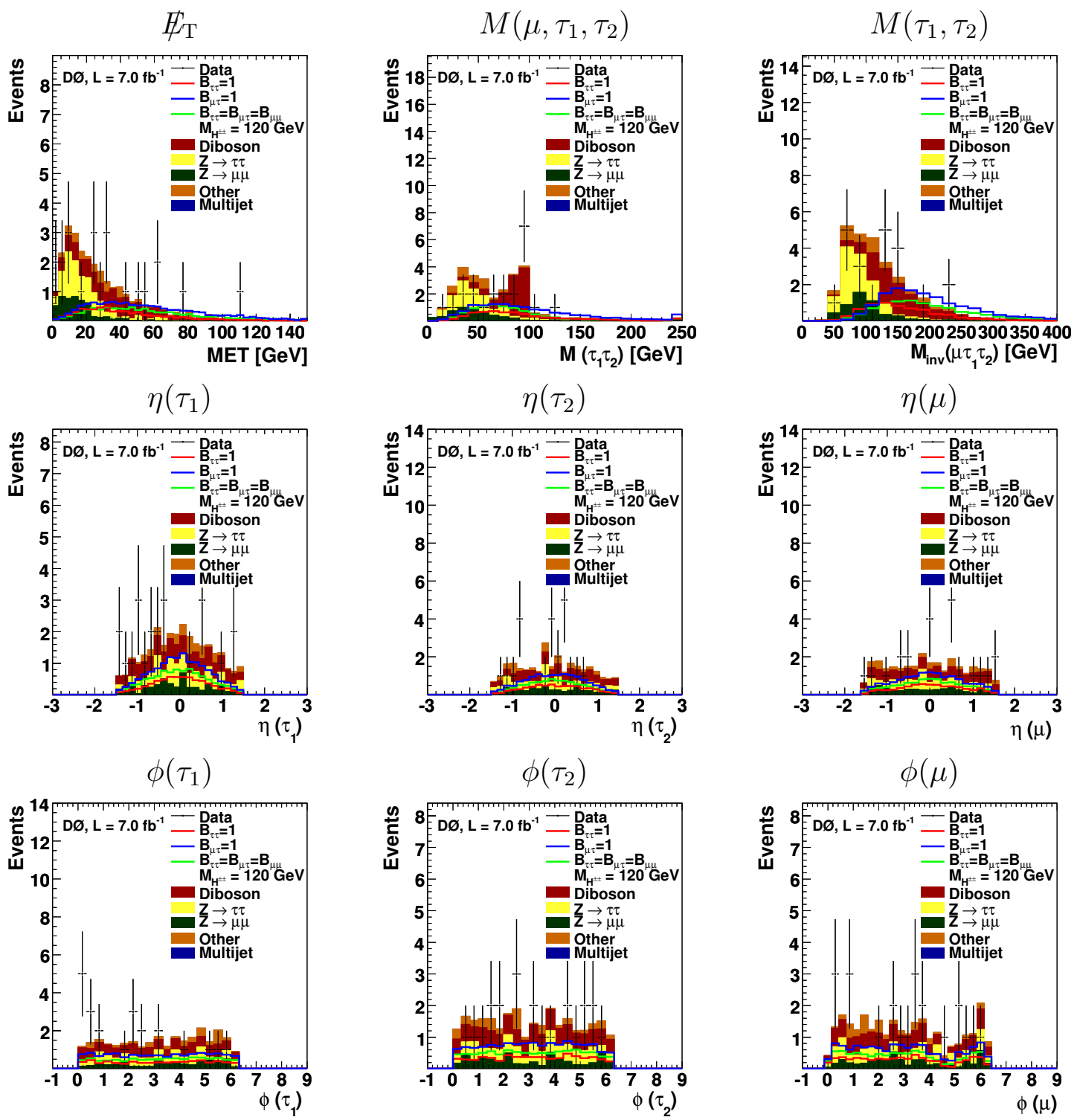

Figure 8.5: Data distributions at final selection compared to the sum of the expected backgrounds from MC simulations and multijet estimations. The signal distributions at final selection for a $M_{H^{ \pm \pm}}=120 \mathrm{GeV}$ left handed pair produced $H^{ \pm \pm}$boson for the cases where $B_{\tau \tau}=1, B_{\mu \tau}=1$, and $B_{\tau \tau}=B_{\mu \mu}=B_{\mu \tau}=\frac{1}{3}$. The 'Other' background sample contains the contributions from $Z \rightarrow e e, W+$ jets and $t \bar{t}$ production. 

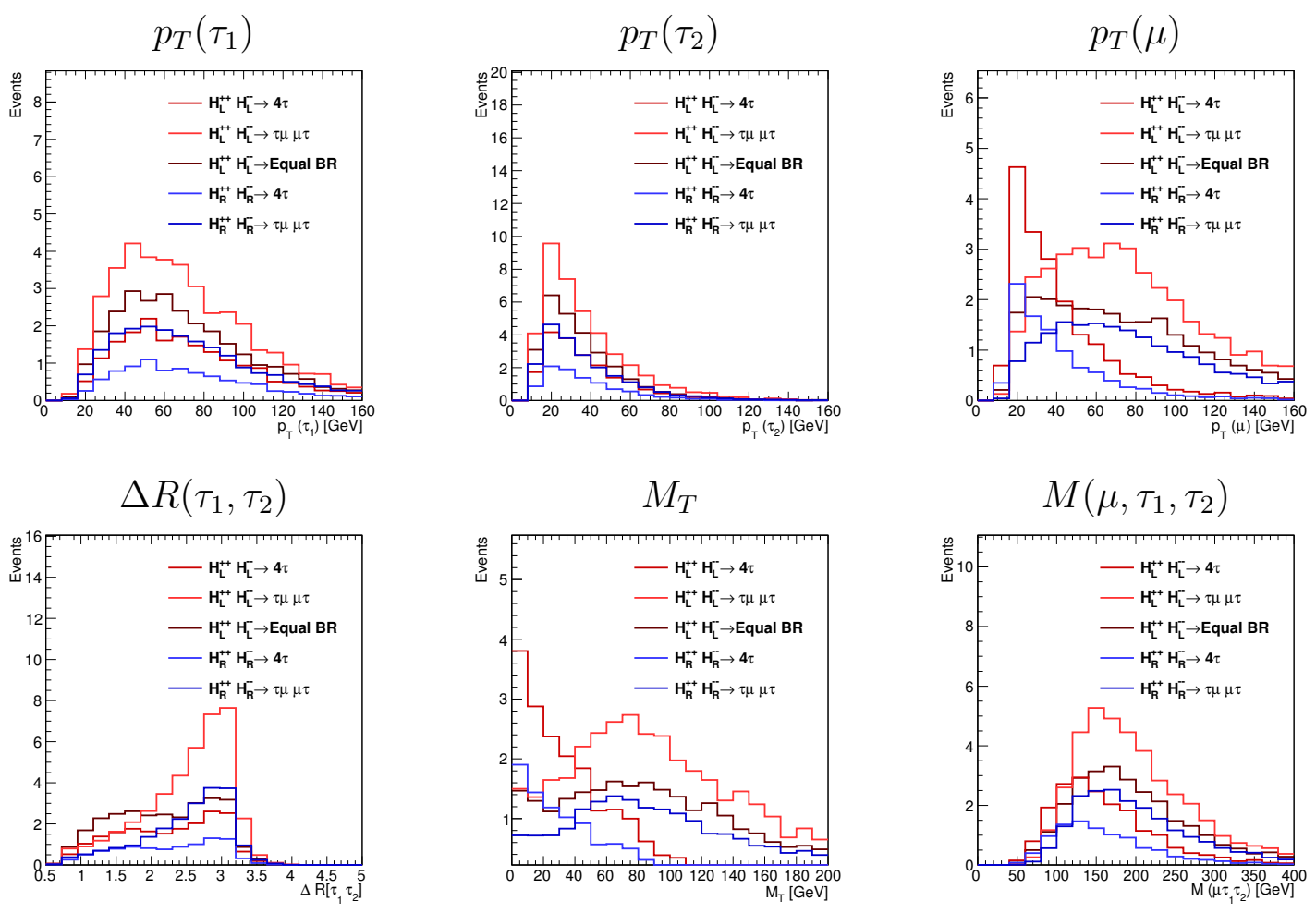

Figure 8.6: Comparison of the left (red) and right handed (blue) $H^{ \pm \pm}$boson signals, scaled by a factor of 3 . Samples are shown for $\mathcal{B}\left(H^{ \pm \pm} \rightarrow \tau \tau\right)=1$ $\left(H^{ \pm \pm} H^{ \pm \pm} \rightarrow 4 \tau\right)$ and $\mathcal{B}\left(H^{ \pm \pm} \rightarrow \mu \tau\right)=1\left(H^{ \pm \pm} H^{ \pm \pm} \rightarrow \mu \tau \mu \tau\right)$. For a left handed $H^{ \pm \pm}$Higgs boson the decay with equal branching ratios to $\mu \tau, \tau \tau$, and $\mu \mu$ lepton pairs is also shown, $\mathcal{B}\left(H^{ \pm \pm} \rightarrow \tau \tau\right)=\mathcal{B}\left(H^{ \pm \pm} \rightarrow \mu \mu\right)=\mathcal{B}\left(H^{ \pm \pm} \rightarrow \mu \tau\right)=\frac{1}{3}$.

Table 8.7.1, along with the MC samples used for each generated benchmark point. For points (2) and (3), a sample where $\mathcal{B}\left(H^{ \pm} \rightarrow \mu \mu\right)=1$ is required. This is not a decay mode that this analysis is sensitive to due to the requirement to have two tau leptons in the final state. Therefore, a previous $D \varnothing$ search studying the pair production of $H^{ \pm \pm}$bosons decaying with $\mathcal{B}\left(H^{ \pm \pm} \rightarrow \tau \tau\right), H^{++} H^{--} \rightarrow 4 \mu$ [37], with an integrated luminosity of $L=1.1 \mathrm{fb}^{-1}$, is used for these benchmark points. The $H^{++} H^{--} \rightarrow 4 \mu$ analysis was re-analysed within the limit setting porton of this analyses framework, using the original RooT trees [119]. This data and MC sample will be referred to as $4 \mu$. Details of the $H^{++} H^{--} \rightarrow 4 \mu$ analysis are given 


\begin{tabular}{l|c|c} 
& Pre-selection & Final selection \\
\hline \hline Data & 2283 & 22 \\
$Z / \gamma^{*} \rightarrow \tau \tau$ & $243.9 \pm 34.1$ & $8.16 \pm 1.14$ \\
$Z / \gamma^{*} \rightarrow \mu \mu$ & $350.0 \pm 49.0$ & $5.09 \pm 0.71$ \\
$Z / \gamma^{*} \rightarrow \mu \mu$ & $1.0 \pm 0.1$ & $0.30 \pm 0.04$ \\
$W+$ jets & $979.0 \pm 137.1$ & $2.90 \pm 0.41$ \\
$t \bar{t}$ & $85.2 \pm 12.5$ & $0.64 \pm 0.09$ \\
Diboson & $57.9 \pm 9.5$ & $10.48 \pm 1.72$ \\
Multijet & $594.6 \pm 237.8$ & $0.00 \pm 0.76$ \\
Total Bkg & $2361.8 \pm 467.7$ & $27.58 \pm 4.87$ \\
\hline Signal $(\mathbf{1 2 0} \mathbf{G e V})$ & & \\
$\mathcal{B}_{\tau \tau}=1$ & $11.25 \pm 1.53$ & $6.57 \pm 0.89$ \\
$\mathcal{B}_{\tau \tau}=\mathcal{B}_{\mu \mu}=\mathcal{B}_{\mu \tau}=1 / 3$ & $16.52 \pm 2.25$ & $9.54 \pm 1.30$ \\
$\mathcal{B}_{\mu \tau}=1$ & $23.58 \pm 3.21$ & $13.93 \pm 1.89$
\end{tabular}

Table 8.4: Prediction from MC events and multijet estimation methods compared to the observation in data at both pre- and final selection. Systematic uncertainties on the MC and multijet are given. The contribution from statistical uncertainties is taken to be negligible and not included.

in Section 8.7.2. For point (2) the generated MC signal samples for a $H^{ \pm \pm}$boson with $\mathcal{B}\left(H^{ \pm \pm} \rightarrow \tau \tau\right)$, with $\mathcal{B}\left(H^{ \pm \pm} \rightarrow \mu \mu\right)$, and with the mixed decay where the $H^{ \pm \pm}$decays to both $\tau$ leptons and muon, must be and combined correctly. The samples must be mixed with the following fractions, $f$.

- $f=\mathrm{B}_{\tau \tau}^{2}$ for the $4 \tau$ MC sample,

- $f=\left(1-\mathrm{B}_{\tau \tau}\right)^{2}$ for the $4 \mu \mathrm{MC}$ sample,

- $f=2 \mathrm{~B}_{\tau \tau}\left(1-\mathrm{B}_{\tau \tau}\right)$ for the $2 \tau 2 \mu \mathrm{MC}$ sample.

The generated MC signal samples are defined in Table 8.1. Point (3) consists purely of the $4 \mu$ sample re-analysised in the framework of this analysis. For points (1), (4), and (5) the integrated luminosity corresponds to $L=7.0 \mathrm{fb}^{-1}$, for point (2) a combination of an integrated luminosity of $L=7.0 \mathrm{fb}^{-1}$ and an integrated luminosity of $L=1.1 \mathrm{fb}^{-1}$ is used and for point (3) an integrated luminosity of $L=1.1 \mathrm{fb}^{-1}$ is used. 


\begin{tabular}{c|c|c|c|c}
$\mathrm{BP}$ & $\mathcal{B}\left(H^{ \pm \pm} \rightarrow \tau \tau\right)$ & $\mathcal{B}\left(H^{ \pm \pm} \rightarrow \mu \mu\right)$ & $\mathcal{B}\left(H^{ \pm \pm} \rightarrow \mu \tau\right)$ & Samples Used \\
\hline 1 & $100 \%$ & $0 \%$ & $0 \%$ & $4 \tau$ \\
\hline & $90 \%$ & $10 \%$ & $0 \%$ & $4 \tau, 2 \tau 2 \mu, 4 \mu$ \\
& $80 \%$ & $20 \%$ & $0 \%$ & $4 \tau, 2 \tau 2 \mu, 4 \mu$ \\
& $70 \%$ & $30 \%$ & $0 \%$ & $4 \tau, 2 \tau 2 \mu, 4 \mu$ \\
& $60 \%$ & $40 \%$ & $0 \%$ & $4 \tau, 2 \tau 2 \mu, 4 \mu$ \\
2 & $50 \%$ & $50 \%$ & $0 \%$ & $4 \tau, 2 \tau 2 \mu, 4 \mu$ \\
& $40 \%$ & $60 \%$ & $0 \%$ & $4 \tau, 2 \tau 2 \mu, 4 \mu$ \\
& $30 \%$ & $70 \%$ & $0 \%$ & $4 \tau, 2 \tau 2 \mu, 4 \mu$ \\
& $20 \%$ & $80 \%$ & $0 \%$ & $4 \tau, 2 \tau 2 \mu, 4 \mu$ \\
& $10 \%$ & $90 \%$ & $0 \%$ & $4 \tau, 2 \tau 2 \mu, 4 \mu$ \\
\hline 3 & $0 \%$ & $100 \%$ & $0 \%$ & $4 \mu$ \\
\hline 4 & $0 \%$ & $0 \%$ & $100 \%$ & $\mu \tau$ \\
\hline 5 & $1 / 3$ & $1 / 3$ & $1 / 3$ & Equal $\mathcal{B}$
\end{tabular}

Table 8.5: Summary of the five benchmark points, BP , defined. The branching fraction into $\tau \tau, \mu \mu$, or $\mu \tau$ lepton pairs is given, as well as the generated signal samples used for each point. The equal $\mathcal{B}$ signal corresponds to $\mathcal{B}\left(H^{++} \rightarrow \tau \tau\right)=$ $\mathcal{B}\left(H^{++} \rightarrow \mu \mu\right)=\mathcal{B}\left(H^{++} \rightarrow \mu \tau\right)=\frac{1}{3}$.

\subsubsection{Summary of the $H^{++} H^{--} \rightarrow 4 \mu$ analysis}

The $H^{++} H^{--} \rightarrow 4 \mu$ analysis [37] is preformed using an integrated luminosity of $L=1.1 \mathrm{fb}^{-1}$, with the following selection requirements. Three isolated muons are selected and the azimuthal angle $\Delta \phi$ between at least one pair of muons must be $\Delta \phi<2.5$. The final discriminant is the invariant mass, $M(\mu, \mu)$, between the two highest $p_{T}$ muons. Three events are found, with an expected background of $2.3 \pm$ 0.2 events. The background events are mainly $(\simeq 90 \%)$ from diboson processes, with a small multijet contribution. The invariant mass $M(\mu, \mu)$ between the two highest $p_{T}$ muons used for the final discriminant, is shown in Figure 8.7.

\subsubsection{Final discriminants}

To produce a final discriminant distribution that will be used to set the cross section limits for the $H^{ \pm \pm}$boson, the events at final selection are split into four 


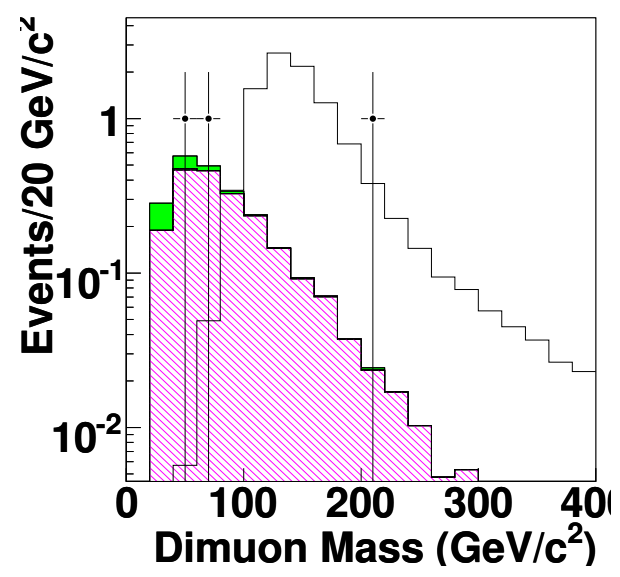

Figure 8.7: The invariant mass $M(\mu, \mu)$ between the two highest $p_{T}$ muons, used as the final discriminate in the $H^{++} H^{--} \rightarrow 4 \mu$ [37]. The diboson background is shown in pink, the multijet in green, and the $\mathcal{B}\left(H^{++} \rightarrow \mu \mu\right)=1$ signal is represented by the open histogram.

orthogonal sub-samples. Two of these samples depend on the charge correlation between the electric charges of the muon and the tau lepton candidates and two depend on the number of leptons. The two leptons with the same charge are assumed to originate from the same $H^{ \pm \pm}$boson decay, with the charge flip of the lepton assumed to be negligible. The four channels are defined as:

- TT channel: Requires exactly two tau leptons and one muon and that the two tau lepton candidates are of the same electric charge, $N_{\tau}=2, N_{\mu}=1$ and $q\left(\tau_{1}\right)=q\left(\tau_{2}\right)$.

- TM channel: Requires exactly two tau leptons and one muon and either of the two tau lepton candidates and the muon are of the same electric charge, $N_{\tau}=2, N_{\mu}=1$ and $q\left(\tau_{1}\right) \neq q\left(\tau_{2}\right)$.

- T3 channel: Requires events to have three hadronically decaying tau leptons and one muon, with no additional requirements on the electric charges of the leptons, $N_{\tau}=3$ and $N_{\mu}=1$. 
- M2 channel: Requires events to have two muons and two tau leptons, with no additional requirements on the electric charges of the leptons, $N_{\tau}=2$ and $N_{\mu}=2$.

For each of these four independent channels a separate final discriminant is defined. For the two same sign channels (TT and TM) the most discriminating variable is determined to be the invariant mass of the two tau leptons. For the T3 and M2 channels due to the low statistics in these channels, only the number of events passing the final selection is used, i.e. no shape information is used in the limit setting procedure and the limits are set in a single bin as a counting experiment. These distributions can be seen in Figure 8.8 and the number of predicted MC and data events in each channel in Table 8.6.

The four channels have different kinematics which can be utilised to achieve increased sensitivity to the different benchmark points. The TT and T3 channels are most sensitive to the signal sample for a $H^{ \pm \pm}$boson with $\mathcal{B}\left(H^{++} \rightarrow \tau \tau\right)$ and with $\mathcal{B}\left(H^{++} \rightarrow \tau \tau\right)=\mathcal{B}\left(H^{++} \rightarrow \mu \mu\right)=\mathcal{B}\left(H^{++} \rightarrow \mu \tau\right)=\frac{1}{3}$, whereas the main signal contribution in the TM and M2 channels is when the $H^{ \pm \pm}$bosons with $\mathcal{B}\left(H^{++} \rightarrow \mu \tau\right)$.

\subsection{Systematic uncertainties}

The systematic uncertainties common to both analysis described in thesis are described in Section 7.10. The uncertainties specific for this analysis are described in this section. All systematic uncertainties applied in this analysis are summarized in Table 8.7. For the $H^{++} \rightarrow 4 \mu$ sample the uncertainties as derived for the original publication are used. Detailed description is given in ref. [37] and will not be repeated here:

- The uncertainty from muon identification is taken to be $2 \%$ and $6 \%$ for 


\begin{tabular}{l|c|c|c|c} 
& $\mathrm{TT}$ & $\mathrm{TM}$ & $\mathrm{T} 3$ & $\mathrm{M} 2$ \\
\hline \hline Data & 5 & 15 & 0 & 2 \\
$Z / \gamma^{*} \rightarrow \tau \tau$ & $3.37 \pm 0.47$ & $4.75 \pm 0.67$ & $0.04 \pm 0.00$ & $<0.01$ \\
$Z / \gamma^{*} \rightarrow \mu \mu$ & $2.24 \pm 0.31$ & $2.54 \pm 0.36$ & $0.08 \pm 0.01$ & $0.2 \pm 0.03$ \\
$Z / \gamma^{*} \rightarrow \mu \mu$ & $<0.01$ & $0.28 \pm 0.04$ & $0.01 \pm 0.00$ & $<0.01$ \\
$W+$ jets & $1.08 \pm 0.15$ & $1.81 \pm 0.28$ & $<0.01$ & $<0.01$ \\
$t \bar{t}$ & $0.27 \pm 0.04$ & $0.28 \pm 0.05$ & $0.08 \pm 0.01$ & $<0.01$ \\
Diboson & $0.49 \pm 0.08$ & $8.50 \pm 1.40$ & $0.40 \pm 0.07$ & $1.06 \pm 0.17$ \\
Multijet & $0.00 \pm 0.17$ & $0.00 \pm 0.52$ & $0.00 \pm 0.03$ & $0.00 \pm 0.07$ \\
Total Bkg & $7.47 \pm 1.22$ & $18.17 \pm 3.32$ & $0.61 \pm 0.12$ & $1.28 \pm 0.27$ \\
\hline Signal 120 GeV & & & & \\
$B_{\tau \tau}=1$ & $1.44 \pm 0.20$ & $3.08 \pm 0.42$ & $1.64 \pm 0.24$ & $0.40 \pm 0.05$ \\
$B_{\tau \tau}=B_{\mu \mu}=B_{\mu \tau}=1 / 3$ & $2.48 \pm 0.34$ & $3.12 \pm 0.42$ & $1.23 \pm 0.17$ & $2.63 \pm 0.40$ \\
$B_{\mu \tau}=1$ & $0.28 \pm 0.04$ & $6.83 \pm 0.93$ & $0.37 \pm 0.06$ & $6.28 \pm 0.86$
\end{tabular}

Table 8.6: Prediction from the simulated MC and data driven multijet method compared to the observation in data for the four final discriminant channels. Systematic uncertainties on the simulated MC and multijet are given. The contribution from statistical uncertainties is considered to be negligible and not included.

backgrounds and signal, respectively.

- The uncertainty due to electric charge misidentification is taken at $20 \%$.

- The uncertainty on the integrated luminosity is $6.1 \%$.

- The uncertainty on the signal cross section due to the PDF is $4 \%$.

- A $5 \%$ uncertainty on the diboson cross section from the normalization of the MC cross section to the NNLO cross section is used.

\section{Analysis specific uncertainties:}

- The uncertainty for the muon identification depends on the quality requirements used, as described in Section 7.10.

- For the "Medium" muon quality, an uncertainty of $1.1 \%$ has been determined. In addition, $20 \%$ of events have a muon with a $p_{T}$ of 
TT Channel

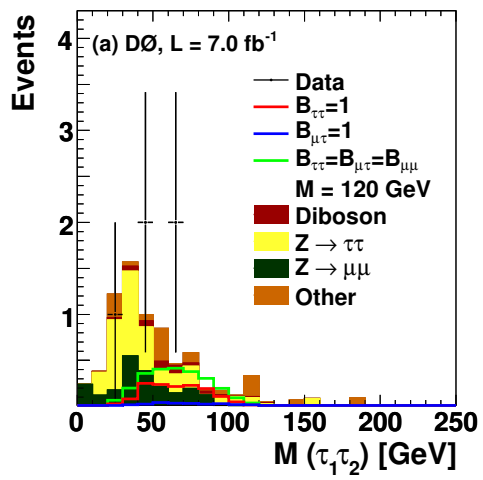

T3 Channel

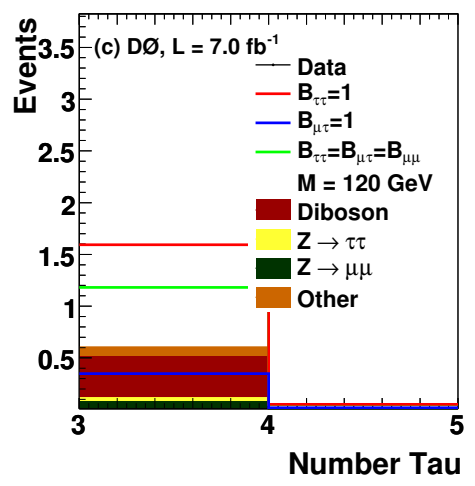

TM Channel

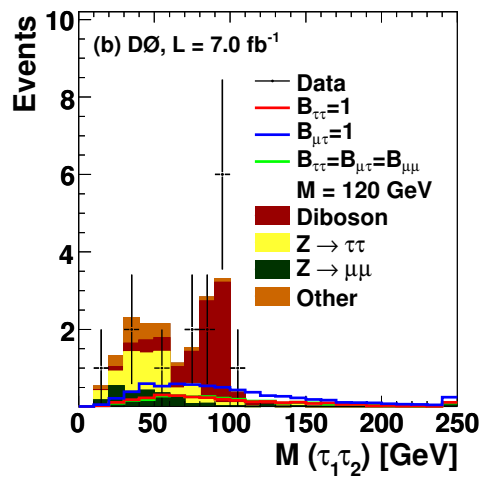

M2 Channel

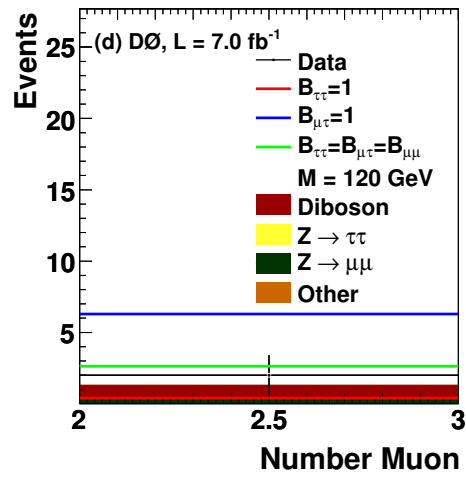

Figure 8.8: The comparison of data to expected background for the discriminating variables. For the TT and TM channels this is the invariant mass of the two tau candidates, for $\mathbf{T} 3$ the number of tau leptons, and $\mathbf{M} 2$ the number of muons. The signal distribution for a left-handed $H^{ \pm \pm}$boson in the cases where $\mathrm{B}_{\tau \tau}=$ $1, \mathrm{~B}_{\mu \tau}=1$ and $\mathrm{B}_{\tau \tau}=\mathrm{B}_{\mu \mu}=\mathrm{B}_{\mu \tau}=\frac{1}{3}$ with $M\left(H^{ \pm \pm}\right)=120 \mathrm{GeV}$ is superimposed.

less than $20 \mathrm{GeV}$, which have an additional $2 \%$, leading to a total uncertainty of $1.3 \%$.

- For the "TrackMedium" track quality the uncertainty is $1.1 \%$

- For the "NPTight" isolation requirements, the uncertainty is $0.9 \%$. For the $20 \%$ of events which have a muon with a $p_{T}<20 \mathrm{GeV}$ a additional $2 \%$ uncertainty is needed, which leads to a total uncertainty of $1.2 \%$.

The total uncertainty, combined in quadrature, is $2.9 \%$. 
- The uncertainty on the $N N_{\tau}$, as covered in Section 7.4.6, is both $N N_{\tau}$ specific and $N N_{\tau}$ requirement specific. For the $N N_{2010}$ and a $0.75 N N_{\tau}$ requirement the uncertainty has been determined to be $4 \%$ per tau for Type-1 tau leptons and 7\% per tau lepton for Type-2 tau leptons [103]. This is added in quadrature with the uncertainty on the tracking efficiency of $1.4 \%$ and the uncertainty on the tau energy scale of $1.0 \%$. As we do not differentiate between tau leptons types, an overall $10 \%$ systematic is applied for the channels with two tau leptons in the final state (TT, TM, and M2 channels) and 12\% for the T3 channels with three tau leptons in the final state.

- The uncertainty on the signal cross sections due to the uncertainty on the PDF set used is $4 \%$ as calculated by Ref. [91].

\begin{tabular}{l|ccccc|cc}
\multirow{2}{*}{$\begin{array}{l}\text { Source of } \\
\text { uncertainty }\end{array}$} & \multicolumn{7}{|c}{ Relative error on each samples [\%] } \\
\cline { 2 - 8 } & $Z / \gamma^{*}$ & $W+$ jets & $t \bar{t}$ & Diboson & Signal & Diboson & Signal \\
\hline \hline Cross section & 6.0 & 6.0 & 10.0 & 7.0 & - & 5.0 & - \\
Luminosity & 6.1 & 6.1 & 6.1 & 6.1 & 6.1 & 6.1 & 6.1 \\
Muon Id & 2.9 & 2.9 & 2.9 & 2.9 & 2.9 & 2.0 & 6.0 \\
Tau Track & 1.4 & 1.4 & 1.4 & 1.4 & 1.4 & - & - \\
match per $\tau$ & & & & & & & - \\
Tau ID per $\tau$ & $7 / 4$ & $7 / 4$ & $7 / 4$ & $7 / 4$ & $7 / 4$ & - & - \\
$(\tau$-Type-1/2) & & & & & & & - \\
Tau lepton & 1.0 & 1.0 & 1.0 & 1.0 & 1.0 & - & - \\
energy scale & & & & & & & - \\
Trigger & 5.0 & 5.0 & 5.0 & 5.0 & 5.0 & - & - \\
PDF & - & - & - & - & 4.0 & - & 4.0 \\
Charge & - & - & - & - & - & 20.0 & 20.0 \\
misidentification & & & & & & &
\end{tabular}

Table 8.7: Summary of the sources of systematic errors for both the signal and background samples as described in the text. The uncertainties for the $H^{++} \rightarrow 4 \mu$ sample are also given, as determined by [37]. 


\subsection{Cross section limits}

As no significant excess in data over the total predicted background is observed, limits are set on the cross section for the pair production of both the left and right handed doubly charged Higgs bosons. Except for benchmark point (5) as right handed $H^{ \pm \pm}$bosons are not applicable. The calculation is performed by the COLLIE package [113] (V00-04-09), as described in detail in Section 7.11. The same procedure is performed for both the left and right handed doubly charged Higgs bosons. The expected limits are determined for six $H^{ \pm \pm}$boson masses (100, 120, 140, 160, 180 and $200 \mathrm{GeV}$ ) for the left handed $H^{ \pm \pm}$boson, and for seven masses for the right handed states (90, 100, 120, 140, 160, 180 and $200 \mathrm{GeV}$ ), due to the lower predicted cross section.

The four independent channels, TT, TM, T3, and M2 (Section 8.7.3), with their respective final discriminants, are used as inputs to COLLIE, as well as the final discriminant from the $H^{++} H^{--} \rightarrow 4 \mu$ analysis. For benchmark points the signal samples for the four independent channels and the $\mathrm{H}^{++} \mathrm{H}^{--} \rightarrow 4 \mu$ analysis are reweighted to match the branching ratios of $H^{ \pm \pm}$boson for that point, as given in Table 8.5. All the systematic uncertainties described in Section 7.10 and Section 8.8 are included in the calculation. A summary of the lower mass exclusion limits for the five benchmark points is listed in Table 8.12.

The upper limits on the product of the cross section and the branching ratio for benchmark points (1), (2) and (3) are listed in Tables 8.8 and 8.9 for the left and right handed doubley charged Higgs bosons, respectively. The distribution of the observed and expected limits at 95\% C.L. and the corresponding LLR distributions, as a function of the $H^{ \pm \pm}$boson mass are shown in Figures 8.10 and 8.11, for the left and right handed $H^{ \pm \pm}$boson, respectively. These are shown for three cases of the $H^{ \pm \pm}$boson decay, (a) benchmark point (1), $\mathcal{B}\left(H^{ \pm \pm} \rightarrow \tau \tau\right)$ $=1$, (b) one specific case of benchmark point $(2) \mathcal{B}\left(H^{ \pm \pm} \rightarrow \tau \tau\right)=\mathcal{B}\left(H^{ \pm \pm} \rightarrow\right.$ 

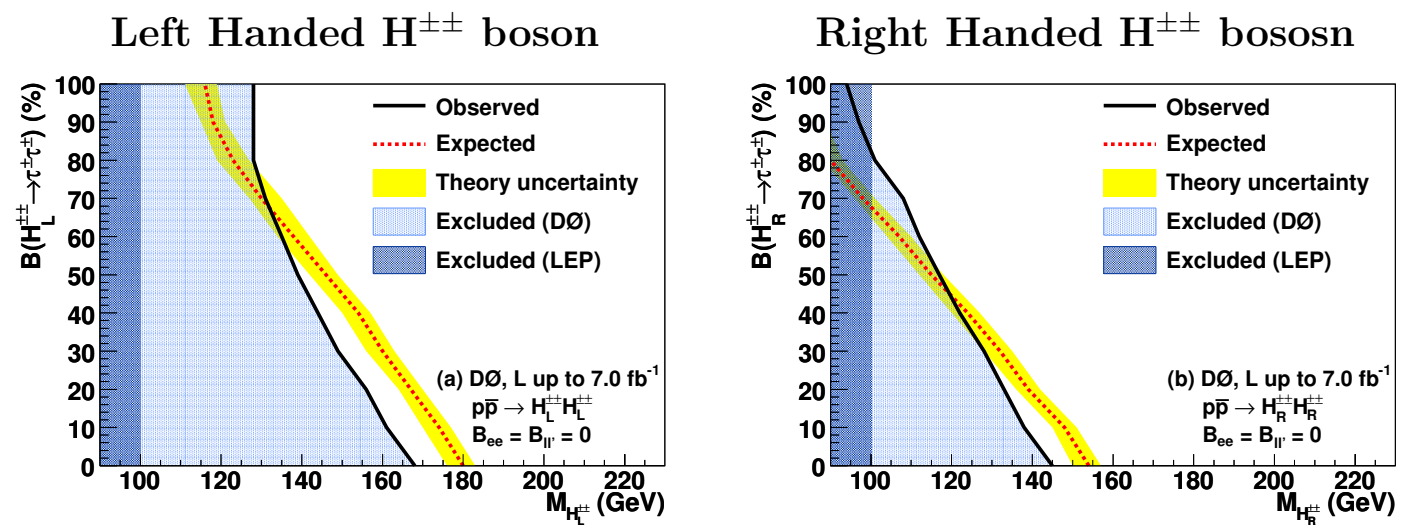

Figure 8.9: Lower mass exclusion regions for $\mathrm{B}\left(H^{ \pm \pm} \rightarrow \tau \tau\right)+\mathrm{B}\left(H^{ \pm \pm} \rightarrow \mu \mu\right)=1$, for the left handed doubly charged Higgs boson (left) and the right handed doubly charged Higgs boson (right). The observed and expected limits are shown by the black and red lines respectively. The yellow band corresponds to the uncertainty of the expected limit due to the uncertainty on the calculated NLO theoretical cross section [115]. The region exclude by LEP is shown in dark blue and the new regions exclude by these limits in light blue.

$\mu \mu)=0.5$, and (c) benchmark point $(3) \mathcal{B}\left(H^{ \pm \pm} \rightarrow \mu \mu\right)=1$. The blue band corresponds to the calculated NLO theoretical cross sections with its associated uncertainty [115], as discussed in Section 8.3.

The mass regions excluded by the cross section limits for when $\mathcal{B}\left(H^{ \pm \pm} \rightarrow \tau \tau\right)$ $+\mathcal{B}\left(H^{ \pm \pm} \rightarrow \tau \tau\right)=1$ as a function of the $\mathcal{B}\left(H^{ \pm \pm} \rightarrow \tau \tau\right)$, are shown in Figure 8.9 for both the left and right handed doubly charged Higgs bosons.

The determined observed and expected upper limits on the product of the cross section and the branching fraction, at 95\% C.L., are listed in Table 8.10 for benchmark point (4) and Table 8.11 for benchmark point (5). The distribution of the observed and expected upper limits at 95\% C.L. and the corresponding LLR distributions, are shown in Figure 8.12 for benchmark point (4) and in Figure 8.13 for benchmark point (5). 

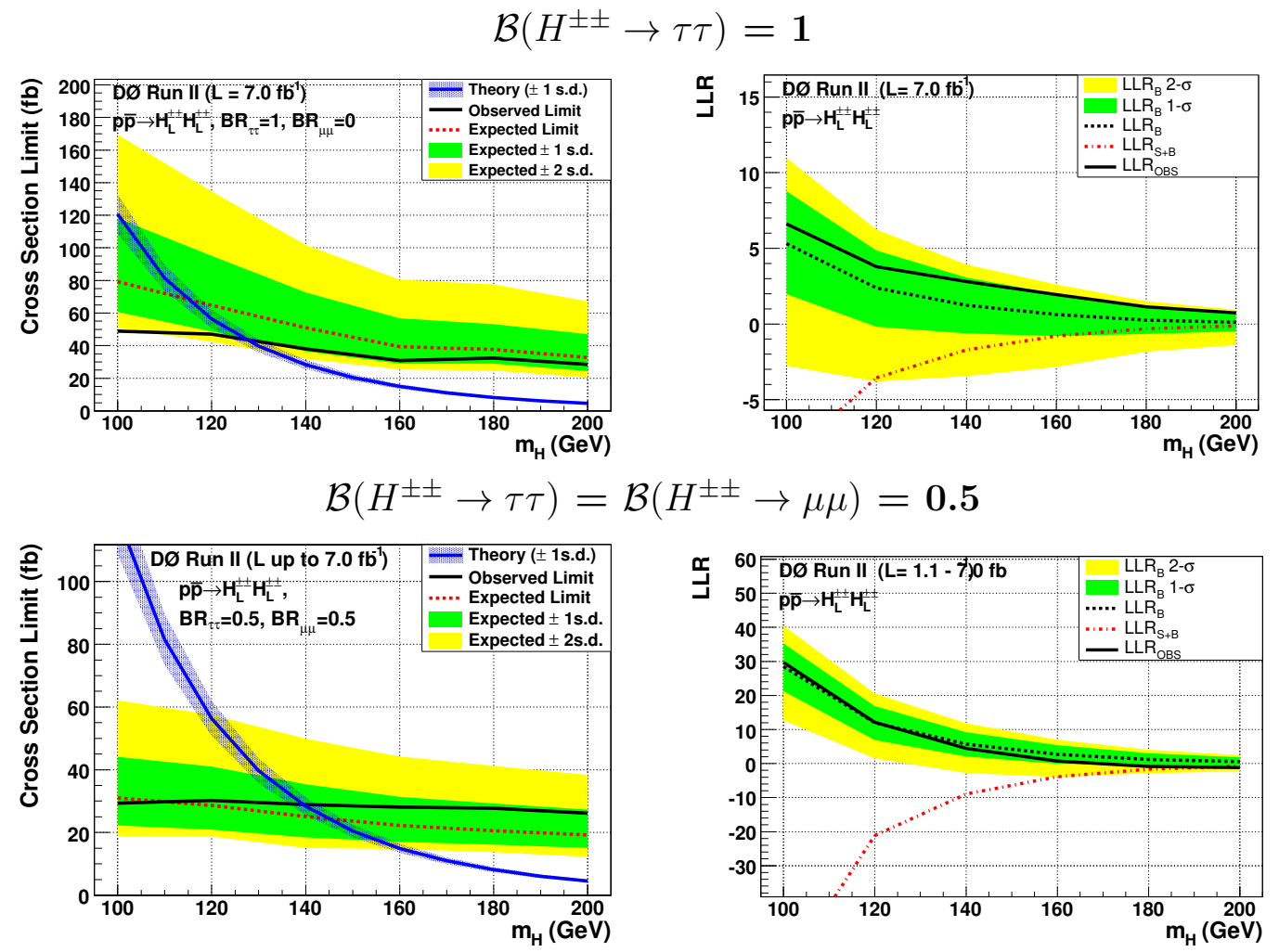

$$
\mathcal{B}\left(H^{ \pm \pm} \rightarrow \mu \mu\right)=\mathbf{1}
$$
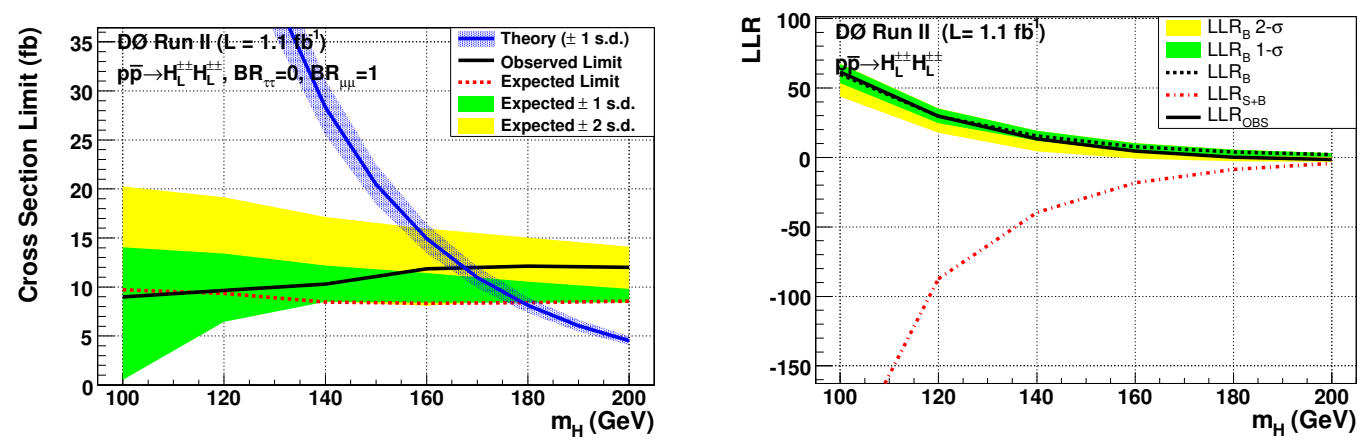

Figure 8.10: Expected and observed cross section limits, at 95\% C.L, and the corresponding LLR distributions for a left handed doubly charged Higgs boson, for benchmark points (1) (top), a specific case of benchmark point (2), $\mathcal{B}\left(H^{ \pm \pm} \rightarrow \tau \tau\right)$ $=\mathcal{B}\left(H^{ \pm \pm} \rightarrow \mu \mu\right)=0.5$ (middle), and benchmark point (3) (bottom). The observed and expected cross section limits are shown by the black and red lines, respectivaly. The yellow and green bands correspond to the $\pm 1 \sigma$ and $\pm 2 \sigma$ variation on the expected limits. The blue band in the limit plot shows the theoretical cross section and its uncertainty. 

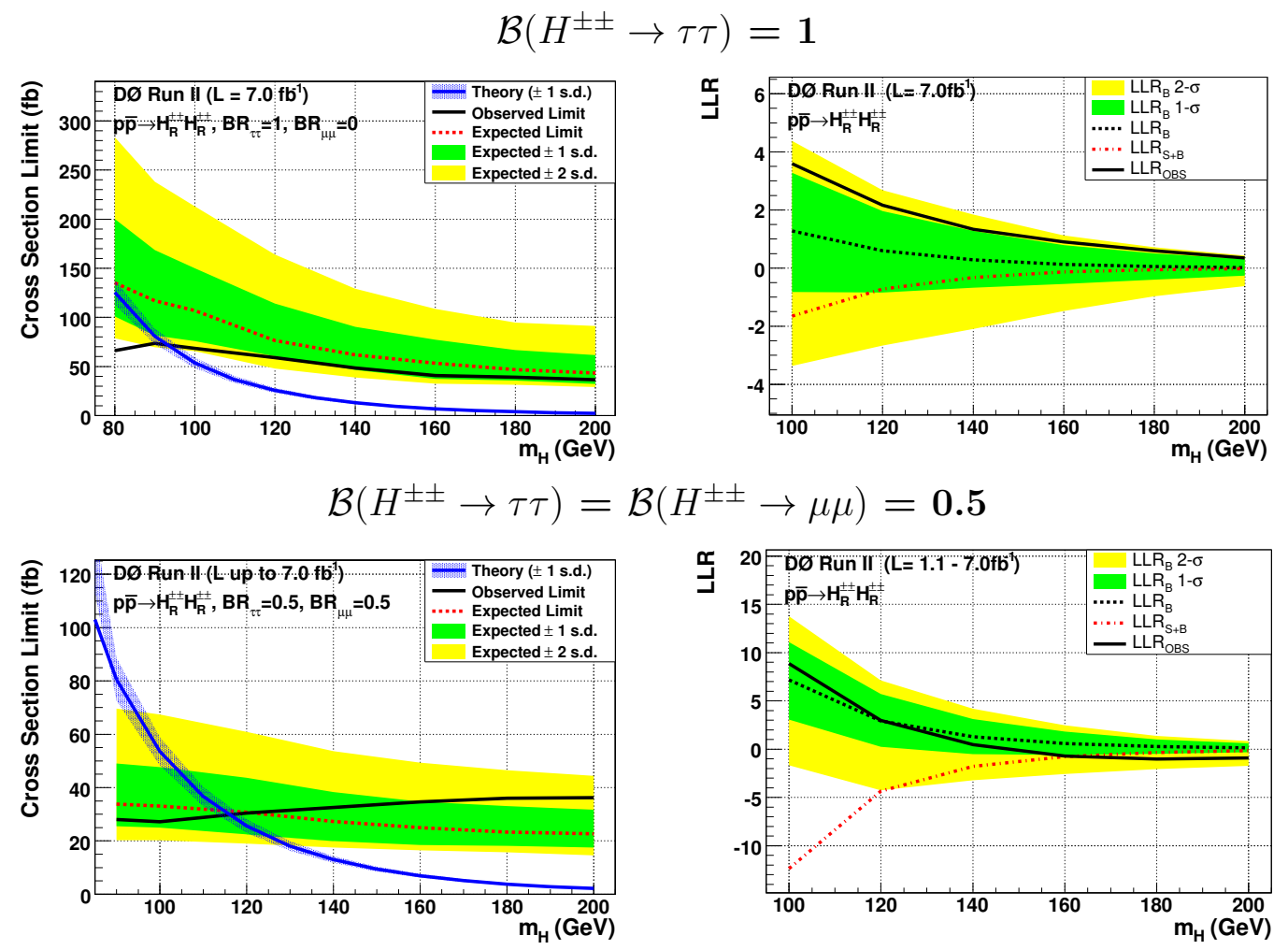

$$
\mathcal{B}\left(H^{ \pm \pm} \rightarrow \mu \mu\right)=\mathbf{1}
$$
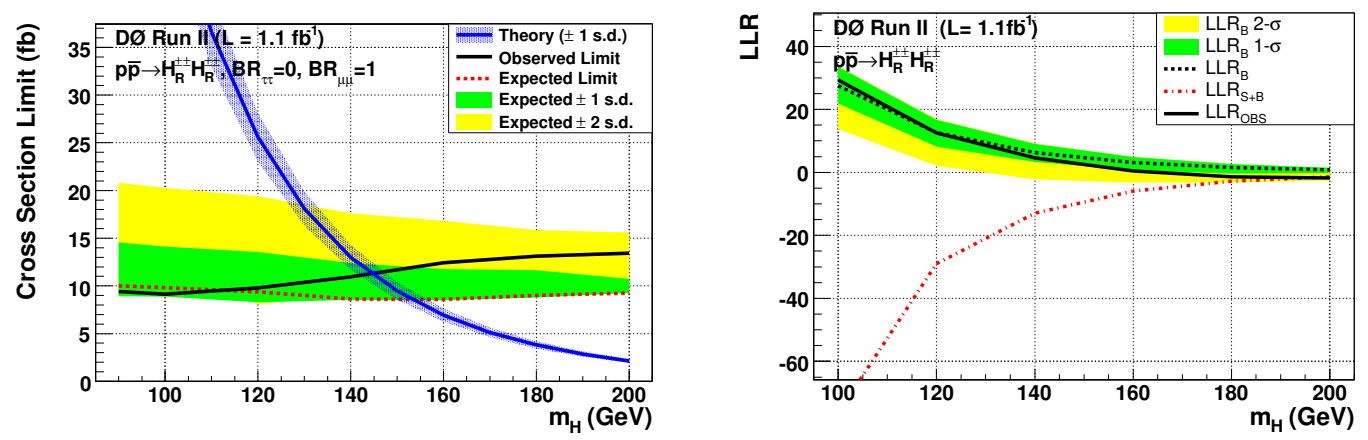

Figure 8.11: Expected and observed cross section limits, at 95\% C.L, and the corresponding LLR distributions for a right handed doubly charged Higgs boson, for benchmark points (1) (top), a specific case of benchmark point (2), $\mathcal{B}\left(H^{ \pm \pm} \rightarrow \tau \tau\right)$ $=\mathcal{B}\left(H^{ \pm \pm} \rightarrow \mu \mu\right)=0.5$ (middle), and benchmark point (3) (bottom). The observed and expected cross section limits are shown by the black and red lines, respectivaly. The yellow and green bands correspond to the $\pm 1 \sigma$ and $\pm 2 \sigma$ variation on the expected limits. The blue band in the limit plot shows the theoretical cross section and its uncertainty. 


\begin{tabular}{c|c|cccccc}
\multicolumn{1}{c}{$H_{\mathrm{L}}^{ \pm \pm}$boson cross section limits, $\left(M_{H^{ \pm \pm}},[\mathrm{GeV}]\right)$} \\
\hline Limits & $\mathcal{B}_{\tau \tau}[\%]$ & 100 & 120 & 140 & 160 & 180 & 200 \\
\hline$\sigma_{\text {exp }}[\mathrm{fb}]$ & 100 & 79.2 & 64.8 & 51.0 & 39.4 & 37.6 & 32.7 \\
$\sigma_{\text {obs }}[\mathrm{fb}]$ & & 49.0 & 47.1 & 38.0 & 30.8 & 32.3 & 28.5 \\
\hline$\sigma_{\text {exp }}[\mathrm{fb}]$ & 90 & 71.6 & 61.0 & 48.6 & 37.8 & 35.7 & 31.8 \\
$\sigma_{\text {obs }}[\mathrm{fb}]$ & & 43.0 & 45.7 & 39.7 & 36.7 & 36.6 & 32.6 \\
\hline$\sigma_{\text {exp }}[\mathrm{fb}]$ & 80 & 59.8 & 53.4 & 42.8 & 34.8 & 32.5 & 29.4 \\
$\sigma_{\text {obs }}[\mathrm{fb}]$ & & 37.1 & 43.7 & 42.8 & 39.4 & 39.6 & 35.8 \\
\hline$\sigma_{\text {exp }}[\mathrm{fb}]$ & 70 & 48.7 & 43.1 & 36.4 & 30.9 & 28.6 & 26.2 \\
$\sigma_{\text {obs }}[\mathrm{fb}]$ & & 31.8 & 37.5 & 39.7 & 38.1 & 39.1 & 36.2 \\
\hline$\sigma_{\text {exp }}[\mathrm{fb}]$ & 60 & 37.2 & 35.1 & 30.2 & 26.6 & 24.8 & 22.5 \\
$\sigma_{\text {obs }}[\mathrm{fb}]$ & & 26.4 & 33.3 & 34.4 & 34.9 & 35.7 & 33.7 \\
\hline$\sigma_{\text {exp }}[\mathrm{fb}]$ & 50 & 30.1 & 28.3 & 24.9 & 22.1 & 20.4 & 18.9 \\
$\sigma_{\text {obs }}[\mathrm{fb}]$ & & 22.3 & 28.2 & 29.8 & 30.3 & 31.0 & 30.1 \\
\hline$\sigma_{\text {exp }}[\mathrm{fb}]$ & 40 & 23.6 & 22.6 & 19.7 & 18.3 & 17.1 & 16.5 \\
$\sigma_{\text {obs }}[\mathrm{fb}]$ & & 18.5 & 23.2 & 25.0 & 26.0 & 27.1 & 26. \\
\hline$\sigma_{\text {exp }}[\mathrm{fb}]$ & 30 & 18.8 & 18.0 & 16.1 & 15.3 & 14.8 & 14.3 \\
$\sigma_{\text {obs }}[\mathrm{fb}]$ & & 16.2 & 19.0 & 20.8 & 21.9 & 22.9 & 22.5 \\
\hline$\sigma_{\text {exp }}[\mathrm{fb}]$ & 20 & 15.1 & 14.5 & 12.7 & 12.3 & 12.1 & 12.0 \\
$\sigma_{\text {obs }}[\mathrm{fb}]$ & & 13.5 & 15.4 & 16.6 & 17.8 & 18.8 & 18.3 \\
\hline$\sigma_{\text {exp }}[\mathrm{fb}]$ & 10 & 12.1 & 11.5 & 10.2 & 10.0 & 10.0 & 10.0 \\
$\sigma_{\text {obs }}[\mathrm{fb}]$ & & 10.8 & 12.1 & 13.8 & 14.7 & 15.4 & 15.0 \\
\hline$\sigma_{\text {exp }}[\mathrm{fb}]$ & 0 & 9.7 & 9.3 & 8.4 & 8.3 & 8.4 & 8.6 \\
$\sigma_{\text {obs }}[\mathrm{fb}]$ & & 9.0 & 9.6 & 10.3 & 11.8 & 12.1 & 12.0
\end{tabular}

Table 8.8: Expected and observed cross section limits at 95\% C.L., for $\mathcal{B}\left(H^{ \pm \pm} \rightarrow\right.$ $\tau \tau)+\mathcal{B}\left(H^{ \pm \pm} \rightarrow \mu \mu\right)=1$, covering benchmark points (1), (2) and (3), per $H_{\mathrm{L}}^{ \pm \pm}$ boson masses. $\mathcal{B}_{\tau \tau}$ is the percentage of of the $H_{\mathrm{L}}^{ \pm \pm}$boson decaying to tau leptons. 


\begin{tabular}{c|c|cccccc}
\multicolumn{1}{c}{$H_{\mathrm{R}}^{ \pm \pm}$boson cross section limits, $\left(M_{H^{ \pm \pm},}[\mathrm{GeV}]\right)$} \\
\hline Limits & $\mathcal{B}_{\tau \tau}[\%]$ & 100 & 120 & 140 & 160 & 180 & 200 \\
\hline$\sigma_{\text {exp }}[\mathrm{fb}]$ & 100 & 106.9 & 76.4 & 61.8 & 53.2 & 46.9 & 43.4 \\
$\sigma_{\text {obs }}[\mathrm{fb}]$ & & 68.4 & 58.9 & 48.4 & 40.8 & 39.1 & 36.6 \\
\hline$\sigma_{\text {exp }}[\mathrm{fb}]$ & 90 & 97.9 & 71.8 & 59.0 & 51.2 & 44.7 & 42.0 \\
$\sigma_{\text {obs }}[\mathrm{fb}]$ & & 60.9 & 57.4 & 51.8 & 48.2 & 45.6 & 44.7 \\
\hline$\sigma_{\text {exp }}[\mathrm{fb}]$ & 80 & 79.5 & 62.6 & 52.2 & 44.2 & 40.2 & 36.6 \\
$\sigma_{\text {obs }}[\mathrm{fb}]$ & & 52.6 & 52.8 & 52.1 & 50.9 & 49.3 & 48.6 \\
\hline$\sigma_{\text {exp }}[\mathrm{fb}]$ & 70 & 59.7 & 50.2 & 42.6 & 37.0 & 33.7 & 31.9 \\
$\sigma_{\text {obs }}[\mathrm{fb}]$ & & 40.2 & 44.3 & 46.5 & 46.9 & 46.7 & 46.3 \\
\hline$\sigma_{\text {exp }}[\mathrm{fb}]$ & 60 & 44.3 & 38.5 & 33.7 & 31.0 & 28.6 & 27.5 \\
$\sigma_{\text {obs }}[\mathrm{fb}]$ & & 33.5 & 36.9 & 39.5 & 41.3 & 41.7 & 42.1 \\
\hline$\sigma_{\text {exp }}[\mathrm{fb}]$ & 50 & 33.0 & 30.7 & 27.3 & 25.0 & 23.3 & 22.7 \\
$\sigma_{\text {obs }}[\mathrm{fb}]$ & & 27.2 & 30.4 & 32.6 & 34.6 & 36.0 & 36.3 \\
\hline$\sigma_{\text {exp }}[\mathrm{fb}]$ & 40 & 25.7 & 23.5 & 20.9 & 19.7 & 19.3 & 18.9 \\
$\sigma_{\text {obs }}[\mathrm{fb}]$ & & 21.4 & 24.7 & 26.9 & 28.6 & 30.1 & 30.7 \\
\hline$\sigma_{\text {exp }}[\mathrm{fb}]$ & 30 & 19.0 & 18.3 & 16.9 & 16.2 & 16.1 & 16.0 \\
$\sigma_{\text {obs }}[\mathrm{fb}]$ & & 17.0 & 19.3 & 21.6 & 23.5 & 25.1 & 25.6 \\
\hline$\sigma_{\text {exp }}[\mathrm{fb}]$ & 20 & 15.8 & 14.7 & 13.7 & 12.9 & 13.5 & 13.8 \\
$\sigma_{\text {obs }}[\mathrm{fb}]$ & & 14.6 & 15.7 & 17.3 & 19.1 & 20.4 & 20.7 \\
\hline$\sigma_{\text {exp }}[\mathrm{fb}]$ & 10 & 12.0 & 11.7 & 10.4 & 10.2 & 10.8 & 10.8 \\
$\sigma_{\text {obs }}[\mathrm{fb}]$ & & 11.0 & 12.2 & 14.3 & 15.5 & 16.5 & 16.9 \\
\hline$\sigma_{\text {exp }}[\mathrm{fb}]$ & 0 & 9.8 & 9.4 & 8.6 & 8.6 & 9.0 & 9.2 \\
$\sigma_{\text {obs }}[\mathrm{fb}]$ & & 9.1 & 9.8 & 10.9 & 12.4 & 13.1 & 13.4
\end{tabular}

Table 8.9: Expected and observed cross section limits at 95\% C.L., for $\mathcal{B}\left(H^{ \pm \pm} \rightarrow\right.$ $\tau \tau)+\mathcal{B}\left(H^{ \pm \pm} \rightarrow \mu \mu\right)=1$, covering benchmark points (1), (2) and (3), per $H_{\mathrm{R}}^{ \pm \pm}$ boson masses. $\mathcal{B}_{\tau \tau}$ is the percentage of of the $H^{ \pm \pm}$boson decaying to tau leptons. 

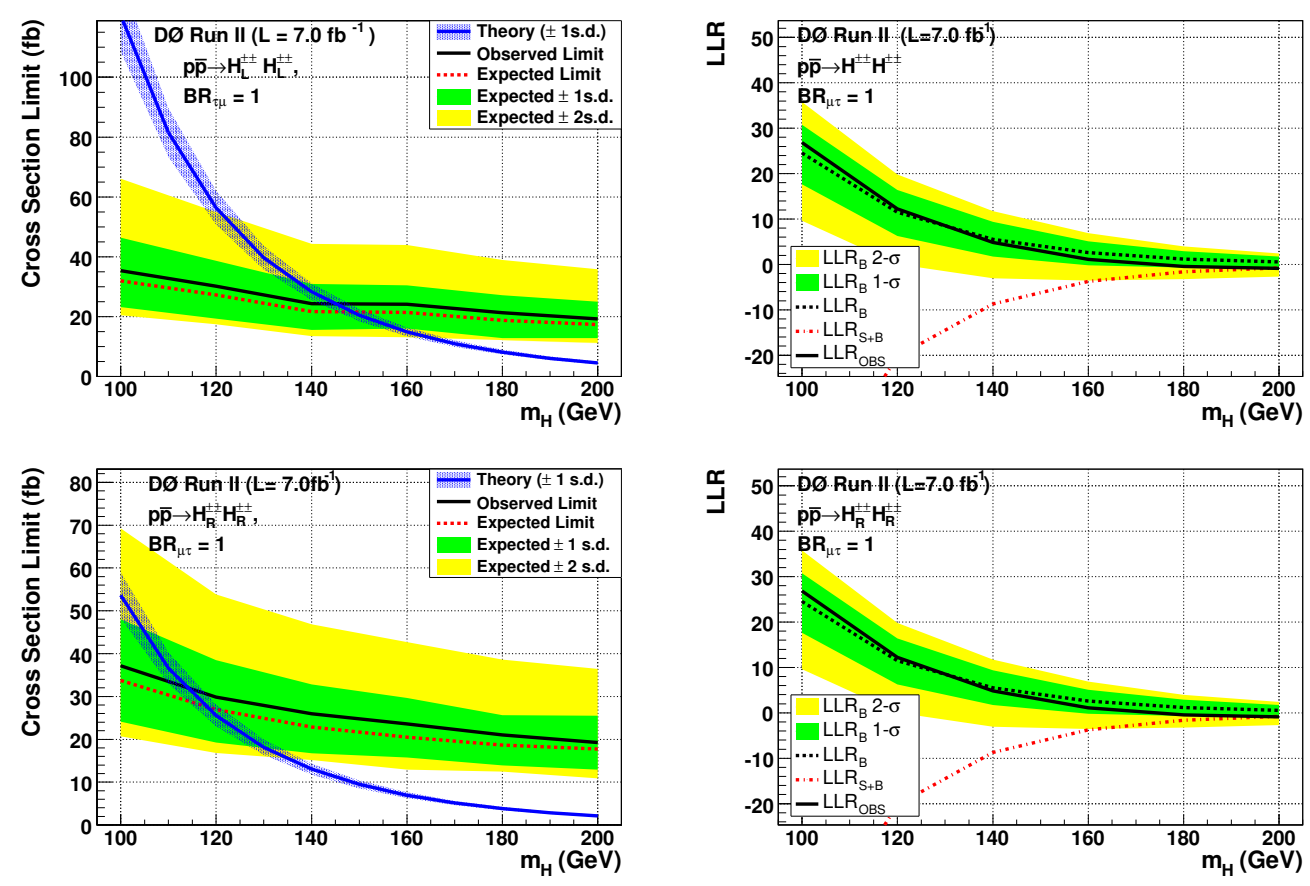

Figure 8.12: Expected and observed cross section limits at 95\% C.L and the corresponding LLR distributions for a left handed (top) and a right handed (bottom) doubly charged Higgs boson for benchmark point (4), with 100\% branching ratio to $\mu \tau$ pairs. The yellow and green bands correspond to the $\pm 1 \sigma$ and $\pm 2 \sigma$ variation on the expected limits, the blue band shows the theoretical cross section and its uncertainty.

\begin{tabular}{l|cccccc}
\hline$M_{H \pm \pm}[\mathrm{GeV}]$ & 100 & 120 & 140 & 160 & 180 & 200 \\
\hline \hline Left Handed & & & & & & \\
$\sigma_{\text {exp }}[\mathrm{fb}]$ & 31.6 & 27.2 & 21.7 & 21.7 & 18.6 & 17.3 \\
$\sigma_{\text {obs }}[\mathrm{fb}]$ & 35.7 & 30.9 & 25.3 & 24.8 & 21.8 & 20.2 \\
Right Handed & & & & & & \\
$\sigma_{\text {exp }}[\mathrm{fb}]$ & 33.8 & 26.3 & 22.8 & 20.8 & 18.5 & 17.4 \\
$\sigma_{\text {obs }}[\mathrm{fb}]$ & 37.9 & 30.6 & 26.5 & 24.1 & 21.7 & 20.5
\end{tabular}

Table 8.10: The expected and observed cross section limits at 95\% C.L for different $H^{ \pm \pm}$boson masses for the benchmark point (4), with $100 \%$ branching ratio to $\mu \tau$ pairs. 


\begin{tabular}{l|cccccc}
\hline$M_{H \pm \pm}[\mathrm{GeV}]$ & 100 & 120 & 140 & 160 & 180 & 200 \\
\hline \hline$\sigma_{\text {exp }}[\mathrm{fb}]$ & 54.5 & 43.5 & 35.7 & 31.5 & 28.5 & 27.2 \\
$\sigma_{\text {obs }}[\mathrm{fb}]$ & 35.6 & 33.7 & 30.0 & 27.6 & 25.5 & 24.7
\end{tabular}

Table 8.11: Expected and observed cross section limits at 95\% C.L., per $H^{ \pm \pm}$ boson masses for the benchmark point (5), for a $H^{ \pm \pm}$boson with equal branching ratios to $\mu \mu, \tau \tau$ and $\mu \tau$ lepton pairs.
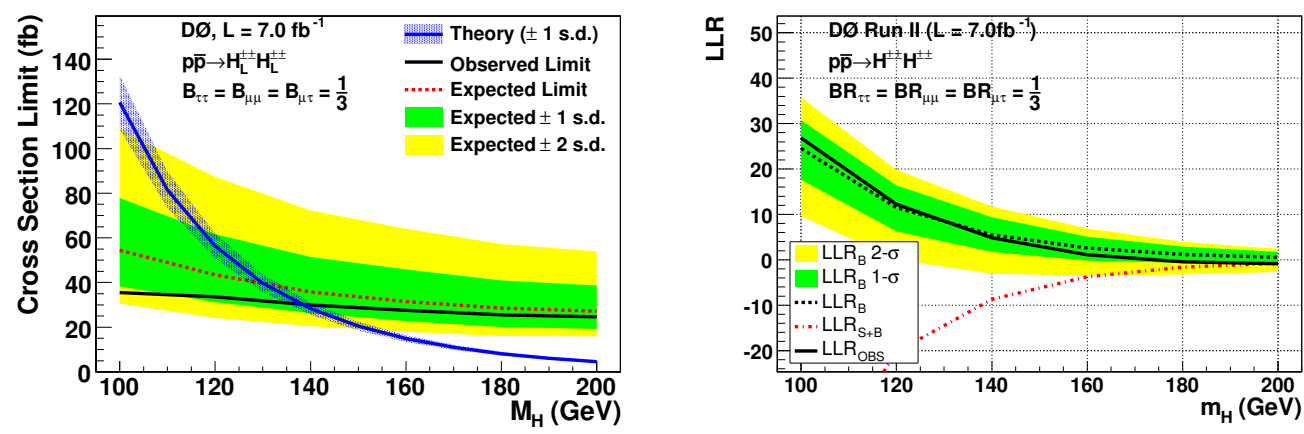

Figure 8.13: Expected and observed ross section limits for a left handed doubly charged Higgs boson,for benchmark point (5) with $\mathcal{B}\left(H^{++} \rightarrow \tau \tau\right)=\mathcal{B}\left(H^{++} \rightarrow\right.$ $\mu \mu)=\mathcal{B}\left(H^{++} \rightarrow \mu \tau\right)=\frac{1}{3}$ (left). Shown at $95 \%$ C.L (left) with the corresponding LLR plot (right). The yellow and green bands correspond to the $\pm 1 \sigma$ and $\pm 2 \sigma$ variation on the expected limits, the blue band shows the theoretical cross section and its uncertainty. 


\begin{tabular}{c|c|c|c|c|c}
\multirow{2}{*}{ BP } & $H^{ \pm \pm}$branching ratio & \multicolumn{2}{|c|}{$M\left(H_{L}^{ \pm \pm}\right)[\mathrm{GeV}]$} & \multicolumn{2}{|c}{$M\left(H_{R}^{ \pm \pm}\right)[\mathrm{GeV}]$} \\
\cline { 3 - 6 } & & $\sigma_{\text {exp }}[\mathrm{fb}]$ & $\sigma_{\text {obs }}[\mathrm{fb}]$ & $\sigma_{\exp }[\mathrm{fb}]$ & $\sigma_{o b s}[\mathrm{fb}]$ \\
\hline 1 & $\mathcal{B}_{\tau \tau}=1$ & 116 & 128 & NS & 94 \\
& $\mathcal{B}_{\tau \tau}=0.9, \mathcal{B}_{\mu \mu}=0.1$ & 118 & 128 & NS & 97 \\
& $\mathcal{B}_{\tau \tau}=0.8, \mathcal{B}_{\mu \mu}=0.2$ & 123 & 128 & NS & 101 \\
& $\mathcal{B}_{\tau \tau}=0.7, \mathcal{B}_{\mu \mu}=0.3$ & 130 & 131 & 98 & 108 \\
& $\mathcal{B}_{\tau \tau}=0.6, \mathcal{B}_{\mu \mu}=0.4$ & 138 & 135 & 107 & 112 \\
2 & $\mathcal{B}_{\tau \tau}=0.5, \mathcal{B}_{\mu \mu}=0.5$ & 146 & 139 & 115 & 117 \\
& $\mathcal{B}_{\tau \tau}=0.4, \mathcal{B}_{\mu \mu}=0.6$ & 154 & 144 & 124 & 122 \\
& $\mathcal{B}_{\tau \tau}=0.3, \mathcal{B}_{\mu \mu}=0.7$ & 160 & 149 & 132 & 128 \\
& $\mathcal{B}_{\tau \tau}=0.2, \mathcal{B}_{\mu \mu}=0.8$ & 167 & 156 & 139 & 133 \\
& $\mathcal{B}_{\tau \tau}=0.1, \mathcal{B}_{\mu \mu}=0.9$ & 174 & 161 & 148 & 138 \\
3 & $\mathcal{B}_{\mu \mu}=1$ & 180 & 168 & 154 & 145 \\
4 & $\mathcal{B}_{\tau \mu}=1$ & 149 & 144 & 119 & 113 \\
5 & Equal $\mathcal{B}$ & 130 & 138 & - & -
\end{tabular}

Table 8.12: Expected and observed lower mass limits for the 5 benchmark points studied. These are shown for both the left and the right handed doubly charged Higgs boson at $95 \%$ C.L. NS implies that no limit greater than $90 \mathrm{GeV}$ was set for the point. The branching ratio of the $H^{ \pm \pm}$per benchmark point is also shown. 


\section{Chapter 9}

\section{SM Higgs bosons in the $\tau \tau \mu+X$}

\section{final states}

In this section details are given for a search for the SM Higgs boson in $\tau \tau \mu+X$ final states. This search is performed using Run II data using a total integrated luminosity of $8.6 \mathrm{fb}^{-1}$ and requiring at least one muon and two hadronically decaying tau leptons in the final state. The " $X$ " implies that there is no explicit veto on all extra leptons and energy in the event.

At the Tevatron the primary SM Higgs boson production mechanism is gluongluon fusion, $g g \rightarrow H$. The associated production of a Higgs boson with a $W$ or $Z$ boson also contributes, $q \bar{q} \rightarrow W \rightarrow W H$ and $q \bar{q} \rightarrow Z \rightarrow Z H$, as shown in Figure 9.1, and there is a small contribution from vector boson fusion, $q \bar{q} \rightarrow q \bar{q} H+X$. This analysis is expected to be mainly sensitive to a Higgs boson produced through associated production due to the requirement on a third lepton. The analysis will be sensitive to SM Higgs bosons decaying as $H \rightarrow \tau \tau$ at masses below $130 \mathrm{GeV}$, and to $H \rightarrow W W$ and $H \rightarrow Z Z$ decays at higher masses. This is the first time this final state has been studied at $\mathrm{D} \varnothing$, and it has the advantage of being sensitive across the whole mass range accessible at the Tevatron. In addition it explores a fermionic decay channel where no evidence for a SM Higgs 
boson like excess, as was observed by the CMS and ATLAS experiments, has been explicitly seen.
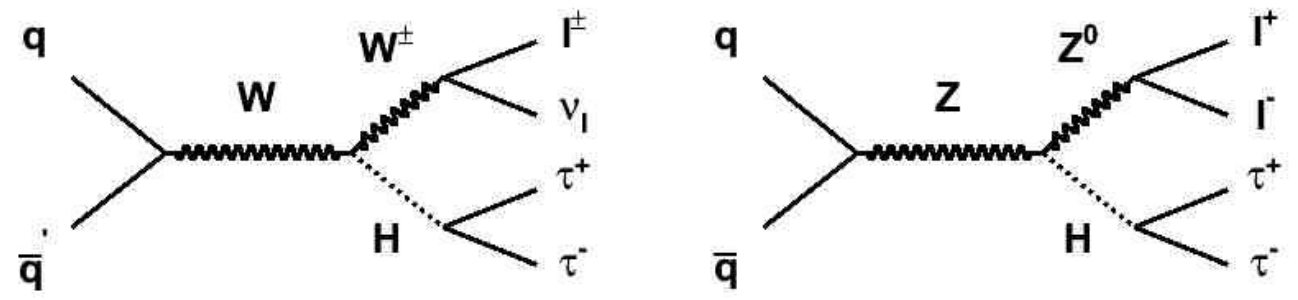

Figure 9.1: A Feynman diagram showing the associated production of a SM Higgs with a $W$ or $Z$ boson, where the Higgs boson decays into tau leptons.

This analysis is complementary to other DØ analyses looking for a SM Higgs boson in $\mu \mu e$ and $e e \mu$ three lepton final states. This subset of analyses are referred to as "trilepton" analyses. The limits determined in this analysis are used in the production of the combined $\mathrm{D} \varnothing$ and Tevatron limits on the cross section of a SM Higgs boson [1] [2].

\subsection{Data samples}

The analysis presented here is conducted using the Run IIb 1-4 data, collected between June 2006 and September 2011 and corresponding to physics Runs taken within the range 221698 to 275727 . The MuTau skims, as described in Section 7.1, are used for all data taking epochs. The data quality requirements as described in Section 7.1.2 are applied. 


\subsection{Monte Carlo samples}

The generated MC samples for both the predicted SM background events and the predicted signal for a SM Higgs boson, will be covered in this section.

\subsubsection{Background Monte Carlo samples}

The background MC samples described in Section 7.3 and listed in Tables 7.3 and 7.4 are used, normalized to the used integrated luminosity $\left(L=8.6 \mathrm{fb}^{-1}\right)$ and reweighted to the SingleMuonOr trigger. The k-factors given in Table 7.5 are applied, along with the MC corrections as described in Section 7.4.

\subsubsection{Signal Monte Carlo samples}

MC samples are generated for the production of a SM Higgs boson in the following modes:

- A SM Higgs boson produced in association with a $W$ or $Z$ boson, $W H$ and $Z H$.

- A SM Higgs boson produced by vector boson fusion, VBF.

- A SM Higgs boson produced through gluon-gluon fusion, decaying to two bosons, $H \rightarrow Z Z$ or $H \rightarrow W W$.

All decay modes that result in a three-lepton final state are allowed for VBF and associated production modes. For a SM Higgs boson these are $H \rightarrow W W$, $H \rightarrow Z Z, H \rightarrow \gamma \gamma, H \rightarrow e e, H \rightarrow \mu \mu, H \rightarrow \tau \tau$, and $H \rightarrow \gamma Z$. All Higgs boson production modes are calculated using the MSTW 2008 PDF sets. The $W H$ and $Z H$ production cross sections are calculated at NNLO [120], the VBF cross sections are calculated at NNLO in QCD, the gluon-gluon fusion cross section is calculated at NNLO and next-to-next to leading log (NNLL), which has a 
smaller scale dependence than the NNLO calculation [121]. The product of the cross sections and branching ratios are listed in Table 9.2, per generated mass point. The signal samples are generated in the mass range of $100-200 \mathrm{GeV}$ at $5 \mathrm{GeV}$ intervals, using the PYTHIA event generator version 6.323 [62]. The tau lepton decays are modeled by the TAUOLA package [78].

\subsection{Inclusive trigger approach}

This analysis uses what is known as an inclusive trigger approach. This means it does not require selected events to pass any one specific trigger but rather any of the DØtriggers. This method was developed to increase the selection efficiency, by allowing all events that meet the selection criteria be used regardless of if they passed a specific trigger. As the efficiency for selecting data events with this inclusive trigger approach is not known, it has to be determined from data in order to derive a correction to apply to the simulated MC events. This is done by determining the increase in events selected with the inclusive trigger, i.e with no trigger requirement, as compared to those selected by the SingleMuonOr trigger. The SingleMuonOr trigger (Section 7.2) has a well known efficiency [56], and a large fraction of the events $(\approx 70 \%)$ that are selected by the inclusive trigger pass the SingleMuonOr trigger.

Before determining the inclusive trigger efficiency, it must be confirmed that data and MC distributions agree, when using the SignalMuonOr trigger. Figure 9.3 (top) shows the distribution at final selection with the SignalMuonOr trigger applied to data and the corresponding efficiency applied to MC events. The corresponding numbers of predicted SM and observed data events, are listed in Table 9.1.

In determining the inclusive trigger efficiency a sample is selected that is similar to the final selection, so the correction will be applicable to that sample, while 


\begin{tabular}{l|ccccccc} 
& \multicolumn{7}{|c}{$\sigma \times \mathrm{BR}[\mathrm{pb}]$} \\
\hline Mass [GeV] & $W H$ & $Z H$ & \multicolumn{7}{c}{$H \rightarrow Z Z \rightarrow$} & & $H \rightarrow W W$ & VBF \\
& & & $\ell \ell \ell \ell j$ & $\ell \ell \nu \nu$ & & \\
\hline 100 & 0.0296 & 0.0172 & - & - & - & - & - \\
105 & 0.0259 & 0.0152 & - & - & - & - & - \\
110 & 0.0231 & 0.0137 & - & - & - & - & - \\
115 & 0.0209 & 0.0120 & 0.00011 & 0.00150 & 0.00043 & 0.0111 & 0.00072 \\
120 & 0.0191 & 0.0110 & 0.00018 & 0.00242 & 0.00069 & 0.0161 & 0.00109 \\
125 & 0.0182 & 0.0107 & 0.00026 & 0.00358 & 0.00102 & 0.0215 & 0.00152 \\
130 & 0.0179 & 0.0108 & 0.00036 & 0.00478 & 0.00137 & 0.0270 & 0.00199 \\
135 & 0.0174 & 0.0102 & 0.00042 & 0.00584 & 0.00167 & 0.0318 & 0.00243 \\
140 & 0.0164 & 0.0098 & 0.00047 & 0.00655 & 0.00187 & 0.0355 & 0.00282 \\
145 & 0.0159 & 0.0096 & 0.00049 & 0.00675 & 0.00193 & 0.0380 & 0.00313 \\
150 & 0.0149 & 0.0091 & 0.00046 & 0.00630 & 0.00180 & 0.0396 & 0.00336 \\
155 & 0.0140 & 0.0084 & 0.00036 & 0.00503 & 0.00144 & 0.0405 & 0.00354 \\
160 & 0.0127 & 0.0077 & 0.00018 & 0.00254 & 0.00073 & 0.0413 & 0.00376 \\
165 & 0.0121 & 0.0069 & 0.00009 & 0.00120 & 0.00034 & 0.0387 & 0.00369 \\
170 & 0.0106 & 0.0061 & 0.00008 & 0.00115 & 0.00033 & 0.0349 & 0.00345 \\
175 & 0.0092 & 0.0056 & 0.00010 & 0.00141 & 0.00040 & 0.0311 & 0.00318 \\
180 & 0.0081 & 0.0051 & 0.00017 & 0.00237 & 0.00068 & 0.0273 & 0.00288 \\
185 & 0.0075 & 0.0046 & 0.00039 & 0.00534 & 0.00153 & 0.0223 & 0.00242 \\
190 & 0.0066 & 0.0042 & 0.00049 & 0.00673 & 0.00193 & 0.0188 & 0.00210 \\
195 & 0.0056 & 0.0039 & 0.00051 & 0.00699 & 0.00200 & 0.0165 & 0.00188 \\
200 & 0.0052 & 0.0035 & 0.00049 & 0.00684 & 0.00196 & 0.0147 & 0.00171
\end{tabular}

Figure 9.2: The product of the cross section and branching ratio in pb for the signal MC samples generated, given for the range of SM Higgs masses studied, 100 to $200 \mathrm{GeV}$. For a Higgs boson produced through gluon-gluon fusion and decaying to $Z$ bosons, the product of the cross section and BR is given separately, for the decay into four leptons, $H \rightarrow Z Z \rightarrow \ell \ell \ell \ell$, the decay into two leptons and two neutrinos $H \rightarrow Z Z \rightarrow \ell \ell \nu \nu$, and the decay into two leptons and two jets, $H \rightarrow Z Z \rightarrow \ell \ell j j$. 

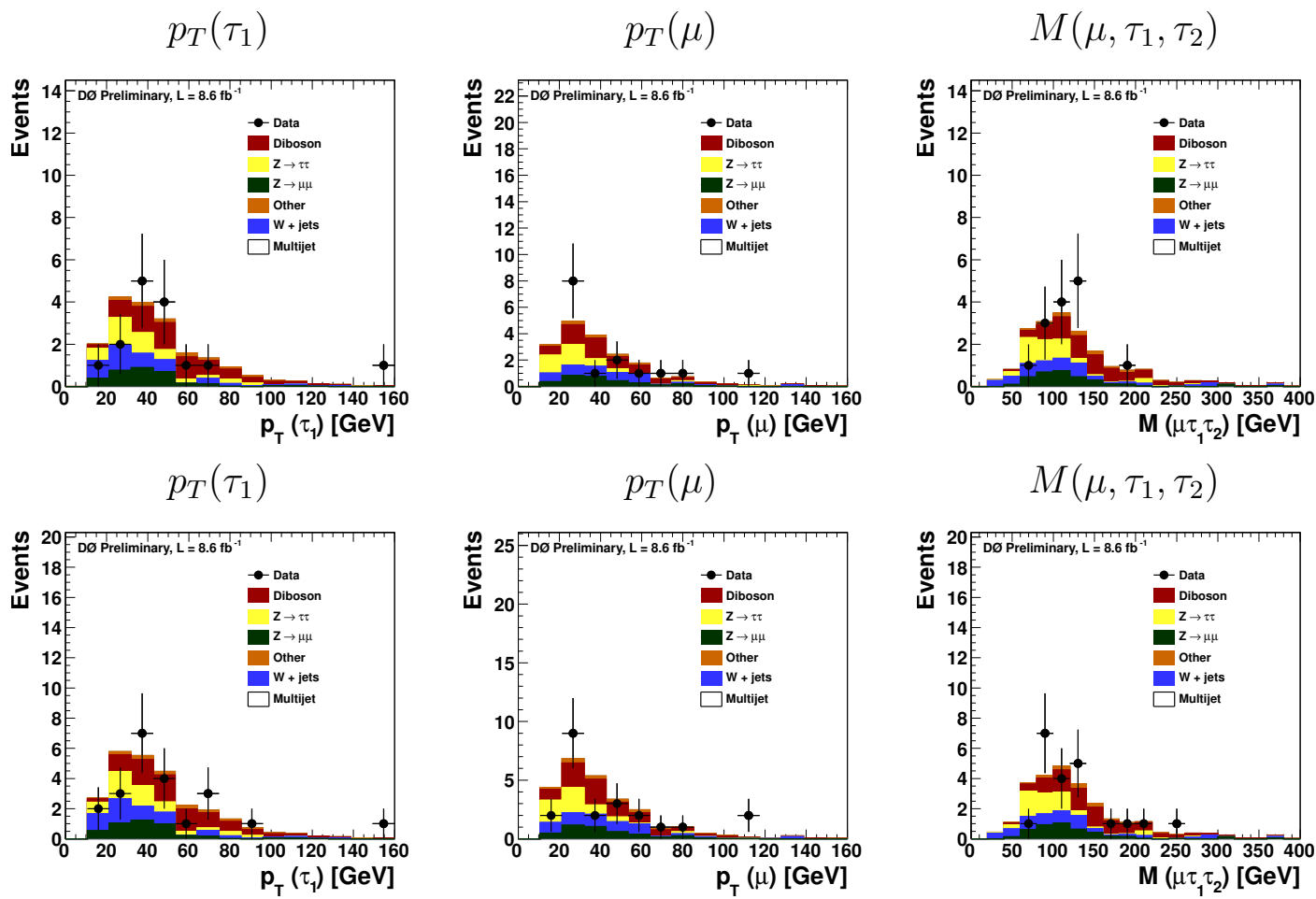

Figure 9.3: Data distributions at final selection using the SingleMuonOr trigger (top) and inclusive trigger (bottom) compared to the sum of the expected background from MC simulations with their respective corrections applied. The 'Other' background sample contains the contributions from $Z \rightarrow e e$ and $t \bar{t}$ events.

retaining enough statistics to make an accurate determination of the efficiency. The selection requirements are taken to be the same as the final selection (Section 9.6.4), with the $N N_{\tau}$ requirement relaxed to $N N_{\tau}>0.05$ for all tau lepton types. This $N N_{\tau}$ requirement removes the dominant peak of multijet events at zero while retaining enough statistics to perform an accurate determination of the efficiency. To remove a large portion of the remaining multijet events, a "triangle cut" [122] on the mass of the $W$ boson is applied, $M_{W}>\left(0-0.5 E_{\mathrm{T}}\right) \mathrm{GeV}$, where the $W$ boson mass is calculated as $M_{W}=\sqrt{2 E^{\mu} E^{\nu}\left(1-\cos \left(\Delta \phi_{\mu \nu}\right)\right)}$ in $\mathrm{GeV}$, and $\Delta \phi_{\mu \nu}$ is the angle between the muon and the $\mathbb{E}_{\mathrm{T}}$ direction in the $r \phi$ plane. This cut has been shown to remove the multijet dominated region [109].

To compute the inclusive trigger efficiency, the following method is used: 


\begin{tabular}{l|c|c} 
& SingleMuonOr trigger & Inclusive trigger \\
\hline \hline Data & 15 & 22 \\
$Z / \gamma^{*} \rightarrow \tau \tau$ & $4.03 \pm 0.51$ & $5.56 \pm 0.70$ \\
$Z / \gamma^{*} \rightarrow \mu \mu$ & $3.15 \pm 0.40$ & $4.42 \pm 0.56$ \\
$Z / \gamma^{*} \rightarrow \mu \mu$ & $0.22 \pm 0.03$ & $0.31 \pm 0.04$ \\
$t \bar{t}$ & $1.01 \pm 0.15$ & $1.43 \pm 0.21$ \\
Diboson & $6.45 \pm 0.82$ & $9.01 \pm 1.15$ \\
$W+$ jet & $3.85 \pm 0.48$ & $5.38 \pm 0.68$ \\
Multijet & $0.00 \pm 1.47$ & $0.00 \pm 0.47$ \\
Total Bkg & $18.72 \pm 3.86$ & $27.22 \pm 3.81$
\end{tabular}

Table 9.1: Predictions from the MC simulations compared to the data for the SingleMuonOR and inclusive trigger approaches. Systematic uncertainties on the MC and multijet are given. The contribution from statistical uncertainties is taken to be negligible and not included.

1. The ratio of the number of data events that pass the SingleMuonOr trigger to the number of data events that pass the inclusive trigger requirement, $R_{\text {incl }}$, is determined from Equation 9.1,

$$
R_{\text {incl }}=\frac{N_{\text {data }}^{\text {incl }}}{N_{\text {data }}^{\text {SingleMuonOr }}},
$$

where $N_{\text {data }}^{\text {incl }}$ is the number of data events that pass the inclusive trigger requirement and $N_{\text {data }}^{\text {SingleMuon } O r}$ is the number of data events that pass the SingleMuonOr trigger requirement. $R_{\text {incl }}$ is measured as a function of various kinematic variables and it is found to be dependent on the $p_{T}$ of the highest $p_{T}$ tau lepton, $p_{T}^{\tau_{1}}[109]$. Therefore, $R_{\text {incl }}$ is measured in six bins of $p_{T}^{\tau_{1}}$.

2. The ratio $R_{\text {incl }}$ is then parameterized in $p_{T}^{\tau_{1}}$ using the following function,

$$
f_{R_{\text {incl }}}\left(p_{T}^{\tau_{1}}\right)=a+b+e^{\left(p_{T}^{\tau_{1}}-c / d\right)}
$$

where $a, b, c$, and $d$ are parameters determined in the fitting procedure. The 


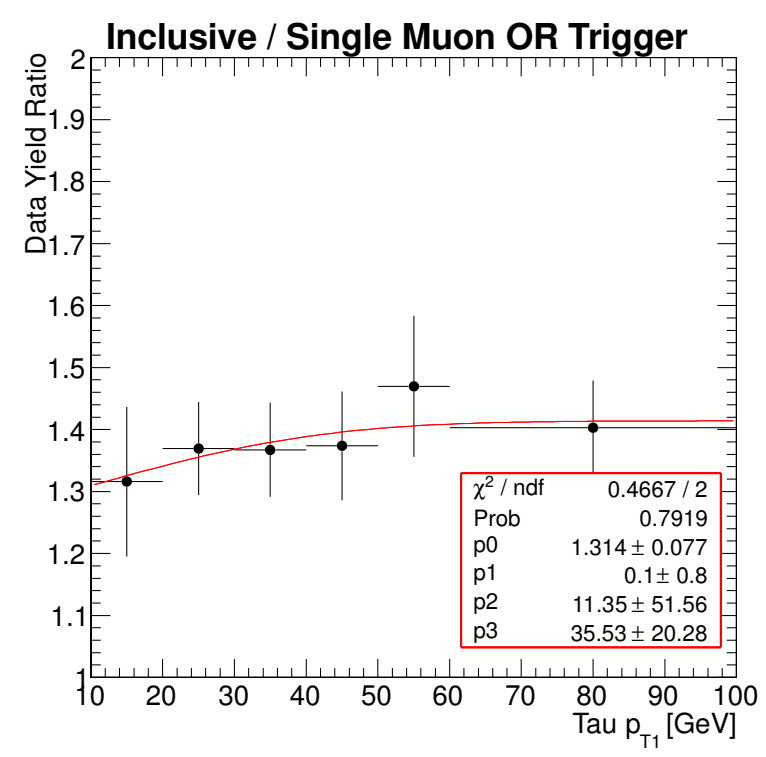

Figure 9.4: The ratio of the number of data events that pass the inclusive trigger to the number of data events that pass the SingleMuonOR, as a function of the $p_{T}$ of the highest $p_{T} \tau$ lepton. The fitted distribution, as given in Equation 9.2, is shown in red.

measured distribution of $R_{\text {incl }}$ in bins of $p_{T}^{\tau_{1}}$ is shown in Figure 9.4, along with the fitted function, $f_{R_{\text {incl }}}\left(p_{T}^{\tau_{1}}\right)$. The inclusive trigger method gives a gain in efficiency on the order of $40 \%$ as compared to the SingleMuonOr trigger. The uncertainty shown on $R_{\text {incl }}$ is the statistical uncertainty determined per $p_{T}^{\tau_{1}}$ bin from the number of events in the $N_{\text {data }}^{\text {incl }}$ and $N_{\text {data }}^{\text {SingleMuonOr }}$ data samples.

3. The trigger efficiency for the inclusive trigger, $\epsilon_{\text {trig }}$, can then been calculated from

$$
\epsilon_{\text {incl }}\left(p_{T}^{\tau_{1}}\right)=\epsilon_{s m O R} \times f_{R_{\text {incl }}}\left(p_{T}^{\tau_{1}}\right)
$$

where $\epsilon_{s m O R}$ is the SingleMuonOr trigger efficiency.

Applying the determined inclusive trigger efficiency correction to the MC 
samples and comparing to data events selected using the inclusive trigger method, good agreement is seen, as shown in Figure 9.3 (bottom). The corresponding number of events, for both the observed data and predicted MC background events, are listed in Table 9.1.

\subsection{W+jets normalization}

The $W+$ jets events are not well modeled by the MC [109]. Therefore the $W+$ jets normalization as described in Section 7.7 is applied. The normalization is applied separately per tau lepton type and for when the electric charge of the two tau leptons is matching and opposite. All $W+$ jets distributions used in this chapter will include this normalization, unless explicitly stated. The number of predicted $W+$ jet MC events is determined to be $627 \pm 169$ events at pre-selection and 5.36 \pm 0.16 at final selection, where the uncertainty given is the uncertainty due to the normalization procedure.

\subsection{Instrumental background estimation}

As is described in Section 7.8, heavy flavour multijet events are expected to contribute to the background events in this analysis. This background source is difficult to simulate with MC generators and is therefore determined directly from data. Two methods to estimate this background contribution are used, one based on the $N N_{\tau}$ requirement of the two tau leptons as described in Section 7.8.1. A second method acts as a cross check and is based on the absolute sum of the electric charges of the three reconstructed leptons and on the $N N_{\tau}$ of the highest $p_{T}$ tau lepton. This method is described in Section 7.8.2.

The multijet background is determined for the final selections sample as described in Section 8.5.1, hence the selection criteria for the TTNN and SR samples 
are required to match the selection criteria at final selection. The multijet contribution is determined independently for each of the three tau lepton types and for a sample with all tau lepton types combined.

The total predicted multijet contribution determined using instrumental background Method 1 is $2.25 \pm 1.35$ events when calculated per tau lepton type and $0.00 \pm 0.47$ events when calculated for all the tau leptons types combined. For the cross check method the total predicted multijet contribution is $0.55 \pm 1.88$ events per tau lepton type, and $0.00 \pm 0.39$ events events for all the tau leptons types combined. The values are listed in Table 9.2 with their uncertainty. The uncertainty is determined from the data statistics corresponding to the methods used, as the MC statistical uncertainties are small enough to be neglected.

\begin{tabular}{|c|c|}
\hline \multicolumn{2}{|c|}{ Instrumental background Method 1} \\
\hline Tau lepton Type & Multijet \& $W+$ jet \\
\hline Type-1 & $0.65 \pm 0.72$ \\
\hline Type-2 & $1.60 \pm 1.11$ \\
\hline Type-3 & $0.00 \pm 0.24$ \\
\hline Total & $2.25 \pm 1.35$ \\
\hline All Types & $0.00 \pm 0.47$ \\
\hline \multicolumn{2}{|c|}{ Instrumental background Method } \\
\hline Type-1 & $0.00 \pm 0.39$ \\
\hline Type-2 & $0.52 \pm 1.67$ \\
\hline Type-3 & $0.03 \pm 0.74$ \\
\hline Total & $0.55 \pm 1.88$ \\
\hline All Types & $0.00 \pm 0.39$ \\
\hline
\end{tabular}

Table 9.2: The multijet contribution shown both for instrumental background Method 1 and for instrumental background Method 2. It is given per tau lepton type, and with all tau lepton types combined, "All Types", along with the associated statistical uncertainty from the estimation method.

The contribution from the different methods are consistent with zero and with each other, except for instrumental background Method 1 determined per tau lepton type, which disagrees at the one standard deviation level. When determining 
the contribution per tau lepton type, the small statistics in the normalization and shape regions yields large $(>100 \%)$ errors. This implies that this separation into tau lepton types could lead to large unrepresentative fluctuations in the prediction. Therefore the multijet contribution used in the analysis is as determined from instrumental background Method 1 for all tau leptons types combined. The uncertainty is taken as the largest uncertainty that was determined by the two methods when determining the multijet contribution for all tau lepton types combined. The determined multijet contribution at final selection has been determined to be an upper limit of 0.47 events. This value is less than $2 \%$ of the total background contribution at final selection and is therefore considered negligible. Therefore no multijet contribution will be considered in this analysis at final selection.

The multijet events as shown at pre-selection level can be considered to be purely illustrative. The two multijet estimation methods are designed to be used with a $N N_{\tau}$ requirement, which is not applied until final selection. Adapting these methods to the pre-selection sample leads to a large correlation between the sample used for determining the shape of the multijet events and the sample for which the estimation is being applied to. This correlation between samples means that at pre-selection the multijet estimation will closely follow the data to MC event difference.

\subsection{Selection requirements}

The selection requirements used in this analysis at both pre-selection and final selection will be listed in this section, along with their motivations. 


\subsubsection{Pre-selection requirements}

At pre-selection this analysis applies the following requirements, in addition to those discussed in Section 7.5. The quality criteria on the muon are required to be; muon quality "MediumNseg3", track quality "TrackMedium", and isolation quality "TopScaledMedium", as defined in Section 4.3.1. The two tau leptons and the muon are required to be isolated, $\Delta R\left(\ell_{i}, \ell_{j}\right)>0.5$, for all lepton pair combinations, $\tau_{1} \tau_{2}, \tau_{1} \mu$, and $\tau_{2} \mu$.

\subsubsection{Muon and electron trilepton veto}

In order to ensure orthogonality with the other D $\varnothing$ trilepton analysis [123] which studied $\mu \mu e$ and $e e \mu$ lepton final states, events which satisfy the selection criteria of this analysis are removed from the selection. This ensures that for combined SM Higgs boson cross section limits there will be no double counting of Higgs boson events. The selection of electrons and muons used in the $\mu \mu e$ and $е e \mu$ trilepton analysis are as follows [123]:

- For the electrons: Point05 in CC, and Point1 in EC, as defined in Section 4.3.3.

- For the muons: Muon system quality: "Loose", track quality: "TrackNewMedium", Isolation quality: "TopScaledLoose", as defined in Ref. [55].

It is also required that all three leptons are isolated, $\Delta R\left(\ell_{i}, \ell_{j}\right)>0.3$ between all lepton pairs, and that the leptons fulfill the following requirements on their $p_{T}: p_{T}\left(\ell_{1}\right)>15 \mathrm{GeV}, p_{T}\left(\ell_{2}\right)>15 \mathrm{GeV}$, and $p_{T}\left(\ell_{3}\right)>10 \mathrm{GeV}$, where $\ell_{i}$ are ordered in the $p_{T}$ of the electron or the muon. As this analysis uses different skimming criteria (see Section 7.1) to the $\mu \mu e$ and $e e \mu$ trilepton analysis, it is not possible to apply exactly the same muon quality. At skimming level stricter constraints are placed on the muons used in this analysis then are placed on the muons for the $\mu \mu e$ 
and $e e \mu$ trilepton analysis. Therefore, all muons in this analysis will be a subset of the muons selected in the $\mu \mu e$ and $e e \mu$ trilepton analysis. Though electrons are not specifically used in this analysis, information on electrons matching these criteria are stored so that the relevant events can be removed. All events with two or more electrons or two or more muons matching the criteria listed above are removed from the analysis sample. The effect of this veto on the event yields for the predicted signal and background events is to reduce the predicted signal by $\approx 20 \%$ and the predicted background by $\approx 30 \%[109]$.

\subsubsection{Distributions at pre-selection}

The distributions of the predicted MC events compared to the observed data events at pre-selection level, as described in Section 9.6.1, are shown in Figures 9.5 and 9.6 for all tau lepton types combined, while the distributions for the respective tau lepton types are shown in Figure 9.7. The type of the highest $p_{T}$ tau lepton is used in classifying events. The corresponding number of data and predicted MC events, per tau lepton type and for all tau lepton types combined, are listed in Table 9.3. Samples that have only a small contribution at final selection are combined, these are the $Z / \gamma^{*} \rightarrow e e$ and $t \bar{t}$ samples. The signal distributions for a SM Higgs boson produced in associated with a $Z$ or $W$ boson, for a $\mathrm{SH}$ Higgs boson mass of $125 \mathrm{GeV}$ are also shown superimposed on these distributions, multiplied by a factor of 1000 . The same selection of distributions is plotted as in Section 8.4.2.

\subsubsection{Final selection requirements}

As good agreement is seen at pre-selection, additional requirements can be implemented beyond those specified in Section 9.6.1. These are designed to further reduce the background from $Z / \gamma^{*} \rightarrow \tau \tau, Z / \gamma^{*} \rightarrow \mu \mu, W+$ jets, and multijet events, 

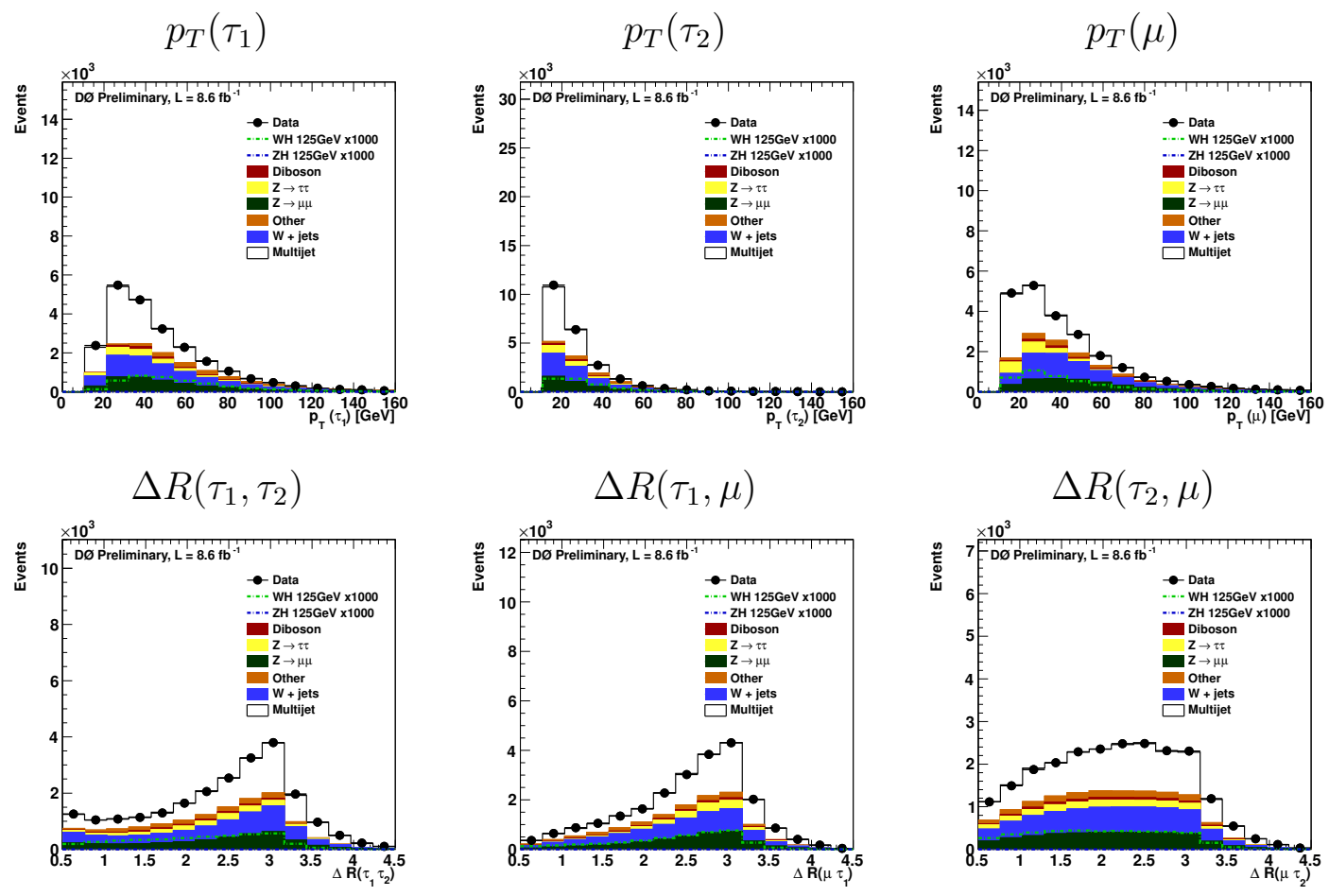

$N_{\mu}$

$N_{\tau}$
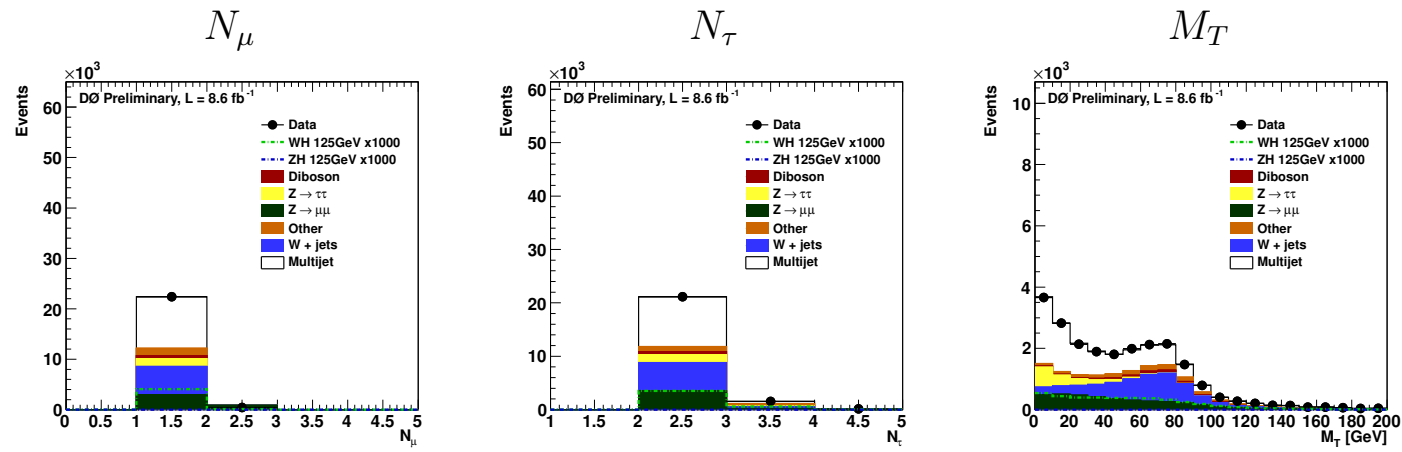

Figure 9.5: Data distributions at pre-selection compared to the sum of the expected backgrounds from $\mathrm{MC}$ simulations and multijet background methods. The MC distribution of the associated production of a SM Higgs boson produced with a $Z$ and $W$ boson and a mass of $125 \mathrm{GeV}$, multiplied by a factor of 1000 , are superimposed. The 'Other' background sample contains the contributions from $Z \rightarrow e e$ and $t \bar{t}$ events. 

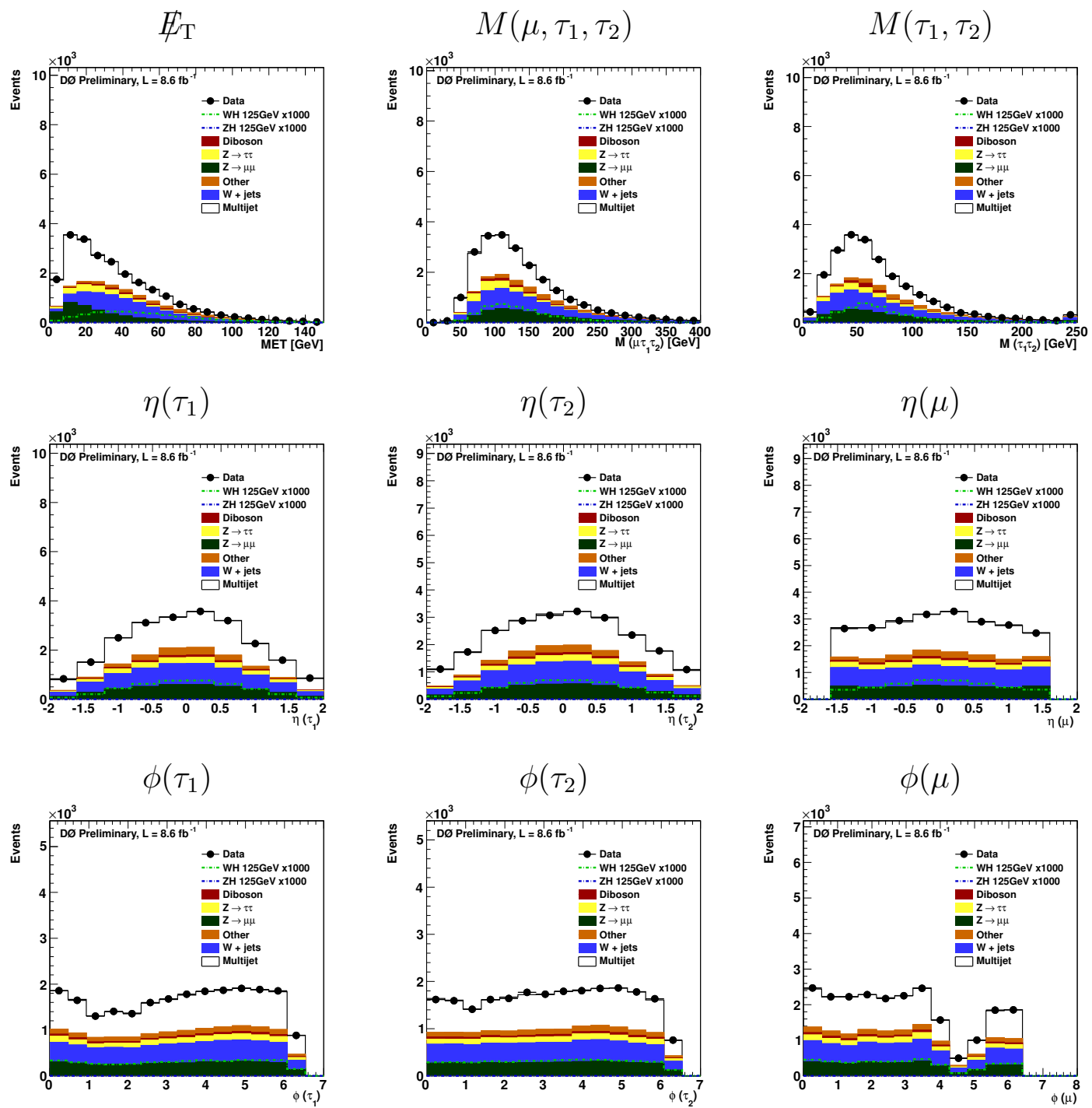

Figure 9.6: Data distributions at pre-selection compared to the sum of the expected backgrounds from MC simulations and multijet background methods. The MC distribution of the associated production of a SM Higgs boson produced with a $Z$ and $W$ boson and a mass of $125 \mathrm{GeV}$, multiplied by a factor of 1000 , are superimposed. The 'Other' background sample contains the contributions from $Z \rightarrow e e$ and $t \bar{t}$ events. 


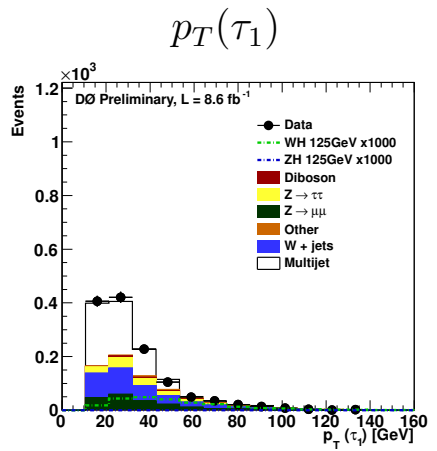

Tau lepton Type-1
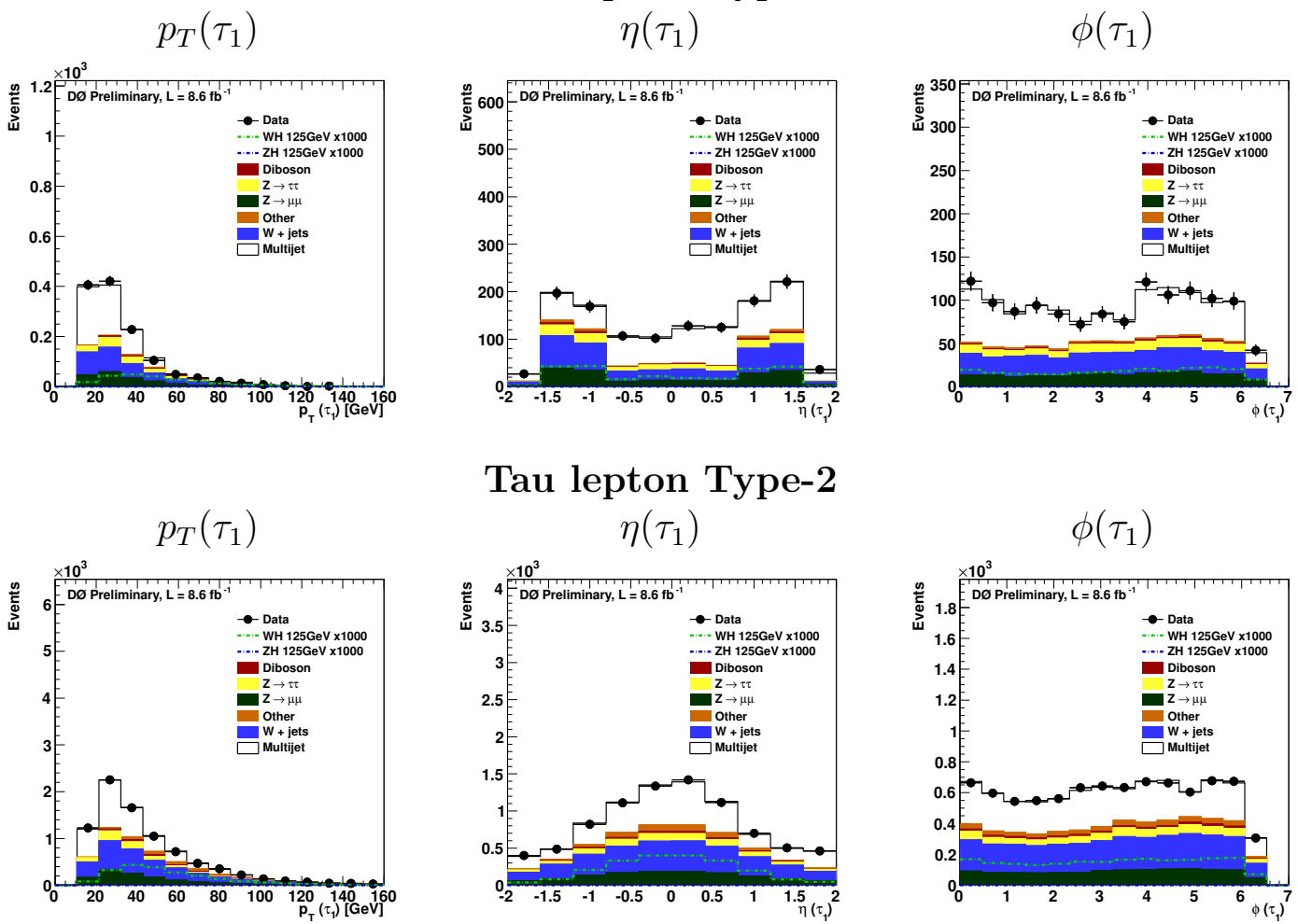

Tau lepton Type-2
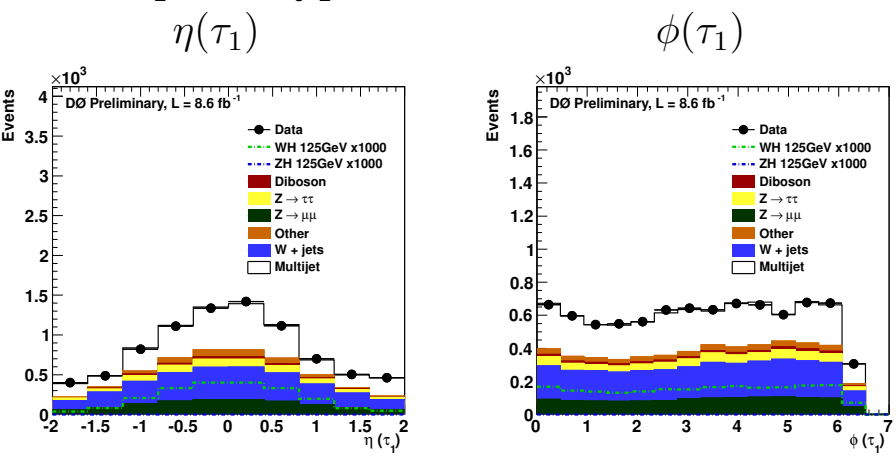

Tau lepton Type-3
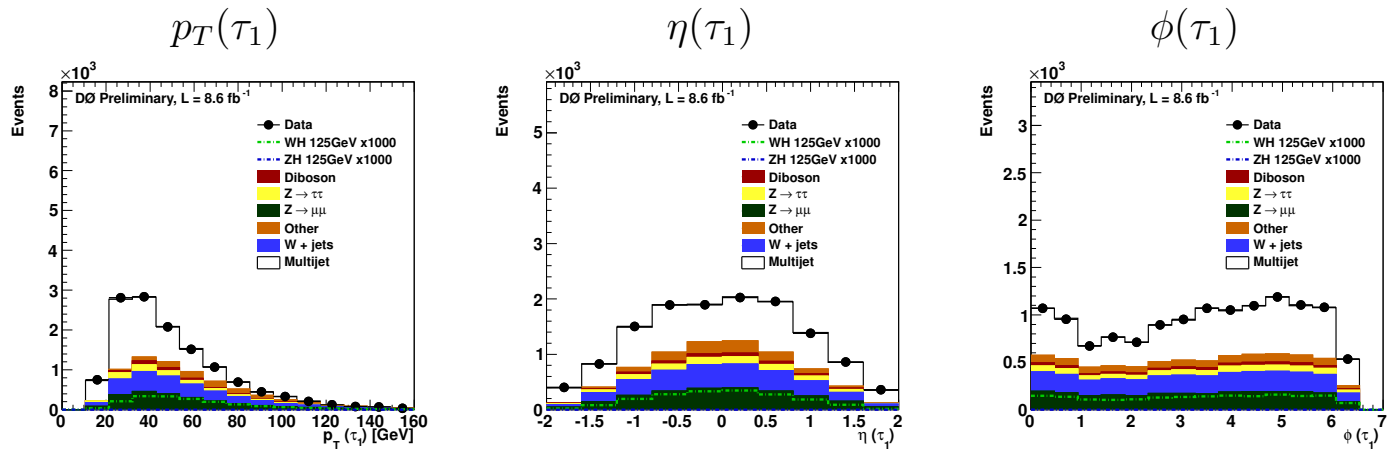

Figure 9.7: Data distributions at pre-selection for Type-1 tau leptons (top), Type2 tau leptons (middle) and Type-3 tau leptons (bottom), compared to the sum of the expected backgrounds from MC simulations and multijet background methods. The MC distribution of the associated production of a SM Higgs boson produced with a $Z$ and $W$ boson and a mass of $125 \mathrm{GeV}$, multiplied by a factor of 1000 , are superimposed. The 'Other' background sample contains the contribution from $Z \rightarrow e e$ and $t \bar{t}$ events. 


\begin{tabular}{l|c|ccc} 
& $\begin{array}{c}\text { Pre-selection } \\
\text { All Types }\end{array}$ & Type-1 & Type-2 & Type-3 \\
\hline \hline Data & 22859 & 1102 & 8417 & 13146 \\
$Z / \gamma^{*} \rightarrow \tau \tau$ & $1562 \pm 199$ & $60 \pm 8$ & $656 \pm 84$ & $784 \pm 100$ \\
$Z / \gamma^{*} \rightarrow \mu \mu$ & $3795 \pm 483$ & $152 \pm 19$ & $1250 \pm 159$ & $2353 \pm 299$ \\
$Z / \gamma^{*} \rightarrow \mu \mu$ & $12 \pm 2$ & $<1$ & $8 \pm 1$ & $2 \pm 0.3$ \\
$t \bar{t}$ & $1472 \pm 215$ & $19 \pm 3$ & $428 \pm 63$ & $1020 \pm 149$ \\
Diboson & $609 \pm 78$ & $17 \pm 3$ & $191 \pm 24$ & $396 \pm 50$ \\
$W+$ jet & $5628 \pm 707$ & $309 \pm 39$ & $3158 \pm 397$ & $2579 \pm 324$ \\
Multijet & $10588 \pm 272$ & $553 \pm 128$ & $4021 \pm 1006$ & $6011 \pm 1503$ \\
Total Bkg & $23665 \pm 1740$ & $1109 \pm 395$ & $8405 \pm 1732$ & $13141 \pm 2425$ \\
\hline Signal & & & & \\
125 GeV & & & & \\
$Z H$ & $2.39 \pm 0.30$ & $0.11 \pm 0.01$ & $0.98 \pm 0.12$ & $1.10 \pm 0.14$ \\
$W H$ & $4.35 \pm 0.55$ & $0.24 \pm 0.03$ & $2.11 \pm 0.27$ & $1.81 \pm 0.23$
\end{tabular}

Table 9.3: Predictions from the MC simulations and the data determined multijet backgrounds contribution compared to the data at pre-selection. The event yields are listed for all tau lepton types combined and split into Type-1, Type-2 and Type-3 tau leptons. The events are classified by the type of the highest $p_{T}$ tau lepton. The signal is given for the associated production of a SM Higgs boson of mass $125 \mathrm{GeV}, W H$ and $Z H$. Systematic uncertainties on the MC and multijet backgrond are given. The contribution from statistical uncertainties is taken to be negligible and not included.

while enhancing the signal region. The kinematic distributions after these additional selection criteria are implemented are shown in Figure 9.8 and Figure 9.9. These requirements and their motivation are described below.

1. $N N_{\tau}>0.75 / 0.75 / 0.95$ for tau lepton Type-1/Type-2/Type-2, respectively, for both tau leptons candidates.

The $N N_{\tau}$ output is required to be greater than 0.75 for Type- 1 and Type- 2 tau lepton candidates and greater than 0.95 for Type-3 tau lepton candidates. This requirement is applied to discriminate tau leptons from those events with misidentified tau leptons. It removes the majority of $W+$ jets and multijet events selected, while retaining the majority of the signal events. 
2. $\left|\eta_{\tau}\right|<1.5$ for both tau leptons candidates.

As can be seen in Figure 9.6, the signal events peak at low values of $\left|\eta_{\tau}\right|$, therefore this requirement can further isolate the region of phase space where the signal events are located.

3. $\mathbb{E}_{\mathrm{T}}>20 \mathrm{GeV}$.

It can be seen in Figure 9.6, that the multijet events peak at low values of $E_{\mathrm{T}}$ compared to the signal, therefore this requirement removes multijet events and improves the ratio of the number of selected signal events to the number of selected background events.

4. $|Q|=\left|\Sigma_{i} q_{i}\right|=\left|q_{\mu}+q_{\tau_{1}}+q_{\tau_{2}}\right|=1$.

As described in Section 7.8.2, for signal-like events it is expected that the sum of the electric charge, $Q$, of the three selected final state particles will be equal to one, for example $H W^{+} \rightarrow \tau^{+} \tau^{-} \mu^{+} \nu_{\mu}$. Whereas for the multijet all the selected particles are as likely to have the same electric charge $|Q|$ $=3$, as for $Q=1$. This can be seen in the distribution of $Q$ in Figure 7.11. Therefore placing a requirement on the electric charge of the final state particles increases the number of selected signal events in relation to the number of selected background events.

5. $M_{T}>20 \mathrm{GeV}$

This requirement on the transverse mass reduces the $Z / \gamma^{*} \rightarrow \tau \tau$ contribution which peaks at low values of $M_{T}$, and hence improves the signal to background ratio.

\subsubsection{Distributions at final selection}

The distribution of the data compared to predicted events from MC simulations and data determined multijet backgrounds, for the variables listed in Section 8.4.2 
are showed with all final selection requirements applied, in Figures 9.8 and 9.9. The signal samples, for a SM Higgs boson with mass $M_{H}=125 \mathrm{GeV}$, produced in association with a $W$ and $Z$ boson are superimposed, multiplied by a factor of 10. In Figure 9.9, the invariant mass of the two tau leptons $M\left(\tau_{1} \tau_{2}\right)$ displays a second peak composed mainly of diboson events at $M\left(\tau_{1} \tau_{2}\right)=90 \mathrm{GeV}$. This peak is caused by electrons that are misidentified as tau leptons.

The number of data, of predicted background events from MC simulation, the multijet contribution, and the dominant signals samples are listed in Table 9.4, for each final selection requirement. The $N N_{\tau}$ requirement removes the majority of the background events. The variable $S / \sqrt{B}$ which gives a representation of the sensitivity of this analysis, where $S$ is the total number of predicted signal events and $B$ is the total number of predicted background events. Table 9.5 shows the yields when split into tau lepton types. The events are classified by the tau lepton type of the highest $p_{T}$ tau lepton.

\subsection{Signal sensitivity}

The number of predicted SM Higgs boson signal events that have been selected by the final selection criteria, for each of the SM Higgs boson production mechanisms, are shown in Figure 9.10, as a function of $M_{H}$. The production mechanisms are, associated production $W H$ and $Z H$, gluon-gluon fusion production of a Higgs decaying as $H \rightarrow W W$ or $H \rightarrow Z Z$, and vector boson fusion. The dominant production mechanism is the associated $W H$ production. A comparison of the distribution of the $W H$ signal for $M_{H}=120,140,160$, and $180 \mathrm{GeV}$ is shown in Figure 9.11, comparing the $\mathbb{E}_{\mathrm{T}}, M\left(\tau_{1}, \tau_{2}\right)$, and $\Delta R\left(\tau_{1}, \tau_{2}\right)$ distributions. For $M_{H}=130 \mathrm{GeV}$ the $H \rightarrow \tau \tau$ decay dominates. Between 130 and $160 \mathrm{GeV}$ this analysis is sensitive to both $H \rightarrow \tau \tau$ and $H \rightarrow W W$ decay and above $M_{H}=$ $160 \mathrm{GeV} H \rightarrow W W$ dominates, with a small contribution from $H \rightarrow Z Z$. This 


\begin{tabular}{|c|c|c|c|c|c|c|}
\hline & \multirow{2}{*}{$\begin{array}{c}\text { Pre- } \\
\text { selection }\end{array}$} & \multicolumn{5}{|c|}{ Final selection requirements applied } \\
\hline & & $N N_{\tau}$ & $N N_{\tau}, E_{\mathrm{T}}$ & $\begin{array}{l}N N_{\tau} \\
E_{\mathrm{T}}, \eta_{\tau}\end{array}$ & $\begin{array}{c}N N_{\tau}, E_{\mathrm{T}} \\
\eta_{\tau},|Q|\end{array}$ & $\begin{array}{c}N N_{\tau}, E_{\mathrm{T}}, \\
\eta_{\tau},|Q|, M_{T}\end{array}$ \\
\hline Data & 22859 & 104 & 77 & 50 & 46 & 22 \\
\hline$Z / \gamma^{*} \rightarrow \tau \tau$ & $1563 \pm 199$ & $34.2 \pm 4.4$ & $19.8 \pm 2.5$ & $15.5 \pm 1.8$ & $15.5 \pm 2.0$ & $5.6 \pm 0.7$ \\
\hline$Z / \gamma^{*} \rightarrow \mu \mu$ & $3795 \pm 483$ & $19.0 \pm 2.4$ & $7.9 \pm 1.0$ & $6.6 \pm 0.8$ & $6.3 \pm 0.8$ & $4.4 \pm 0.6$ \\
\hline$Z / \gamma^{*} \rightarrow \mu \mu$ & $12 \pm 2$ & $1.7 \pm 0.2$ & $0.9 \pm 0.1$ & $0.5 \pm 0.1$ & $0.5 \pm 0.1$ & $0.3 \pm 0.04$ \\
\hline & $1472 \pm 215$ & $2.0 \pm 0.3$ & $1.9 \pm 0.3$ & $1.6 \pm 0.2$ & $1.6 \pm 0.2$ & $1.4 \pm 0.2$ \\
\hline Diboson & $609 \pm 78$ & $17.7 \pm 2.6$ & $14.7 \pm 2.1$ & $10.1 \pm 1.5$ & $10.0 \pm 1.5$ & $9.0 \pm 1.3$ \\
\hline$W+$ jet & $5628 \pm 707$ & $8.7 \pm 1.1$ & $7.6 \pm 1.0$ & $5.8 \pm 0.7$ & $5.5 \pm 0.7$ & $5.4 \pm 0.7$ \\
\hline Multijet & $10588 \pm 272$ & $5.0 \pm 1.1$ & $2.4 \pm 0.5$ & $<0.46$ & $<0.52$ & $<0.47$ \\
\hline Total Bkg & $23666 \pm 1740$ & $88.3 \pm 12.1$ & $55.1 \pm 7.5$ & $40.4 \pm 5.6$ & $39.2 \pm 5.8$ & $26.1 \pm 4.0$ \\
\hline $\begin{array}{l}\text { Signal } \\
125 \mathrm{GeV}\end{array}$ & & & & & & \\
\hline$Z H$ & $2.39 \pm 0.30$ & $0.32 \pm 0.04$ & $0.26 \pm 0$ & $0.21 \pm 0.03$ & $0.21 \pm 0.03$ & $0.17 \pm 0.02$ \\
\hline$W H$ & $4.34 \pm 0.55$ & $0.79 \pm 0.10$ & $0.69 \pm 0.08$ & $0.60 \pm 0.7$ & $0.59 \pm 0.07$ & $0.52 \pm 0.06$ \\
\hline$S / \sqrt{B}$ & 0.04 & 0.12 & 0.13 & 0.13 & 0.13 & 0.13 \\
\hline
\end{tabular}

Table 9.4: Predicted events from MC simulation and multijet background methods compared to the observation in data at pre-selection and after each final selection requirement. The signal is shown for the associated production of a SM Higgs boson with a mass of $M_{H}=125 \mathrm{GeV}$ for the channels $W H$ and $Z H$. The ratio $S / \sqrt{B}$ is also given. Systematic uncertainties on the MC and multijet are included. The contribution from statistical uncertainties is taken to be negligible and not included.

\begin{tabular}{l|c|ccc} 
& All Types & Type-1 & Type-2 & Type-3 \\
\hline \hline Data & 22 & 2 & 15 & 5 \\
$Z / \gamma^{*} \rightarrow \tau \tau$ & $5.56 \pm 0.71$ & $0.75 \pm 0.10$ & $3.91 \pm 0.50$ & $0.91 \pm 0.12$ \\
$Z / \gamma^{*} \rightarrow \mu \mu$ & $4.42 \pm 0.56$ & $0.70 \pm 0.09$ & $3.24 \pm 0.41$ & $0.49 \pm 0.06$ \\
$Z / \gamma^{*} \rightarrow \mu \mu$ & $0.31 \pm 0.04$ & $<0.01$ & $0.26 \pm 0.03$ & $0.05 \pm 0.01$ \\
$t \bar{t}$ & $1.43 \pm 0.21$ & $0.27 \pm 0.04$ & $1.07 \pm 0.16$ & $0.07 \pm 0.03$ \\
Diboson & $9.01 \pm 1.31$ & $0.41 \pm 0.06$ & $7.01 \pm 0.10$ & $1.58 \pm 0.23$ \\
$W+$ jet & $5.38 \pm 0.66$ & $0.62 \pm 0.08$ & $3.20 \pm 0.40$ & $1.54 \pm 0.19$ \\
Multijet & $<0.47$ & $<0.04$ & $<0.32$ & $<0.11$ \\
Total Bkg & $26.11 \pm 3.96$ & $2.75 \pm 0.37$ & $18.67 \pm 1.2$ & $4.63 \pm 0.48$ \\
\hline Signal 125 GeV & & & & \\
$Z H$ & $0.17 \pm 0.02$ & $0.02 \pm 0.002$ & $0.13 \pm 0.02$ & $0.02 \pm 0.002$ \\
$W H$ & $0.52 \pm 0.06$ & $0.05 \pm 0.01$ & $0.40 \pm 0.05$ & $0.06 \pm 0.01$
\end{tabular}

Table 9.5: Predicted MC events and multijet backgrounds contributions compared to the data at final selection, for all tau lepton types combined and separated into types. The signal is shown for the associated production of a SM Higgs boson with $M_{H}=125 \mathrm{GeV}$ in the $W H$ and $Z H$ channels. 

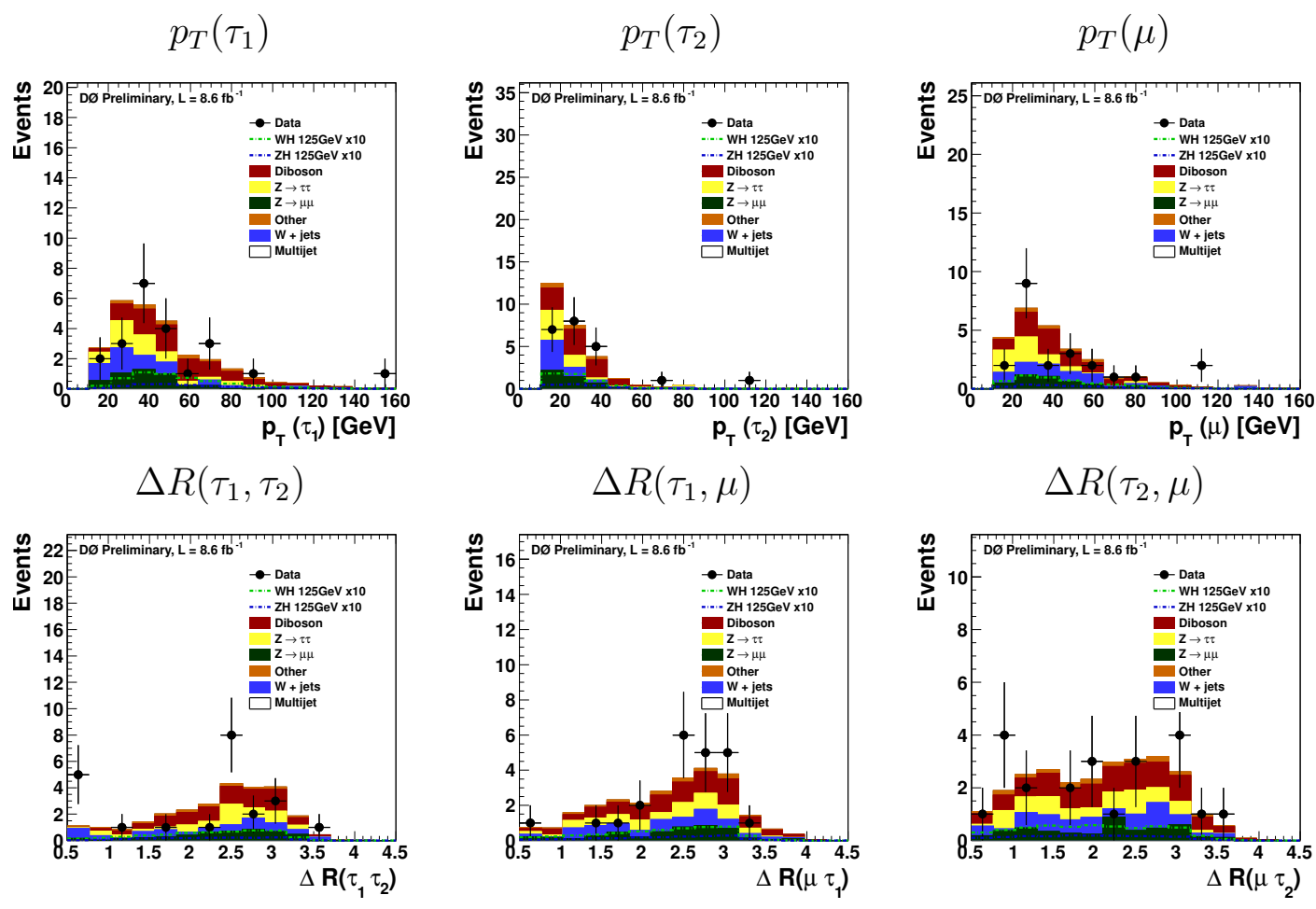

$N_{\mu}$

$N_{\tau}$
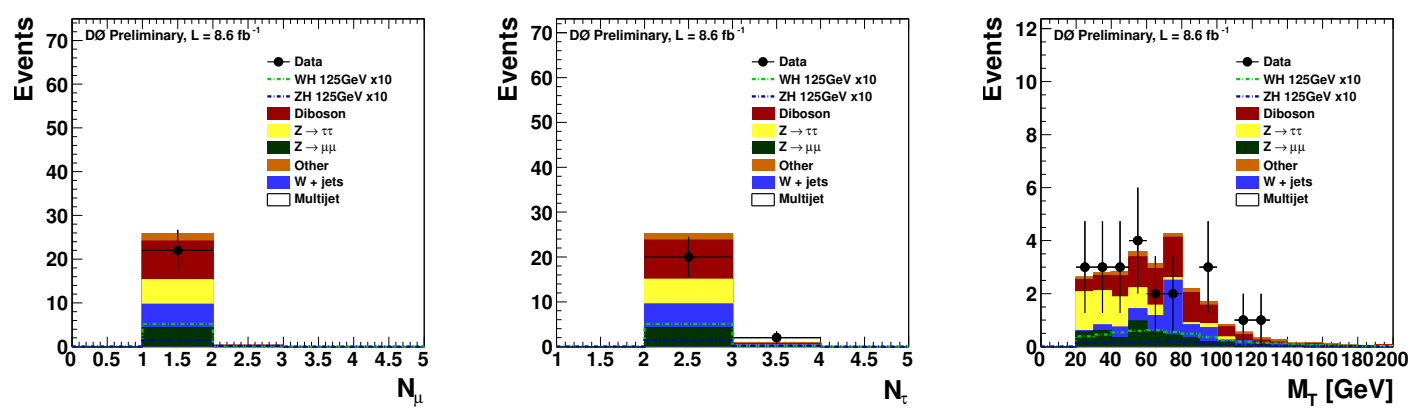

Figure 9.8: Data distributions at final selection compared to the sum of the expected backgrounds from MC simulations and multijet background methods. The MC simulation of the associated production of a SM Higgs boson with $M_{H}=125 \mathrm{GeV}$ in the $W H$ and $Z H$ channels, multiplied by a factor of 10 , are superimposed. The 'Other' background sample contains the contributions from $Z \rightarrow e e$ and $t \bar{t}$ events. 
$E_{\mathrm{T}}$

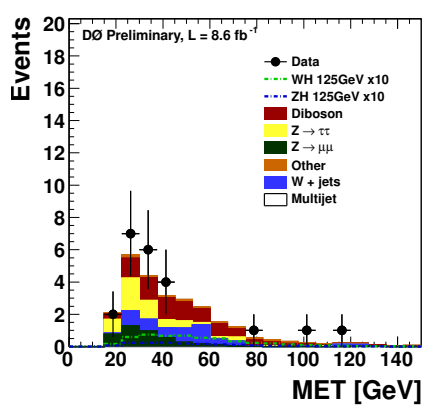

$\eta\left(\tau_{1}\right)$

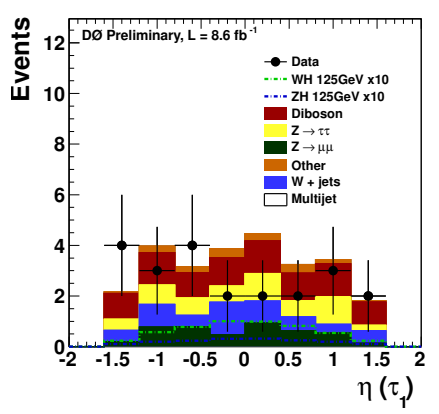

$\phi\left(\tau_{1}\right)$

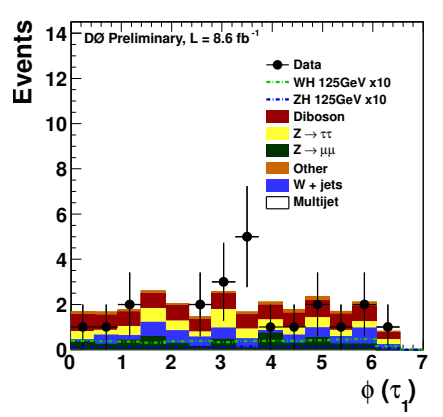

$M\left(\mu, \tau_{1}, \tau_{2}\right)$

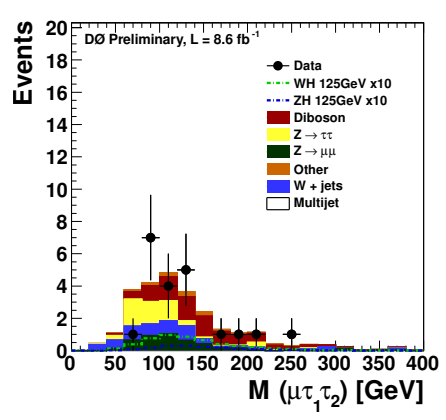

$\eta\left(\tau_{2}\right)$

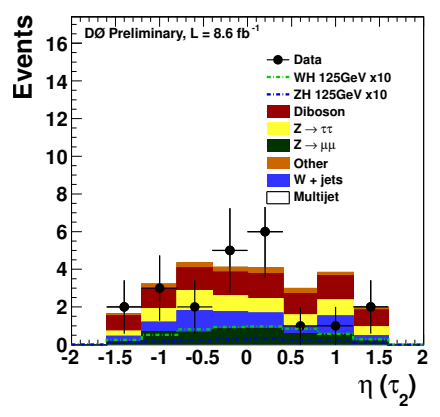

$\phi\left(\tau_{2}\right)$

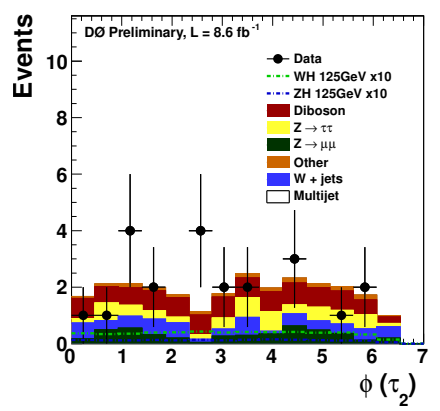

$M\left(\tau_{1}, \tau_{2}\right)$

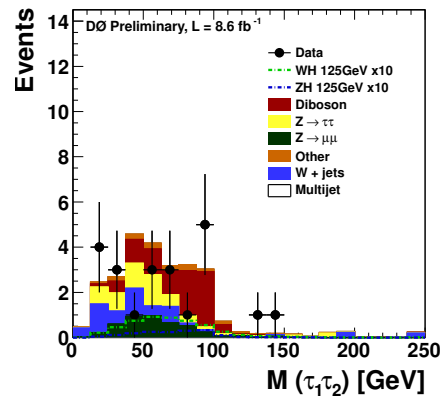

$\eta(\mu)$

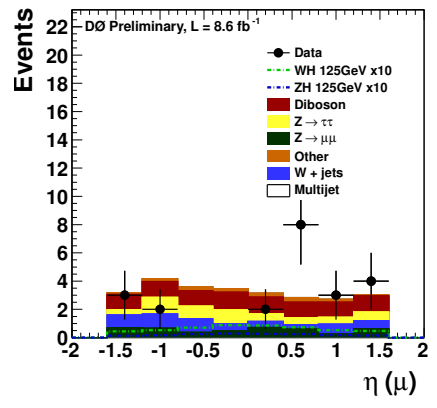

$\phi(\mu)$

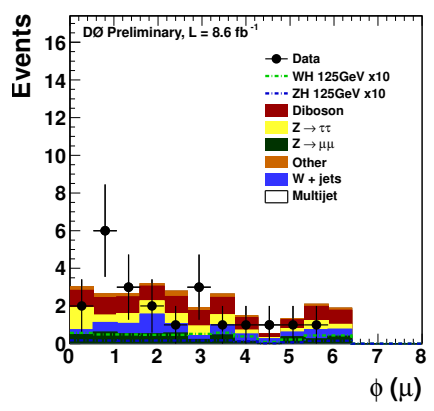

Figure 9.9: Data distributions at final selection compared to the sum of the expected backgrounds from MC simulations and multijet background methods. The MC simulation of the associated production of a SM Higgs boson with $M_{H}=125 \mathrm{GeV}$ in the $W H$ and $Z H$ channels, multiplied by a factor of 10 , are superimposed. The 'Other' background sample contains the contributions from $Z \rightarrow e e$ and $t \bar{t}$ events. 


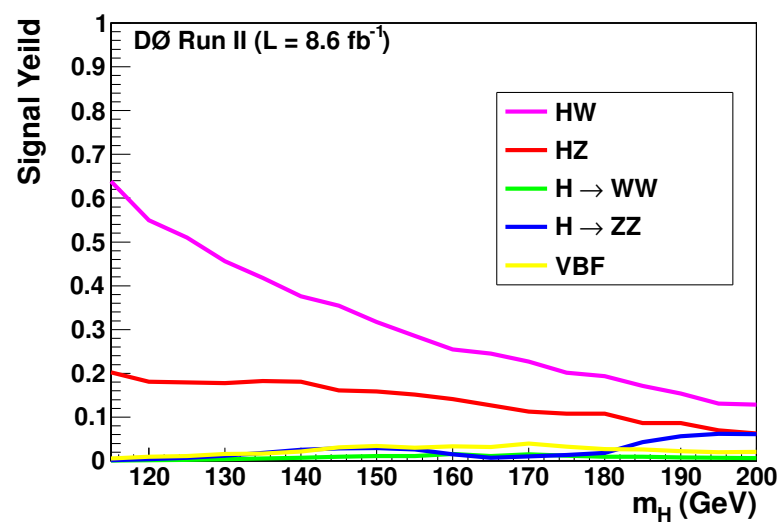

Figure 9.10: The number of signal events at final selection from the Higgs boson production modes at the Tevatron, as a function of the SM Higgs boson mass. The production mechanisms shown are, associated production $W H$ and $Z H$, the gluon-gluon fusion production of a Higgs decaying as $H \rightarrow W W$ or $H \rightarrow Z Z$, and vector boson fusion, VBF.
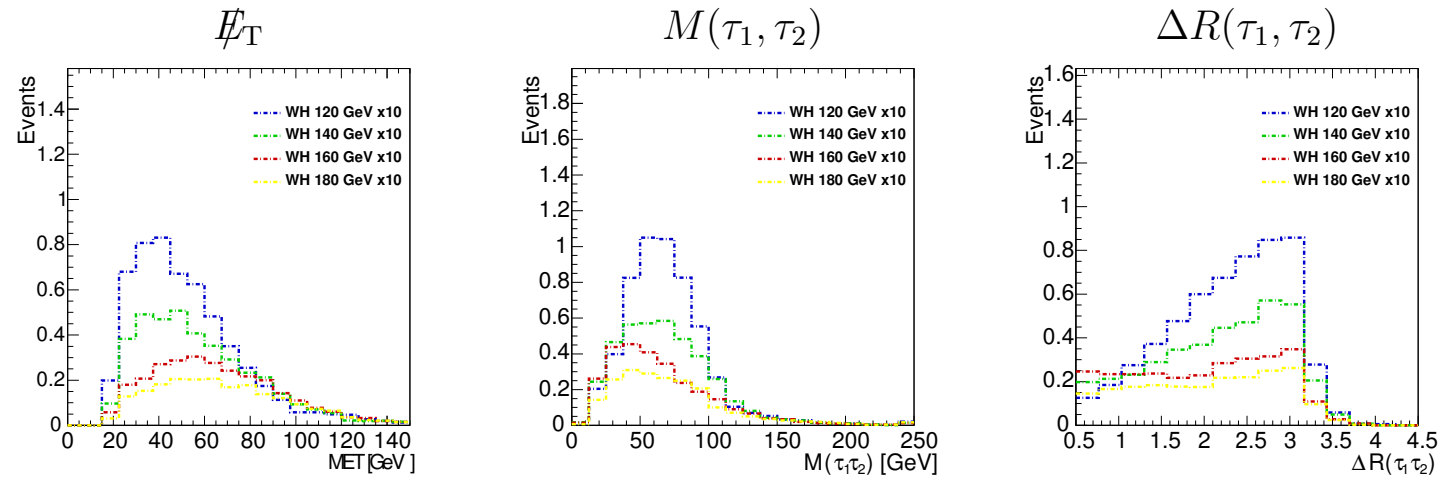

Figure 9.11: The distributions of the selected signal events at final selection, for $W H$ associated production of a Higgs boson signal, for $M_{H}=120,140,160$, and $180 \mathrm{GeV}$, scaled by a factor of 10 . 
is as expected from the SM Higgs branching ratios as shown in Figure 2.3. This analysis is less sensitive to Higgs bosons that decay to a $W$ or $Z$ boson pair as they have several additional decay channels that this analysis is not sensitive too.

\subsection{Multivariate analysis}

To achieve a significant separation between signal and background events, Boosted Decision Trees, BDTs, are used, within the TMva framework [65]. The BDTs are trained with a selection of the most discriminating variables to create a final discriminant distribution. Multivariate analysis methods are described in detail in Section 7.9. The diboson background events have the most similar shape to the SM Higgs signal sample. Therefore, this is the background that is most difficult to discriminate from signal events. For this reason, two separate BDTs are used, BDT Pass 1 is trained against all backgrounds samples except the diboson sample and reduces the contributions from the non-diboson background events considerably. All events that pass the BDT Pass 1 with a value greater than the value that was determined to be the optimal background to signal rejection value, are then used to train a second BDT, BDT Pass 2. Throughout this section all distributions and yields will be for a SM Higgs boson of mass $M_{H}=125 \mathrm{GeV}$.

\subsubsection{BDT Pass 1}

The first BDT, BDT Pass 1, is trained against all predicted MC background events except for the diboson MC samples. A BDT is trained for each of the Higgs boson mass points from $M_{H}=100$ to $200 \mathrm{GeV}$, in intervals of $5 \mathrm{GeV}$, using the input variables listed in this section. The following TMVA training configurations, which differ from the defaults, listed in Table 7.9, are used; Number 
of tree: 140, MaxDepth: 6, BoostType: Bagging, UseYesNoLeaf: False, UseRandomizedTrees: True.

\section{Input variables}

Nine variables are used for training BDT Pass 1 . The distributions that are used in the training, but are not shown in Figure 9.8 or Figure 9.9 are shown in Figure 9.12. The nine variables are:

1. $p_{T}\left(\tau_{1}\right)$ : transverse momentum of the highest $p_{T}$ tau lepton.

2. $p_{T}\left(\tau_{2}\right)$ : transverse momentum of the second highest $p_{T}$ tau lepton.

3. $p_{T}(\mu)$ : transverse momentum of the highest $p_{T}$ muon.

4. $M\left(\mu, \tau_{1}, \tau_{2}\right)$ : invariant mass of the three reconstructed leptons.

5. $M\left(\tau_{1}, \tau_{2}\right)$ : invariant mass of the two reconstructed tau leptons.
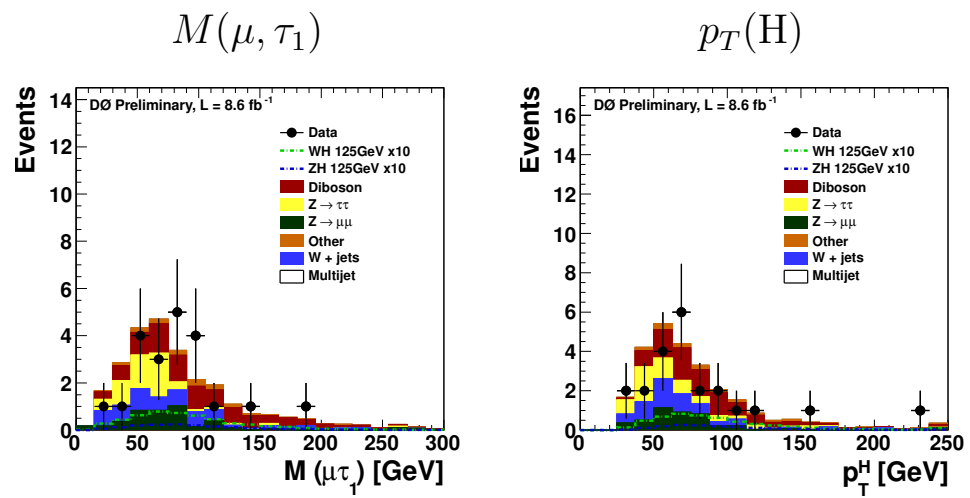

Figure 9.12: Data distributions at final selection compared to the sum of the expected backgrounds from MC simulations and multijet estimation methods, for the $M\left(\mu, \tau_{1}\right)$ and the $p_{T}(\mathrm{H})$ distributions used as inputs to BDT Pass 1 . The MC simulation of the associated production of a SM Higgs boson with $M_{H}=125$ $\mathrm{GeV}$ in the $Z H$ and $W H$ channel, multiplied by a factor of 10 , are superimposed. The 'Other' background sample contains the contributions from $Z \rightarrow e e$ and $t \bar{t}$. 
6. $M\left(\tau_{1}, \mu\right)$ : invariant mass of the highest $p_{T}$ tau lepton and the leading $p_{T}$ muon.

7. $\mathbb{E}_{\mathrm{T}}$ : missing transverse energy.

8. $p_{t}(\mathrm{H}): p_{T}$ of the pair of leptons with opposite electric charge from the three reconstructed leptons, which are assumed to originate from the Higgs boson decay.

9. $M_{T}$ : The transverse mass.

The distribution of signal and background training samples as used by TMVA for the the nine input variables is shown in Figure 9.13. The signal training sample consists of the summed contribution from all the SM Higgs boson samples as described in Section 9.2.2 ( $W H, H Z, \mathrm{VBF}, H W W$ and $H Z Z)$. The background training sample consists of the sum of all the SM background samples as described in Section 7.3, at final selection. All reweightings and normalizations are included. The background training sample can be seen to suffer from low statistics.

\section{TMVA input studies}

The correlation matrices for the nine input variables for both the signal and background training samples are shown in Figure 9.14. The majority of variables show some positive correlation, with more variables showing stronger correlations for the background training sample than for the signal training sample. Figure 9.15 shows the overtraining distribution, as described in Section 7.9.3. The background samples can be seen to suffer from overtraining, whereas this does not affect the signal. This overtraining will limit the discriminating power of the output of the BDT Pass 1. The overtraining arises from the fluctuations due to low background MC sample statistics, as can be seen in Figure 9.13. These fluctuations can be as dominant as real features in the distributions, therefore 

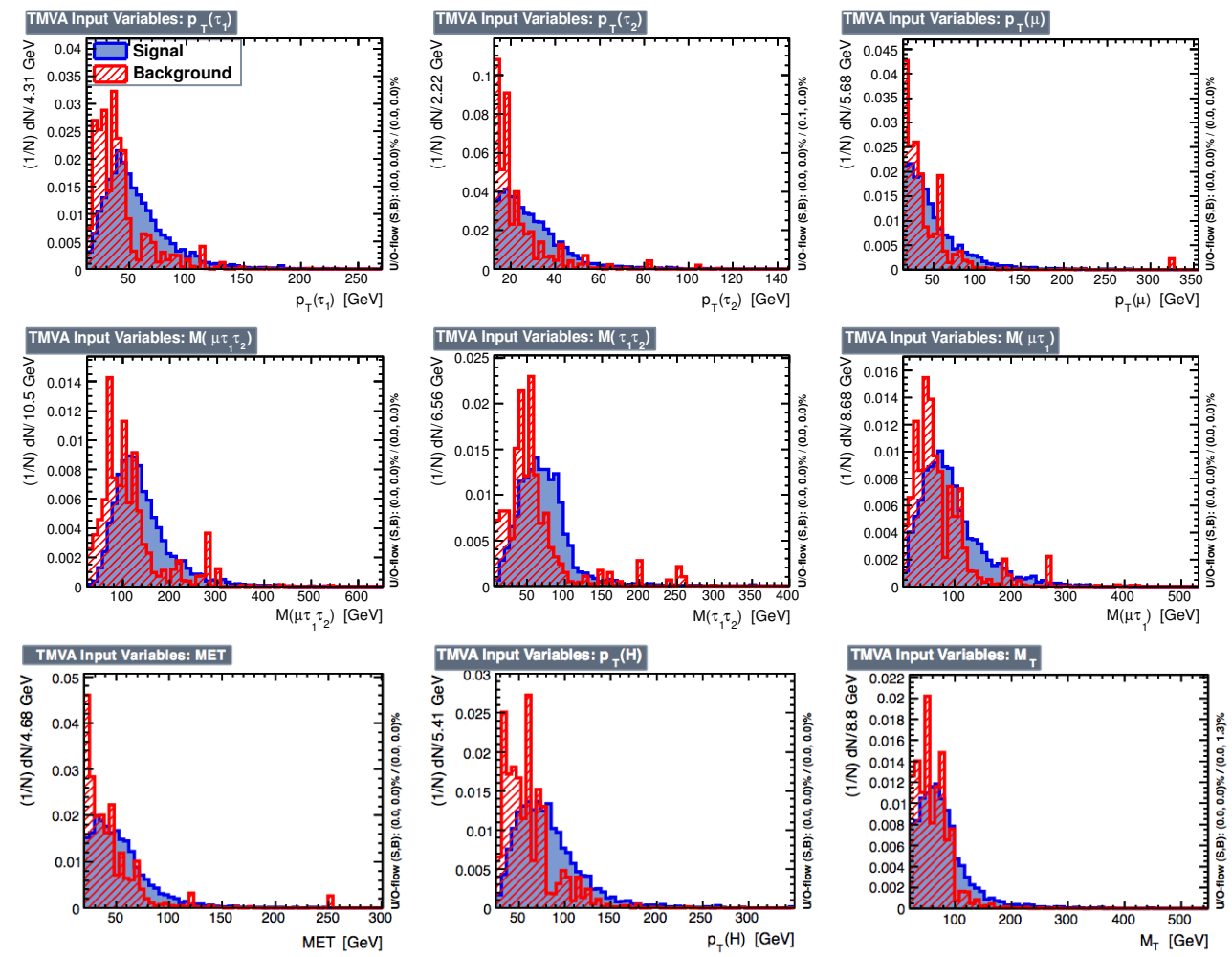

Figure 9.13: The signal and background separation distributions for the nine variables used to train the BDT Pass 1 for a Higgs boson mass of $M_{H}=125 \mathrm{GeV}$ as processed by TMVA. From top right to bottom left the following variables are shown, $p_{T}\left(\tau_{1}\right), p_{T}\left(\tau_{2}\right), p_{T}(\mu), M\left(\mu \tau_{1} \tau_{2}\right), M\left(\tau_{1} \tau_{2}\right), M\left(\tau_{1} \mu\right), E_{\mathrm{T}}, p_{t}(H)$ and $M_{T}$. The signal test sample has been renormalized for comparison with the background sample.

TMVA will train on them as well on real shape differences in the distribution of the signal and background test samples.

\section{BDT Pass 1 output distribution}

The BDT Pass 1 output discriminant distributions is shown in Figure 9.16. The diboson background MC sample and the signal samples are seen to peak at one, with the other background peaking towards lower values. The BDT discriminates well against $Z / \gamma^{*} \rightarrow \tau \tau$ and $W+$ jets MC samples. The BDT Pass 1 outputs for all SM Higgs boson mass points studied are available in Appendix A. 


\section{Correlation Matrix (signal)}

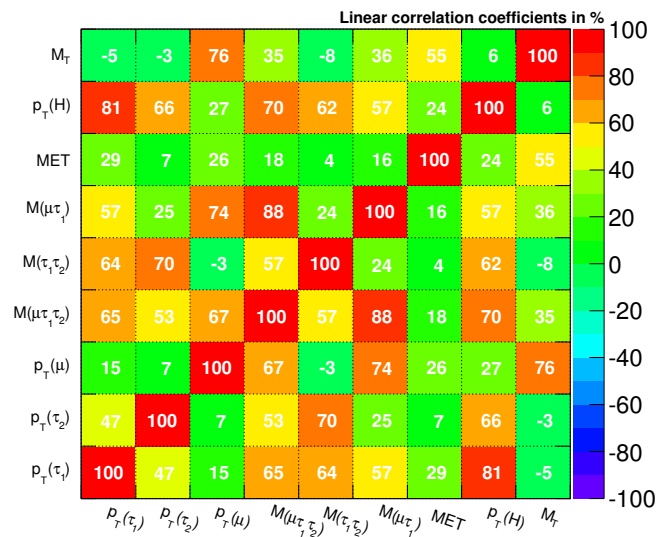

\section{Correlation Matrix (background)}

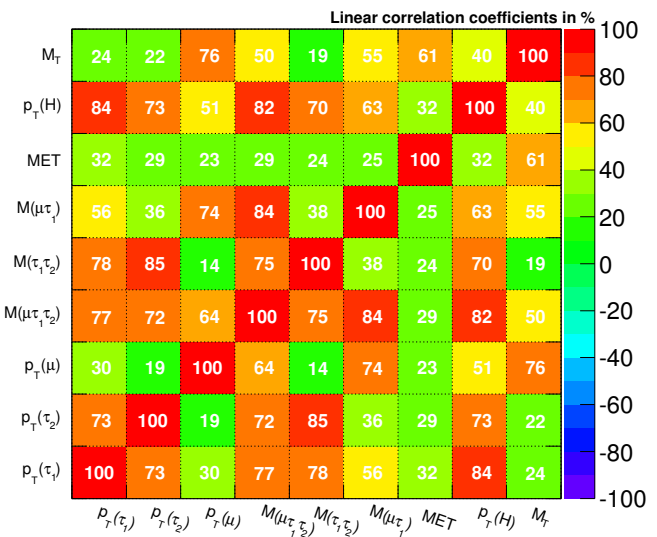

Figure 9.14: The correlation matrices for BDT Pass 1, for the signal (left) and background (right) training samples for SM Higgs boson mass $M_{H}=125 \mathrm{GeV}$, showing the linear correlations between the input variables used by TMVA to train the BDT Pass 1.

\subsubsection{BDT Pass 1 selection requirement}

The majority of the non-diboson backgrounds can be removed by placing a selection requirement on the output of BDT Pass 1, creating a MC sample dominated by diboson background events. This enables a second BDT to be specifically trained on the diboson background events, for which the discriminating distribution shapes closely resembles those of the SM Higgs boson sample.

TMVA calculates the efficiency of the signal and background rejection and determines the optimal value at which the majority of the background can be rejected while retaining the majority of the signal. All events which have a BDT Pass 1 output greater than this optimal value are retrained with a second BDT, BDT Pass 2. The optimal rejection values for BDT Pass 1 are listed in Table 9.6. The data and predicted MC event yields, after this criteria has been applied are given in Table 9.7. 


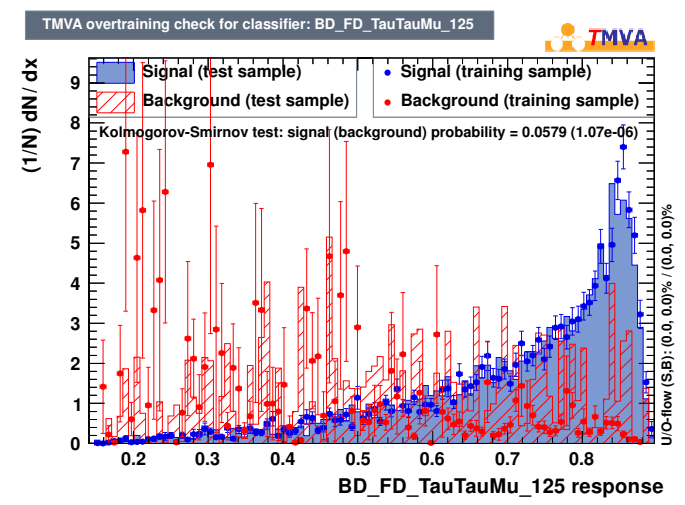

Figure 9.15: The signal versus background overtraining distribution for the BDT Pass 1 trained against all MC samples except the diboson samples, for a Higgs boson mass of $M_{H}=125 \mathrm{GeV}$. Showing the signal (blue) and background (red) samples for both the training sample (dots) and and testing sample (filled histogram).

\begin{tabular}{l|ccccccc}
$M_{H}[\mathrm{GeV}]$ & 100 & 105 & 110 & 115 & 120 & 125 & 130 \\
BDT Pass 1 criteria & 0.698 & 0.680 & 0.738 & 0.657 & 0.705 & 0.744 & 0.758 \\
\hline$M_{H}[\mathrm{GeV}]$ & 135 & 140 & 145 & 150 & 155 & 160 & 165 \\
BDT Pass 1 criteria & 0.736 & 0.750 & 0.704 & 0.763 & 0.783 & 0.694 & 0.746 \\
\hline$M_{H}[\mathrm{GeV}]$ & 170 & 175 & 180 & 185 & 190 & 195 & 200 \\
BDT Pass 1 criteria & 0.707 & 0.699 & 0.763 & 0.757 & 0.760 & 0.737 & 778
\end{tabular}

Table 9.6: The optimal signal and background rejection values as determined by TMVA for the BDT Pass 1, given per mass point.

\subsubsection{BDT Pass 2}

BDT Pass 2 is trained against all background samples and is trained for each $M_{H}=100$ to $200 \mathrm{GeV}$, in intervals of $5 \mathrm{GeV}$. The following Tmva training configurations which are different from the defaults, as listed in Table 7.9 , are used; MaxDepth: 13, BoostType: Bagged, UseYesNoLeaf: False, UseRandomizedTrees: True. 


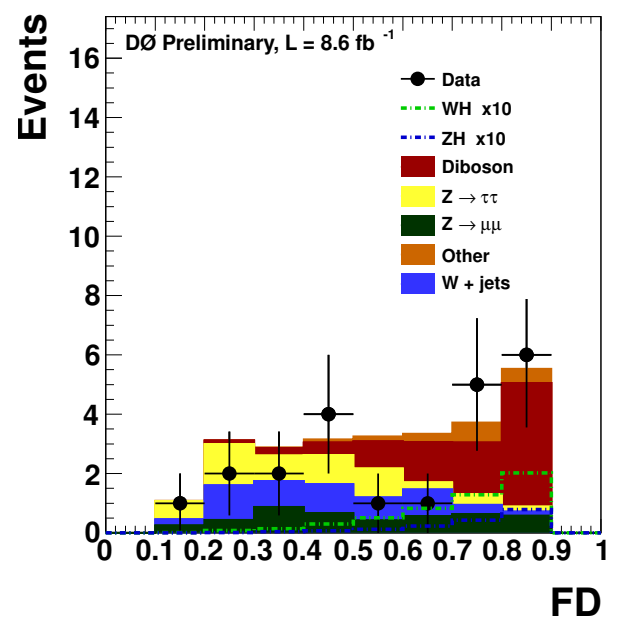

Figure 9.16: The observed data compared to predicted MC background events for the BDT Pass 1 distribution for a $125 \mathrm{GeV}$ SM Higgs boson. The MC simulation of the associated production for a SM Higgs boson with $M_{H}=125 \mathrm{GeV}$, in the $Z H$ and $W H$ channels, multiplied by a factor of 10 , are superimposed. The 'Other' background sample contains the contribution from $Z \rightarrow e e$, and $t \bar{t}$ events.

\section{Input variables}

The distribution of the data compared to the predicted MC background events for the four variables used for the training of the BDT Pass 2 are shown in Figure 9.17. Good agreement between the data and the predicted MC events after the BDT Pass 1 selection requirement is seen for the events remaining, which are dominated by diboson events. The distributions of the observed data and predicted MC events for other $M_{H}$ are seen to show a similar agreement [109]. The four input variables used in the BDT Pass 2 training are:

1. $p_{T}\left(\tau_{1}\right)$ : transverse momentum of the highest $p_{T}$ tau lepton.

2. $M\left(\tau_{1}, \tau_{2}\right)$ : invariant mass of the two reconstructed tau leptons.

3. $\mathbb{E}_{\mathrm{T}}$ : missing transverse energy.

4. $M_{T}$ : transverse mass. 


\begin{tabular}{l|cccccc} 
Mass $[\mathrm{GeV}]$ & 100 & 110 & 120 & 130 & 140 & 150 \\
\hline \hline Data & 6 & 3 & 8 & 5 & 6 & 6 \\
$Z / \gamma^{*} \rightarrow \tau \tau$ & $0.39 \pm 0.05$ & $0.11 \pm 0.01$ & $0.47 \pm 0.06$ & $0.25 \pm 0.03$ & $0.41 \pm 0.05$ & $0.22 \pm 0.03$ \\
$Z / \gamma^{*} \rightarrow \mu \mu$ & $0.70 \pm 0.09$ & $0.43 \pm 0.05$ & $0.71 \pm 0.09$ & $0.94 \pm 0.12$ & $0.43 \pm 0.05$ & $0.68 \pm 0.09$ \\
$Z / \gamma^{*} \rightarrow \mu \mu$ & $0.13 \pm 0.02$ & $0.13 \pm 0.02$ & $0.10 \pm 0.01$ & $0.11 \pm 0.01$ & $0.08 \pm 0.01$ & $0.12 \pm 0.02$ \\
$t \bar{t}$ & $0.71 \pm 0.10$ & $0.58 \pm 0.08$ & $0.71 \pm 0.10$ & $0.67 \pm 0.10$ & $0.76 \pm 0.11$ & $0.67 \pm 0.10$ \\
Diboson & $5.25 \pm 0.67$ & $4.58 \pm 0.58$ & $5.61 \pm 0.71$ & $5.15 \pm 0.66$ & $4.82 \pm 0.61$ & $4.61 \pm 0.59$ \\
$W+$ jet & $0.37 \pm 0.05$ & $0.57 \pm 0.07$ & $0.39 \pm 0.05$ & $0.29 \pm 0.04$ & $0.27 \pm 0.03$ & $0.42 \pm 0.05$ \\
Total Bkg & $7.54 \pm 0.98$ & $6.41 \pm 0.81$ & $7.99 \pm 1.01$ & $7.41 \pm 0.96$ & $6.79 \pm 0.86$ & $6.73 \pm 0.98$ \\
\hline Signal & \multicolumn{7}{c}{} & & & & \\
$Z H$ & $0.11 \pm 0.01$ & $0.09 \pm 0.01$ & $0.11 \pm 0.01$ & $0.10 \pm 0.01$ & $0.12 \pm 0.01$ & $0.09 \pm 0.01$ \\
$W H$ & $0.43 \pm 0.05$ & $0.32 \pm 0.04$ & $0.31 \pm 0.04$ & $0.23 \pm 0.03$ & $0.21 \pm 0.03$ & $0.17 \pm 0.02$
\end{tabular}

\begin{tabular}{l|ccccc} 
Mass [GeV] & 160 & 170 & 180 & 190 & 200 \\
\hline \hline Data & 7 & 5 & 6 & 7 & 4 \\
$Z / \gamma^{*} \rightarrow \tau \tau$ & $0.27 \pm 0.03$ & $0.19 \pm 0.02$ & $0.20 \pm 0.03$ & $0.22 \pm 0.03$ & $0.20 \pm 0.03$ \\
$Z / \gamma^{*} \rightarrow \mu \mu$ & $0.61 \pm 0.78$ & $0.41 \pm 0.05$ & $0.34 \pm 0.04$ & $0.37 \pm 0.05$ & $0.60 \pm 0.07$ \\
$Z / \gamma^{*} \rightarrow \mu \mu$ & $0.10 \pm 0.01$ & $0.09 \pm 0.01$ & $0.06 \pm 0.01$ & $0.16 \pm 0.02$ & $0.14 \pm 0.02$ \\
$t \bar{t}$ & $0.91 \pm 0.13$ & $0.88 \pm 0.13$ & $0.73 \pm 0.11$ & $0.79 \pm 0.12$ & $0.76 \pm 0.11$ \\
Diboson & $4.47 \pm 0.60$ & $4.67 \pm 0.60$ & $3.98 \pm 0.51$ & $4.73 \pm 0.60$ & $4.47 \pm 0.57$ \\
$W+$ jet & $0.18 \pm 0.02$ & $0.75 \pm 0.10$ & $0.51 \pm 0.07$ & $0.40 \pm 0.05$ & $0.38 \pm 0.05$ \\
Total Bkg & $6.53 \pm 1.57$ & $6.89 \pm 0.91$ & $5.82 \pm 0.77$ & $6.67 \pm 0.87$ & $6.54 \pm 0.85$ \\
\hline Signal & \multicolumn{1}{c}{} & & & \\
$Z H$ & $0.10 \pm 0.01$ & $0.08 \pm 0.01$ & $0.07 \pm 0.01$ & $0.06 \pm 0.01$ & $0.05 \pm 0.01$ \\
$W H$ & $0.18 \pm 0.02$ & $0.17 \pm 0.02$ & $0.12 \pm 0.01$ & $0.11 \pm 0.01$ & $0.09 \pm 0.01$
\end{tabular}

Table 9.7: Prediction from MC compared to the observation in data after the selection requirement on the BDT Pass 1 . The signal is shown for the associated production of a Higgs boson in the $W H$ and $Z H$ channels. Systematic uncertainties on the $\mathrm{MC}$ and multijet are given. The contribution from statistical uncertainties is taken to be negligible and not included.

The distribution of the four input variables is shown in Figure 9.18, for the training samples of signal and background events as used as inputs to TMVA for training of the BDT Pass 2. The signal training sample consists of a summed sample of all the SM Higgs boson samples as described in Section 9.2.2 ( $W H, H Z$, VBF, $H W W$, and $H Z Z$ ), which pass the BDT Pass 1 selection criteria, and the background training sample of a summed sample of all the background sample as described in Section 7.3, which pass the BDT Pass 1 selection criteria. All reweightings and normalizations are included. The background test sample can 

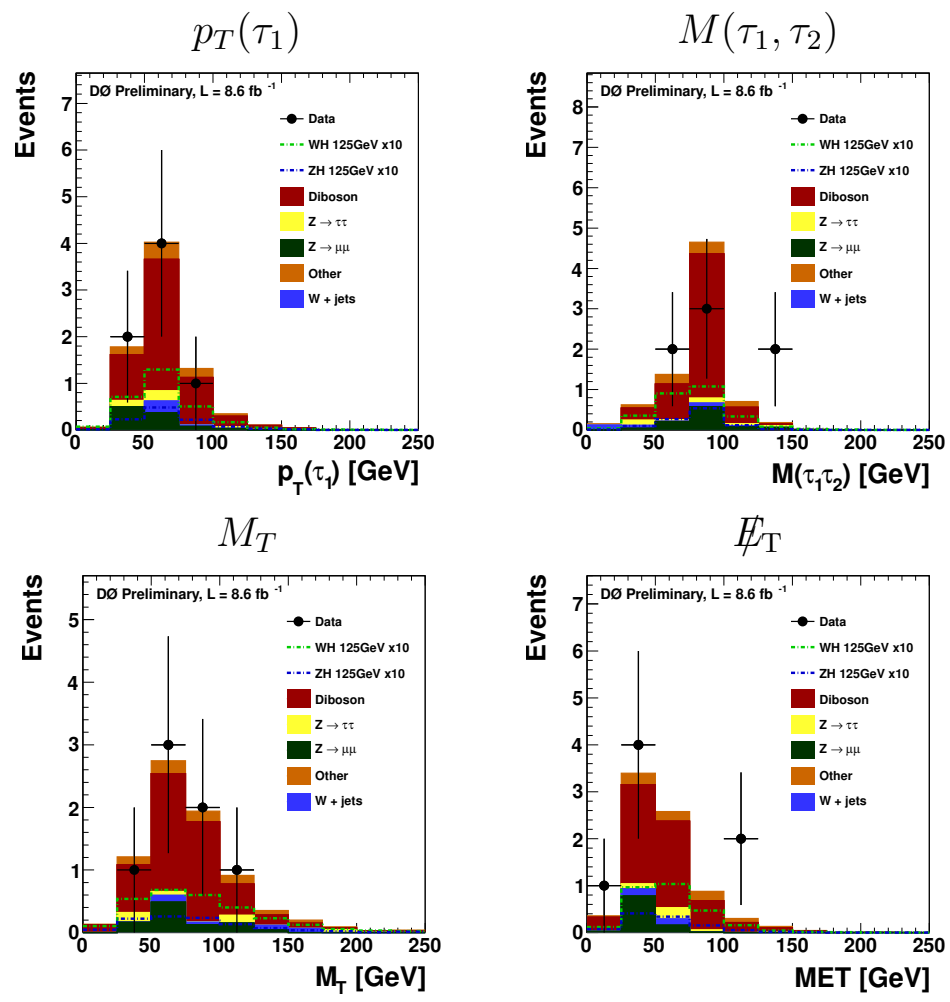

Figure 9.17: Comparison of the data with the predicted MC events, for the input distributions for the BDT Pass 2 for the $125 \mathrm{GeV}$ training point. The MC simulation of the associated production for a $125 \mathrm{GeV}$ SM Higgs boson with a $Z$ and $W$ boson, multiplied by a factor of 10, are superimposed. The 'Other' background sample contains the contributions from $Z \rightarrow e e$, and $t \bar{t}$ events.

be seen to suffer from low MC statistics.

\section{TMVA input studies}

The correlation matrices for a $M_{H}=125 \mathrm{GeV}$ for both the signal and background samples are shown in Figure 9.19, for BDT Pass 2. None of the input distributions used for training are strongly correlated and the correlations are consistent for signal and background events. The overtraining distribution comparing the TMVA training and testing samples, is shown in Figure 9.20. Overtraining is observed for the background sample, as for BDT Pass 1. This is again caused by the fluctuations due to low statistics as can be seen in Figure 9.18. 

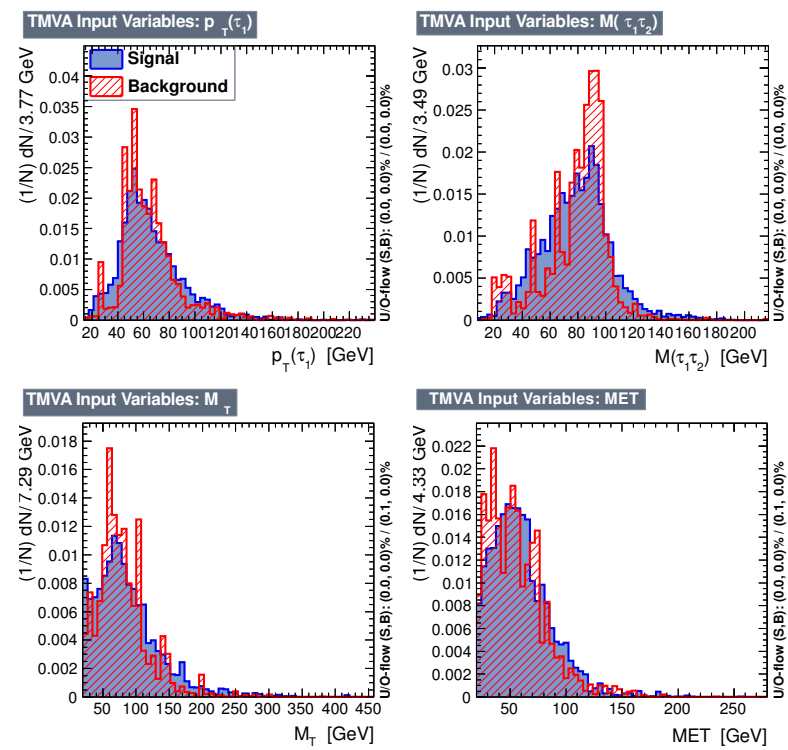

Figure 9.18: The separation between signal and background distributions for the four variables used to train the BDT Pass 2 for a Higgs boson mass of $M_{H}=$ $125 \mathrm{GeV}$. For $p_{T}\left(\tau_{1}\right)$ (top right), $M\left(\tau_{1} \tau_{2}\right)$ (top left), $M_{T}$ (bottom right), and $E_{\mathrm{T}}$ (bottom left).

\section{BDT Pass 2 final discriminant distributions}

The BDT Pass 2 final discriminant distributions is shown in Figure 9.21, showing the background events peaking towards low values and the signal events towards high values. The BDT final discriminant distributions for all mass points studied are available in Appendix A.

\subsection{Systematic uncertainties}

The sources of systematic uncertainties specific to this analysis are described in this section and summarized in Table 9.9. The sources of systematic uncertainties that are general to both analyses covered in this thesis are covered in Section 7.10.

- The uncertainty for the muon identification depends on the quality requirements used, as described in Section 7.10. 

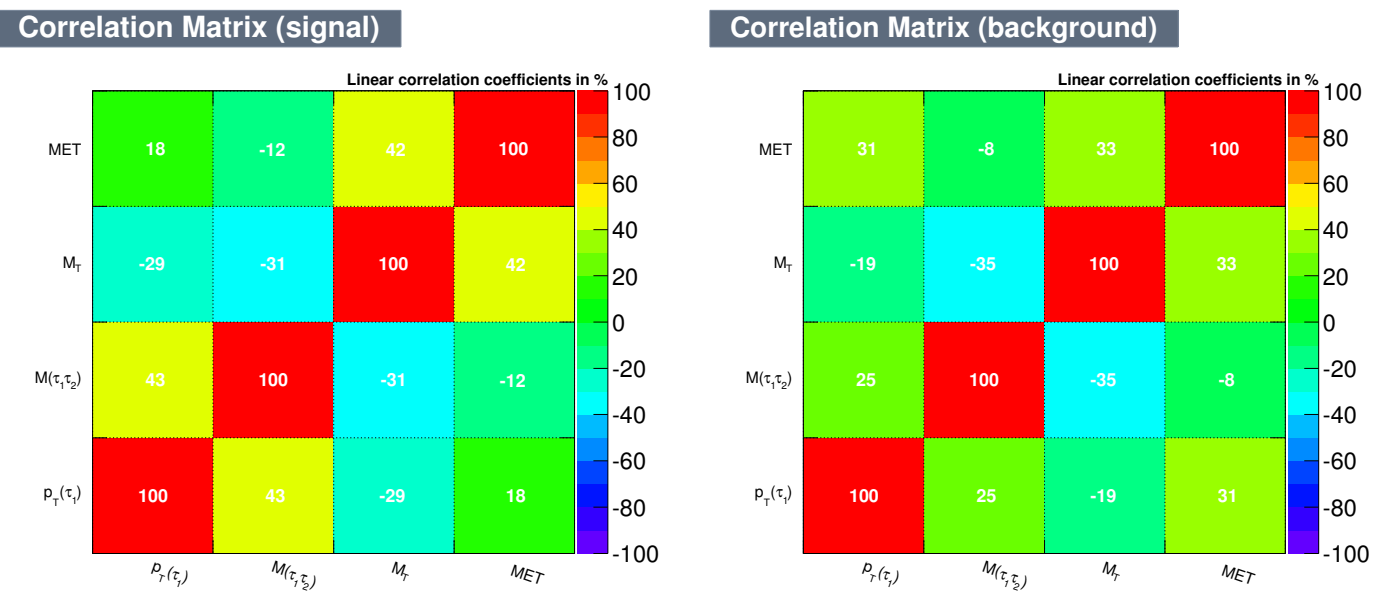

Figure 9.19: The correlation matrices for the BDT Pass 2 for a $125 \mathrm{GeV}$ Higgs boson, for signal (left) and background (right) showing the correlation between the input training variables used, $p_{T}\left(\tau_{1}\right), M\left(\tau_{1} \tau_{2}\right), M_{T}$, and $E_{\mathrm{T}}$.

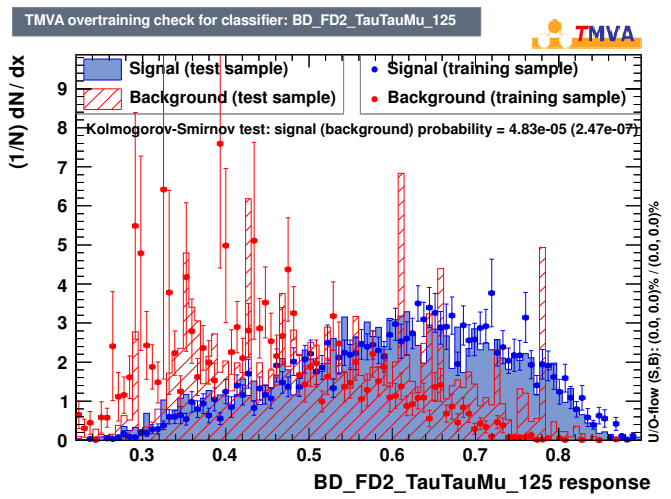

Figure 9.20: The overtraining distribution for the BDT Pass 2 trained for $M_{H}=$ $125 \mathrm{GeV}$. Showing the both signal (red) and the background (blue), for both the training sample (dots) and testing samples (filled histogram). 


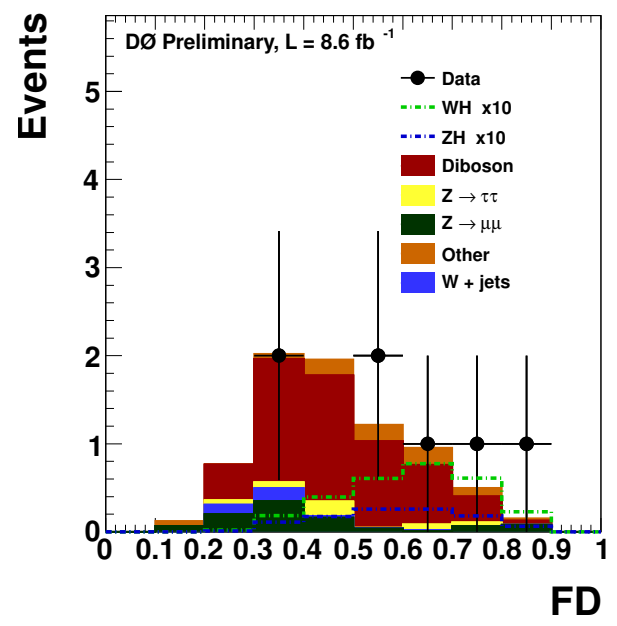

Figure 9.21: The comparison of data to the predicted background events for BDT Pass 2 final discriminant distribution for a $125 \mathrm{GeV}$ Higgs boson. The MC simulation of the associated production of a SM Higgs boson with $M_{H}=125 \mathrm{GeV}$, for the $Z H$ and $W H$ channels, multiplied by a factor of 10 , are superimposed. The 'Other' background sample contains the contribution from $Z \rightarrow e e$ and $t \bar{t}$ events.

- For the "MediumNseg3" muon identification quality in the muon system the determined uncertainty is $1.2 \%$. There are $0.5 \%$ of selected events which have a muon $p_{T}$ of less than $20 \mathrm{GeV}$ which have an additional $2 \%$ error. Leading to an total uncertainty of $1.3 \%$.

- For the "TrackMedium" track quality there is a $1.1 \%$ uncertainty.

- For the "TopScaledMedium" isolation requirements, the uncertainties is $0.5 \%$. In addition $0.5 \%$ of selected events have a muon $p_{T}$ of less than $20 \mathrm{GeV}$, which have an additional $2 \%$ error, this leads to a total uncertainty of $0.6 \%$.

The total muon identification uncertainty is the above three uncertainties combined in quadrature at $1.8 \%$.

- The uncertainty on the efficiency of the $N N_{\tau}$ is shown in Table 9.8 for the 
different data taking epochs. It is dependent on the $N N_{\tau}$ used and the requirement placed on it. The values shown are for the $N N_{2012}$ and the final selection $N N$ requirements, of greater than $0.75 / 0.75 / 0.95$ for Type1/Type-2/Type-3, respectively. The uncertainty of $N N_{\tau}$ requirement is added in quadrature with the uncertainty of the tracking efficiency of $1.4 \%$ as determined from the muon tracking efficiency [101]. As there are at least two tau leptons selected in the final selection and tau lepton types are not differentiated between, a luminosity and tau type weighted average uncertainty of $6.9 \%$ systematic is applied.

\begin{tabular}{|l|cccc|}
\hline tau Type & $N N_{\tau}$ cut & Run IIb1 & Run IIb2 & Run IIb34 \\
\hline Type-1 & $>0.75$ & 9 & 5.5 & 13 \\
Type-2 & $>0.75$ & 4 & 3.5 & 6 \\
Type-3 & $>0.95$ & 6 & 3.5 & 7 \\
\hline
\end{tabular}

Table 9.8: The $N N_{\tau}$ systematics per data epoch, for the $N N_{2012}$ and the selection requirements used, for the three tau types [103], shown in $\%$.

- The uncertainty for the inclusive trigger efficiency is due to shape difference between the final selection sample and the sample the trigger efficiency was determined in. The uncertainty on the inclusive trigger efficiency is determined by varying the multijet contribution in the sample used to determine the efficiency and taking the relative difference in the efficiency as the uncertainty. The uncertainty is determined to be $5 \%$ [109]. This is combined in quadrature with a $5 \%$ uncertainty from the SignalMuonOr trigger uncertainty, as described in Section 7.10, to produce a total uncertainty of $7.1 \%$.

- The uncertainty on the $W+$ jets sample from the $W+$ jets normalization, as described in Section 7.7 is $3 \%$. The uncertainty arises from the statistical uncertainties corresponding to the normalization method and samples used. 


\begin{tabular}{l|ccccccc} 
Contribution & Diboson & $Z / \gamma^{*}$ & $t \bar{t}$ & $W+$ jet & $g g H$ & VBF & $V H$ \\
\hline Luminosity & 6.1 & 6.1 & 6.1 & 6.1 & 6.1 & 6.1 & 6.1 \\
Trigger & 7.1 & 7.1 & 7.1 & 7.1 & 7.1 & 7.1 & 7.1 \\
Cross section & 7.0 & 6.0 & 10.0 & 6.0 & 7.6 & 4.9 & 6.2 \\
$\tau$ lepton Id & 6.9 & 6.9 & 6.9 & 6.9 & 6.9 & 6.9 & 6.9 \\
$W+$ jet & - & - & - & 3.0 & - & - & - \\
reweighting & & & & & & & \\
Muon Id & 1.8 & 1.8 & 1.8 & 1.8 & 1.8 & 1.8 & 1.8
\end{tabular}

Table 9.9: Systematic uncertainties on the signal and background contributions, as described in the Section 7.10 and Section 9.9.

- The cross section uncertainty for the Higgs boson signal samples depends on the factorization and renormalization scales used, as well as on the PDF set used. The scale uncertainties on the factorization and renormalization are determined by varying their value and recalculating the cross section [124] following the prescription of the PDF4LHC group [125]. The PDF uncertainty is taken as determined by the PDF4LHC group [125]. For the separate production samples they have been determined to be [124]:

- Higgs boson associated production, $V H: 6.2 \%$

- Gluon-gluon fusion Higgs boson production, $g g H: 7.6 \%$

- Vector boson fusion Higgs boson production: $4.9 \%$.

\subsection{Cross section limits}

The $95 \%$ C.L. limits on the ratio of the expected and observed cross sections to the predicted SM Higgs boson cross section, are calculated by the COLLIE package [113] (V00-04-12) as described in detail in Section 7.11. All the systematic uncertainties described in Sections 9.9 and 7.10 are included in the calculation. Two input distributions are used by COLLIe to set the limits for all mass points. 
There are the part of the BDT Pass 1 distribution that was not removed by the selection criteria removing the signal and diboson event dominated region and the BDT Pass 2 distribution. Although the BDT Pass 2 distribution contains the majority of the signal events and hence has the greater discriminating power, the signal and background events that did not pass the requirement on BDT Pass 1 still contain significant discriminating power that is utilized in setting limits on the SM Higgs boson cross section.

The distribution of the cross section limits at as a ratio to the predicted SM cross section for the Higgs boson and the corresponding LLR distributions, as a function of the $M_{H}$, are shown in Figure 9.22. The limits are shown for the BDT Pass 2 final discriminant distribution, for the retained region of BDT Pass 1 output distribution and for both distributions combined. The limits are listed in Table 9.10, for the retained region of BDT Pass 1 distribution, Table 9.11 for the BDT Pass 2 distribution and in Table 9.12 for both samples combined.

\begin{tabular}{l|ccccccc}
$M_{H}[\mathrm{GeV}]$ & 100 & 105 & 110 & 115 & 120 & 125 & 130 \\
\hline$\sigma_{\text {exp }} / \sigma_{\text {exp }}(S M)$ & 16.8 & 18.8 & 19.2 & 24.4 & 27.4 & 27.9 & 26.6 \\
$\sigma_{\text {obs }} / \sigma_{\text {obs }}(S M)$ & 28.6 & 17.9 & 27.3 & 29.5 & 28.6 & 36.3 & 33.4 \\
\hline & & & & & & & \\
\hline$M_{H}[\mathrm{GeV}]$ & 135 & 140 & 145 & 150 & 155 & 160 & 165 \\
\hline$\sigma_{\text {exp }} / \sigma_{\text {exp }}(S M)$ & 27.8 & 29.9 & 32.1 & 33.2 & 33.9 & 45.1 & 47.9 \\
$\sigma_{\text {obs }} / \sigma_{\text {obs }}(S M)$ & 28.9 & 35.3 & 37.9 & 37.2 & 36.6 & 59.7 & 51.3 \\
\hline & & & & & & & \\
\hline$M_{H}[\mathrm{GeV}]$ & 170 & 175 & 180 & 185 & 190 & 195 & 200 \\
\hline$\sigma_{\text {exp }} / \sigma_{\text {exp }}(S M)$ & 52.0 & 48.2 & 55.9 & 55.3 & 66.8 & 71.4 & 68.7 \\
$\sigma_{\text {obs }} / \sigma_{\text {obs }}(S M)$ & 50.2 & 59.6 & 64.9 & 64.9 & 68.2 & 67.7 & 99.7
\end{tabular}

Table 9.10: Expected and observed cross section limits at 95\% C.L., given as a ratio to the predicted SM Higgs cross section, per SM Higgs boson mass point studied, for the retained region of BDT Pass 1. 
BDT Pass 1 Limits

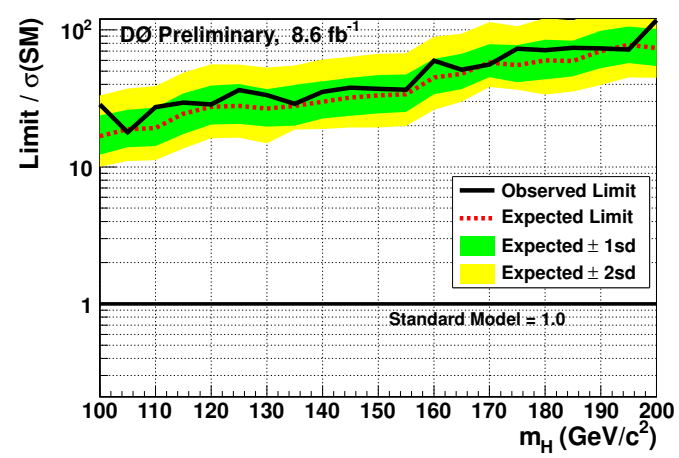

BDT Pass 2 Limits

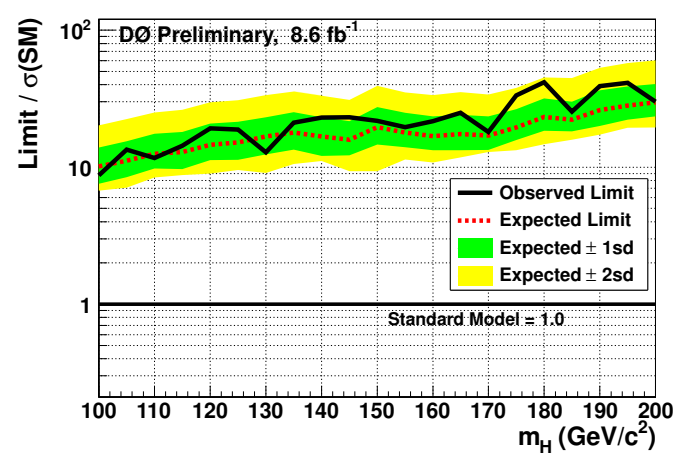

Combined Limits

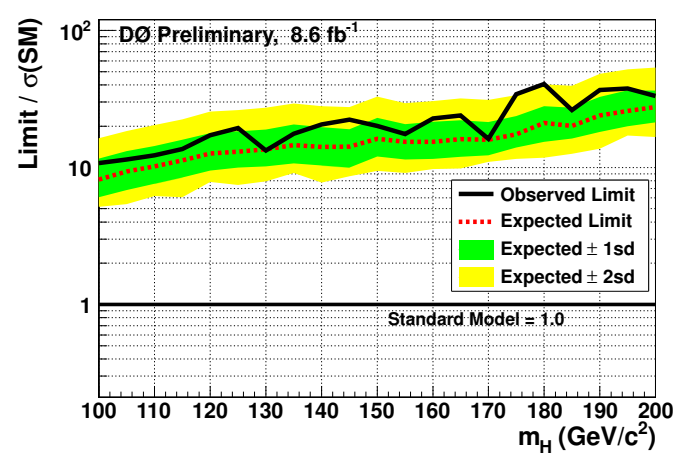

BDT Pass 1 LLR

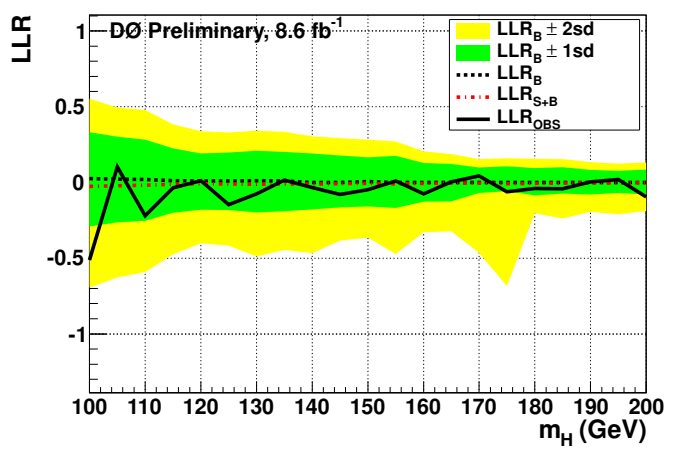

BDT Pass 2 LLR

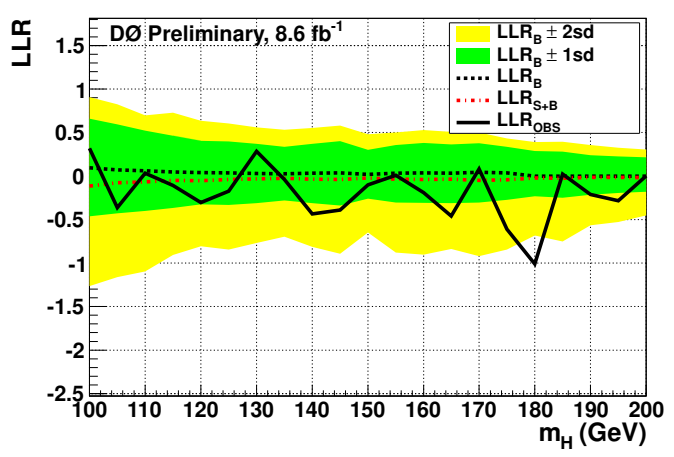

Combined LLR

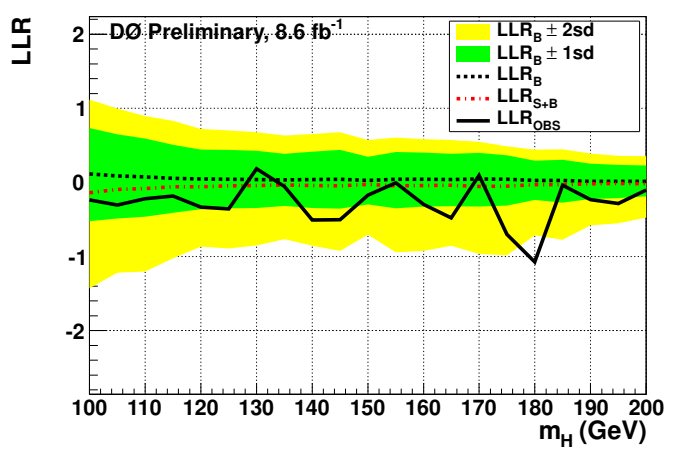

Figure 9.22: The expected and observed cross section limits at 95\% C.L. as a ratio to the SM Higgs boson cross section and the corresponding log likelihood ratio plots. For the retained region of BDT Pass 1 (top), the BDT Pass 2 (middle), and for both combined (bottom). The expected limits are shown in black, the observed in red, and the yellow and green bands correspond to the $\pm 1 \sigma$ and $\pm 2 \sigma$ variation on the expected limits. 


\begin{tabular}{l|ccccccc}
$M_{H}[\mathrm{GeV}]$ & 100 & 105 & 110 & 115 & 120 & 125 & 130 \\
\hline$\sigma_{\text {exp }} / \sigma_{\text {exp }}(S M)$ & 10.1 & 11.1 & 12.5 & 12.9 & 14.6 & 15.1 & 16.8 \\
$\sigma_{\text {obs }} / \sigma_{\text {obs }}(S M)$ & 8.7 & 13.4 & 11.7 & 14.4 & 19.2 & 18.8 & 12.8 \\
\hline & & & & & & & \\
\hline$M_{H}[\mathrm{GeV}]$ & 135 & 140 & 145 & 150 & 155 & 160 & 165 \\
\hline$\sigma_{\text {exp }} / \sigma_{\text {exp }}(S M)$ & 17.9 & 16.8 & 15.9 & 19.6 & 17.9 & 16.8 & 17.5 \\
$\sigma_{\text {obs }} / \sigma_{\text {obs }}(S M)$ & 21.2 & 23.0 & 23.2 & 21.8 & 19.6 & 21.6 & 24.9 \\
\hline & & & & & & & \\
\hline$M_{H}[\mathrm{GeV}]$ & 170 & 175 & 180 & 185 & 190 & 195 & 200 \\
\hline$\sigma_{\text {exp }} / \sigma_{\text {exp }}(S M)$ & 17.0 & 19.5 & 23.3 & 22.2 & 26.1 & 28.2 & 29.8 \\
$\sigma_{\text {obs }} / \sigma_{\text {obs }}(S M)$ & 18.1 & 33.5 & 41.7 & 25.4 & 39.1 & 41.4 & 30.11
\end{tabular}

Table 9.11: Expected and observed cross section limits as a ratio of the predicted SM Higgs cross section, at 95\% C.L. per SM Higgs boson mass point studied, for BDT Pass 2.

\begin{tabular}{l|ccccccc}
$M_{H}[\mathrm{GeV}]$ & 100 & 105 & 110 & 115 & 120 & 125 & 130 \\
\hline$\sigma_{\text {exp }} / \sigma_{\text {exp }}(S M)$ & 8.2 & 9.3 & 10.2 & 11.3 & 12.7 & 13.0 & 13.5 \\
$\sigma_{\text {obs }} / \sigma_{\text {obs }}(S M)$ & 10.8 & 11.4 & 12.3 & 13.6 & 17.2 & 19.4 & 13.3 \\
\hline & & & & & & & \\
\hline$M_{H}[\mathrm{GeV}]$ & 135 & 140 & 145 & 150 & 155 & 160 & 165 \\
\hline$\sigma_{\text {exp }} / \sigma_{\text {exp }}(S M)$ & 14.6 & 14.1 & 14.2 & 16.2 & 15.4 & 15.4 & 16.1 \\
$\sigma_{\text {obs }} / \sigma_{\text {obs }}(S M)$ & 17.6 & 20.6 & 22.3 & 20.1 & 17.6 & 22.8 & 23.9 \\
\hline & & & & & & & \\
\hline$M_{H}[\mathrm{GeV}]$ & 170 & 175 & 180 & 185 & 190 & 195 & 200 \\
\hline$\sigma_{\text {exp }} / \sigma_{\text {exp }}(S M)$ & 16.0 & 17.4 & 21.1 & 20.1 & 24.0 & 25.9 & 27.5 \\
$\sigma_{\text {obs }} / \sigma_{\text {obs }}(S M)$ & 16.2 & 34.3 & 40.7 & 26.2 & 36.7 & 37.8 & 33.3
\end{tabular}

Table 9.12: Expected and observed cross section limits as a ratio of the predicted SM Higgs cross section, at 95\% C.L. per SM Higgs boson mass point studied, for the combined sample of both the BDT Pass 2 and the retained region of BDT Pass 1. 


\section{Chapter 10}

\section{Conclusion}

In this thesis, the Higgs boson of the Standard Model (SM) and Higgs bosons predicted by theories beyond the SM are studied in final states with multiple tau leptons, using data recorded with the $\mathrm{D} \varnothing$ detector at the Fermilab Tevatron.

Doubly charged Higgs bosons, predicted by theories beyond the Standard Model (SM), such as Little Higgs models and Left-Right Symmetric models, are expected to decay into like-charge lepton pairs. A search for the existence of pair produced doubly charged Higgs bosons, based on $7.0 \mathrm{fb}^{-1}$ of integrated luminosity, is used to set limits on five benchmark points: (1) $\mathcal{B}\left(H^{ \pm \pm} \rightarrow \tau \tau\right)=1$, (2) $\mathcal{B}\left(H^{ \pm \pm} \rightarrow \mu \mu\right)=1,(3) \mathcal{B}\left(H^{ \pm \pm} \rightarrow \mu \tau\right)=1,(4) \mathcal{B}\left(H^{ \pm \pm} \rightarrow \mu \mu\right)+\mathcal{B}\left(H^{ \pm \pm} \rightarrow\right.$ $\tau \tau)=1$ and $(5) \mathcal{B}\left(H^{ \pm \pm} \rightarrow \tau \tau\right)=\mathcal{B}\left(H^{ \pm \pm} \rightarrow \mu \mu\right)=\mathcal{B}\left(H^{ \pm \pm} \rightarrow \mu \tau\right)=1 / 3$. Cross section limits are set for both left and right handed $H^{ \pm \pm}$bosons, except for Point (4), where right handed $H^{ \pm \pm}$bosons do not exist. The highest sensitivity is reached for Point (3) with $\mathcal{B}\left(H^{ \pm \pm} \rightarrow \mu \mu\right)=1$ where an expected (observed) lower limit on the the mass of $M\left(H^{ \pm \pm}\right)>180 \mathrm{GeV}(168 \mathrm{GeV})$ is found for $H_{\mathrm{L}}^{ \pm \pm}$ states and $M\left(H^{ \pm \pm}\right)>154 \mathrm{GeV}(145 \mathrm{GeV})$ for $H_{\mathrm{R}}^{ \pm \pm}$states. The efficiency for the reconstruction of tau leptons is lower than for muons, leading to limits for Point (1) of $M\left(H_{\mathrm{L}}^{ \pm \pm}\right)>116 \mathrm{GeV}(128 \mathrm{GeV})$. The limits for right-handed states in tau final states lie below the previous LEP limit and are therefore not considered. 
The limits for the other benchmark points, with mixed tau lepton and muon final states, lie between the values for Points (1) and (2).

This search set the world's best published limits for these $H^{ \pm \pm}$decay channels and is the first search performed for Points (1), (4) and (5) at a hadron collider. Since publication, these results have been superseded by results from the LHC. The CMS experiment studies pair of singly produced doubly charged Higgs bosons decaying to $\ell \ell^{\prime}$ pairs where $\ell$ and $\ell^{\prime}$ can be $e, \mu$, or $\tau$. The resulting limits are in the range $M\left(H_{\mathrm{L}}^{ \pm \pm}\right)>165-457 \mathrm{GeV}$, for the different production and decay modes of the $H^{ \pm \pm}$boson studied [40]. The ATLAS experiment has published limit for the $\mathcal{B}\left(H^{ \pm \pm} \rightarrow \mu \mu\right)=1$ channel, setting limits of $M\left(H_{\mathrm{L}}^{ \pm \pm}\right)>355 \mathrm{GeV}$ and $M\left(H_{\mathrm{R}}^{ \pm \pm}\right)>251 \mathrm{GeV}[41]$.

The Higgs boson of the SM is studied at the Tevatron through a large number of channels. Some of these channels lead to multiple tau leptons in the final state, either from decays of the Higgs boson or from decays of $W$ and $Z$ bosons. A search for the SM Higgs boson in such final states with multiple tau leptons, $\tau \tau \mu+X$, is performed. This analysis utilises an integrated luminosity of $8.6 \mathrm{fb}^{-1}$ and makes extensive use of multivariate techniques to improve the sensitivity to a SM Higgs signal. Limits are set on the ratio of the signal cross section to the theoretical cross section for a SM Higgs boson. The expected (observed) limit on this ratio for a SM Higgs boson with a mass of $M_{H}=125 \mathrm{GeV}$ is determined to be 12.7 (17.2).

This represents the first search in this final state using DØ data. A fermionic decay channel is explored, where no evidence for SM Higgs boson has yet been observed. The sensitivity of this search alone is not sufficient to confirm the SM Higgs boson-like excess as seen by the CMS and ATLAS experiments [31, 32]. However, it provides an important input to the combination of search channels at the Tevatron, adding sensitivity at $M_{H}<140 \mathrm{GeV}$. The combined Tevatron 
data has provided first evidence for Higgs production in associated production with $W$ and $Z$ bosons [2].

In addition to the Higgs boson searches using D $\varnothing$ data, the implementation of tree level couplings of the next-to-minimal supersymmetric Standard Model (NMSSM) into the HERWIG++ MC generator is documented. All Feynman rules associated with the tree level couplings in the NMSSM, as well as the loop induced coupling of the Higgs bosons to a pair of gluons or photons are added. The implemented couplings are validated by comparison to NMHDECAY decay and within HERWIG ++ in the limit where the NMSSM collapses to the MSSM. They are determined to accurately reproduce the results for all decays and over a selection of benchmark points that cover a wide range of the parameter space within the NMSSM. Results from the LHC experiments do not invalidate the NMSSM, and the model can incorporate an enhanced $H \rightarrow \gamma \gamma$ coupling to explain the higher than predicted rate of $H \rightarrow \gamma \gamma$ decays that is observed at the LHC [126]. The NMSSM therefore remains an important field of study, and these studies will be facilitated by the implementation of the NMSSM into the HERWIG++ generator. 


\section{Appendix A}

\section{BDT outputs}

The determined BDT outputs showing the observed data compared to the predicted MC events are shown for both BDT Pass 1 (Figures A.1 and A.2) and BDT Pass 2 (Figures A.3 and A.4). The output distributions are given for all masses studied, 100 - $200 \mathrm{GeV}$, in $5 \mathrm{GeV}$ intervals and are shown with the predicted signal for the associated production of a SM Higgs boson with a $W$ or $Z$ boson of the corresponding mass, multiplied by a factor of 10 superimposed. 
$100 \mathrm{GeV}$

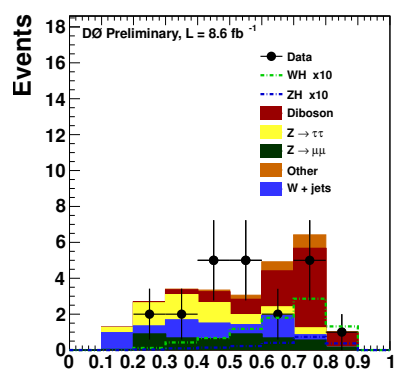

FD

$115 \mathrm{GeV}$

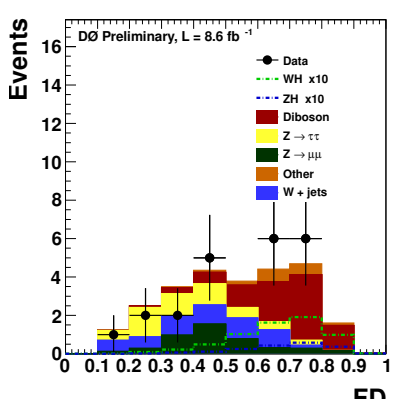

$130 \mathrm{GeV}$

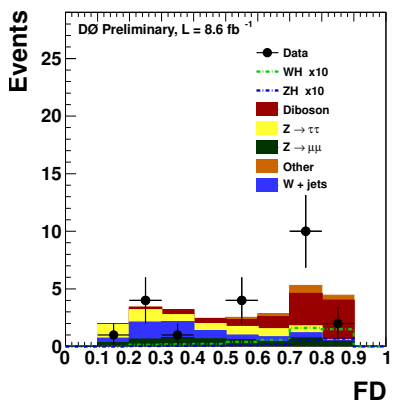

$105 \mathrm{GeV}$

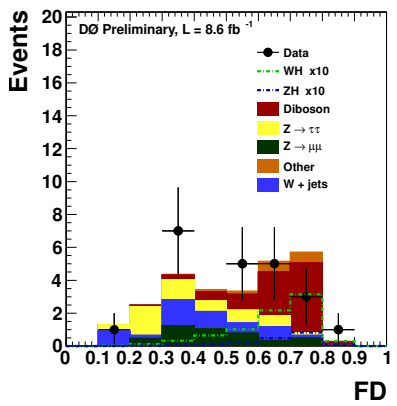

$120 \mathrm{GeV}$

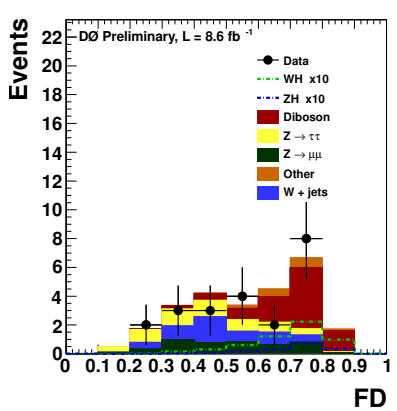

$135 \mathrm{GeV}$

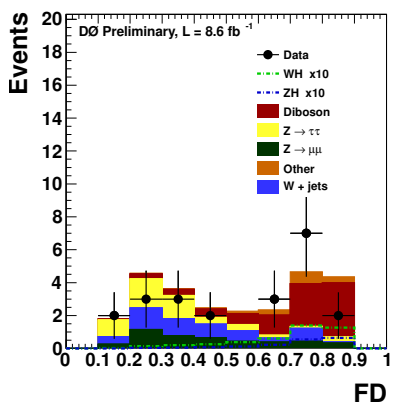

$110 \mathrm{GeV}$

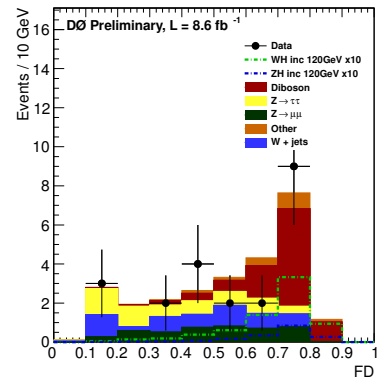

$125 \mathrm{GeV}$

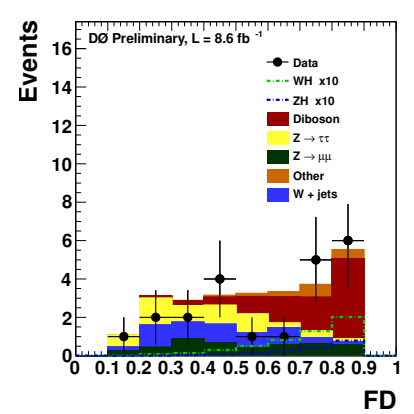

$140 \mathrm{GeV}$

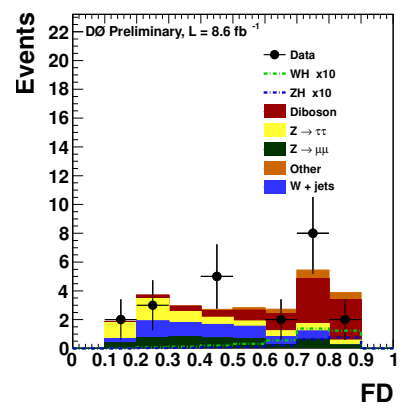

Figure A.1: The BDT Pass 1 discriminant distribution, for the observed data compared to predicted MC simulation backgrounds. The signal sample for associated production of a SM Higgs boson, multiplied by a factor of 10 is superimposed. 
$145 \mathrm{GeV}$

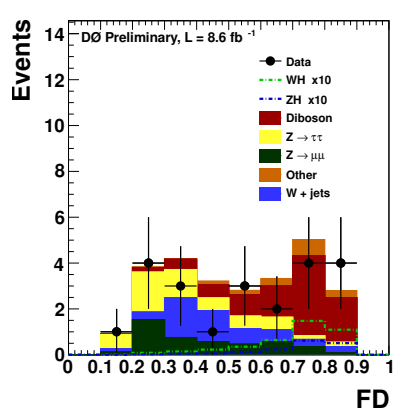

$160 \mathrm{GeV}$

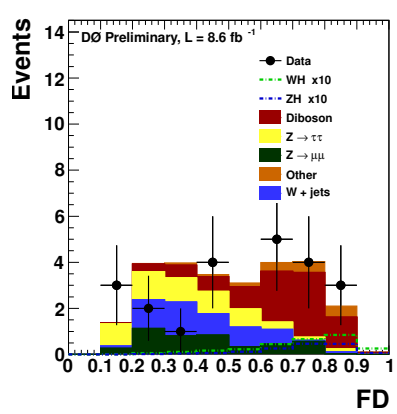

$175 \mathrm{GeV}$

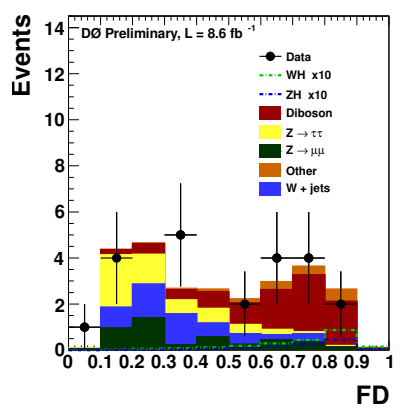

$190 \mathrm{GeV}$

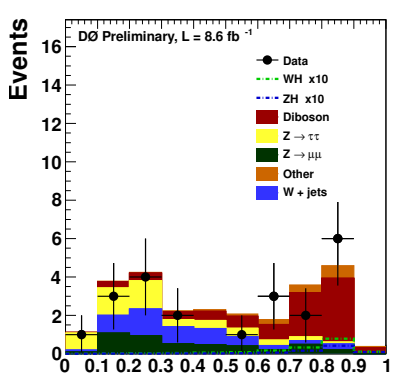

FD
$150 \mathrm{GeV}$

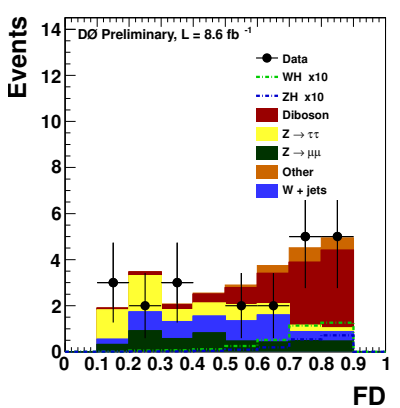

$165 \mathrm{GeV}$

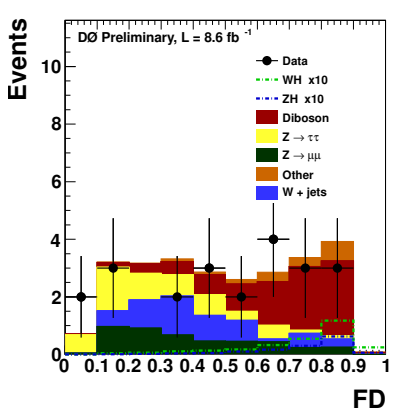

$180 \mathrm{GeV}$

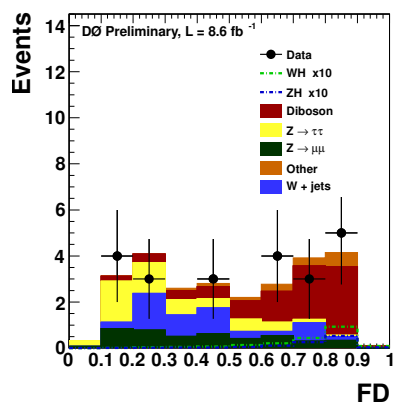

$195 \mathrm{GeV}$

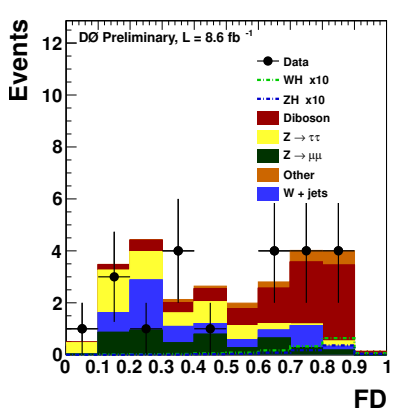

$155 \mathrm{GeV}$

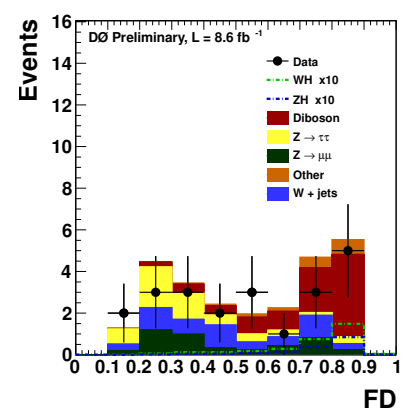

$170 \mathrm{GeV}$

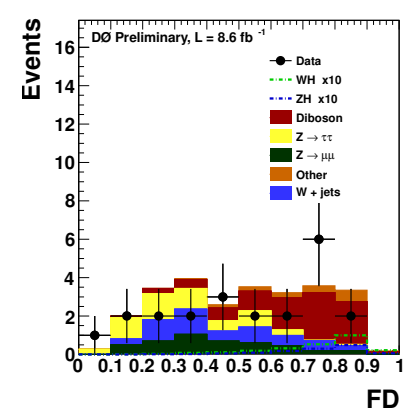

$185 \mathrm{GeV}$

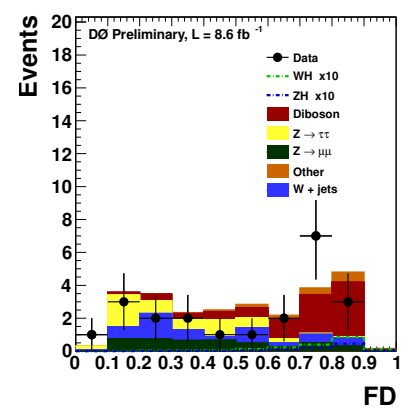

$200 \mathrm{GeV}$

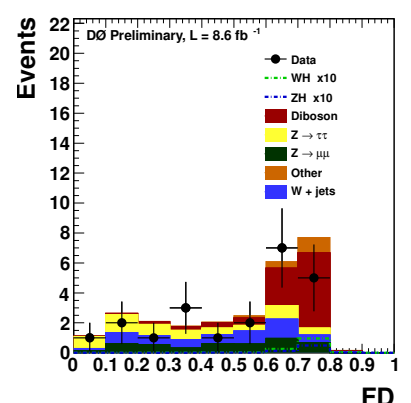

Figure A.2: The BDT Pass 1 discriminant distribution, for the observed data compared to predicted MC simulation backgrounds. The signal sample for associated production of a SM Higgs boson, multiplied by a factor of 10 is superimposed. 
$100 \mathrm{GeV}$

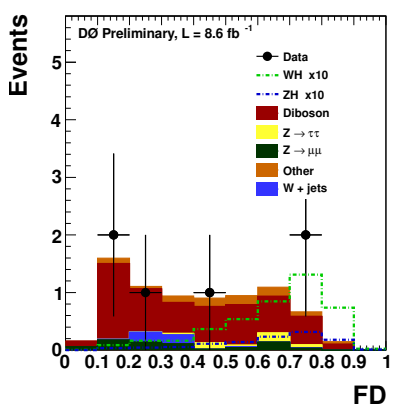

$115 \mathrm{GeV}$

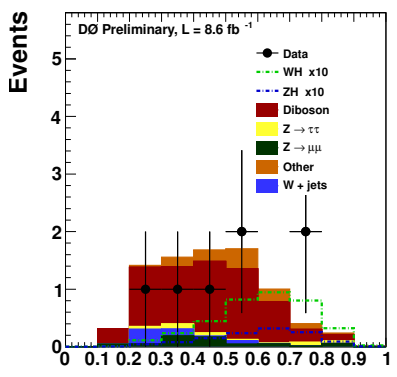

FD

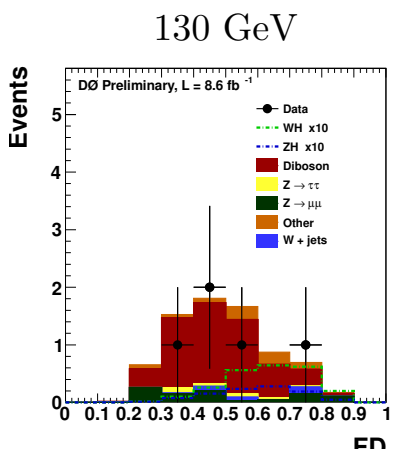

$105 \mathrm{GeV}$

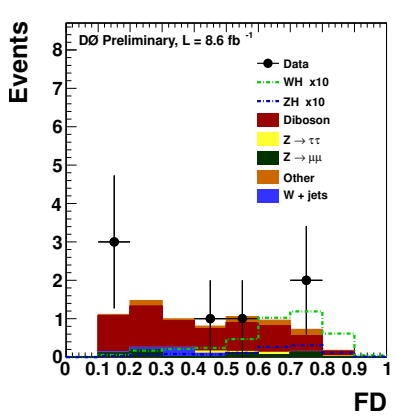

$120 \mathrm{GeV}$

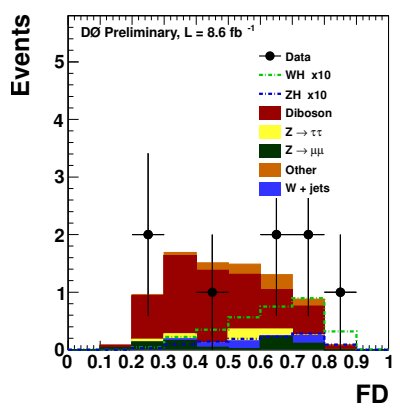

$135 \mathrm{GeV}$

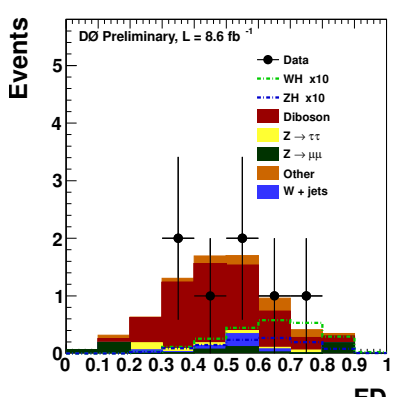

$110 \mathrm{GeV}$

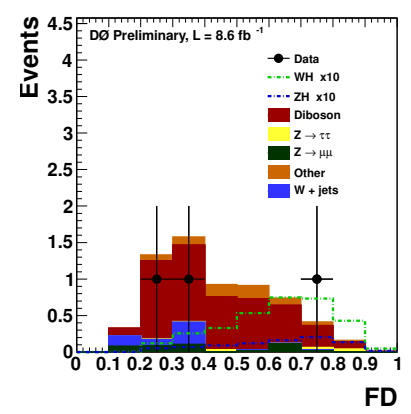

$125 \mathrm{GeV}$

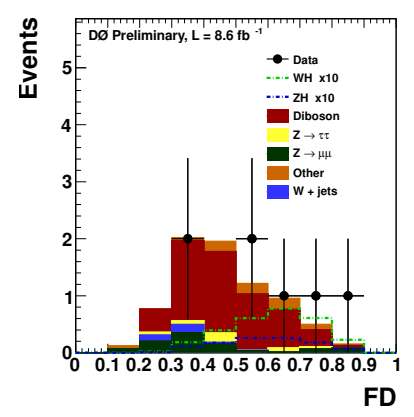

$140 \mathrm{GeV}$

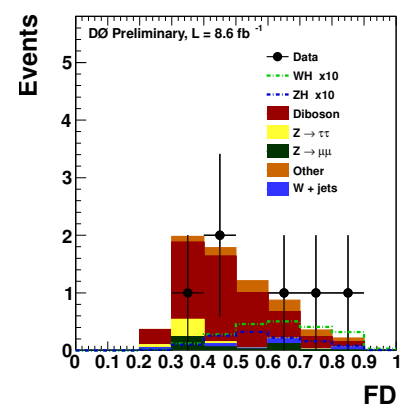

Figure A.3: The BDT Pass 2 discriminant distribution, for the observed data compared to predicted MC simulation backgrounds. The signal sample for associated production of a SM Higgs boson, multiplied by a factor of 10 is superimposed. 
$145 \mathrm{GeV}$

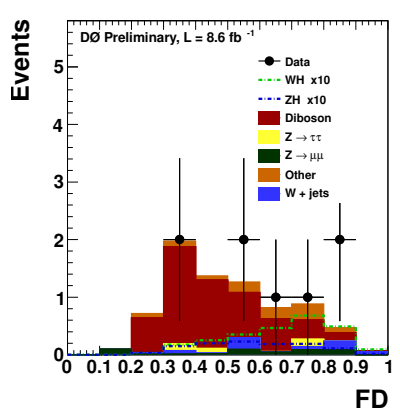

$160 \mathrm{GeV}$

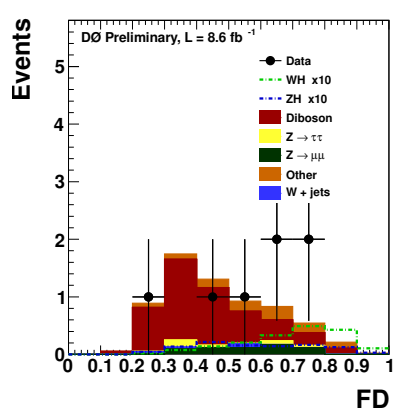

$175 \mathrm{GeV}$

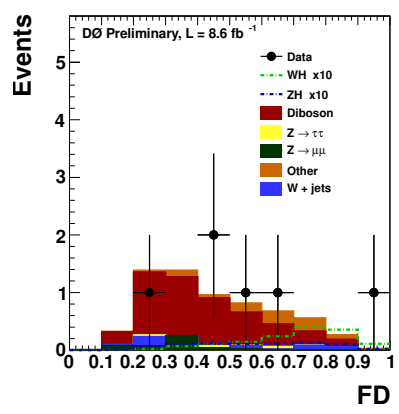

$190 \mathrm{GeV}$

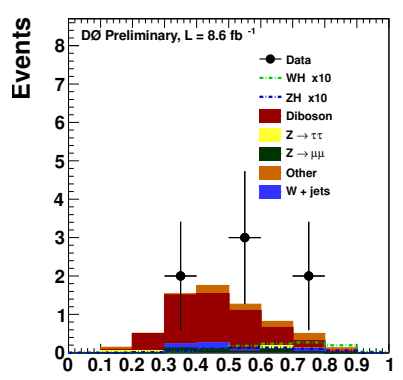

FD
$150 \mathrm{GeV}$

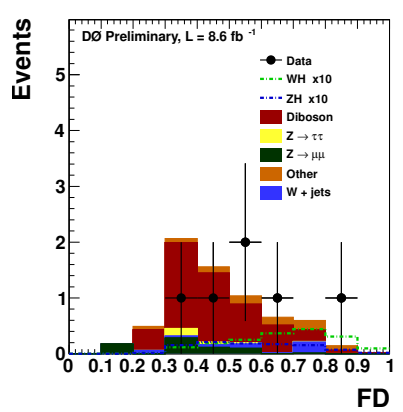

$165 \mathrm{GeV}$

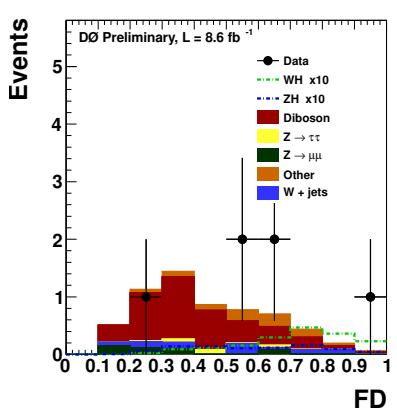

$180 \mathrm{GeV}$

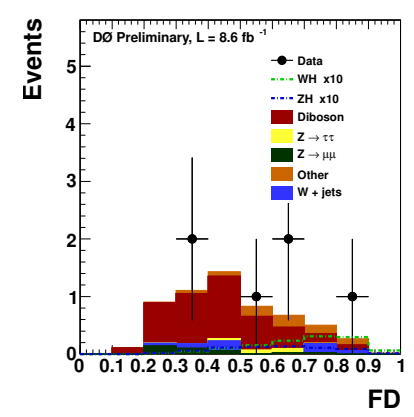

$195 \mathrm{GeV}$

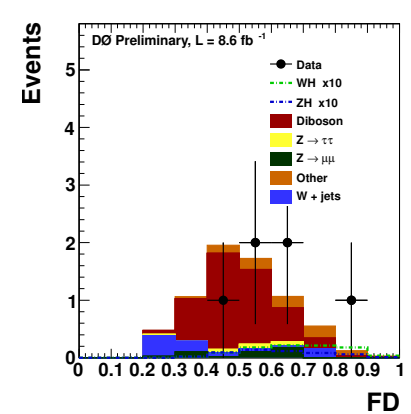

$155 \mathrm{GeV}$

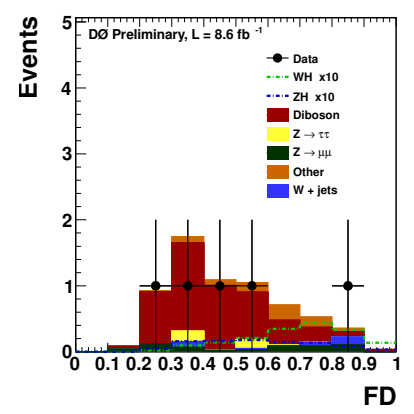

$170 \mathrm{GeV}$

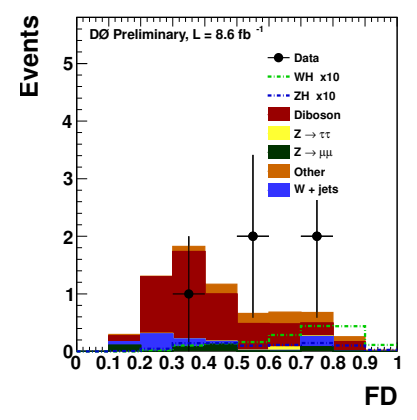

$185 \mathrm{GeV}$

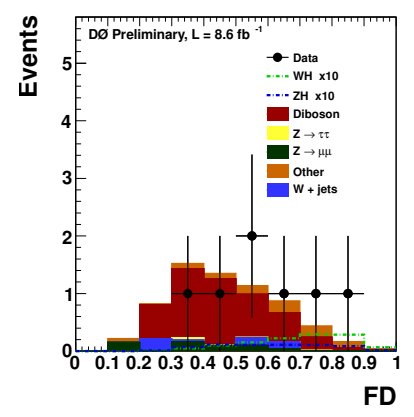

$200 \mathrm{GeV}$

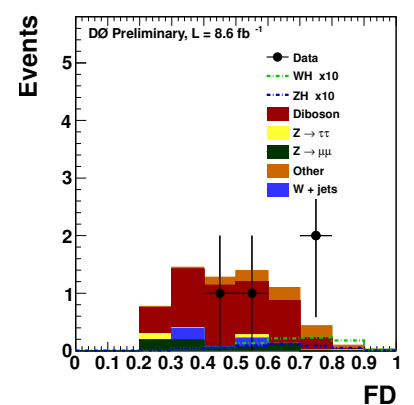

Figure A.4: The BDT Pass 2 discriminant distribution, for the observed data compared to predicted MC simulation backgrounds. The signal sample for associated production of a SM Higgs boson, multiplied by a factor of 10 is superimposed. 


\section{Appendix B}

\section{The NMSSM Feynman Rules}

The Feynman rules as used by Herwig ++ are given below $[7,127]$. The following parameters are used; the mixing matrices for the scalar Higgs boson, $S_{i j}$, the pseudoscalar Higgs boson, $P_{i j}$, the chargions, $U_{i j}$ and $V_{i j}$, and for the neutralino, $N_{i j}$. The weak and electromagnetic coupling constants are given by $g, g^{\prime}$ respectively. $\theta_{W}$ is the Weinberg angle, $p$ is the momentum, $s, h_{u}, h_{d}$ are the VEV's of the singlet Higgs boson and up-type and down-type Higgs boson doublets respectively, $e_{u, d}$ are the charges of the up-type and down-type quarks respectively, both defined as positive, $A_{U, D}$ are the up-type and down-type trilinear couplings. In the following $H_{i}$ stands for $h_{1}, h_{2}$ and $h_{3}$ and $A_{i}$ stands for $a_{1}$ and $a_{2}$.

\section{B.1 The Higgs boson-fermion vertices}

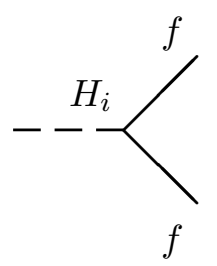

$$
\begin{array}{lc}
\text { u-type } & \text { d-type } \\
\frac{g m_{u}}{2 m_{W} \sin \beta} S_{i 2} & \frac{g m_{d}}{2 m_{W} \cos \beta} S_{i 1}
\end{array}
$$



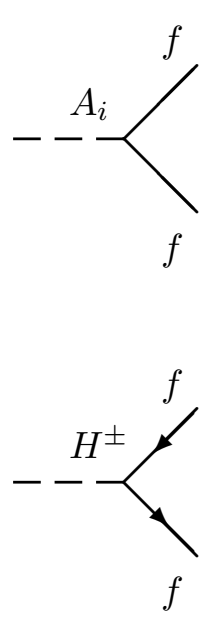

$$
\text { u-type }
$$$$
\frac{i g m_{u}}{2 m_{W} \sin \beta} P_{i 2}
$$$$
\mathbf{H}^{+} \mathbf{d u}
$$$$
-\frac{g m_{u}}{\sqrt{2} m_{W}} \cot \beta
$$

d-type

\section{B.2 Higgs boson-gauge bosons vertices}

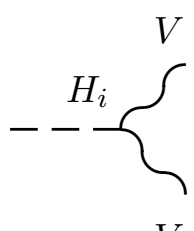

HZZ

HWW

V

$\frac{g m_{W}}{\cos ^{2} \theta_{W}}\left(\cos \beta S_{i 1}+\sin \beta S_{i 2}\right) \quad g m_{W}\left(\cos \beta S_{i 1}+\sin \beta S_{i 2}\right)$

\section{B.2.1 Double Higgs boson-gauge boson vertices}

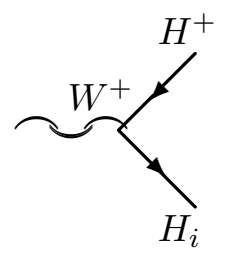

$$
\frac{g}{2}\left(\cos \beta S_{i 2}-\sin \beta S_{i 1}\right)\left(p-p^{\prime}\right)_{\mu}
$$




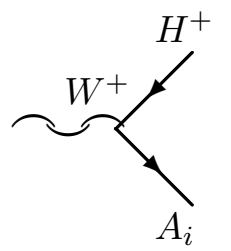

$$
i \frac{g}{2}\left(\cos \beta P_{i 2}+\sin \beta P_{i 1}\right)\left(p-p^{\prime}\right)_{\mu}
$$

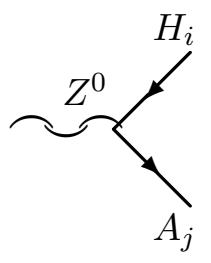

$$
i \frac{g}{2 \cos \theta_{W}}\left(S_{i 2} P_{j 2}-S_{i 1} P_{j 1}\right)\left(p-p^{\prime}\right)_{\mu}
$$

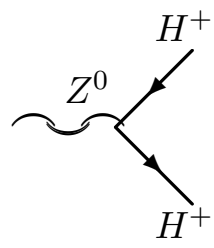

$$
\frac{g\left(\cos ^{2} \theta_{W}-\sin ^{2} \theta_{W}\right)}{\cos \theta_{W}}\left(p-p^{\prime}\right)_{\mu}
$$

\section{B.2.2 Higgs boson-gauginos vertices}

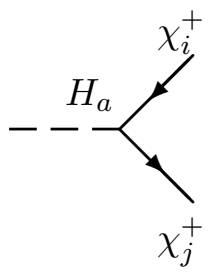

$$
\frac{\lambda}{\sqrt{2}} S_{a 3} U_{i 2} V_{j 2}+\frac{g}{\sqrt{2}}\left(S_{a 2} U_{i 1} V_{j 2}+S_{a 1} U_{i 2} V_{j 1}\right)
$$

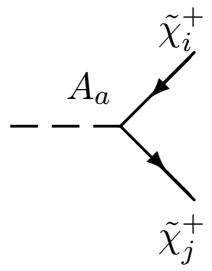

$$
i\left(\frac{\lambda}{\sqrt{2}} P_{a 3} U_{i 2} V_{j 2}-\frac{g}{\sqrt{2}}\left(P_{a 2} U_{i 1} V_{j 2}+P_{a 1} U_{i 2} V_{j 1}\right)\right)
$$

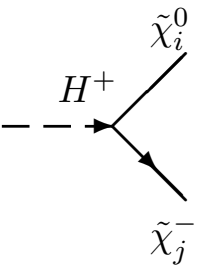

$$
\lambda \cos \beta U_{i 2} N_{j 5}-\frac{\sin \beta}{\sqrt{2}} U_{i 2}\left(g^{\prime} N_{j 1}+g N_{i 2}\right)+g \sin \beta U_{i 1} N_{j 3}
$$




$$
\begin{aligned}
& \chi_{j}^{+} \\
& \chi_{j}^{0} \sin \beta V_{i 2} N_{j 5}+\frac{\cos \beta}{\sqrt{2}} V_{i 2}\left(g^{\prime} N_{j 1}+g N_{i 2}\right)+g \cos \beta V_{i 1} N_{j 4} \\
& \frac{\lambda}{\sqrt{2}} S_{a 2} \Pi_{i j}^{45}+S_{a 1} \Pi_{i j}^{35}+S_{a 3} \Pi_{i j}^{34}-\sqrt{2} \kappa S_{a 3} N_{i 5} N_{j 5} \\
& +\frac{g^{\prime}}{2}\left(S_{a 2} \Pi_{i j}^{13}-S_{a 1} \Pi_{i j}^{14}\right)+\frac{g}{2}\left(S_{a 2} \Pi_{i j}^{23}-S_{a 1} \Pi_{i j}^{24}\right) \\
& \chi_{j}^{0} \\
& \chi_{j}^{0} \\
& \chi_{j}^{0} \\
& \left.-\frac{g^{\prime}}{2}\left(P_{a 1} \Pi_{i j}^{13}-P_{a 2} \Pi_{i j}^{14}\right)+\frac{g}{2}\left(P_{a 1} \Pi_{i j}^{23}-P_{a 1} \Pi_{i j}^{24}\right)\right) \\
& A_{a 1}^{45} \Pi_{i j}^{45}+P_{a 2} \Pi_{i j}^{35}+P_{a 3} \Pi_{i j}^{34}+\sqrt{2} \kappa P_{a 3} N_{i 5} N_{j 5} \\
& \Pi_{i j}=N_{i a} N_{j b}+N_{i b} N_{j a}
\end{aligned}
$$

\section{B.2.3 Triple Higgs boson vertices}

$$
\begin{gathered}
H_{b} \quad \begin{array}{c}
\frac{\lambda^{2}}{\sqrt{2}}\left(h_{u}\left(\Pi_{a b c}^{211}+\Pi_{a b c}^{233}\right)+h_{d}\left(\Pi_{a b c}^{122}+\Pi_{a b c}^{133}\right)+s\left(\Pi_{a b c}^{322}+\Pi_{a b c}^{311}\right)\right) \\
-\frac{\lambda \kappa}{\sqrt{2}}\left(h_{u} \Pi_{a b c}^{133}+h_{d} \Pi_{a b c}^{323}+2 s \Pi_{a b c}^{213}\right)+\sqrt{2} \kappa^{2} s \Pi_{a b c}^{333} \\
-\frac{\lambda A_{\lambda}}{\sqrt{2}} \Pi_{a b c}^{213}+\frac{\kappa A_{\kappa}}{3 \sqrt{2}} \Pi_{a b c}^{333} \\
+\frac{\left(g^{2}+g^{\prime 2)}\right.}{4 \sqrt{2}}\left(h_{u}\left(\Pi_{a b c}^{222}-\Pi_{a b c}^{211}\right)-h_{d}\left(\Pi_{a b c}^{122}-\Pi_{a b c}^{111}\right)\right) \\
\Pi_{a b c}^{i j k}=S_{a i} S_{b j} S_{c k}+S_{a i} S_{c j} S_{b k}+S_{b i} S_{a j} S_{c k} \\
S_{b j} S_{c j} S_{a k}+S_{c i} S_{a j} S_{b k}+S_{c i} S_{b j} S_{a k}
\end{array}
\end{gathered}
$$




$$
\begin{aligned}
& H_{a} A_{b} \frac{\lambda^{2}}{\sqrt{2}}\left(h_{u}\left(\Pi_{a b c}^{211}+\Pi_{a b c}^{233}\right)+h_{d}\left(\Pi_{a b c}^{122}+\Pi_{a b c}^{133}\right)+s\left(\Pi_{a b c}^{322}+\Pi_{a b c}^{311}\right)\right) \\
& +\frac{\lambda \kappa}{\sqrt{2}}\left(h_{u}\left(\Pi_{a b c}^{133}-2 \Pi_{a b c}^{313}\right)+h_{d}\left(\Pi_{a b c}^{233}-2 \Pi_{a b c}^{323}\right)\right. \\
& \left.+2 s\left(\Pi_{a b c}^{321}-\Pi_{a b c}^{213}-\Pi_{a b c}^{123}\right)\right)+\sqrt{2} \kappa^{2} s \Pi_{a b c}^{333} \\
& +\frac{\lambda A_{\lambda}}{\sqrt{2}}\left(\Pi_{a b c}^{213}+\Pi_{a b c}^{123}+\Pi_{a b c}^{321}\right)-\frac{\kappa A_{\kappa}}{\sqrt{2}} \Pi_{a b c}^{333} \\
& +\frac{\left(g^{2}+g^{\prime 2)}\right.}{4 \sqrt{2}}\left(h_{u}\left(\Pi_{a b c}^{222}-\Pi_{a b c}^{211}\right)-h_{d}\left(\Pi_{a b c}^{122}-\Pi_{a b c}^{111}\right)\right) \\
& \Pi_{a b c}^{i j k}=S_{a i}\left(P_{b j} P_{c k}+P_{c j} P_{b k}\right) \\
& \frac{\lambda^{2}}{\sqrt{2}}\left(s\left(\Pi_{a}^{322}+\Pi_{a}^{311}\right)-h_{u} \Pi_{a}^{121}-h_{d} \Pi_{a}^{221}\right) \\
& +\sqrt{2} \lambda \kappa s \Pi_{a}^{321}+\frac{\lambda A_{\lambda}}{\sqrt{2}} \Pi_{a}^{321} \\
& +\frac{g^{\prime 2}}{4 \sqrt{2}}\left(h_{u}\left(\Pi_{a}^{222}-\Pi_{a}^{211}\right)+h_{d}\left(\Pi_{a}^{111}-\Pi_{a}^{122}\right)\right. \\
& +\frac{g^{2}}{4 \sqrt{2}}\left(h _ { u } \left(\Pi_{a}^{222}+\Pi_{a}^{211}\right.\right. \\
& \left.\left.+2 \Pi_{a}^{121}\right)+h_{d}\left(\Pi_{a}^{122}+\Pi_{a}^{111}+2 \Pi_{a}^{221}\right)\right) \\
& \Pi_{a}^{i j k}=2 S_{a i} C_{J} C_{k} \\
& C_{1}=\cos \beta, C_{2}=\sin \beta
\end{aligned}
$$

\section{B.2.4 Scalar fermion-Higgs boson vertices}

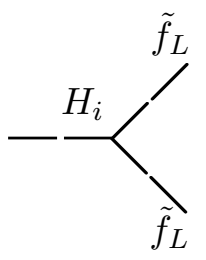

u-type

$$
\begin{aligned}
& {\left[-\frac{g m_{u}^{2}}{m_{W} \sin \beta} S_{a 2}\right.} \\
& \left.+\frac{g m_{Z}}{2 \cos \theta_{W}}\left(1-2 e_{u} \sin ^{2} \theta_{W}\right)\left(\sin \beta S_{a 2}-\cos \beta S_{a 1}\right)\right] \\
& \quad \text { d-type } \\
& {\left[-\frac{g m_{d}^{2}}{m_{W} \cos \beta} S_{a 1}\right.} \\
& \left.\quad-\frac{g m_{Z}}{2 \cos \theta_{W}}\left(1-2 e_{d} \sin ^{2} \theta_{W}\right)\left(\sin \beta S_{a 2}-\cos \beta S_{a 1}\right)\right]
\end{aligned}
$$




\section{u-type}

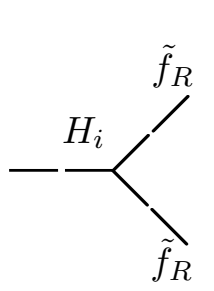

$$
\begin{aligned}
& {\left[-\frac{g m_{u}^{2}}{m_{W} \sin \beta} S_{a 2}\right.} \\
& \left.+g m_{W} e_{u} \tan \theta_{W}\left(\sin \beta S_{a 2}-\cos \beta S_{a 1}\right)\right] \\
& \text { d-type } \\
& {\left[-\frac{g m_{d}^{2}}{m_{W} \cos \beta} S_{a 1}\right.} \\
& \left.-g m_{W} e_{d} \tan \theta_{W}\left(\sin \beta S_{a 2}-\cos \beta S_{a 1}\right)\right]
\end{aligned}
$$

\section{u-type}

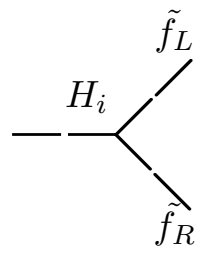

$$
\begin{gathered}
\frac{g m_{u}}{2 m_{W} \cos \beta}\left(\lambda\left(h_{u} S_{a 3}+s S_{a 1}\right)-A_{U} S_{a 2}\right) \\
\text { d-type } \\
\frac{g m_{d}}{2 m_{W} \cos \beta}\left(\lambda\left(h_{d} S_{a 3}+s S_{a 2}\right)-A_{D} S_{a 1}\right)
\end{gathered}
$$

\section{u-type}<smiles>CC(C)C(F)F</smiles>

$$
\begin{gathered}
\frac{i g m_{u}}{2 m_{W} \cos \beta}\left(\lambda\left(h_{u} P_{a 3}+s P_{a 1}\right)+A_{U} S_{a 2}\right) \\
\text { d-type } \\
\frac{i g m_{d}}{2 m_{W} \cos \beta}\left(\lambda\left(h_{d} P_{a 3}+s P_{a 2}\right)+A_{D} P_{a 1}\right)
\end{gathered}
$$




\section{right-right}

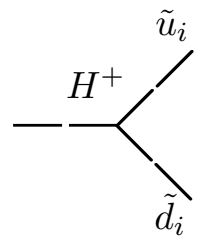

$\frac{g m_{u} m_{d}(\cot \beta+\tan \beta)}{\sqrt{2} m_{W}}$

left-left

$-\frac{g}{\sqrt{2} m_{W}}\left(m_{d}^{2} \tan \beta+m_{u}^{2} \cot \beta-m_{W}^{2} \sin 2 \beta\right)$

\section{left-right}

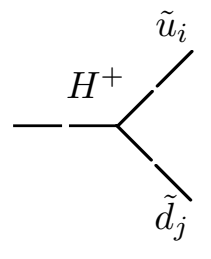

$$
\begin{gathered}
\frac{g m_{d}}{\sqrt{2} m_{W}}\left(A_{D} \tan \beta-\lambda s\right) \\
\text { right-left } \\
\frac{g m_{u}}{\sqrt{2} m_{W}}\left(A_{U} \cot \beta-\lambda s\right)
\end{gathered}
$$




\section{References}

[1] V. M. Abazov et al. Updated Combination of Searches for the Standard Model Higgs Boson at the D0 Experiment in $9.7 \mathrm{fb}^{-1}$ of Data. 2012.

[2] CDF Group, D0 Collaborations, the Tevatron New Physics, and Higgs Working. Updated Combination of CDF and D0 Searches for Standard Model Higgs Boson Production with up to $10.0 \mathrm{fb}^{-1}$ of Data. 2012.

[3] L. lvarez Gaum and J. Ellis. Eyes on a prize particle. Nature, Physics 7:2-3, 2011.

[4] T. Teubner. The standard model. In W. J Murray, editor, Proceedings of the 2010 RAL School for Experimental High Energy Physics Students, page 47. Science \& Technology Facilities Council, 2010.

[5] B. T. Cleveland, T. Daily, R. Davis, Jr., J. R. Distel, K. Lande, C. K. Lee, P. S. Wildenhain, and J. Ullman. Measurement of the Solar Electron Neutrino Flux with the Homestake Chlorine Detector. APJ, 496:505, March 1998.

[6] ALEPH, CDF, D0, DELPHI, L3, OPAL, SLD, LEP Electroweak Working Group, Tevatron Electroweak Working Group, SLD Electroweak Working Group, and Heavy Flavour Group. Precision Electroweak Measurements and Constraints on the Standard Model. 2008.

[7] U. Ellwanger, C. Hugonie, and A. M. Teixeira. The Next-to-Minimal Supersymmetric Standard Model. Phys.Rept., 496:1-77, 2010. 
[8] N. Arkani-Hamed, A.G. Cohen, E. Katz, A.E. Nelson, T. Gregoire, et al. The Minimal Moose for a Little Higgs. JHEP, 0208:021, 2002.

[9] J.F. Gunion, J. Grifols, A. Mendez, Boris Kayser, and Fredrick I. Olness. Higgs Bosons in Left-Right Symmetric Models. Phys.Rev., D40:1546, 1989.

[10] N.G. Deshpande, J.F. Gunion, B. Kayser, and F. Olness. Left-right symmetric electroweak models with triplet Higgs. Phys.Rev., D44:837-858, 1991.

[11] J.F. Gunion, C. Loomis, and K.T. Pitts. Searching for doubly charged Higgs bosons at future colliders. eConf, C960625:LTH096, 1996.

[12] N. G. Deshpande, J. F. Gunion, B. Kayser, and F Olness. Left-right-symmetric electroweak models with triplet higgs field. Phys. Rev. D, 44:837-858, Aug 1991.

[13] S.P. Das, F.F. Deppisch, O. Kittel, and J.W.F. Valle. Heavy Neutrinos and Lepton Flavour Violation in Left-Right Symmetric Models at the LHC. 2012.

[14] N. Arkani-Hamed, A.G. Cohen, and H. Georgi. (De)constructing dimensions. Phys.Rev.Lett., 86:4757-4761, 2001.

[15] H. Cheng, C.T. Hill, S. Pokorski, and J. Wang. The Standard model in the latticized bulk. Phys.Rev., D64:065007, 2001.

[16] N. Arkani-Hamed, A.G. Cohen, and H. Georgi. Electroweak symmetry breaking from dimensional deconstruction. Phys.Lett., B513:232-240, 2001.

[17] J. Lee. Neutrinos in the simplest little Higgs model. 2005.

[18] T. Han, H.E. Logan, B. Mukhopadhyaya, and R. Srikanth. Neutrino masses and lepton-number violation in the littlest Higgs scenario. Phys.Rev., D72:053007, 2005.

[19] F. del Aguila, M. Masip, and J.L. Padilla. A Little Higgs model of neutrino masses. Phys.Lett., B627:131-136, 2005.

[20] S. R. Choudhury, N. Gaur, and A. Goyal. Lepton number violation in little Higgs model. Phys.Rev., D72:097702, 2005. 
[21] A. Abada, G. Bhattacharyya, and M. Losada. Neutrinos in the simplest little Higgs scenario and TeV leptogenesis. Phys.Rev., D73:033006, 2006.

[22] A. Hektor, M. Kadastik, M. Muntel, M. Raidal, and L. Rebane. Testing neutrino masses in little Higgs models via discovery of doubly charged Higgs at LHC. Nucl.Phys., B787:198-210, 2007.

[23] J. Beringer et al. Review of Particle Physics (RPP). Phys.Rev., D86:010001, 2012.

[24] R. Barate et al. Search for the standard model Higgs boson at LEP. Phys.Lett., B565:61-75, 2003.

[25] T. Aaltonen et al. Combined search for the standard model Higgs boson decaying to a $b b$ pair using the full CDF data set. 2012.

[26] L. Lyons. Open statistical issues in Particle Physics. Ann.Appl.Stat., 2:887-915, 2008.

[27] T. Aaltonen et al. Evidence for a particle produced in association with weak bosons and decaying to a bottom-antibottom quark pair in Higgs boson searches at the Tevatron. Phys.Rev.Lett., 109:071804, 2012.

[28] V.M. Abazov et al. Search for Higgs boson production in oppositely charged dilepton and missing energy events in $p \bar{p}$ collisions at $\sqrt{s}=1.96 \mathrm{TeV}$. Phys.Rev., D86:032010, 2012.

[29] S. Chatrchyan et al. Observation of an excess of events in the search for the Standard Model Higgs boson in the $H \rightarrow Z Z^{(*)} \rightarrow \ell$ channel with the ATLAS detector. 2012.

[30] S. Chatrchyan et al. Observation of an excess of events in the search for the Standard Model Higgs boson in the gamma-gamma channel with the ATLAS detector. 2012.

[31] G. Aad et al. Combined search for the Standard Model Higgs boson in $p p$ collisions at $\sqrt{s}=7 \mathrm{TeV}$ with the ATLAS detector. Phys.Rev., D86:032003, 2012. 
[32] S. Chatrchyan et al. Observation of a new boson at a mass of $125 \mathrm{GeV}$ with the CMS experiment at the LHC. Phys.Lett.B, 2012.

[33] G. Abbiendi et al. Search for doubly charged Higgs bosons with the OPAL detector at LEP. Phys.Lett., B526:221-232, 2002.

[34] P. Achard et al. Search for doubly charged Higgs bosons at LEP. Phys.Lett., B576:18-28, 2003.

[35] J. Abdallah et al. Search for doubly charged Higgs bosons at LEP-2. Phys.Lett., B552:127-137, 2003.

[36] G. Abbiendi et al. Search for the single production of doubly charged Higgs bosons and constraints on their couplings from Bhabha scattering. Phys.Lett., B577:93-108, 2003.

[37] V.M. Abazov et al. Search for pair production of doubly-charged Higgs bosons in the $H^{++} H^{--} \rightarrow \mu^{+} \mu^{+} \mu^{-} \mu^{-}$final state at D0. Phys.Rev.Lett., 101:071803, 2008.

[38] D. Acosta et al. Search for long-lived doubly-charged Higgs bosons in $p \bar{p}$ collisions at $\sqrt{s}=1.96 \mathrm{TeV}$. Phys.Rev.Lett., 95:071801, 2005.

[39] K. Nakamura et al. Review of particle physics. J.Phys.G, G37:075021, 2010.

[40] S. Chatrchyan et al. A search for a doubly-charged Higgs boson in pp collisions at $\sqrt{s}=7 \mathrm{TeV} .2012$.

[41] G. Aad et al. Search for anomalous production of prompt like-sign muon pairs and constraints on physics beyond the Standard Model with the ATLAS detector. 2012 .

[42] S. Atag and K.O. Ozansoy. Indirect search for lepton flavor violation at CERN LEP via doubly charged bileptons. Phys.Rev., D70:053001, 2004.

[43] G.W. Bennett et al. Measurement of the negative muon anomalous magnetic moment to 0.7 ppm. Phys.Rev.Lett., 92:161802, 2004. 
[44] M. Zdrazil. Search for Doubly-charged Higgs Boson Production in the Decay $H^{++} H^{--} \rightarrow \mu^{+} \mu^{+} \mu^{-} \mu$ - with the DØDetector at $\sqrt{s}=1.96 \mathrm{TeV} . \mathrm{PhD}$ thesis, Stony Brook University, 2004.

[45] Science at Fermilab webpage, Accelerators, Fermilab's Tevatron.

[46] A. Braun, B. Evanger, C. Gattuso, P. Karns, D. Morris, R. Spayde, B. Worthel, and P. Karns. Accelerator Concepts Rookie HandBook. 2002.

[47] B.R. Martin and G. Shaw. Particle physics. Wiley, second edition, 2008.

[48] V. M. Abazov, B. Abbott, M. Abolins, B. S. Acharya, D. L. Adams, M. Adams, T. Adams, M. Agelou, J.-L. Agram, S. N. Ahmed, and et al. The upgraded DØ detector. Nuclear Instruments and Methods in Physics Research A, 565:463-537, September 2006.

[49] C. McGivern. Measurement of $W Z$ production and searches for anomalous top quark decays and Higgs boson production using tri-lepton final states in $p \bar{p}$ collisions at $\sqrt{s}=1.96 \mathrm{TeV}$. PhD thesis, University of Kanas, 2012.

[50] H. Greenlee. DØ note 4303: The D0 Kalman Track Fit. 2004.

[51] A. Khanov. DØ note 3778: HTF: histogramming method for finding tracks. The algorithm description. 2000 .

[52] A. Schwartzman and C. Tully. DØ note 4918: Primary Vertex Reconstruction by Means of Adaptive Vertex Fitting. 2005.

[53] JES Group. DØ note 5382: Jet Energy Scale Determination at D0 Run II. 2007.

[54] U. Bassler, , and G. Bernardi. DØ note 4124: Towards a Coherent Treatment of Calorimetric Energies: Missing Transverse Energy, Jets, E.M. Objects and the T42 Algorithm. 2003.

[55] O. Brandt, S. Cho, M. Cookc, M. Eads, D. Hedin, A. Santos, B. Tuchming, Y. Yatsunenko, and S. Youn. DØ note 6025: Muon Identification Certification for the Summer 2009 Extended Dataset (Run IIb-1 and -2). 2010. 
[56] P. Calfayan, T. Gadfort, G. Hesketh, V. Lesne, M. Owen, R. Stroehmer, V. Sharyy, and B. Tuchming. DØ note 5157: Muon Identification Certification for p17 data. 2007.

[57] J. Hays, J. Mitrevski, C. Schwanenberger, and T. Toole. DØ note 5105: Single Electron Efficiencies in p17 Data and Monte-Carlo Using p18.05.00 d0correct. 2006 .

[58] R. Madar and F. Couderc. DØ note 6061: Tau lepton identification using MVA optimizations, preshower and bID tools. 2010.

[59] D. Chakraborty, Y. Coadou, S. Duensing, C. Galea, Y. Gershtein, A. LeBihan, C. Noeding, and S. Protopopescu. DØ note 4210: Reconstruction of tau leptons in hadronic final states at D0 in Run 2 . 2004.

[60] G.C Blazey. In U. Baur, R. K. Ellis, and D Zeppenfeld, editors, Proceedings of the Workshop: QCD and Weak Boson Physics in Run II, page 47. FERMILABPUB-00-297, 2000.

[61] D. Chakraborty, F. Charles, C. Galea, A. Gay, Y. Gerstein, A.C Lebihan, S. Nelson, C. Noeding, C. Patwa, and S. Protopopescu. DØ note 4453: Tau ID certification for p14. 2004.

[62] T. Sjostrand, L. Lonnblad, and S. Mrenna. PYTHIA 6.2: Physics and manual. 2001.

[63] M.L. Mangano, M. Moretti, F. Piccinini, R. Pittau, and A. D. Polosa. ALPGEN, a generator for hard multiparton processes in hadronic collisions. JHEP, 0307:001, 2003.

[64] S. Protopopescu and P. Svoisky. DØ note 5094: Tau identification with neural networks for p17 data. 2006.

[65] J. Therhaag. TMVA Toolkit for multivariate data analysis in ROOT. PoS, ICHEP2010:510, 2010. 
[66] J. Kozminski, R. Kehoe, H. Weerts, S. Park, A. Quadt, and J. Gardner. DØ note 4449: Electron Likelihood in p14. 2004.

[67] A. Abdesselam. DØ note 3745: Comparison of H-Matrices for electron identification in D0 Run II. 2000.

[68] X. Bu, T. Head, K. Petridis, M. Takahashi, and Y. Xie. DØ note 6116: Electron Identification for Summer 2010. 2010.

[69] X. Bu, Y. Liu, D. Bandurin, and O. Atramentov. DØ note 5650: Artificial neural network using central preshower detector information for electron and photon selection. 2008.

[70] O. Atramentov and Y. Maravin. DØ note 4444: Utilizing CFT and SMT hits count for photon and electron reconstruction. 2004.

[71] D. Whiteson and L. Phaf. DØ note 4184: Electron Likelihood. 2003.

[72] S. Trincaz-Duvoid and P. Verdier. DØ note 4474: Missing ET Reconstruction in p17. 2004.

[73] T. Affolder et al. Charged jet evolution and the underlying event in $p \bar{p}$ collisions at $1.8 \mathrm{TeV}$. Phys.Rev., D65:092002, 2002.

[74] G. Marchesini, B.R. Webber, G. Abbiendi, I.G. Knowles, M.H. Seymour, et al. HERWIG: A Monte Carlo event generator for simulating hadron emission reactions with interfering gluons. Version 5.1 - April 1991. Comput.Phys.Commun., 67:465-508, 1992.

[75] M. Bengtsson and T. Sjostrand. Coherent Parton Showers Versus Matrix Elements: Implications of PETRA - PEP Data. Phys.Lett., B185:435, 1987.

[76] D.R. Yennie, Steven C. Frautschi, and H. Suura. The infrared divergence phenomena and high-energy processes. Annals Phys., 13:379-452, 1961.

[77] E. Boos, M. Dobbs, W. Giele, I. Hinchliffe, J. Huston, et al. Generic user process interface for event generators. 2001. 
[78] Z. Was. TAUOLA the library for tau lepton decay, and KKMC / KORALB / KORALZ /... status report. Nucl.Phys.Proc.Suppl., 98:96-102, 2001.

[79] Y. Fisyak and J. Womersley. DØ note 3191: D0gstar, D0 GEANT Simulation of the Total Apparatus Response. 1997.

[80] R. Brun, F. Bruyant, M. Maire, A.C. McPherson, and P. Zanarini. GEANT3. 1987.

[81] R. Brun and F. Rademakers. ROOT: An object oriented data analysis framework. Nucl. Instrum. Meth., A389:81-86, 1997.

[82] P. Richardson and L. Suter. Simulating the NMSSM with Herwig++. in preparation.

[83] S. Gieseke, D. Grellscheid, K. Hamilton, A. Papaefstathiou, S. Platzer, et al. Herwig ++2.5 Release Note. 2011.

[84] M. Gigg and P. Richardson. Simulation of Beyond Standard Model Physics in Herwig++. 2007.

[85] P. Skands et al. SUSY Les Houches accord: Interfacing SUSY spectrum calculators, decay packages, and event generators. JHEP, 07:036, 2004.

[86] U. Ellwanger and C. Hugonie. NMHDECAY 2.0: An Updated program for sparticle masses, Higgs masses, couplings and decay widths in the NMSSM. Comput.Phys.Commun., 175:290-303, 2006.

[87] A. Djouadi, M. Drees, U. Ellwanger, R. Godbole, C. Hugonie, et al. Benchmark scenarios for the NMSSM. JHEP, 0807:002, 2008.

[88] A. Djouadi, U. Ellwanger, and A.M. Teixeira. The Constrained next-to-minimal supersymmetric standard model. Phys.Rev.Lett., 101:101802, 2008.

[89] S. Park and M. Begel. DØ note 5324: Efficiency of the Data Quality Calorimeter Flags. 2007. 
[90] P. Calfayan. DØ note 5329: ORing single muon triggers in p17 data. 2007.

[91] J. Pumplin, D.R. Stump, J. Huston, H.L. Lai, Pavel M. Nadolsky, et al. New generation of parton distributions with uncertainties from global QCD analysis. JHEP, 0207:012, 2002.

[92] R. Hamberg, W.L. van Neerven, and T. Matsuura. A Complete calculation of the order $\alpha_{s}^{2}$ correction to the Drell-Yan K factor. Nucl.Phys., B359:343-405, 1991.

[93] J.M. Campbell and R.K. Ellis. MCFM for the Tevatron and the LHC. Nucl.Phys.Proc.Suppl., 205-206:10-15, 2010.

[94] J.M. Campbell. W / Z + B, anti-B / jets at NLO using the Monte Carlo MCFM. 2001.

[95] U. Langenfeld, S. Moch, and P. Uwer. Measuring the running top-quark mass. Phys.Rev., D80:054009, 2009.

[96] H. Schellman. DØ note 5142: The longitudinal shape of the luminous region at DØ. 2006.

[97] V.M. Abazov et al. Measurement of the shape of the boson transverse momentum distribution in $p \bar{p} \rightarrow Z / \gamma^{*} \rightarrow e^{+} e^{-}+X$ events produced at $\sqrt{s}=1.96-\mathrm{TeV}$. Phys.Rev.Lett., 100:102002, 2008.

[98] M. Shamim and T. Bolton. DØ note 5565: Generator Level Reweighting of pT of Z Boson. 2008.

[99] G. Hesketh. DØ note 5786: Generator Level Re-weighting of the Inclusive W-pT Distribution. 2008.

[100] M. Cooke and et al. $D \varnothing$ note 6031: Muon Momentum Oversmearing Update for p20 Data. 2010.

[101] O. Brandt, D. Hedin, and S. Cho. DØ note 6326: Certification of muon identification efficiencies and treatment of systematic uncertainties for the Run IIb dataset. 2012. 
[102] W. Yang. Search for MSSM Higgs Bosons in Tau Final States with the DØ Detector. PhD thesis, University of Manchester, 2010.

[103] I. Howley, L. Suter, F. Couderc, and A. Patwa. DØ note 6252: Tau ID Certification. 2011.

[104] F. Couderc and A. Patwa. Tau ID Meeting, D0 Collaboration meeting . 2009.

[105] M. Arov, C. Biscarat, D. Chakraborty, C. Galea, A. Gay, Y. Hu, Y. Gerstein, A. Le Bihan, S. Nelson, C. Noeding, A. Patwa, Y. Pogorelov, and S. Protopopescu. $D \varnothing$ note 4773: Tau identification with Neural Networks for p14 pass 2 data. 2005 .

[106] Fabrice C. $D \varnothing$ note 6195: Search for neutral higgs bosons at high tanbeta in the $\mu \tau_{h}$ final state with $7.3^{-1}$ fb of run2 data. 2011.

[107] W. Yang, S. Soldner-Rembold, M. Owen, and A. Patwa. DØ note 5708: Search for mssm higgs boson production in the decay $h \rightarrow \tau_{\mu} \tau$ with the d0 detector at $\operatorname{sqrt}(\mathrm{s})=1.96$ tev. 2008 .

[108] V.M. Abazov et al. Search for associated Higgs boson production using like charge dilepton events in $p \bar{p}$ collisions at $\sqrt{s}=1.96 \mathrm{TeV}$. Phys.Rev., D84:092002, 2011.

[109] L. Suter, K. Petridis, and S. Soldner-Rembold. DØ note 6286: Search for a standard model Higgs boson, in a $\tau \tau \mu$ final state using $\mathrm{L}=7.0 \mathrm{fb}^{-1}$ at $\sqrt{s}=1.96$ TeV. 2012.

[110] V. Buescher, J.F. Grivaz, T. Nunnemann, and M. Wobisch. DØ note 4618: Conclusions of Mini-Workshop on PDF uncertainties and related topics. 2004.

[111] G. Snow and for the Luminosity Working Group. DØ note 5139: Adjustments to the Measured Integrated Luminosity in Run IIa. 2006.

[112] F. Couderc. DØ note 6077: D0 Search for Neutral Higgs Bosons at High tanB in the final state tautaub with $4.2 \mathrm{fb}-1$ of run $2 \mathrm{~b}$ Data. 2010.

[113] W. Fisher. DØ note 5595: Collie: A Confidence Level Limit Evaluator. 2008. 
[114] V.M. Abazov et al. Search for doubly-charged Higgs boson pair production in $p \bar{p}$ collisions at $\sqrt{s}=1.96 \mathrm{TeV}$. Phys.Rev.Lett., 108:021801, 2012.

[115] M. Muhlleitner and M. Spira. A Note on doubly charged Higgs pair production at hadron colliders. Phys.Rev., D68:117701, 2003.

[116] R.V. Harlander and W.B. Kilgore. Next-to-next-to-leading order Higgs production at hadron colliders. Phys.Rev.Lett., 88:201801, 2002.

[117] Private communications with Muhlleitner, M. and Spira, M.

[118] L. Suter, K. Petridis, S. Soldner-Rembold, and Takahashi. M. DØ note 6159: Search for Doubly Charged Higgs Boson Pair Production in $\mathrm{H}^{++} \mathrm{H}^{--} \rightarrow$ $\tau^{+} \tau^{+} \tau^{-} \tau^{-}$Decays using $\mathrm{L}=6.2 \mathrm{fb}^{-1}$ at $\sqrt{s}=1.96 \mathrm{TeV} .2011$.

[119] Original Root trees from [37] provided by Kim. T and Choi.S.

[120] A.D. Martin, W.J. Stirling, R.S. Thorne, and G. Watt. Parton distributions for the LHC. Eur.Phys.J., C63:189-285, 2009.

[121] D. de Florian and M. Grazzini. Higgs production through gluon fusion: Updated cross sections at the Tevatron and the LHC. Phys.Lett., B674:291-294, 2009.

[122] C. Gerber, A. Juste, G. Otero y Garzon, and A. Tanasijczuk. DØ note 5955: Studies on the Instrumental Background in electron+jets+MET Final State Selections using p17 Monte Carlo. 2009.

[123] J. Kraus and C.L. McGivern. DØ note 6347: Search for Standard Model Higgs Boson with Trileptons and Missing Transverse Energy using Boosted Decision Trees for $9.7 \mathrm{fb}^{-1}$ of $p \bar{p}$ Collisions at $\sqrt{s}=1.96 \mathrm{TeV}$. 2012 .

[124] CDF Group, D0 Collaborations, the Tevatron New Physics, and Higgs Working. CDF note 10474: Cross Section and Branching Ratio Recommendations for Tevatron Higgs Searches. 2012.

[125] S. Alekhin, S. Alioli, R.D. Ball, Valerio Bertone, Johannes Blumlein, et al. The PDF4LHC Working Group Interim Report. 2011. 
[126] R. Benbrik, M. Gomez Bock, S. Heinemeyer, O. Stal, G. Weiglein, et al. Confronting the MSSM and the NMSSM with the Discovery of a Signal in the two Photon Channel at the LHC. 2012.

[127] F. Franke and H. Fraas. Neutralinos and Higgs bosons in the next-to-minimal supersymmetric standard model. Int.J.Mod.Phys., A12:479-534, 1997. 